\title{
Modelling Damage, Fragmentation and Progressive Collapse of Structures Using Gausian Springs Based Applied Element Method
}

\author{
Abdul Latif, Mai
}

How to cite:

Abdul Latif, Mai (2018) Modelling Damage, Fragmentation and Progressive Collapse of Structures Using Gausian Springs Based Applied Element Method. Doctoral thesis, Swansea University.

http://cronfa.swan.ac.uk/Record/cronfa48975

Use policy:

This item is brought to you by Swansea University. Any person downloading material is agreeing to abide by the terms of the repository licence: copies of full text items may be used or reproduced in any format or medium, without prior permission for personal research or study, educational or non-commercial purposes only. The copyright for any work remains with the original author unless otherwise specified. The full-text must not be sold in any format or medium without the formal permission of the copyright holder. Permission for multiple reproductions should be obtained from the original author.

Authors are personally responsible for adhering to copyright and publisher restrictions when uploading content to the repository.

Please link to the metadata record in the Swansea University repository, Cronfa (link given in the citation reference above.)

http://www.swansea.ac.uk/library/researchsupport/ris-support/ 


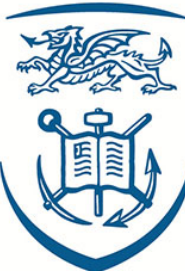

\title{
Swansea University Prifysgol Abertawe
}

\section{Modelling Damage, Fragmentation and Progressive Collapse of Structures using a Gaussian Springs based Applied Element Method}

\author{
by \\ Mai Abdul Latif \\ Submitted to the College of Engineering, \\ Zienkiewicz Centre for Computational Engineering, \\ in fulfilment of the requirements for the Degree of \\ Doctor of Philosophy \\ at \\ Swansea University
}

Adviser: Professor Yuntian Feng

September 2018 


\section{Abstract}

Modelling the progressive collapse of structures is necessary for planning controlled demolitions, studying the effect of natural disasters on structures, and determining the weakest locations of a structure for further reinforcement and enhancement. Computational mechanics served an important contribution to modelling the progressive collapse of structures, since it is very expensive to model collapse in an experimental evaluation for large scales.

Existing developments of computational methods for the scope of collapse of structures are extensively reviewed first. It is concluded that the Applied Element Method (AEM) is one of the simplest schemes for modelling the progressive collapse with sufficient accuracy. The AEM is represented as pairs of rigid elements connected by shear and normal springs, along the edges of the elements. The material properties are represented in the stiffness of the springs. The stresses and deflection between elements are based on the deflection of the springs.

The deflection and internal stresses of several structural beams are assessed using the conventional AEM and it is evident that the computational efficiency of the method is inadequate since a sizable amount of elements and springs per element is required to achieve a specific level of accuracy. Hence, a modification to the AEM is necessary to reduce the computational cost of the method. This thesis is focused on the development of the AEM for linear and nonlinear material behaviour, the development of a damage material model for representing damage and fragmentation, and an application of collapse of structures subject to earthquake and extreme wind loading.

The AEM is enhanced using the Gaussian quadrature to find the exact location of springs. Using a Gaussian distribution it is concluded that only 2 springs per element are required for elastic elements, while a total of 6 springs are required for elasto-plastic elements. In conjunction with the Gaussian springs modification, a further modification is implemented that utilises an adaptive technique for selecting the number of springs per element based on elasticity and elastoplasticity of the springs. In nonlinear material analysis the Newton-Raphson integration scheme is adapted.

To model damage in materials a softening material behaviour is employed. The developed softening algorithm is a return mapping method that is based on the predictor-corrector hardening plasticity algorithm. To represent the failure of a spring in the AEM, the stiffness of the spring is set to zero. This results in a singular global stiffness matrix that can not be solved directly. Using a dynamic model for the analysis eliminates the need of inverting the stiffness matrix, so the explicit Central Difference Method is used for linear and nonlinear dynamic analysis.

The findings in this thesis are (1) the conventional AEM is modified by chang- 
ing the distribution of the springs using the Gaussian quadrature allowing for exact calculation of optimal spring locations (2) only 2 and 6 linear and nonlinear springs are needed, respectively between a pair of elements, reducing the overall computational cost of the structure and increasing the accuracy (3) an adaptive transition springs technique is implemented and allowed for an overall reduced computational cost (4) a softening return mapping algorithm is developed for representing material damage (5) a time integrating technique is required when element separation occurs to avoid a singular matrix (6) application of the Gaussian AEM is performed on 2D frames subject to earthquake loads and extreme wind loads. 


\section{نبذة مختصرة}

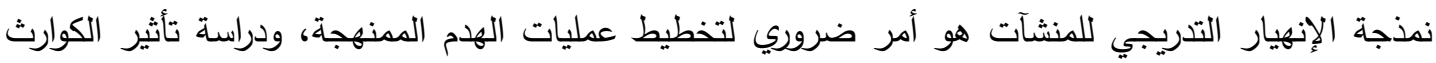

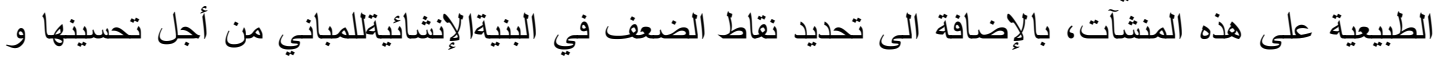

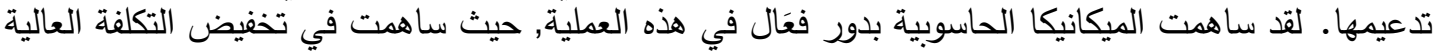

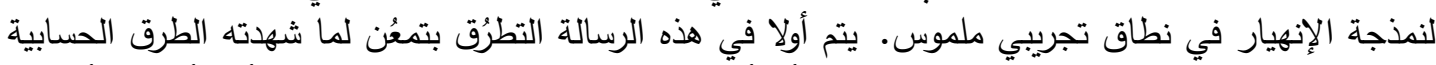
من تطورات في هذا المجال. و عليه, تم إستتتاج أن أسلوب العنصر التهات التطبيقي (AEM) هو أحد أبسط الأساليب

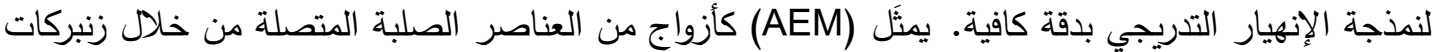

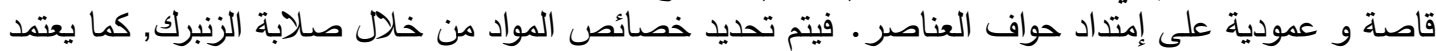

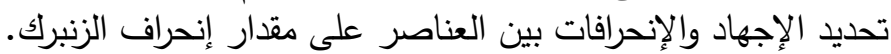

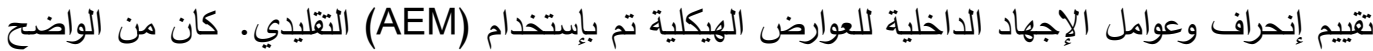
أن الفعالية الحسابية للطريقة غير كافية, حيث أن العملية تتطلب عدد كبيراً من العناصر والزئنبركات التعات المرتبطة بكل

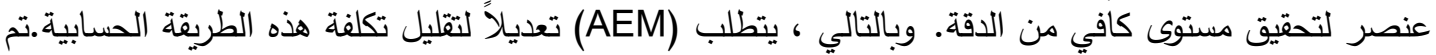

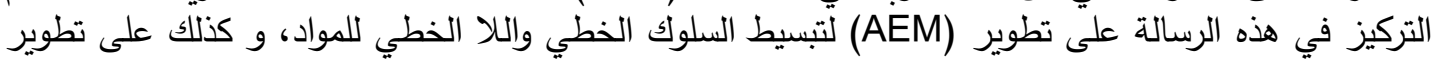

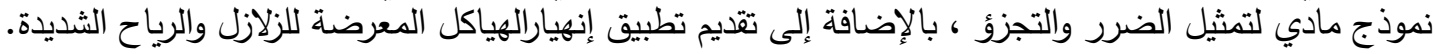

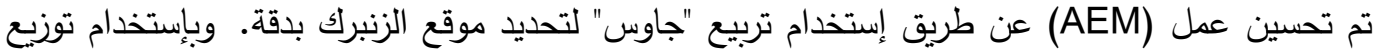

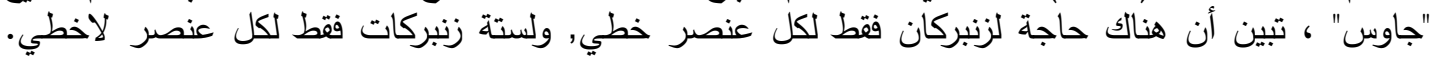

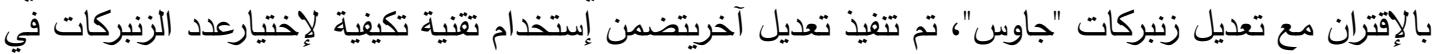

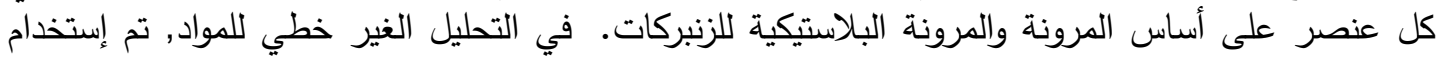
مخطط تكامل نيوتن كرافيسون.

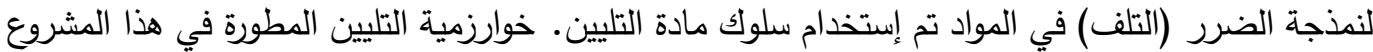

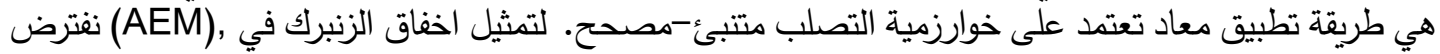

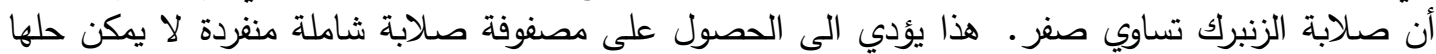

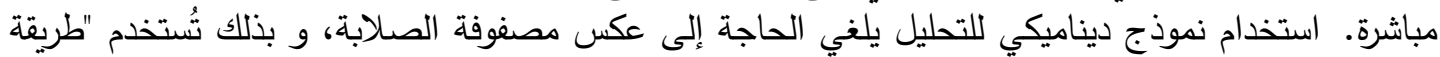

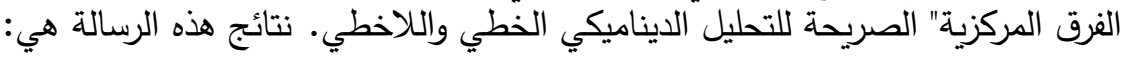

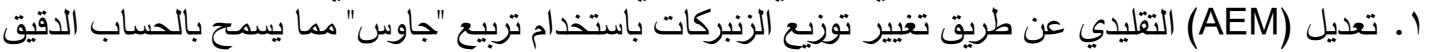
لبأماكن المثالية للزنبركات. r. الحاجة فقط لزنبركان أونبركات ستة زنبركات خطية ولا خطية على التوالي, بين زوج من العناصر ، مما يخفض من

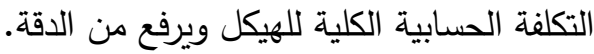
r. تتفيذ تقنية الزنبركات الانتقالية التكيفية, مما أدى الى خلى خفض التئ التكلفة الحسابية بشكل عام.

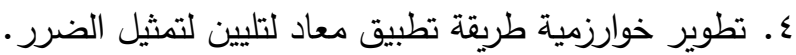

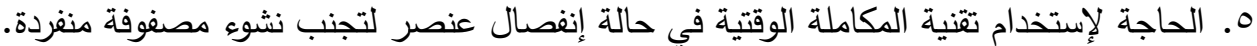
T. تم تتفيذ تطبيق (AEM) "جاوس" على إظارات ثنائية الأبعاد خاضعة لأحمال الزلازل وأعباء الرياح الثديدة. 


\section{Decleration}

This work has not previously been accepted in substance for any degree and is not being concurrently submitted in candidature for any degree.

Signed (candidate)

Date

\section{STATEMENT 1}

This thesis is the result of my own investigations, except where otherwise stated. When correction services have been used, the extent and nature of the correction is clearly marked in a footnote(s). Other sources are acknowledged by footnotes giving explicit references. A bibliography is appended.

Signed (candidate)

Date

\section{STATEMENT 2}

I hereby give consent for my thesis, if accepted, to be available for photocopying and for inter-library loan, and for the title and summary to be made available to outside organisations.

Signed (candidate)

Date 


\section{Acknowledgements}

First and foremost, I would like to express my sincerest gratitude to my supervisor, Professor Yuntian Feng, for his continuous support, his motivation, his patience and immense knowledge. He helped me realise my true potential and gave me the support I needed, especially at the moments when I doubted myself. I truly consider myself privileged for having the opportunity of working under his supervision. I could not have asked for a better mentor.

The friendships I made in the last four years will last a life time. First I have to thank, German Martinez-Ayuso and Nidhal Jemaia. You have been like family to me. I am Forever grateful for all the memories we made along the way. I thank you for the good laughs, the coffee breaks, the Crepe Vine breakfasts, and for your comfort during the stressful times. To the friends and colleagues of Swansea; Chen, Emilio, Sanjay, Anna, Katerina, Mariela, Kostis, Dimitris, Despoina, Christina, Leandro, Guillem, Mireia, Kensley, Serena, Quang, Alex L. , Alex J. and Leen. A special thanks to Siba Ewaiwi, for our phone calls over the years. My friends abroad that made it feel like I never left home, Hoda Anvar and Alia Qirreh. I feel lucky to have shared this experience with all of you and I could not have ended it sanely without you.

I would also like to thank my brother, Mohammed Nadher Abdul Latif, for being my flatmate, for sharing this experience with me, for being my best friend, and for being my home away from home. I will cherish these years we spent together, and the lifetime bond we have built.

Finally, my biggest thanks goes to my parents and sisters, Sara and Ola. I cannot begin to imagine the amount of stress I put you through in this journey. Thank you for always being there for me, for believing in me, for teaching me to dream big and for pushing me to become a better person every day. 


\section{Dedication}

To my beloved parents, Mohammed Nadher and Randa Ibrahim.

$$
\text { إلى والدي الغاليين,محمد ناضر عبد اللطيف و رنده إبراهيم }
$$

لن تكفي الكلمات للتعبير عن شكري وإمتناني لكما. إن هذا الانجاز الذي تفخرون به لم يكن ممكنا من دون دعمكم وتوجيهم المستمر لي خلال هذه السنوات، إيمانكم وثقتكم بقدرتي على تحقيق هذا، وحبكم اللا منتهي كانوا مفتاح نجاحي للوصول إلى هذه اللحظة. أهدي نجاحي لكم مع أنه بقليل أمام سعيكم الدؤب وجهودكم من أجل تكليل مسيرتي التعليمية بنجاح.

\section{بفلكم أنا أقف فخورة بهذا الإانجاز ـ شكراً}

\section{عَنْ كَثِيرِ بْنْ قَيَسِ عَنْ رَسَولِ اللَّهِ صلى الله عليه وسلم يَقُولُ:}

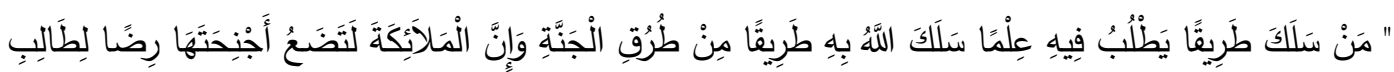

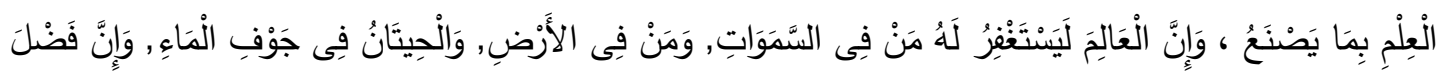

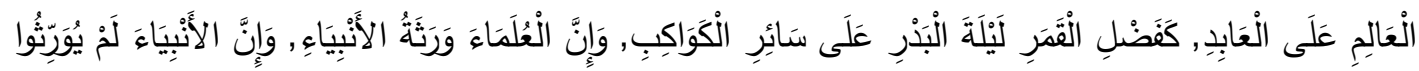

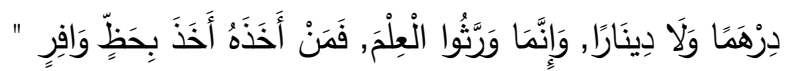




\section{Contents}

$\begin{array}{ll}\text { Abstract } & \text { i }\end{array}$

$\begin{array}{ll}\text { Arabic abstract } & \text { ii }\end{array}$

Acknowledgements vi

Acronyms $\quad$ xxv

I State of the Art of Progressive Collapse Modelling 1

1 Background 3

1.1 Motivation . . . . . . . . . . . . . . . . 3

1.2 Introduction to Progressive Collapse . . . . . . . . . . . . 5

1.2.1 Types of Progressive Collapse . . . . . . . . . . . . . 6

1.2.2 Analysis Procedure Possibilities . . . . . . . . . . . 7

1.3 Scope of the thesis . . . . . . . . . . . . . . . . 10

1.4 Outline of the thesis . . . . . . . . . . . . . . 10

2 Literature Review $\quad 13$

2.1 The Finite Element Method . . . . . . . . . . . . . . . . . . . . . 13

2.2 Discrete Element Method . . . . . . . . . . . . . . . . . . . 17

2.2.1 The Combined Finite Discrete Element Method (FDEM) 19

2.2.2 Modified Distinct Element Method . . . . . . . . . . . 20

2.2.3 Extended Distinct Element Method . . . . . . . . . . . . 22

2.3 Rigid Bodies Spring Models . . . . . . . . . . . . . . . . . . . . 24

2.4 Shifted Integration Technique . . . . . . . . . . . . . . 25

2.4.1 Adaptive Shifted Integration Technique . . . . . . . . . . 26

2.4 .2 ASI-Gauss Technique . . . . . . . . . . . . . 26

2.5 Applied Element Method . . . . . . . . . . . . . . . . . . . . . 29

2.5.1 Progressive Collapse of RC structures using ELS . . . . . 30

2.5.2 Improved Applied Element Method . . . . . . . . . . . . . . . 31

2.5.3 Voronoi Applied Element Method . . . . . . . . . . . . . . 33

2.6 Summary of Methods and Conclusion . . . . . . . . . . . . . 34

3 Numerical Methods Approaches $\quad 37$

3.1 Mechanics of Continuous Bodies . . . . . . . . . . . . . 37

3.1.1 External and Internal Virtual Work . . . . . . . . . . . . 38

3.2 Energy Principle Equations . . . . . . . . . . . . . . . . . . . . 41 
3.2.1 The Principle of Virtual Displacements . . . . . . . . . . . 41

3.2.2 The Principle of Minimum Potential Energy . . . . . . . . 42

3.3 Finite Element Method . . . . . . . . . . . . . . . . . . . . . . 43

3.3 .1 Isoparametric mapping . . . . . . . . . . . . . . . . 44

3.3.2 Jacobian of the Mapping . . . . . . . . . . . . . . . . . 46

3.3.3 Differential Volume and Area . . . . . . . . . . . . . . 49

3.3 .4 Numerical Integration . . . . . . . . . . . . . . . . . 50

3.4 Discrete Model for Continuum . . . . . . . . . . . . . . . . 51

3.5 Lattice Model for RC using EDEM . . . . . . . . . . . . . . . 54

3.6 Rigid Bodies Spring Models . . . . . . . . . . . . . 57

3.6.1 Formulation of Beam Element . . . . . . . . . . . 57

3.6.2 Plane strain elements . . . . . . . . . . . . . . . . 58

3.7 Shifted Integration Technique Formulation . . . . . . . . . . . . 60

3.7.1 Adaptively Shifted Integration Technique . . . . . . . . . 61

3.7.2 ASI-Gauss Technique . . . . . . . . . . . . . 62

\section{Formulation of Linear and Nonlinear Gaussian Based AEM}

4 Applied Element Method Formulation $\quad 67$

4.1 AEM Formulation for 2-D element . . . . . . . . . . . . . 67

4.2 AEM Meshing and Element distribution . . . . . . . . . 70

4.2.1 Structures with multiple materials . . . . . . . . . . 71

4.3 Effect of Number of Springs and Elements . . . . . . . . . . . 72

4.3.1 Effect of Number of Springs Between Elements . . . . . . 73

4.3.2 Effect of Element Size . . . . . . . . . . . . . . . . . 74

4.4 Large Displacement Analysis using AEM . . . . . . . . . . . . . . 74

4.5 Modifications in AEM . . . . . . . . . . . . . . . . 77

4.5.1 Improved Applied Element Method . . . . . . . . . . . . 77

4.5.2 Voronoi AEM . . . . . . . . . . . . . . . . 78

4.6 Conclusion . . . . . . . . . . . . . . . . . . . 79

5 Gaussian based AEM Formulation $\quad 81$

5.1 AEM for Linear Elastic Cases . . . . . . . . . . . . . . . . . . 82

5.2 Linear Elastic Case Studies: AEM vs. FEM . . . . . . . . . . . . 84

5.2 .1 Cantilever Beam . . . . . . . . . . . . . . . . 84

5.2 .2 Fixed End Beam . . . . . . . . . . . . . . . . 88

5.2.3 Simply Supported Beam . . . . . . . . . . . . . . . . . 90

5.3 Gaussian Springs based AEM . . . . . . . . . . . . . . . 92

5.3.1 Gaussian Springs Implementation in AEM . . . . . . . . . 93

5.3.2 Comparison of equal springs with Gaussian springs . . . . 94

5.4 Conclusion . . . . . . . . . . . . . . . . . . . . 97

6 Gaussian AEM in Elasto-plasticity $\quad 99$

6.1 Overview .......................... 99

6.2 Definitions . . . . . . . . . . . . . . . . . 102

6.3 Elasto-plastic Constitutive Equations Derivation . . . . . . . . . 103

6.4 Strain Hardening . . . . . . . . . . . . . . . . . . 106 
6.5 Incremental 1D rate independent plasticity . . . . . . . . . . . . . 108

6.5.1 Elastic step (Predictor Step) . . . . . . . . . . . . . . . 109

6.5.2 Plastic step (Corrector Step) . . . . . . . . . . . . . . 110

6.5.3 Return Mapping . . . . . . . . . . . . . . . . . . . . . 110

6.6 Application of AEM to elasto-plasticity . . . . . . . . . . . . 113

6.6.1 Newton Raphson Method . . . . . . . . . . . . . . . . 113

6.7 Gaussian AEM in Hardening Elasto-plasticity . . . . . . . . . . . 116

6.7.1 Cantilever beam . . . . . . . . . . . . . . 117

6.8 Adaptive Gaussian Springs . . . . . . . . . . . . . . . . . . . 123

6.8.1 Transition Point Locator . . . . . . . . . . . . . . . . 125

6.8.2 Examples . . . . . . . . . . . . . . . 126

6.9 Conclusion . . . . . . . . . . . . . . . . . 130

\section{Formulation of Damage, Fracture and Progressive} Collapse in Gaussian Based AEM \& Applications

7 Material Damage Model \& Dynamic Analysis 135

7.1 Softening Material Behaviour . . . . . . . . . . . . . . . . 135

7.1.1 Return-Mapping Algorithm verification . . . . . . . . . . . 141

7.1 .2 Failed Elements . . . . . . . . . . . . . . . . . . . . . . . . 142

7.2 Introduction to Dynamic Analysis . . . . . . . . . . . . . . . 145

7.2.1 Equation of Motion for MDOF Systems . . . . . . . . . . 145

7.2.2 Equations of Motion for Linear Systems . . . . . . . . . . 145

7.2.3 Natural Vibration Frequency and Modes Calculation . . . 147

7.3 Time-stepping Methods for linear systems . . . . . . . . . . . . . 150

7.3.1 The Central Difference Method . . . . . . . . . . . . . . . 150

7.3.2 The Newmark- $\beta$ Method . . . . . . . . . . . . . . 153

7.3.3 Numerical Methods Classifications . . . . . . . . . . . . 156

7.4 Time-stepping methods for Nonlinear Systems . . . . . . . . . . 157

7.4.1 Nonlinear Newmark- $\beta$ Method . . . . . . . . . . . . . . 157

7.4.2 Nonlinear Central Difference Method . . . . . . . . . . . . 159

7.4.3 Summary of Methods . . . . . . . . . . . . . . 160

7.5 Dynamic Formulation and Application in AEM . . . . . . . . . 161

7.5.1 Determination of Mass Matrix . . . . . . . . . . . . . . 161

7.5.2 Determination of Damping Matrix . . . . . . . . . . . 162

7.5.3 Implementing dynamics and nonlinearity in MATLAB code 163

7.6 Dynamic Analysis for Verification . . . . . . . . . . . . . . . . 164

7.6.1 Free Vibration . . . . . . . . . . . . . . . . . . . 164

7.7 Analysis Results . . . . . . . . . . . . . . . . . . 167

7.8 Conclusion . . . . . . . . . . . . . . . . . . 170

8 Structures subject to Earthquake loads 173

8.1 Introduction . . . . . . . . . . . . . . . . . . . . 173

8.1.1 How do earthquakes occur? . . . . . . . . . . . . 174

8.1 .2 Seismic Scales . . . . . . . . . . . . . . . . . 175

8.1.3 Structural Damage . . . . . . . . . . . . . . . . . 175

8.2 Earthquake Implementation in AEM . . . . . . . . . . . . . 178

8.2.1 Earthquake Data . . . . . . . . . . . . . 179 
8.2.2 Frame Implementation . . . . . . . . . . . . . . . . . 180

8.3 Applications . . . . . . . . . . . . . . . . . . . . . 180

$8.3 .11 \times 1$ Frame . . . . . . . . . . . . . . . . 180

$8.3 .23 \times 8$ Frame . . . . . . . . . . . . . . . . . 185

8.3 .3 Multi-storey frame . . . . . . . . . . . . . . . 187

8.3 .4 2x8 Frame . . . . . . . . . . . . . . . . . 188

8.3.5 High rise frame -15 floors . . . . . . . . . . . . . . . 189

8.4 Conclusion . . . . . . . . . . . . . . . . . . . 190

9 Structures Subject to Extreme Wind Loads 191

9.1 Collapse of Structures due to Wind loads . . . . . . . . . . . . . . 191

9.2 Using CFD for turbulent wind analysis . . . . . . . . . . . . . 195

9.2.1 Solution Model . . . . . . . . . . . . . . . . . . . 196

9.2 .2 CFD Model . . . . . . . . . . . . . . . . . . . . . 198

$9.2 .340 \mathrm{~m} / \mathrm{s} 3$ second gust wind . . . . . . . . . . . . . . . 201

9.2.4 $70 \mathrm{~m} / \mathrm{s} 3$ second gust wind . . . . . . . . . . . . . . 203

9.3 Loads from surrounding structures . . . . . . . . . . . . 206

9.4 Conclusion . . . . . . . . . . . . . . . . . . . . . . . 210

IV Conclusions 211

10 Concluding Remarks $\quad 213$

10.1 Conclusions . . . . . . . . . . . . . . . . . 213

10.1.1 Linear and Nonlinear Gaussian AEM . . . . . . . . . . . . 214

10.1.2 Modelling Damage Fragmentation and Progressive Collapse in $\mathrm{AEM} \ldots \ldots \ldots \ldots . \ldots . \ldots . \ldots . \ldots 216$

10.2 Concluding Remarks . . . . . . . . . . . . . . . . . . . 217

10.3 Recommendations for further research . . . . . . . . . . . 218

$\begin{array}{lll}\text { V References } & 219\end{array}$ 


\section{List of Figures}

1.1 Death rates from natural disasters $[1] \ldots \ldots 4$

1.2 Direct causes of death $[2] \ldots \ldots \ldots \ldots$

1.3 Relationship between structural damage among deceased cases [2] 4

1.4 Pancake-type Collapse $[8] \ldots \ldots \ldots 6$

1.5 Zipper-type Collapse $[8] \ldots \ldots$. . . . . . . . . . . . . . . . 7

1.6 Domino-type Collapse $[8] \ldots \ldots \ldots$. . . . . . . . . . . . 7

2.1 Element Separation Algorithms [14] . . . . . . . . . . . . . 14

2.2 Comparison of collapse mechanism using the Element Erosion algorithm, and the Node-Split Algorithm [14] . . . . . . . . . . 16

2.3 Nine storey building evolution of collapse kinematics - using NodeSplit algorithm $[14] \ldots \ldots \ldots$

2.4 Space frame discretisation using DEM [16] . . . . . . . . . . . . 17

2.5 Dome initial configuration and dimensions. Point B represents the location of the applied load $[16] \ldots \ldots \ldots \ldots$

2.6 Final configuration of Dome after load $[16] \ldots \ldots$. . . . . . 18

2.7 Collapse of structures with increasing $\alpha$ using DEM [18] . . . . . 19

2.8 Fracture process $[37] \ldots \ldots \ldots$

2.9 Fracture of a rigid-frame concrete structure under horizontal impulsive loading $[38] \ldots \ldots \ldots$. . . . . . . . . . . . . . . . 21

2.10 Fracture of masonry concrete wall with a frame subject to sinusoidal horizontal loading . . . . . . . . . . . . . . . . 22

2.11 Whole collapse of RC column using Lattice EDEM [41] . . . . . 23

2.12 Collapse of Wooden House $[48]$. . . . . . . . . . . . . . . 25 
2.13 Collapse of steel framed structure due to impact of aircraft using the ASI-Gauss technique for analysis [50] . . . . . . . . . 28

2.14 Seismic Collapse of Frame using the ASI-Gauss Technique [52] . . 29

2.15 2D Frame analysis $[55] \ldots \ldots . \ldots \ldots$

2.16 2D Frame analysis while considering debris collision [55] . . . . 30

2.17 3D Frame analysis $[55] \ldots \ldots . \ldots \ldots$

2.18 Comparison of Real Demolition vs ELS [57] . . . . . . . . . . . 32

2.19 IAEM frame collapse $[58] \ldots \ldots 33$

2.20 Comparison of crack pattern of beam [59] . . . . . . . . . . . 34

3.1 Virtual Work Done $[61]$. . . . . . . . . . . . . . . . . . . 40

3.2 Interpolation of a one-variable function . . . . . . . . . . . . 43

3.3 1D finite element $e$ with shape functions . . . . . . . . . . 44

3.4 Quadrilateral in different planes . . . . . . . . . . . . . . . . . 44

3.5 Infinitesimal quadrilateral element . . . . . . . . . . . . . 49

3.6 Discrete element method models based on rigid disc elements [64] 52

3.7 Seven-disc model . . . . . . . . . . . . . . . . . . . 54

3.8 Concrete Lattice Model $[41]$. . . . . . . . . . . . . . . . . . . 55

3.9 Beam Bending Element $[43] \ldots \ldots$. . . . . . . . . . . . 57

3.10 Plane strain element in RBSM [43] . . . . . . . . . . . . . 59

3.11 Timoshenko beam element compared to RBSM element [50] . . . 61

3.12 ASI element compared to ASI-Gauss $[50] \ldots \ldots$. . . . . . . . 63

4.12 D AEM Elements $[6] \ldots \ldots \ldots$

4.2 2D AEM Elements degrees-of-freedom [65] . . . . . . . . . . . 69

4.3 Mesh distribution for structural beam . . . . . . . . . . . . . . 70

4.4 Connectivity between adjacent elements . . . . . . . . . . . 71

4.5 Frame meshing in $\mathrm{AEM} \ldots \ldots . \ldots 71$

4.6 Representation of a cantilever concrete and steel reinforcement springs ............................... 72

4.7 Effect of Element Size $[6] \ldots \ldots$. . . . . . . . . . . . . . . . 74

4.8 Large Displacement of Simply supported beam [66] . . . . . . . . 76

4.9 Element Assembly in VAEM [59] . . . . . . . . . . . . . 78 
5.1 Noded cantilever beam diagram f . . . . . . . . . . . . 83

5.2 Local Orientation . . . . . . . . . . . . . . . . . . 83

5.3 Cantilever Free Body Diagram _. . . . . . . . . . . . . . 84

5.4 ANSYS Cantilever Deflection _. . . . . . . . . . . 86

5.5 MATLAB Cantilever Deflection . . . . . . . . . . . . . 86

5.6 MATLAB Cantilever Deflection 10 elements in x-direction opposite load . . . . . . . . . . . . . . . . . 86

5.7 MATLAB Cantilever Normal Stress . . . . . . . . . . . . . . . 87

5.8 ANSYS stress distribution . . . . . . . . . . . . . . 87

5.9 AEM stress distribution . . . . . . . . . . . . . . . . 87

5.10 ANSYS shear stress distribution . . . . . . . . . . . . . 88

5.11 AEM shear stress distribution . . . . . . . . . . . . . 88

5.12 AEM Cantilever Deflection 30 elements in x, 5 elements in y . . . 89

5.13 AEM Cantilever Deflection Load Positive Direction . . . . . . . . 89

5.14 ANSYS Fixed End Beam Deflection . . . . . . . . . . . . . . 90

5.15 AEM Fixed End Beam Deflection . . . . . . . . . . . . . . . . 90

5.16 Normal stress for FEB from ANSYS . . . . . . . . . . . . . . 91

5.17 Normal stress for FEB from AEM . . . . . . . . . . . . . . . 91

5.18 AEM Different loading direction for FEB . . . . . . . . . . . . . . 91

5.19 AEM Deflection Simply Supported Beam . . . . . . . . . . . . . . 92

5.20 AEM Normal stress Simply Supported Beam . . . . . . . . . . . . 92

5.21 Comparison of Gaussian and Equal Springs Tributary Area for 3 and 5 springs . . . . . . . . . . . . . . . . . 94

5.22 Beam with 10 elements . . . . . . . . . . . . . . . . 99 95

5.23 Fixed End Beam with 10 elements . . . . . . . . . . . . 96

6.1 Uniaxial Tension Test $[68] \ldots$. . . . . . . . . . . . . . . 100

6.2 Return mapping scheme (a) with hardening (b) no hardening [68] 111

6.3 Return Mapping with hardening . . . . . . . . . . . . . . . . . . . 112

6.4 Newton Raphson iteration - graphical representation [68] . . . . 116

6.5 Cantilever beam elements . . . . . . . . . . . . . . . . 118

6.6 Load displacement diagram at free end . . . . . . . . . . . . 118

6.7 Beam deflection through loading-unloading-loading . . . . . . . . 119 
6.8 Number of iterations required at every load step . . . . . . . . . . 120

6.9 Newton Raphson convergence . . . . . . . . . . . . . . . . . 120

6.10 Convergence at different load steps for tolerance $10^{-5} \ldots$. . . . . 121

6.11 Normal stress for cantilever beam . . . . . . . . . . . . . . . . . . 122

6.12 Shear stress for cantilever beam . . . . . . . . . . . . . . . 122

6.13 Adaptive Elastic Springs . . . . . . . . . . . . . . . . . . 123

6.14 Adaptive Elasto-plastic Springs . . . . . . . . . . . . . . . . 123

6.15 Representation of a cantilever with initial of 10 springs . . . . . . 124

6.16 Representation of a cantilever beam with all elastic springs . . . . 124

6.17 Representation of a cantilever beam using adaptive AEM springs 125

6.18 Transition Point locator . . . . . . . . . . . . . . 125

6.19 Cantilever beam with 10 elements . . . . . . . . . . . . . . 127

6.20 Deflection of cantilever beam with adaptive springs . . . . . . . . 127

6.21 Normal stress of cantilever beam with adaptive springs . . . . . . 127

6.22 Spring sets that are elasto-plastic . . . . . . . . . . . . 128

6.23 Springs distribution between elements . . . . . . . . . . . . 128

6.24 Normal stress distribution at every cross-section . . . . . . . . . . 128

6.25 Converge Rate of Newton Raphson . . . . . . . . . . . . . 128

6.26 Deflection of FEB with adaptive springs . . . . . . . . . . . 129

6.27 Normal stress distribution for Fixed End Beam . . . . . . . . . . 129

6.28 Stress with adaptive springs for FEB . . . . . . . . . . . 130

6.29 New springs distribution for FEB . . . . . . . . . . . . . . 130

6.30 New stress distribution for FEB . . . . . . . . . . . . . . 130

7.1 Strain Softening Material Behaviour . . . . . . . . . . . . 136

7.2 Trail stress predictor calculation . . . . . . . . . . . . . . 139

7.3 Demonstrating the trial function . . . . . . . . . . . . 139

7.4 Representation of $\Delta \lambda \ldots \ldots \ldots \ldots$

7.5 Plastic strain . . . . . . . . . . . . . . . . . . . . . 140

7.6 Trial stress calculation . . . . . . . . . . . . . . . . . . 141

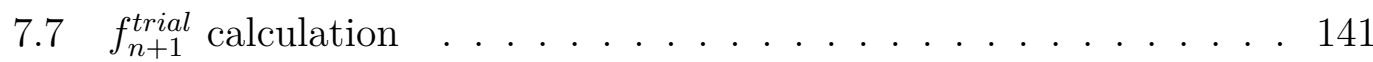

$7.8 \Delta \lambda$ and $\epsilon^{p}$ calculation . . . . . . . . . . . . . . . 142

7.9 Predictor-corrector Softening model . . . . . . . . . . . . 143 
7.10 Predictor-corrector Softening model . . . . . . . . . . . . . . . . . 144

7.11 (a) Two story shear frame (b) Forces acting on frame [81] . . . . 145

7.12 Modal Shapes for Cantilever Beam . . . . . . . . . . . . . . 149

7.13 Modal Shapes for a Fixed Ends Beam . . . . . . . . . . . . . 150

7.14 Free vibration of undamped system $[81] \ldots 165$

7.15 Free vibration of point at which load is applied . . . . . . 165

7.16 Free vibration of damped system $[81] \ldots \ldots 66$

7.17 Displacement, velocity and acceleration of damped cantilever system at free end . . . . . . . . . . . . . . 166

7.18 displacement, velocity and acceleration at free end . . . . . . 167

7.19 Beam Deflection of cantilever beam at different time steps . . . 168

7.20 Displacement, velocity and acceleration of free free end of cantilever beam for undamped system . . . . . . . . . . . . 168

7.21 Force-displacement diagram for sin load . . . . . . . . . . . 169

7.22 Displacement, velocity and acceleration at free end for cantilever beam with sin load . . . . . . . . . . . . . . . . 169

7.23 Large displacement for tall frame with no collapse . . . . . . . . . 170

8.1 Focus, epicentre and seismic waves demonstration [99] . . . . . . 174

8.2 Seismogram of Sumatra, Indonesia 2004 earthquake [101] . . . . . 175

8.3 El Centro 1940 time history acceleration graph . . . . . . . . . 179

8.4 Kobe, Japan 1995 time history acceleration graph . . . . . . . . 180

8.5 Frame with point load . . . . . . . . . . . . . . . . . . 181

8.6 Acceleration, velocity and displacement vs. time at midspan before failure occurs . . . . . . . . . . . . . . . . . . . . . . . 181

8.7 Displacement, velocity and acceleration after failure at midspan . 182

8.8 Factored frame deflection in first 0.1 second under earthquake Kobe load . . . . . . . . . . . . . . . . . . . . 183

8.9 Progressive collapse of $1 \mathrm{x} 1$ frame with earthquake Kobe loading and loading applied at midspan . . . . . . . . . . . . . . 184

8.10 Progressive collapse of $3 \times 8$ frame with earthquake loading and loading applied at midspan . . . . . . . . . . . . . 185 
8.11 Progressive collapse of 3x8 frame with earthquake loading and loading applied at midspan (continued) . . . . . . . . . . 186

8.12 Analysis of multi-storey steel frame subject to El Centro Earthquake187

8.13 Time history of a high rise frame undergoing seismic loading and point load at in positive $\mathrm{x}$-direction at first floor . . . . . . . . . 188

8.14 Time history of a high rise frame undergoing seismic loading and point load at in positive $\mathrm{x}$-direction at first floor . . . . . . . . 189

9.1 Devastating natural disasters. Red: Earthquake, Tsunami, Volcanic eruption. Blue: Flood. Green: wind-storm. Yellow: other events. [112] . . . . . . . . . . . . . . . . 192

9.2 Damage to soccer stadium in Korea due to Typhoon Rusa, 2002 $[113] \ldots \ldots \ldots \ldots \ldots \ldots \ldots$

9.3 Damage to wind energy facilities in Miyakojima island due to Typhoon Maemi, 2003 [114] . . . . . . . . . . . . . . . . . . . . 194

9.4 Broken windows in residential building in Korea, due to Typhoon Maemi [113] . . . . . . . . . . . . . . . . . . . . . 194

9.5 Collapse of building under construction undergoing intense winds [115] . . . . . . . . . . . . . . . . . 195

9.6 Flow chart for procedure . . . . . . . . . . . . 196

9.7 Configuration of the simulation for flow past a single building [116] 197

9.8 Comparison of results from FLUENT and from the wind tunnel test $[116] \ldots \ldots \ldots \ldots \ldots \ldots$

9.9 Boundary conditions . . . . . . . . . . . . . . . 199

9.10 Mesh for flow around structure . . . . . . . . . . . . . . 200

9.11 Vorticity, velocity, dynamic pressure and turbulence kinetic energy for flow past building with $40 \mathrm{~m} / \mathrm{s}$ wind speed . . . . . . . . . . 201

9.12 Residuals for turbulent flow . . . . . . . . . . . . . . . . 202

9.13 Forces on each wall in $\mathrm{x}$ and $\mathrm{y}$ directions due to $40 \mathrm{~m} / \mathrm{s}$ wind speed 202

9.14 Displacement, velocity and acceleration of a single point of the frame due to the wind load of $40 \mathrm{~m} / \mathrm{s} \ldots \ldots . . \ldots 203$

9.15 Frame displacement factored by $10^{3}$ for $3 \mathrm{sec}$ gust $40 \mathrm{~m} / \mathrm{s}$ wind speed . . . . . . . . . . . . . . . . . . 204 
9.16 Forces on left and right wall due to $70 \mathrm{~m} / \mathrm{s}$ wind speed . . . . . . 204

9.17 Contours due to the $70 \mathrm{~m} / \mathrm{s}$ wind speed, 3 second gust . . . . . . 205

9.18 Collapse of frame subject to $70 \mathrm{~m} / \mathrm{s}$ wind speed . . . . . . . 205

9.19 Geometry model for three adjacent buildings . . . . . . . . . . 206

9.20 Mesh for three adjacent buildings . . . . . . . . . . . . . . . 207

9.21 Forces on all the walls for the third building with 3 second gust

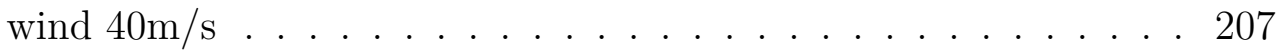

9.22 Vorticity, velocity, turbulence and dynamic pressure contours of three adjacent buildings with $40 \mathrm{~m} / \mathrm{s}$ wind speed . . . . . . . 208

9.23 Displacement velocity and acceleration for the third building at 40 m/s 3 second gust . . . . . . . . . . . . . . . . 208

9.24 Frame deflection subject to $40 \mathrm{~m} / \mathrm{s}$ wind; effect of neighbouring buildings . . . . . . . . . . . . . . . . . 209 


\section{List of Tables}

2.1 Comparison AEM vs IAEM steel beam . . . . . . . . . . . . . 32

2.2 Summary of Advantages and disadvantages of Methods . . . . . 35

2.3 Summary of methods formulation basis . . . . . . . . . . 36

5.1 Beam Properties . . . . . . . . . . . . . . . . . 84

5.2 Small Displacement comparison . . . . . . . . . . . . . . . . 86

5.3 FEB deflection Comparison . . . . . . . . . . . . . . 90

5.43 springs coordinates and weighting factor . . . . . . . . . . 94

5.53 springs $w_{i}$ and $x_{i}$ for cross-section $5 \mathrm{~m} \ldots \ldots \ldots 94$

5.65 springs coordinates and weighting factor . . . . . . . . . . 94

5.75 springs coordinates and weighting factor . . . . . . . . . 94

5.8 Deflection at free end comparison . . . . . . . . . . . . . . 95

5.9 y-deflection of beam at every node - 2 springs . . . . . . . . 96

5.10 y-deflection of beam at every node - 50 springs . . . . . . . . 96

5.11 y-deflection of beam at every node - 2 and 50 Gaussian springs . . 96

5.12 y-deflection of FEB at every node - 2 springs . . . . . . . . . . 96

5.13 y-deflection of FEB at every node - 50 springs . . . . . . . . . . . 97

6.1 Residual at each iteration . . . . . . . . . . . . . . . 120

6.2 Residual at each iteration . . . . . . . . . . . . . . . . 128

7.1 Summary of advantages and disadvantages . . . . . . . . . . . 161

8.1 Some of the Deadliest Earthquakes $[97] \ldots \ldots$. . . . . . . 174

8.2 Methods of measuring earthquakes $[102] \ldots \ldots$. . . . . . 176

8.3 Mercalli scale \& Richter scale $[102] \ldots$. . . . . . . . . . . . 177

8.4 Section Properties . . . . . . . . . . . . . . . 181 
9.1 Wind induced phenomena $[113] \ldots \ldots$. . . . . . . . . . 193

9.2 Maximum Average force applied on each wall of the frame . . . 203 


\section{Acronyms}

$\begin{array}{ll}\text { AEM } & \text { Applied Element Method } \\ \text { FEM } & \text { Finite Element Method } \\ \text { DEM } & \text { Discrete Element Method } \\ \text { EDEM } & \text { Extended Distinct Element Method } \\ \text { MDEM } & \text { Modified Distinct Element Method } \\ \text { RBSM } & \text { Rigid Bodies Spring Models } \\ \text { IAEM } & \text { Improved Applied Element Method } \\ \text { VAEM } & \text { Voronoi Applied Element Method } \\ \text { ASI } & \text { Adaptively Shifted Integration Technique } \\ \text { ELS } & \text { Extreme Loading Software } \\ \text { ACI } & \text { American Concrete Institute } \\ \text { RC } & \text { Reinforced concrete } \\ \text { DOF } & \text { Degrees of freedom } \\ \text { MDOF } & \text { Multiple degrees of freedom } \\ \text { CPU } & \text { Central Processing Unit } \\ \text { CFD } & \text { Computational Fluid Dynamics } \\ \text { CDM } & \text { Central Difference Method } \\ \text { NR } & \text { Newton Raphson } \\ \text { RANS } & \text { Reynolds averaged Navier-Stokes models } \\ \text { LES } & \text { Large Eddy Simulations } \\ \text { DL } & \text { Dead load } \\ \text { LL } & \text { Live load } \\ & \\ \text { DA }\end{array}$




\section{Nomenclature}

E Young's Modulus of Elasticity

G Shear Modulus of Elasticity

I Second moment of inertia

$K \quad$ Stiffness matrix

$K_{n} \quad$ Normal stiffness of spring in AEM

$K_{n}^{i} \quad$ Normal stiffness of spring in Improved AEM

$K_{s} \quad$ Shear stiffness of spring in AEM

$K_{s}^{i} \quad$ Shear stiffness of spring in Improved AEM

$\Delta \lambda \quad$ Consistency parameter - in hardening and softening materials

$\Delta \mathbf{u} \quad$ Incremental displacement vector

$\Delta \ddot{\mathbf{u}} \quad$ Incremental acceleration vector

$\Delta \dot{\mathbf{u}} \quad$ Incremental velocity vector

$\Delta \mathbf{f} \quad$ Incremental force vector

$\Omega \quad$ Material body

$\Pi \quad$ Potential Energy

$\alpha \quad$ Isotropic internal hardening - equivalent plastic strain

$\delta W \quad$ Virtual work

$\delta W_{E} \quad$ External virtual work

$\delta W_{I} \quad$ Internal virtual work

$\delta u \quad$ Virtual displacement

J Jacobian Matrix

$\mathbf{R}_{\mathbf{G}} \quad$ Residual force vector due to geometrical changes

$\mathbf{R}_{\mathbf{m}} \quad$ Residual force vector due to cracking or incompatibility between spring strains and stresses 
$\nu \quad$ Poisson's ratio

$\rho \quad$ Density of considered material

a Element width in AEM

$b_{n 1} \quad$ Distance between centre of element to spring location in AEM

$d \quad$ Length of spring tributary area in AEM

$d s \quad$ Surface element

$d v \quad$ Volume element

$n_{g} \quad \mathrm{~N}$ number of integration points in Gauss integration rule

$t \quad$ Element thickness in AEM

$t_{a v} \quad$ Average thickness of element 


\section{Part I}

State of the Art of Progressive Collapse Modelling 


\section{Chapter 1}

\section{Background}

\subsection{Motivation}

"Earthquakes dont kill people, buildings do"

During earthquakes, hurricanes, tornadoes or even tsunamis, structures undergo several types of structural damage that can cause total collapse in structures, and more importantly, danger to human life. Modelling the progressive collapse of structures is necessary for understanding the weakest locations of structures for further reinforcement and enhancement. Figure 1.1 shows the death toll since the 1900's to the late 2010's due to natural disasters. From the figure it is evident that the most deaths were due to extreme weather and earthquakes, with a death toll of 210,000 and 350,000 respectively.

Figure 1.2 presents the distribution of the causes of death after the Great Hanshin-Awaji Earthquake occured in 1995 [2]. The most common cause of death during this earthquake was suffocation.

The extensive study on the deaths from the Great Hanshin-Awaji Earthquake also showed that most deaths were caused by structural damage [2]. The study also showed that most people died in structures that were made of wood, rather than reinforced structures. Figure 1.3 shows the relationship of the human casualties with the structural damage and type [2]. The majority of the deaths occured due to completely collapsed structures or severly damaged buildiings, except for those that died due to burning wood buildings. 
Global death rates from natural disasters, per 100,000 (1900-2016)

Global annual death rates from natural catastrophes, measured per 100,000 people from 1900 to 2016.

The size of the bubble represents the total death rate per year.

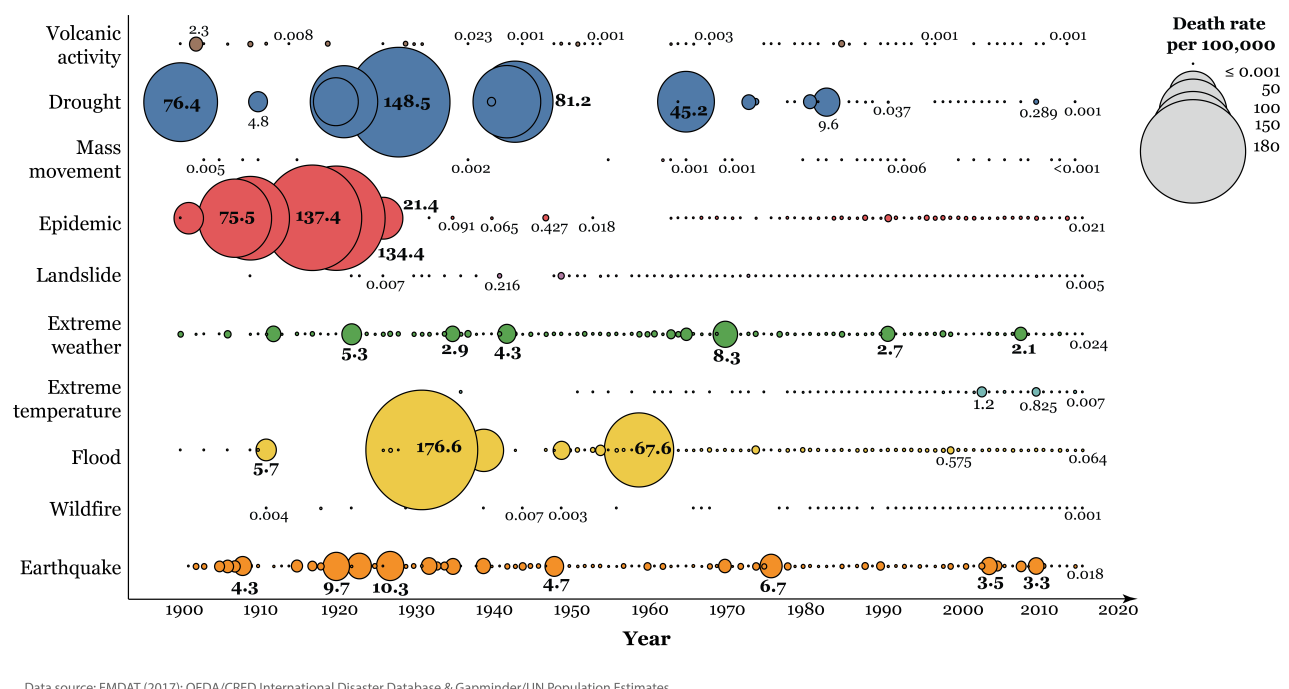

Figure 1.1: Death rates from natural disasters [1]

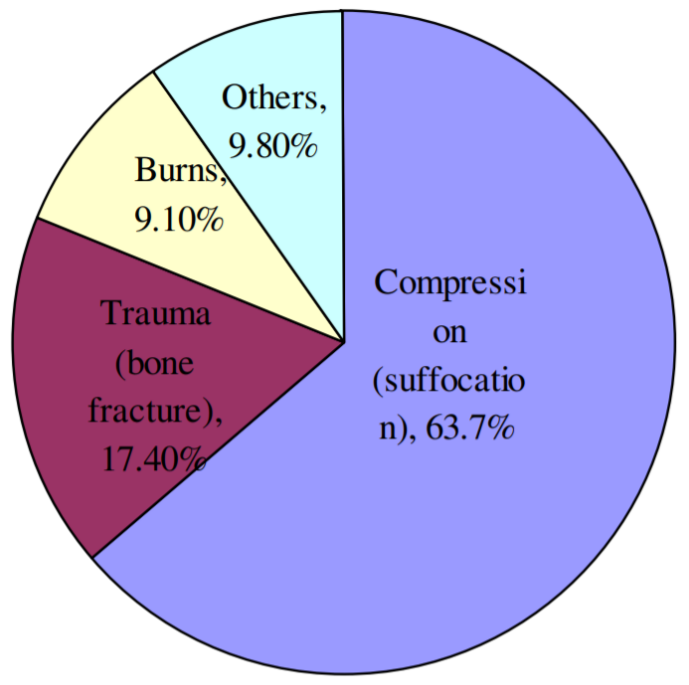

Figure 1.2: Direct causes of death [2]

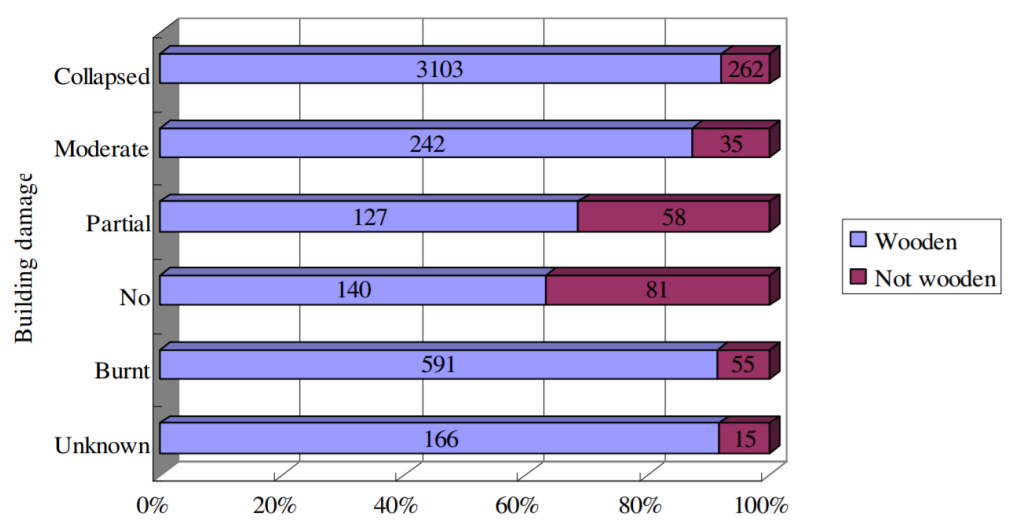

Figure 1.3: Relationship between structural damage among deceased cases [2] 


\subsection{Introduction to Progressive Collapse}

Current structural design codes posses enough collapse resistance under conventional loads. However, since structural failure is the biggest cause of death when natural disasters strike, structural engineers face a responsibility of modelling structural collapse and damage, to further aid in reinforcing buildings at their weakest points, when the structures undergo such extreme loading. The phenomena of progressive collapse has become a topic of major interest, and can be defined as follows [3];

"A progressive collapse is characterized by the loss of load-carrying capacity of a relatively small portion of a structure due to an abnormal load which, in turn, triggers a cascade of failure affecting a major portion of the structure"

In other words, once a small structural element fails, it causes a chain reaction of other structural elements to fail. Understanding the collapse state of a building can allow a higher margin of safety in design [4]. Sometimes local failures can be unavoidable if subject to very rare abnormal loading, however, understanding how to control the expansion of the failure, from local to global state that can ensure an overall stability of a structure is the important aspect.

Testing the structural collapse of buildings using experiments can be quite demanding since they require a lot of time, cost, and resources. This is why numerical methods are significant in analysing such large scale problems.

Collapse analysis requires modelling a structure from a continuous body, to a discrete one [5]. The numerical model should be able to accurately model the structure's elasto-plastic deformation and energy dissipation before collapse, as well as the rigid body movement and impact of the structural members [5].

There are several methods that can be used to analyse the behaviour of structural collapse. Currently, the available numerical models for modelling collapse are classified into two categories; continuum method (such as the Finite Element Method), and discrete element method (such as Rigid Body and Spring Model (RBSM) or the Extended Distinct Element Method (EDEM)) [6]. Nonlinear 
dynamic analysis has been widely modelled using the finite element method for analysis of progressive collapse of structures; however, difficulties in the analysis were found at the presence of excessively deformed elements with cracking or crushing, as well as having a high computational cost, and difficulties in choosing the appropriate material models for analysis [6].

\subsubsection{Types of Progressive Collapse}

Different structures are susceptible to different types of collapse and are described by a disproportion between a trigger and resulting in the collapse of a major part or the whole structure. The different types of collapse are explained in this section $[7]$.

\section{Pancake-type collapse}

The mechanism of this collapse entails an initial failure or vertical load bearing elements that result in a separation of structural components and their fall in the vertical rigid body motion. The gravitational potential energy is transformed to kinetic energy. The failure progresses in the vertical direction, and impact forces are generated. The gravitational potential energy that occurs during the fall exceeds the strain energy in the structure. Once the energy is reintroduced in the structure in an impact manner, large internal forces are generated [7]. The pancake-type collapse is better demonstrated in Figure 1.4 [8].

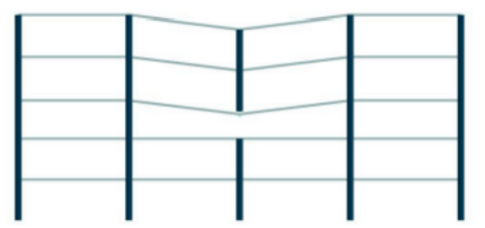

(a)

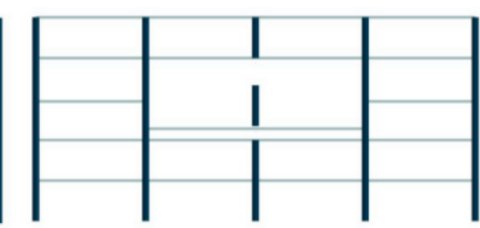

(b)

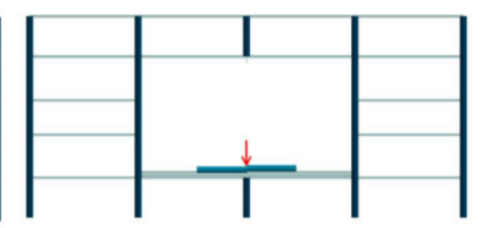

(c)

Figure 1.4: Pancake-type Collapse [8]

\section{Zipper-type collapse}

The mechanism of a zipper-type collapse entails an initial failure of one or few load bearing elements, continued by a redistribution of the forces on the elements remaining in the structure. There is a dynamic response of the 
structure to the impulsive dynamic loading due to the suddenness of the initial failure. The failure progression is in the direction transverse to the principal forces in the failing elements. The zipper-type collapse mechanism is shown in Figure 1.5 [8].

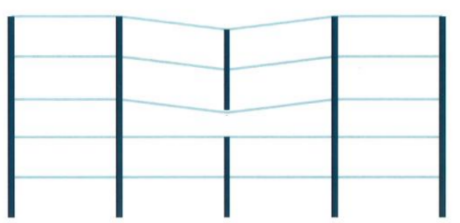

(a)

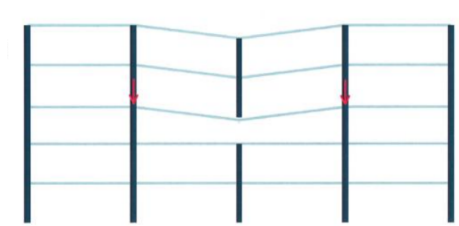

(b)

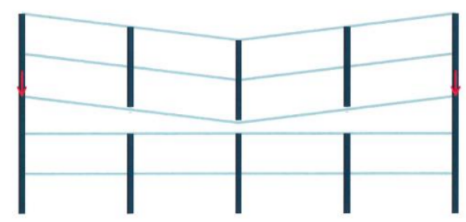

(c)

Figure 1.5: Zipper-type Collapse [8]

3. Domino-type collapse The mechanism behind a domino type collapse starts with one initial overturning of one element, that cause it to fall in an angular rigid body motion. There is then a lateral impact of the upper edge of the overturning element on the adjacent element, causing a chain reaction of the adjacent element overturning and causing an impact on its adjacent element. The mechanism is better represented in Figure 1.6 [8].

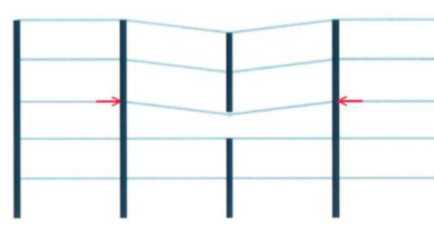

(a)

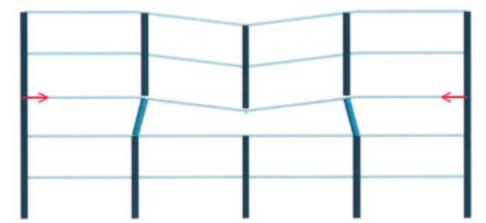

(b)

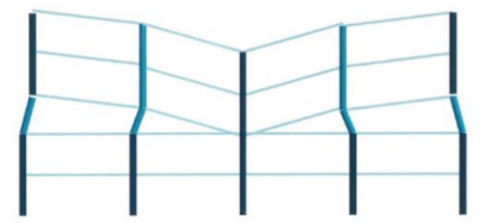

(c)

Figure 1.6: Domino-type Collapse [8]

\section{Mixed-type collapse}

The mixed type collapse includes a combination of different collapse mechanisms mentioned, as it is not necessary that only one of the mechanisms are encountered in actual collapse of structures.

\subsubsection{Analysis Procedure Possibilities}

There are several analytical methods of analysis to simulate the behaviour of structures after damage is initiated. It is important to note that the loss of 
bearing elements is modelled as a sudden dynamic removal. The most common analysis methods are [9];

1. Linear Elastic Static Analysis

2. Nonlinear Static Analysis

3. Linear Elastic Time History Analysis

4. Nonlinear Time History Analysis

The linear elastic method is considered the simplest of all the methods and the removal of of structural elements is performed statically. The method is considered relatively simple, with fast calculations. However, it does not consider dynamic effects and does not consider material nonlinear behaviour. Therefore, large or complex structures may not be evaluated with realistic results, instead it is limited to simple structures with predictable behaviour. Modelling the progressive collapse of a structure subject to a dynamic loading, such as an earthquake, will not be possible.

The nonlinear static analysis is commonly used to analyse a building undergoing a lateral load, and is known as a "push-over analysis". The applied load increases incrementally until the maximum load or displacement is reached, while undergoing nonlinear behaviour in structural members. It was found that the advantage of using this method is allowing nonlinear material behavior. However, the disadvantages outweigh the advantages since the method does not consider dynamic effects, it is expensive in terms of time, and is considered quite complex.

The linear elastic time history analysis is based on real-time removal of the major load bearing structural elements that result in real-time linear elastic motions, and can be referred to as a time history analysis. This method incorporates dynamic amplification factors, inertia and damping forces, in other words it includes dynamic behaviour. Disadvantages of the method are induced from the inability to consider material nonlinearity, time consuming, requires additional procedures for including time stepping and internal forces. The method is therefore limited to structures that may undergo large plastic deformations.

Finally, the nonlinear time history analysis provides the most realistic results from all approaches since it considers dynamic behaviour of structure, and material nonlinearity. Having those features allows larger deformations, energy 
dissipation, material yielding, cracking and failure. However, is time demanding, complex and incorrect assumptions can lead to extremely incorrect and invalid results [9]. Of all the analytical approaches it is clear that the nonlinear time history analysis is the most applicable in analysing the progressive collapse of structures.

The design approaches developed and expanded belong to one of the following categories; Performance based methods - Direct design, and Prescriptive methods - Indirect design method.

The direct design methods consider potential dangers to a structure by attempting to suitably protect the structure from the adverse affects. This approach is implemented when a threat can be identified or quantified, such as vehicle impacts, high winds, earthquakes or even flooding. In order to generate such an analysis, information about the possible forces is required, and on which elements the forces are applied [7]. There are two approaches to implement this method (a) the Alternate Path Method or (b) the Specific Local Resistance Method [10]. The alternate path method allows local failure to occur; however redistributes the forces to find an alternate load path where the damage may be absorbed to avoid a major collapse. Whereas, the local resistance method, pursues to to provide tolerable strength to resist an event [9].

The indirect design approaches are performed by removing structural elements and performing the analysis of the behaviour of the structure. For instance one approach is having a sudden loss of a column and the remaining structure is assessed. It should be noted that loss of a structural element can be a quite drastic assumption, leading to an over-design since the remainder of elements will be larger to compensate the loss. Also, every structural configuration must be considered and this cannot give a general solution [10].

The next chapter is a literature review of the numerical methods that include nonlinear behaviour of structures for progressive collapse, along with their applications. 


\subsection{Scope of the thesis}

The scope of this thesis is to improve a progressive collapse model of structures for better accuracy and efficiency, and model material damage behaviour to analyse structures subject to earthquakes and extreme wind loads. With reference to this underlying objective, this work proposes a modification to the Applied Element Method based on using the Gaussian Quadrature for improving efficiency. The AEM is coded in MATLAB. The AEM will be analysed for both linear and nonlinear material models. This thesis will cover structures in 2D only.

\subsection{Outline of the thesis}

The chapters in the thesis are organised as follows.

- Chapter 2 is a literature review of implementation of numerical methods that have been developed for modelling the progressive collapse of structures, as well as their advantages and disadvantages.

- Chapter 3 demonstrates the numerical formulations to the methods that were presented in Chapter 2. The purpose is to display the logic in the evolution of the numerical models as well as their relationships to one another, and their limitations in implementation.

- Chapter 4 is an overview of the formulation of the Applied Element method (AEM), as well as the developments to the method over the last two decades.

- Chapter 5 displays an implementation of the AEM to elastic material behaviour, with a novel modification to the springs distribution using the gaussian quadrature, called the Gaussian-AEM. Examples in how the modification improves the size of the model and accuracy are presented.

- Chapter 6 the Gaussian-AEM is implemented to nonlinear material behaviour by implementing isotropic hardening. 
- Chapter 7 the damage of materials is modelled through a softening returnmapping algorithm. A time integrating scheme is is implemented to solve problems related to fracture and damage.

- Chapter 8 the progressive collapse of structures due to earthquakes is presented for different multi-storey 2D framed structures.

- Chapter 9 the progressive collapse of structures due to extreme wind loads is presented, with an analysis of the 3 second wind gusts using CFD models.

- Chapter 10 is a conclusion of the work done in the thesis as well as future recommendations. 


\section{Chapter 2}

\section{Literature Review}

Computational models for structural analysis have been developed for almost a century. There are several methods to model continuum behaviour and discrete element behaviour. This chapter entails the different computational methods that have been developed with applicability to the progressive collapse of structures field.

\subsection{The Finite Element Method}

The finite element method (FEM) originated as early as in the 1940's. It is based on continuum material equations and has been widely applied to engineering problems including the structural analysis of large-scale structures [11]. However, some limitations in FEM arise with nonlinear and discontinuous problems [11]. Different techniques developed in the FEM for implementing dynamic behaviours with nonlinearities and discontinuities are mentioned in this section.

The finite element method typically assumes that the structures remain continuous. In some cases the collapse mechanism is represented by plastic hinges. Among other numerical methods for analysing the dynamic behaviour of continuum are: the finite difference method, the finite volume method and the boundary element method. However, these methods cannot simulate the entire failure process because additional considerations are involved where damage or fracture appears. There are some developed elasto-plastic material models for reinforced concrete FEM analysis [12, 13], but their complexity lead to large computational 
costs for calculation.

A study done by Michaloudis et al. in 2010 [14] was performed to model the structural failure of controlled demolition buildings using the finite element program LS-DYNA. The problems anticipated in modelling the collapse were due to high nonlinearities, multiple contact possibilities and the necessity for discretisation with continuum elements, which results in models with a very large number of elements. The discretisation on the LS-DYNA program was performed using 8-node solid finite elements, and a stabilisation technique developed by Belytschko-Bindeman was required. Other problems evolved from the material model. The material model did not allow detailed modification for simulating the behaviour of reinforced concrete. The explosion is modelled by removing key elements in the structure. Two different numerical models were studied: element erosion algorithm and the node-split algorithm, presented in Figure 2.1.

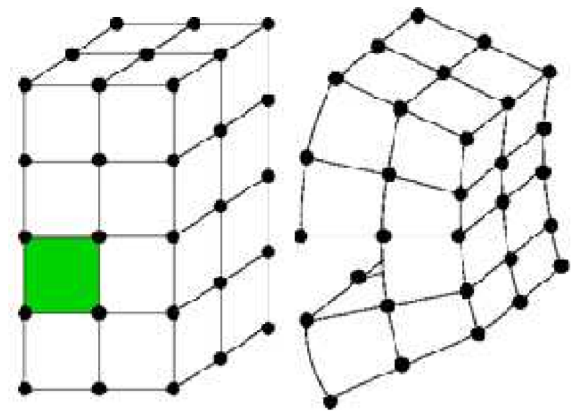

(a) Element Erosion Algorithm
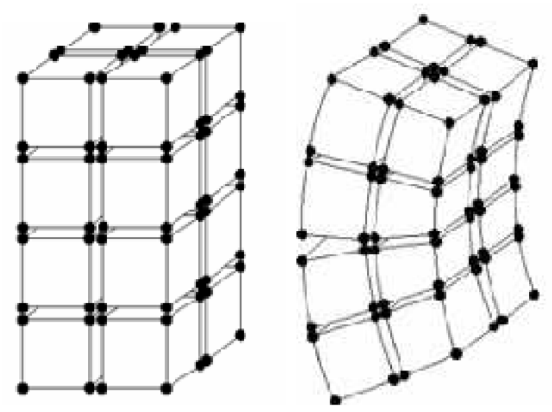

(b) Node-Split Algorithm

Figure 2.1: Element Separation Algorithms [14]

The element erosion algorithm is based on removing elements that have failed. It was noted that a 'fairly fine mesh' was required in order to capture the failure zone. This model did not allow a detailed modification to simulate the behaviour of reinforced concrete. It also deleted elements that exceed the plastic strain, resulting in a limitation in its usability, since the deletion of elements during the simulation resulted in inconsistent computations in terms of volume, mass and energy. Also, contact conditions between structural elements could not be described since contact surfaces were deleted due to the material failure. It was concluded that the results were not very useful since a very large part of the building was being deleted.

However, the results were different using the node-split algorithm. The method 
is based on that the nodes are tied (constrained) together until a failure criteria is met. Figure 2.1b shows the split behaviour when the failure criteria at a node is met. The node is split into two separate nodes and the analysis proceeds. With this method no elements are deleted, so the simulation of the structure remains consistent in terms of energy, mass and volume. Also, all contact between elements is considered. Moreover, the kinematics of the failure procedure are not affected by any kind of artificial erosion, and the contact procedures during demolition were sufficiently taken into account. Each element consists of 8 nodes, shown in Figure 2.1b. So no node belongs to more than one element at the same time, which leads to an increase in the total simulation time, and an increase in the time to construct the model, as well as an increase in the number of equations in the problem, in comparison to the element erosion algorithm.

Figure 2.2 shows a comparison analysis of the collapse kinematics and the final rest position. The figures on the left are based on the element erosion behaviour, and on the right are based on the node-split algorithm. It was concluded that both approaches provided similar behaviour of the collapse kinematics at the initial stage of the collapse and also in the whole evolution. The structures showed similar rest position, because the failure occurred mostly at the connections.

Another example of the collapse is presented in Figure 2.3, where a nine storey building undergoes two phases of blasts. Firstly, two rows of columns are removed from the fifth floor, and after 1.5 seconds two rows of columns from the second and ground floor are destroyed. The node-split algorithm is selected for the analysis, since the element erosion model will remove a very large part of the structure from the system. The results showed a realistic description of the collapse kinematics and an appropriate final rest position of the structure.

This study showed the complexities of trying to use the FEM for modelling structural collapse, even though it included several modifications to the original method. Although the method was able to present realistic final resting position of the structure as well as providing information on the possible collapse kinematics, it does not seem to be concentrating on the details of the analysis neither the accuracy. It is evident that the finite element method alone may not be the most efficient method to solve large displacement problems that include elements 

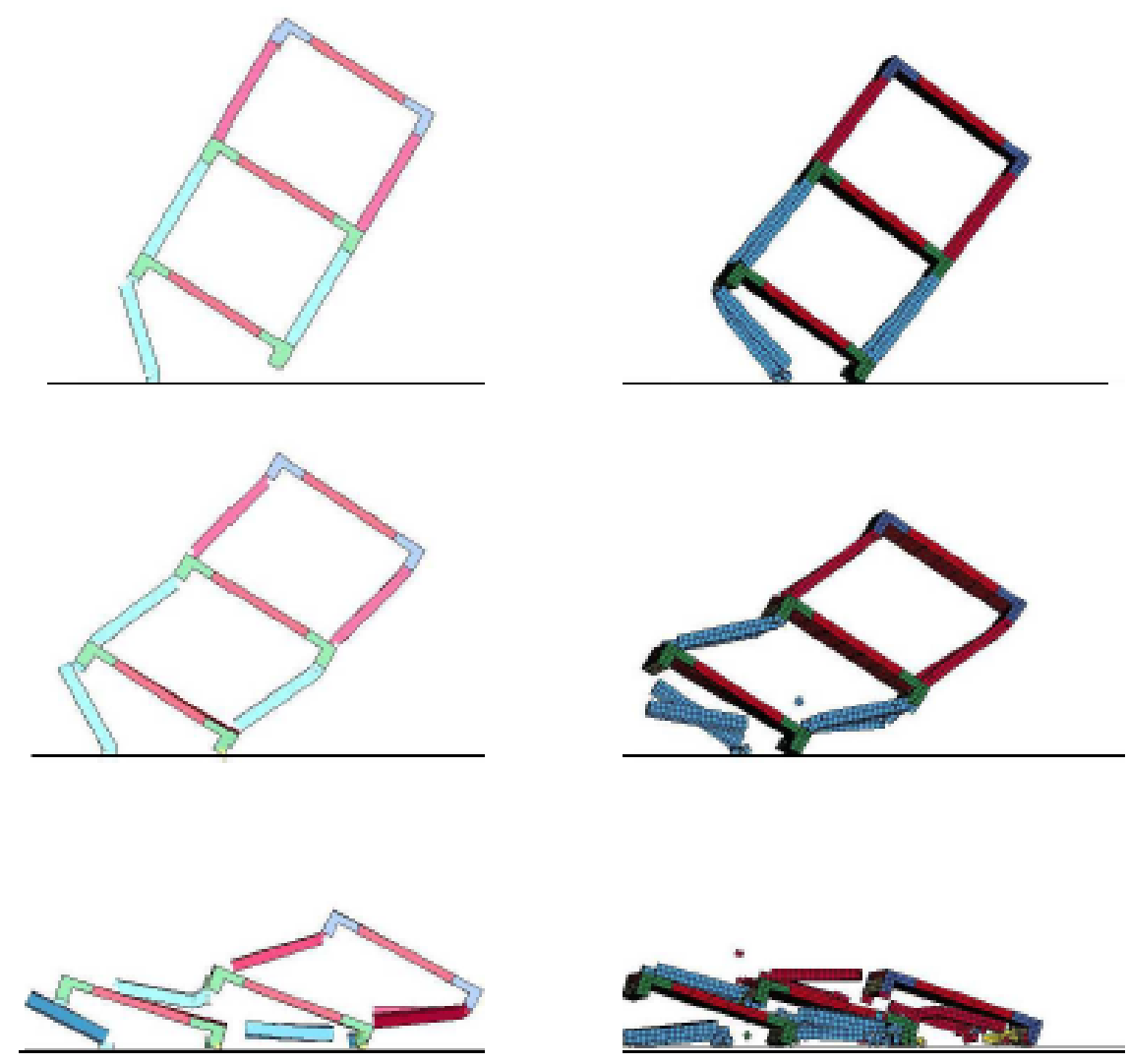

Figure 2.2: Comparison of collapse mechanism using the Element Erosion algorithm, and the Node-Split Algorithm [14]
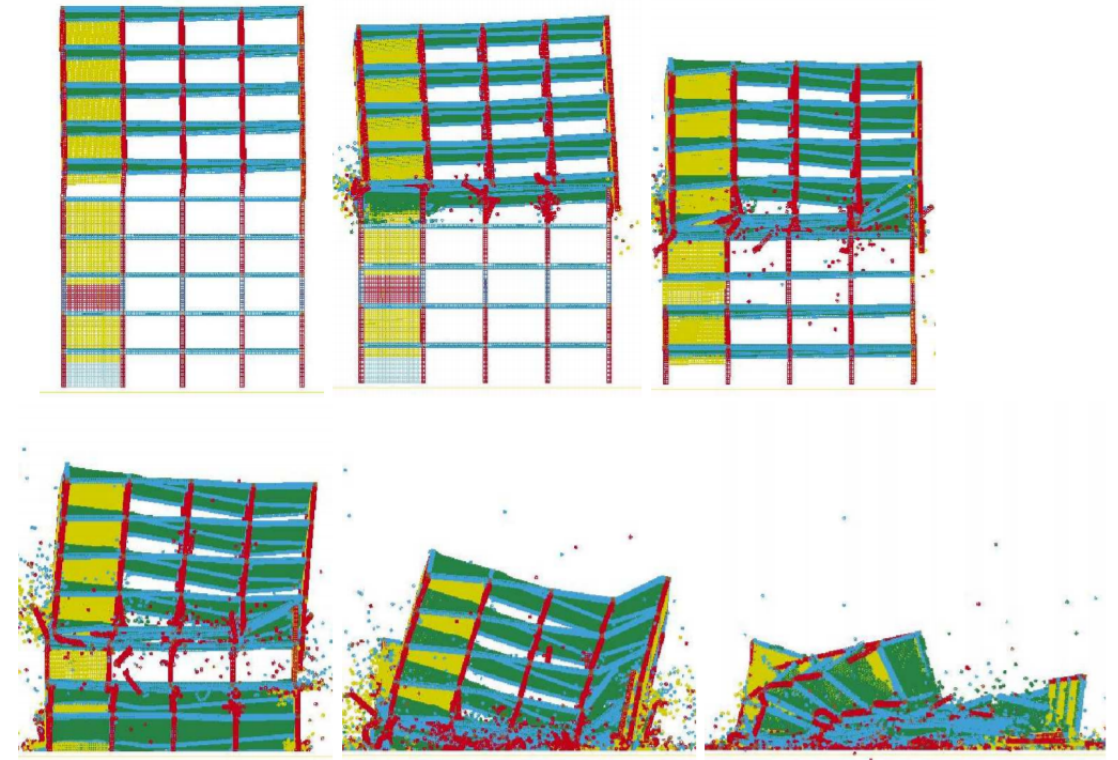

Figure 2.3: Nine storey building evolution of collapse kinematics - using NodeSplit algorithm [14] 
separation. For this reason different approaches were exploited, and are discussed in further detail in the following sections.

\subsection{Discrete Element Method}

The Discrete Element Method (DEM), also known as the distinct element method, was developed by Cundall [15], and is used to analyse non-continuum media such as rocks and granular materials. The significance of the method arises in its governing equations where no continuum condition is required, which allows it to be applicable in structural collapse. The DEM was proven very useful in geotechnical engineering since it is very effective in modelling behaviour analysis of discrete media. There have not been many applications of using the discrete method alone for progressive collapse analysis, however. The advantages of the DEM can be significant when coupled with the FEM. The coupled FEM-DEM analysis is presented in Section 2.2.1. Modifications to the DEM for better applicability in progressive collapse problems are presented in the sections 2.2.2 and 2.2.3.

\section{Large displacement using DEM}

Qi and Ye applied the DEM to nonlinear dynamic analysis of space frame structures [16]. A frame was divided into rigid spherical elements connected by spring systems between the elements to represent the discretisation using the DEM, and is shown in Figure 2.4. Using this discretisation procedure a dome under an im-
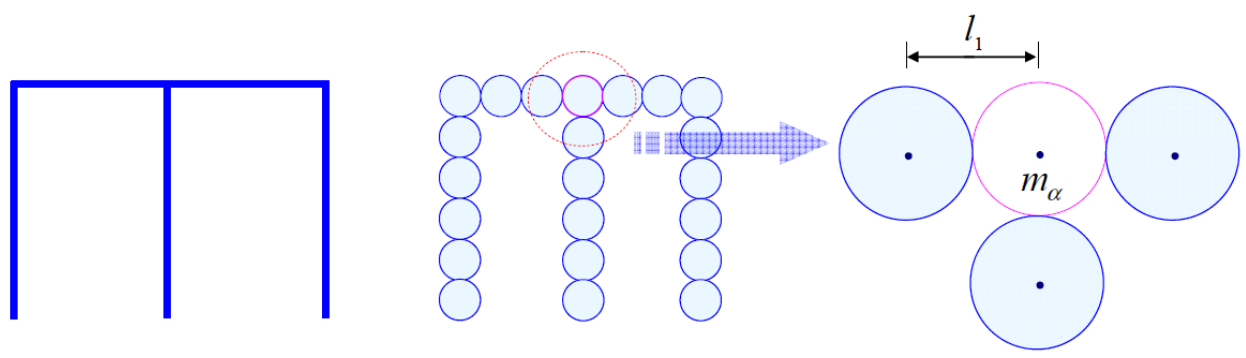

Figure 2.4: Space frame discretisation using DEM [16]

pact load was analysed. The dome geometry is shown in Figure 2.5. The dome is made of 169 nodes, and 462 members. The load is applied at point B shown in Figure 2.5. 3,355 particles were used for the DEM simulations. The deflection 
of the dome is presented in Figure 2.6. The analysis was compared to a solution from ANSYS, however no converged solution was obtained due to the large displacements the dome had undergone. Although the results were not demonstrated for progressive collapse, they showed that the DEM is applicable for use in large displacement analysis for continuum problems, and are well applied for nonlinear dynamic analysis of frame structures.

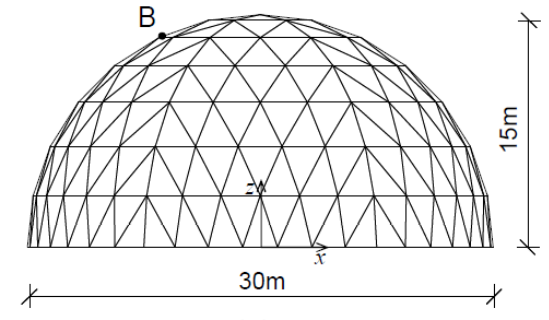

(a)

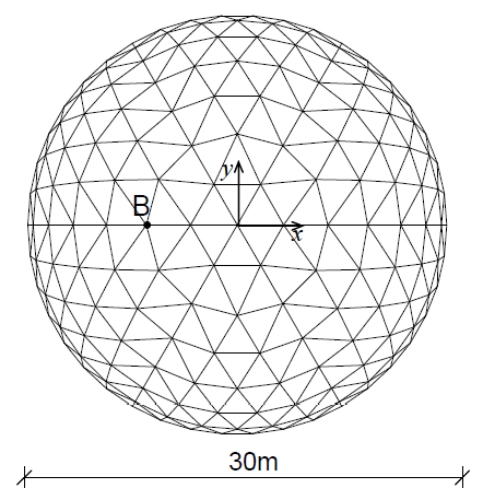

(b)

Figure 2.5: Dome initial configuration and dimensions. Point B represents the location of the applied load [16]

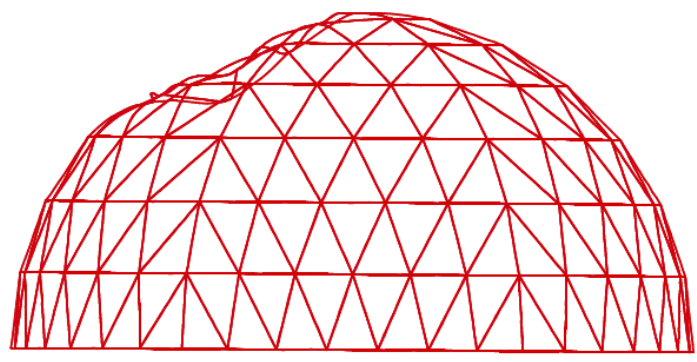

Figure 2.6: Final configuration of Dome after load [16]

\section{Collapse using DEM}

Masoero et al. showed the analysis of 3D framed structures that were subject to the removal of one column using the DEM [17]. Their analysis demonstrated that the DEM is a suitable approach for modelling the progressive collapse of structures since the mechanical response and the inter-particle contacts were captured within a robust and efficient simulation scheme [17]. They highlighted that there was a need for a fine mesh to reduce the discretisation errors of the volume representations and discrete local fracture. Also, small time increments were needed 
for the contact of stiff elements. The small time increments were due to decreases in $\alpha$, a uniform dimensionless geometry parameter. A large $\alpha$ signifies a larger element cross-sections which therefore led to stiffer and stronger structures. Figure 2.7 demonstrates the progressive collapse of the framed structures with increasing $\alpha$ over time. The time increments were in the order of $10^{-5}-10^{-6}$, and the simulation needed to run for a range of at least $2-8$ real time seconds of collapse, resulting in a very high computational cost.

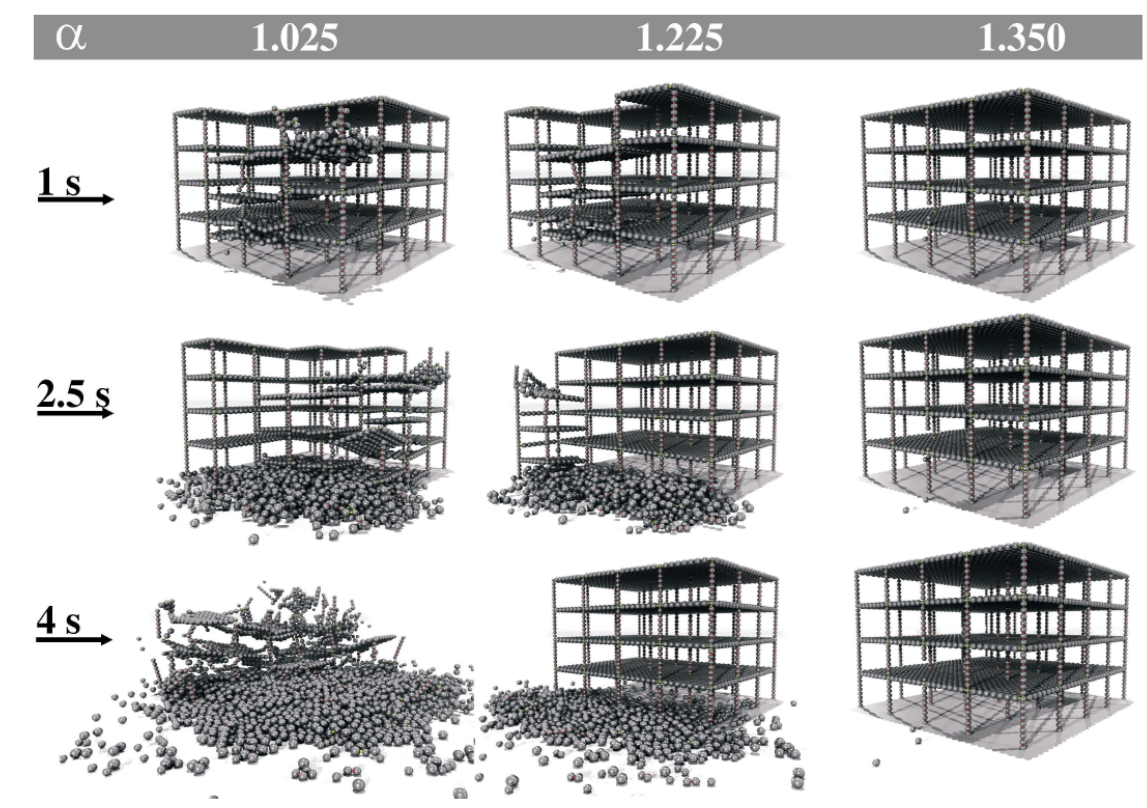

Figure 2.7: Collapse of structures with increasing $\alpha$ using DEM [18]

\subsubsection{The Combined Finite Discrete Element Method (FDEM)}

Munjiza, Owen, and Bicanic introduced a combined finite-discrete element method for modelling failing, fracture and fragmenting solids in 1990 [19]. The method is based on an incorporation of contact detection and contact interaction aspects of the discrete element method [20]- [22] into the finite element method. The contact interaction requires an accurate representation of the geometry of the contacting domains. This is achieved through discretised contact solutions. The discretisation of the contact and the deformation is assumed to coincide with the finite element discretisation in order two avoid having two separate discretisations [23]-[28]. The discretised contact solutions are used for both contact detection 
and contact interaction [28] - [31]. The combined fem-dem has been implemented in many applications and enables simulation of initiation and propagation of a large number of cracks in both 2D and 3D solids [32] -[37].

In [37], a finite strain-finite displacement shell model was developed and incorporated to the combined FEM-DEM. A thin glass shell was analysed using the combined finite-discrete element method, shown in Figure 2.8. The shell has a 50 $\mathrm{mm}$ radius, and $1 \mathrm{~mm}$ thickness, and is subject to an impact of a $50 \mathrm{~g}$ impactor at a speed of $5 \mathrm{~m} / \mathrm{s}$. The radial cracks are clearly captured. The large blocks of the shell move while the small fragments are being pushed by the impactor. The method is suitable for analysis of progressive fracture and fragmentation of shell structures with large number of cracks.

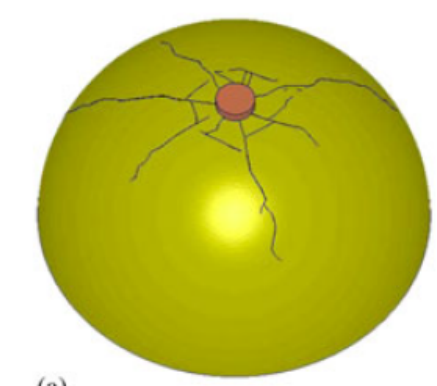

(a)

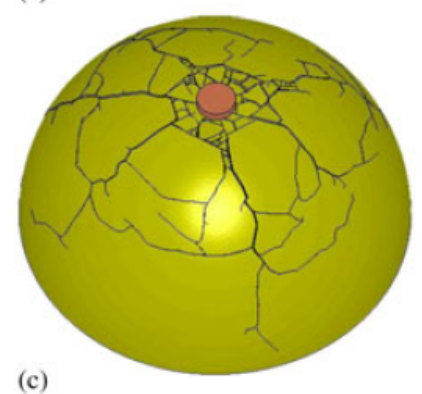

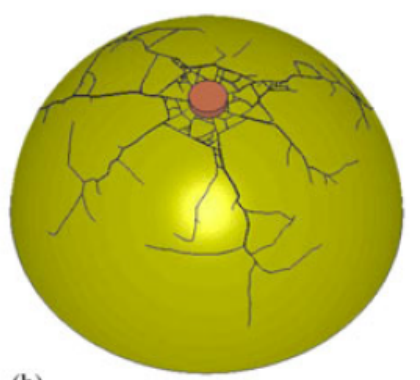

(b)

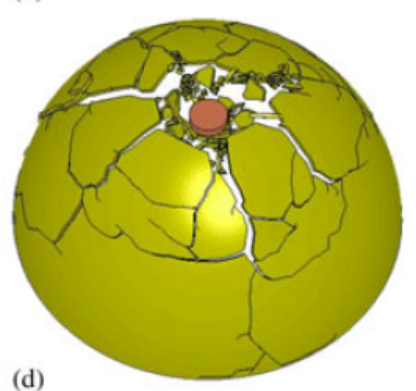

(d)

Figure 2.8: Fracture process [37]

\subsubsection{Modified Distinct Element Method}

Meguro and Hakuno extended the distinct element method to account for fracture of concrete structures [38], calling it the Modified Distinct Element Method (MDEM). The method has been applied to problems of concrete fracture, which could not be solved by the conventional DEM [38]. In MDEM, the concrete gravel and mortar are represented as circular particle elements and nonlinear springs referred as pore-springs. The model can therefore include continuity because of the 
pore-spring. The model gradually becomes plastic as the pore-springs are fractured. The MDEM automatically conveys the nonlinearity of the medium. While the pore-springs are unyielding, the medium behaves as a continuum. Once the pore-springs are destroyed then the model starts to behave as a discrete body. Therefore, MDEM can follow the total fracture process, including the discontinuous medium [38]. The MDEM was used to simulate the dynamic fracture behaviour of concrete structures. Figure 2.9 shows the analysis of a frame that is subject to a horizontal impulsive load. The three diagrams in the first row of the figure show the normal compressive force distributions. The left diagram on the top row shows the particle distribution in the initial state. While the diagrams in the bottom row are the mortar spring distributions [38].

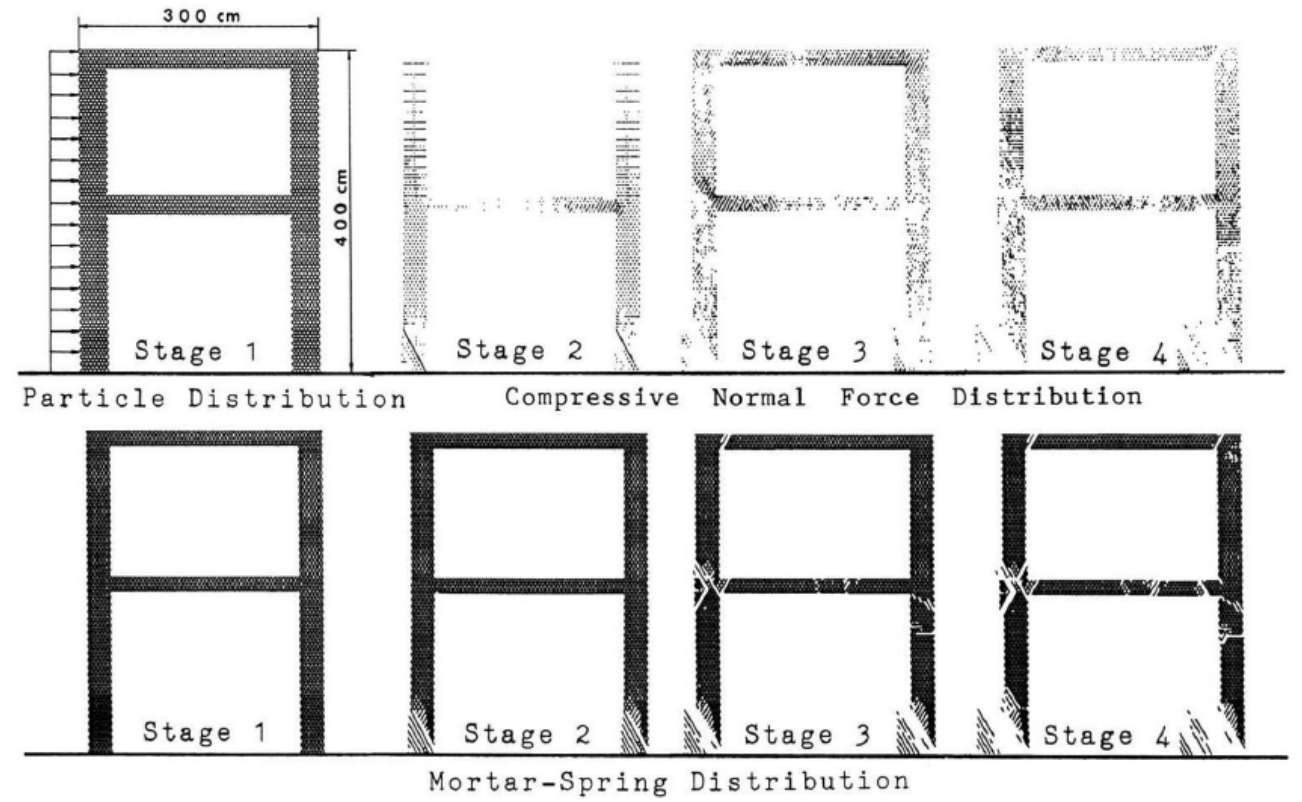

Figure 2.9: Fracture of a rigid-frame concrete structure under horizontal impulsive loading [38]

Figure 2.10 shows the fracture mechanism of the masonry concrete wall subject to horizontal sinusoidal loading. As presented, the fracture occurs within the wall. Cracks appear at stage between the column and the bottom wall. The findings of using the method on different structures with different dynamic loadings showed good agreement with laboratory tests performed on specimens in the past as well as comparison with actual seismic damage that was observed from past earthquakes [38]. 


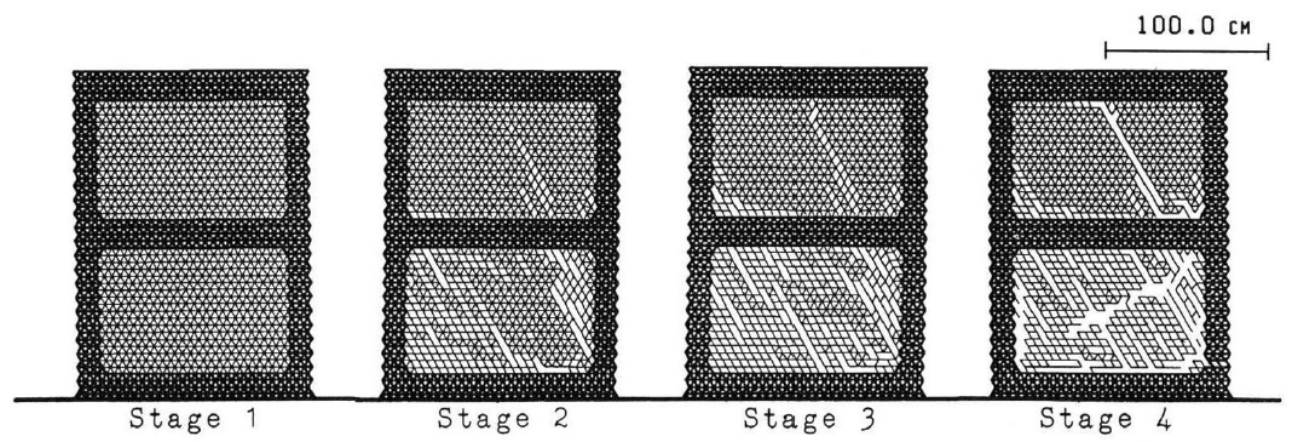

Figure 2.10: Fracture of masonry concrete wall with a frame subject to sinusoidal horizontal loading

\subsubsection{Extended Distinct Element Method}

Another modification to the original DEM is presented in this section done by Hakuno and Meguro [39][40], and is called the Extended Distinct Element Method (EDEM). The EDEM was developed where pore springs between rigid bodies could deal with continuous media. However the results from the papers explained that the EDEM requires a large calculation time because it involves explicit numerical integration, which requires a very small time step. Therefore the method is computationally very expensive for application to structures [39][40].

Sun et al. proposed a lattice element model for collapse analysis of RC bridges subject to earthquakes by using the EDEM [41]. The lattice method was developed in Reference [42], and it was compared to the conventional FEM. It was found that the analysis required less nodes, and a shorter CPU time [41]. Sun et al. proposed a model similar to the lattice element model in Reference [42], but the difference is in the details of modelling. With the model, the entire process of a structural response to an earthquake, including partial failure and collapse of whole structures was successfully simulated with good accuracy and CPU time [41].

The lattice model is composed of two parts, pre-fracture and post-fracture of the connecting springs. A concrete element consists of lumped masses connected to one another by truss elements (concrete springs), and the reinforcement bars are modelled as a discrete model, and an integrated model is used to describe the main steel bars. The constitutive relations of the springs are presented by onedimensional material models. After spring fracture, the dynamic characteristics 
are described by DEM. The fracture of the concrete and steel are determined by the ultimate strain of the material. A re-contact spring model is also proposed to simulate re-contact between the concrete masses after springs are fractures. The structure gradually changes from elastic to plastic and then to a discontinuous medium as the springs yield. Also, the effect of structural damping is mimicked by a viscous damper that is connected with a truss spring. The damping is assumed as a Rayleigh damping in proportion to the connected mass and spring stiffness. Experimental results of a RC cantilever column subjected to a static horizontal cyclic load was used to verify the lattice model developed, and only in the precollapse analysis. The results of this analysis showed acceptable results, for the pre-collapse stage. The use of EDEM with the lattice model allowed to analyse the process from elastic to collapse [41]. It was noted that the results were acceptable but further work is required to improve the accuracy of the simulations before collapse. Also, benchmark simulations must be compared with other existing models to further verify the method.

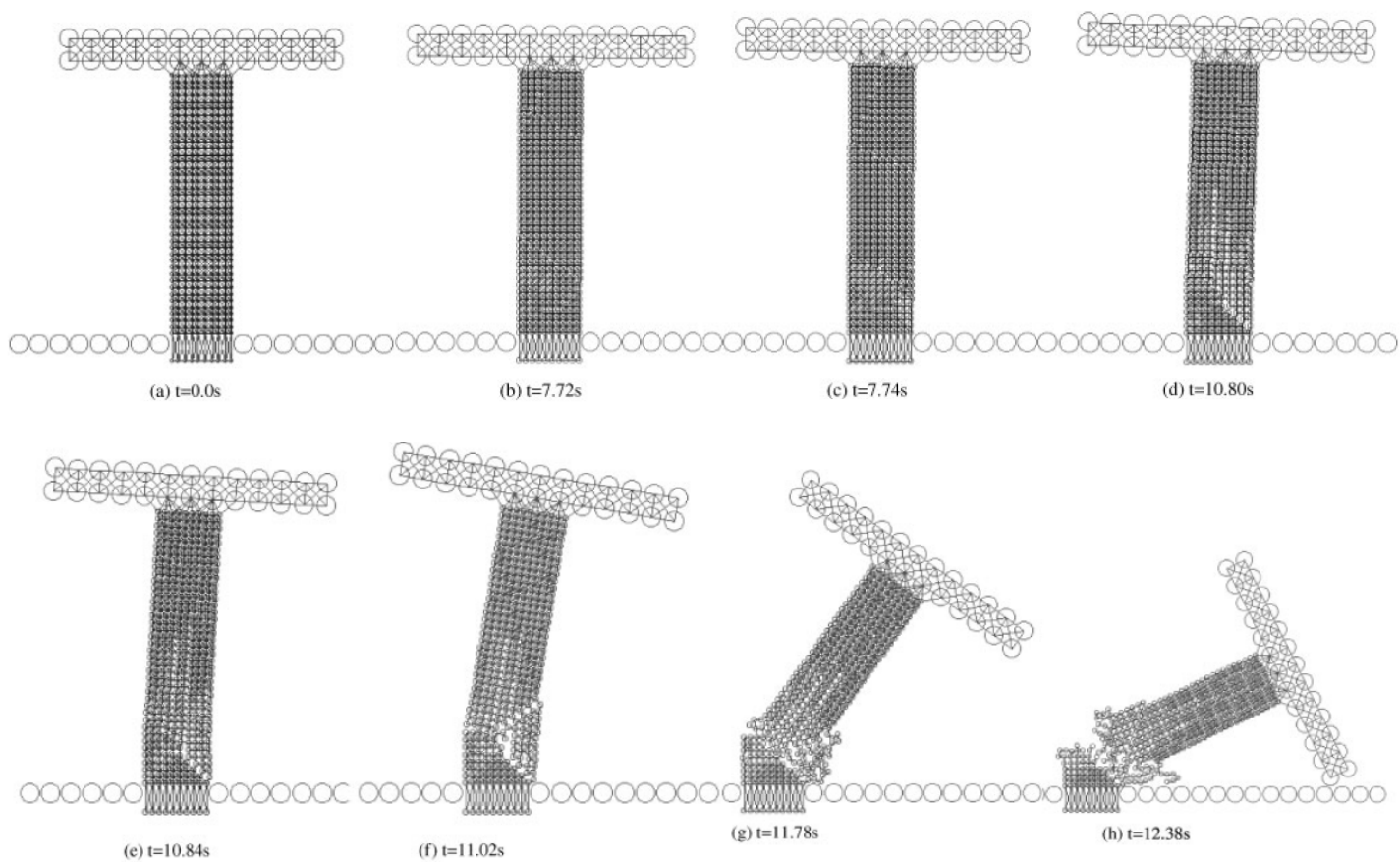

Figure 2.11: Whole collapse of RC column using Lattice EDEM [41] 


\subsection{Rigid Bodies Spring Models}

The Rigid Bodies-Springs Model (RBSM) was developed by Kawai in 1977, as a method that can analyse structures undergoing cracking or sliding. RBSM are discrete models that are composed of rigid elements connected with two types of springs, one resisting dilatation, and the other resisting shearing. They are suitable for the plastic collapse analysis of structures using the concepts of plastic hinges, hinge lines and slip lines introduced by zero spring constraints after yielding [44]. The accuracy and convergence of the linear elastic solutions are not guaranteed [45]. The effects of the finite rotation of the blocks on the collapse load and mechanism were not taken into effect [46]. Accuracy and convergence of linear elastic solutions are not guaranteed because normal strains and stresses in the direction of inter-element boundaries are neglected, except in the case of one-dimensional elements [47].

A. Tingatinga, Kawakami, and M. Shrestha presented a study to model a three dimensional collapse simulation of wooden structures using the RBSM under an earthquake load. The approach of RBSM is to divide the structure into appropriate number of rigid elements connected by spring systems [48]. A nonlinear analysis was carried out by using nonlinear springs to model the large displacements [48].

A. Tingatinga, Kawakami, and M. Shrestha analysed the collapse of typical wooden houses in Japan that are subjected to a doubly amplified 1995 Kobe earthquake. The collapse of this structure is shown in Figure 2.12. A link system used to model the plastic hinges were able to simulate the local failure causing the total collapse of the house. Also, using this method, the weak points of the structure became easily identifiable and reinforcement plans were presented by the authors of this analysis [48]. 


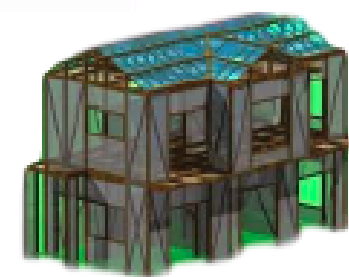

$0.00 \mathrm{~s}$

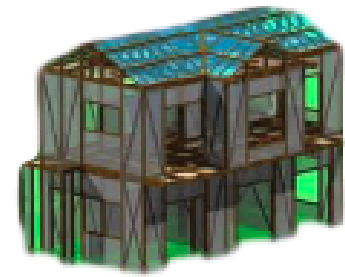

$1.50 \mathrm{~s}$

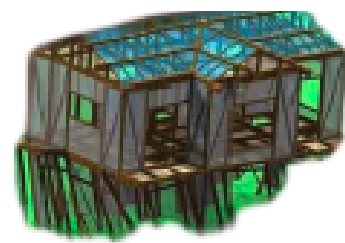

$3.00 \mathrm{~s}$

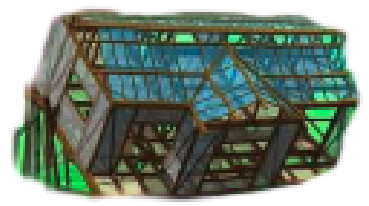

$3.75 \mathrm{~s}$

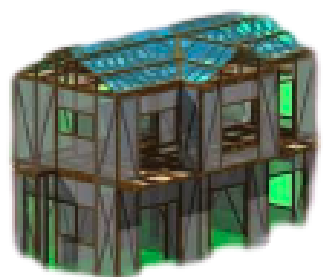

0.50

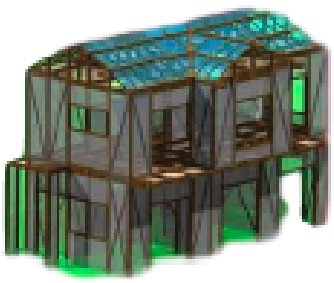

$2.00 \mathrm{~s}$

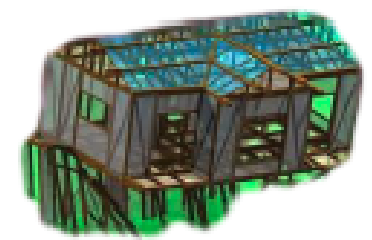

$3.25 \mathrm{~s}$

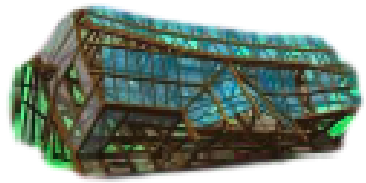

$4.00 \mathrm{~s}$

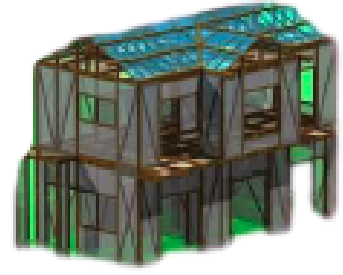

$1.00 \mathrm{~s}$

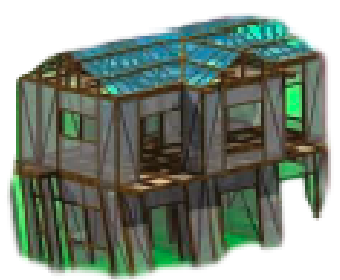

$2.50 \mathrm{~s}$

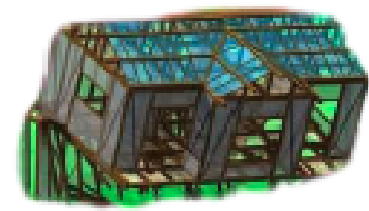

$3.50 \mathrm{~s}$

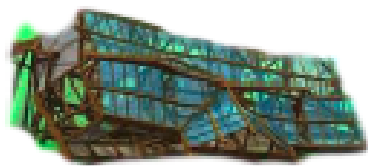

$4.25 \mathrm{~s}$

Figure 2.12: Collapse of Wooden House [48]

\subsection{Shifted Integration Technique}

Toi developed the shifted integration technique for modelling collapse of structures using the finite element method by using linear and cubic finite elements for beams and axisymmetric shells. The strain energy approximations were compared to the RBSM [47]. Toi was the first to find the relations between the locations of numerical integration points and the points with occurrences of plastic hinges. 
The linear Timoshenko beam element and the cubic beam element, as well as the RBSM, can be used in plastic collapse analysis by using plastic hinges, where the occurrence of plastic hinges can be controlled by the shifting of locations of the numerical integration points. This shifting technique, defined as the 'shifted integration technique', was shown to be effective in the finite element collapse analysis of framed structure. The shifting formed plastic hinges at the exact locations where they were expected to form, such as at clamped ends, member joints and concentrated load points locations. There was a minor loss in the accuracy of displacement, but the accuracy of the solutions for plastic collapse load had improved. The equivalence conditions between the strain energy approximations of those finite elements and the physical models, the rigid-bodies-spring-models, in which the locations of stress evaluations and plastic hinge formulations are explicitly given, were considered [47].

\subsubsection{Adaptive Shifted Integration Technique}

Toi and Isobe implemented an adaptation procedure to improve the accuracy of the displacement solution [49]. The numerical integration points in an an elastically deformed element are placed at the optimal points for linear analysis, which are the midpoint in the linear Timoshenko beam element, and Gaussian integration points in the cubic beam element. The shifted integration technique is applied immediately after the occurrence of a fully plastic section to form a plastic hinge exactly at that section. This procedure is called the Adaptively Shifted Integration Technique (ASI) [49]. It was found that this method gave accurate results in plastic collapse analysis with low number of elements. Since a low number of elements was attainable, the computational cost of a large-scale plastic collapse analysis is drastically lowered. It was noted that buckling and dynamic collapse problems were to be considered in future analysis.

\subsubsection{ASI-Gauss Technique}

Further research has been done by Lynn and Isobe to improve the ASI into a modified ASI-Gauss technique, where numerical integration points are placed in a way that the stresses and strains are are calculated at the Gaussian integra- 
tion points of the two element member. The results showed that the ASI-Gauss technique had higher accuracy than the ASI technique in the elastic range [11]. Lynn and Isobe performed an elasto-plastic analysis under static and dynamic loading, and it was confirmed that "nearly confirmed" solutions were obtained when the number of elements per member is two. Furthermore, due to the difficulty in determining the loads that result from impact in structures, applying the impact loads as nodal forces deems to falsely simulate impact[11]. The impact phenomenon was presented by means of contact between elements involved, using a contact algorithm.

Lynn and Isobe also applied the ASI-Gauss technique a dynamic analysis with high non-linearities and discontinuities, by considering the impact of an aircraft against a 10 storey steel framed structure. The structure was assumed to be made of steel, while the aircraft was made of duralumin. The 10 storied structure was assumed to be fixed at the lower ends. The aircraft had zero degree of inclination from the horizon, and an initial velocity was applied to it [11]. The collapse of the structure due to the impact is shown in Figure 2.13. The results showed a relatively low computational cost with reference to the scale of the problem. The authors mentioned that further study is required to clarify the effect of the size of the aircraft on the impact damage and to evaluate the structural damage caused by the tensile stresses of the columns [11]. The only difference between ASI and ASI-Gauss is in the initial location of the numerical integration point [50].

Katahira et al. implemented the ASI-Gauss technique into a nonlinear finite element code to develop an accurate seismic collapse simulator, including considering structural discontinuities [51]. A comparison of the conventional finite element scheme in which the numerical integration point of each element is fixed at the midpoint, the ASI technique and the ASI-Gauss technique was also presented. Analysis of a frame under seismic excitation was performed. The results showed that the conventional finite element scheme had a very slow convergence and only converges when a 64-element model is used. The ASI technique showed faster convergence results than the finite element scheme, but not with two element model since there is deficiency in accuracy in the elastic stage. The ASI-Gauss technique showed a very fast convergence, since the stress evaluation points are 


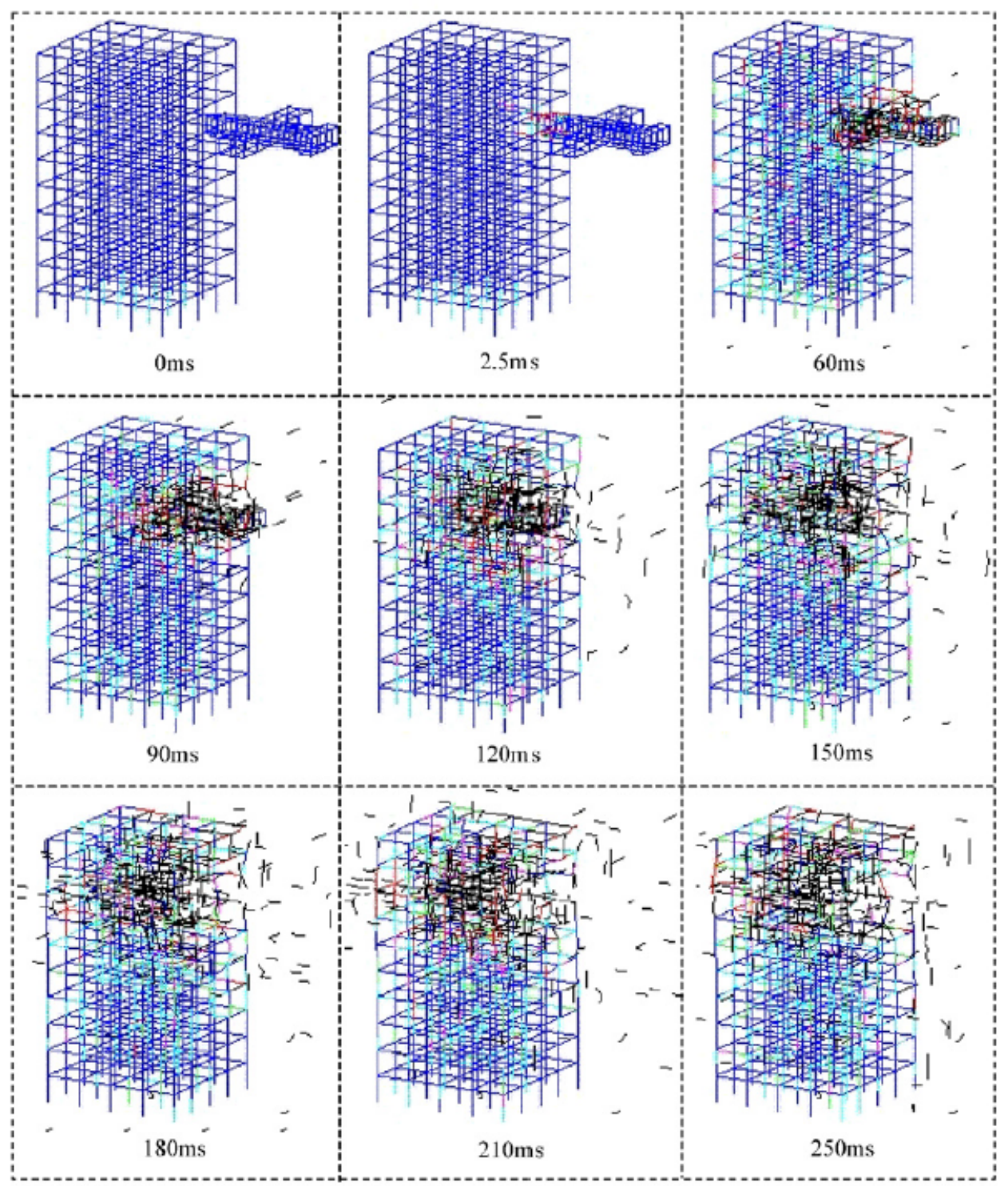

Figure 2.13: Collapse of steel framed structure due to impact of aircraft using the ASI-Gauss technique for analysis [50]

adaptively controlled in both the elastic and plastic ranges. Therefore, the ASIGauss technique gave realistic results in a short calculation time; however the damping matrices and the member properties such as local buckling were not considered [51].

Papadrakakis et al. performed a seismic collapse analysis on a 22 storey framed structure using a nonlinear finite element method with the ASI-Gauss technique [52]. The collapse of the frame is shown in Figure 2.14. The code that was used included contact between members. The results showed acceptable results for a numerical estimation of the seismic design of the large-scale framed structures. The authors noted that future work is needed to consider the damping matrices, 
and more details in the material properties.

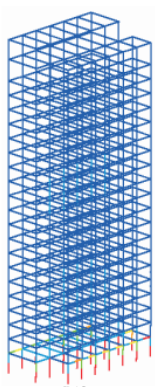

5.12 。

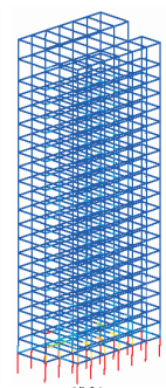

$15.04 \mathrm{~s}$
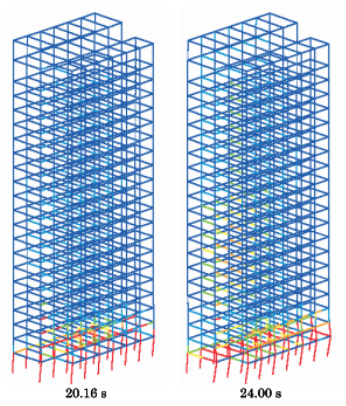
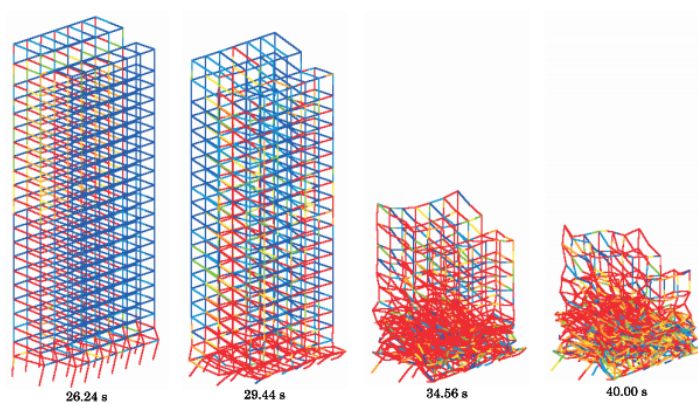

Figure 2.14: Seismic Collapse of Frame using the ASI-Gauss Technique [52]

\subsection{Applied Element Method}

The Applied Element Method (AEM), was developed by Meguro and Tagel-Din, and is based on combining FEM and DEM. In AEM the members are divided into rigid elements connected through the surface using shear and normal springs, that represent the stresses and strains within the structure. The AEM was developed to aid in the analysis of highly nonlinear behaviour of structures, such as crack initiation, crack propagation, separation of structural elements, rigid body motion of failed elements and total collapse of the structure [6]. Once the stresses in the springs exceed the ultimate yield value, the springs are disconnected and the discontinuous behaviour can be modelled. Several works have been done in this area to implement the collapse behaviour of structures. The Extreme Loading software was developed as a commercial software to analyse structures by using the AEM. The major advantages of the method come from the simplicity in the modelling analysis as well as the low CPU time. The AEM has been successful in modelling the collapse of structures under earthquake loading and blast loads, incorporating highly nonlinear behaviour, such as crack initiation, crack propagation [54].

There have been several modifications to the method as well as applications to progressive collapse of structures during the last 20 years. A commercial software was also developed using the AEM for design and modelling of structures. The commercial software is called the Extreme Loading Software (ELS). Several researchers have used the software to model structures under different loadings 
such as blast loadings, earthquakes and impact, and found relatively good results. Some of the applications using ELS are shown below.

\subsubsection{Progressive Collapse of RC structures using ELS}

Helmy, Salem, and Mourad evaluated the resistance of RC structures designed, according to the American Concrete Institute 318-08 (ACI), to progressive collapse initiated by the loss of a primary vertical support [55]. ELS was used for the analysis of a 10-storey RC frame structure, with seven equal 6 metre bays in each direction. The cases of analysis were based on the General Services Administration Code specifications and were; removal of a corner column, removal of an edge column, removal of an internal column, removal of another internal column near the structure edge, removal of an edge shear wall, removal of an internal shear wall, and removal of an internal core corner. All the removals are to be taken from the ground floor.

The authors first analysed all the mentioned cases in 2D [55] . It was recommended that the consideration of debris collision should not be considered in $2 \mathrm{D}$ analysis since, it resulted in a $100 \%$ chance that collapse of the structure would occur, as shown in Figures 2.15 and 2.16.

A 3D analysis was then performed by these authors for all the cases shown in Figure 2.17. All the cases showed partial collapse except for the internal shear wall and core corner cases. These cases did not include slabs. When the slabs were added no collapse took place. This research showed that modelling collapse cases using the ELS was an efficient way and code requirements were met.
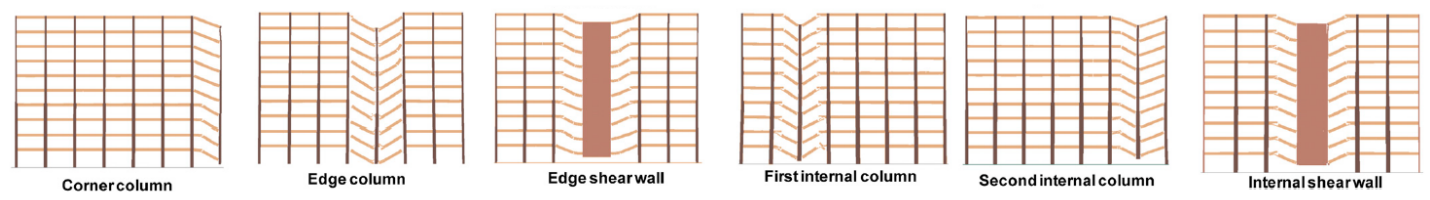

Figure 2.15: 2D Frame analysis [55]
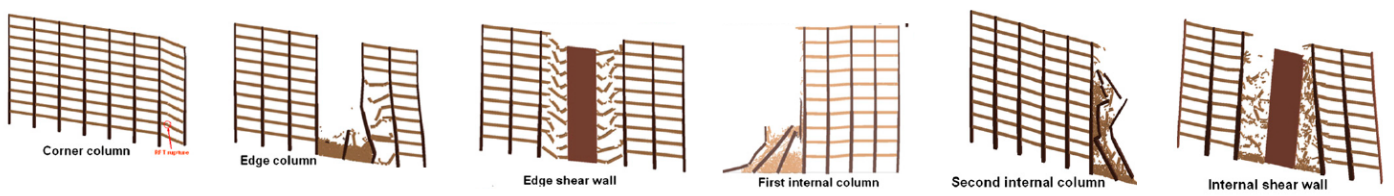

Figure 2.16: 2D Frame analysis while considering debris collision [55] 

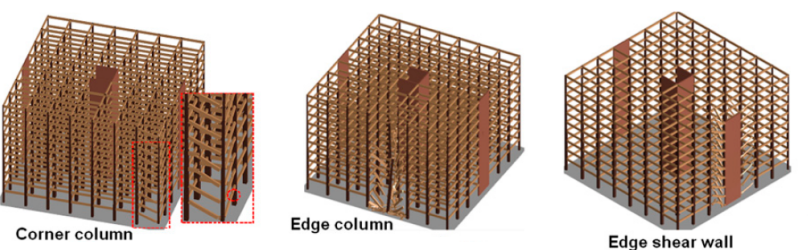

Edge shear wall

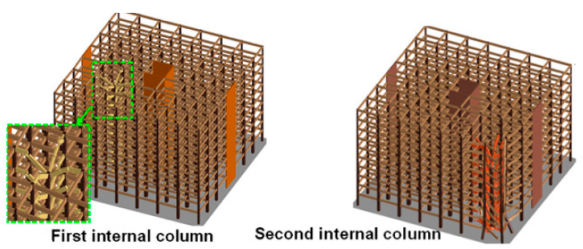

Figure 2.17: 3D Frame analysis [55]

Rahman, Elfouly, and Booth used the alternate path method to analyse a 5story building for progressive collapse based on the criteria of the Department of Defence [56]. Two different wall sections were removed for the analysis. The ELS was used for the analysis for a 3D nonlinear dynamic analysis. The results showed that composite deck floor slabs were able to bridge over the removed wall sections. Re-distribution of gravity loads was achievable from the slabs to the adjacent wall components. The study provided an understanding of the composite deck floor-cold-formed steel stud bearing walls building system in resisting progressive collapse [56].

Lupoae and Bucur modelled the controlled demolition of a 6-storey reinforced concrete frame [57] using the ELS software. In order to demonstrate a demolition, several steps needed to take place. The sequence in which the elements destruction had to be presented, as well as the time of analysis and time step. Figure 2.18 displays images of the demolition of the building at different stages, compares to the ELS software generated results. The results show good accordance with the real collapse.

As well as using the AEM and its accompanying ELS for modelling structures, researchers have implemented modifications to the method in order to increase its usability and performance. Those modifications are explained in the following sections.

\subsubsection{Improved Applied Element Method}

AEM has shown high accuracy; however, it is hard to handle non-rectangular cross-sections. The Improved Applied Element Method [58], IAEM, is therefore introduced by Elkholy, Tagel-Din, and Meguro to handle steel materials such as I-beam cross-sections, and U-channels. This was done by using two extensions 

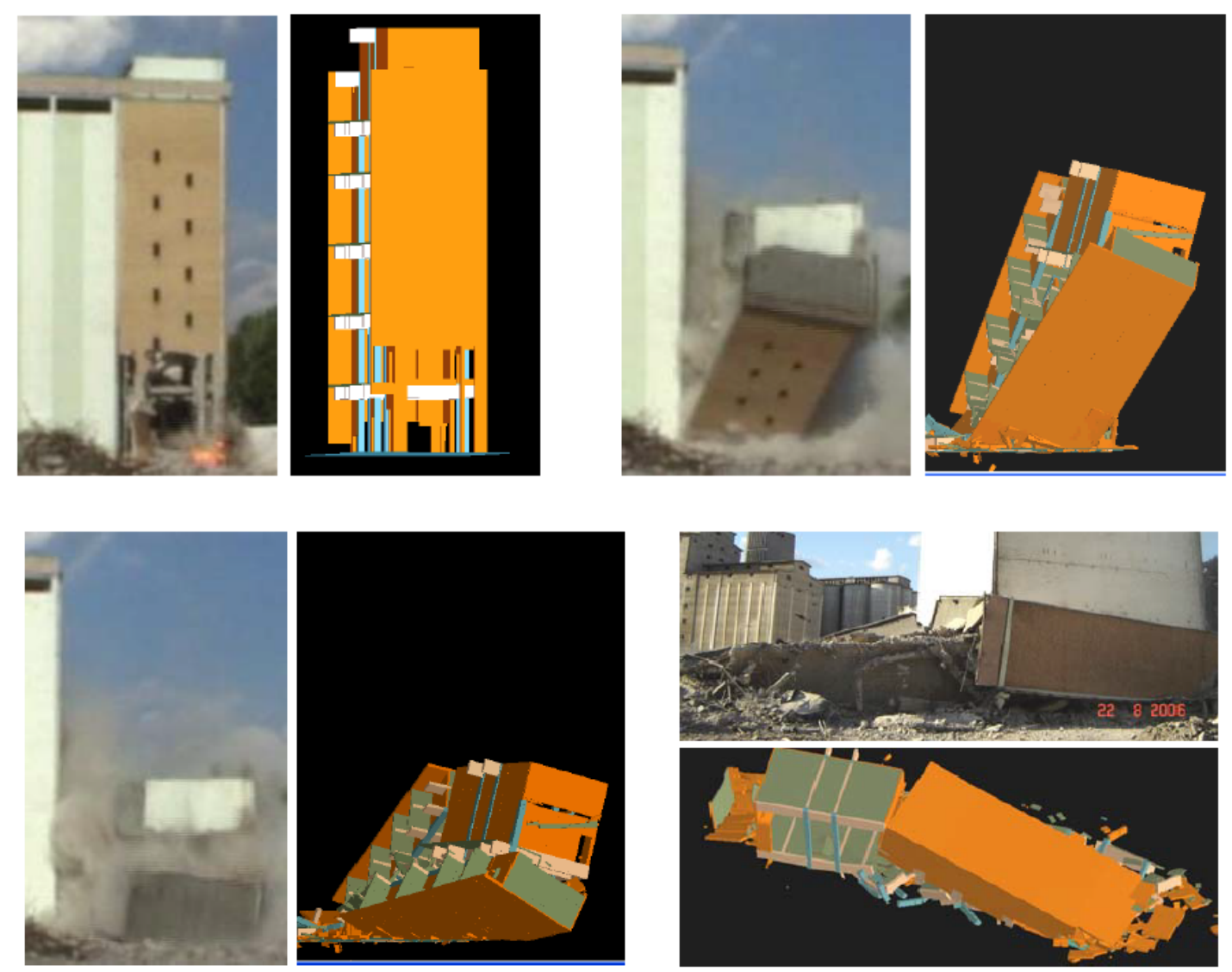

Figure 2.18: Comparison of Real Demolition vs ELS [57]

of the AEM: (1) improving the element type to follow any change in the nonrectangle cross-section thickness and (2) allowing different thickness to be used for calculating normal and shear stiffness [58].

A steel beam with an I cross-section was also analysed by the authors [58]. The results of both AEM and IAEM deflection results are shown in Table 2.1. The results showed that the number of elements required in IAEM is significantly lower than in AEM, as well as the DOF required, with a low change in error difference. This shows that the IAEM can get almost the same results as AEM but 120 times faster and with a model that is 355 times smaller. The error percentage is the difference with the solution obtained from AEM and IAEM compared to the analytical solution.

Table 2.1: Comparison AEM vs IAEM steel beam

\begin{tabular}{c|ccccc} 
& No. elements & No. DOF & CPU Time [sec] & Deflection [mm] & Error $\%$ \\
\hline AEM & 6040 & 17940 & 120.1 & 0.9003 & +2.31 \\
IAEM & 17 & 45 & $>1$ & 0.8657 & -1.59
\end{tabular}


A dynamic analysis of a 15 storey 2D frame was also performed. The columns are I-beams with the same cross-section. Using the IAEM, 870 elements were needed, while 543,750 elements were needed using conventional AEM. The results showed that the first 8 modes of vibration of the structure had less than $1.5 \%$ difference with the conventional AEM. This shows that almost the same results were obtained using a significantly different number of elements.

Finally, the collapse analysis of a 30 storey frame structure. The structure was subjected to a localized failure due to fire effect, modelled by changing the steel material properties for the members undergoing fire. Figure 2.19 displays the progressive collapse of the structure.

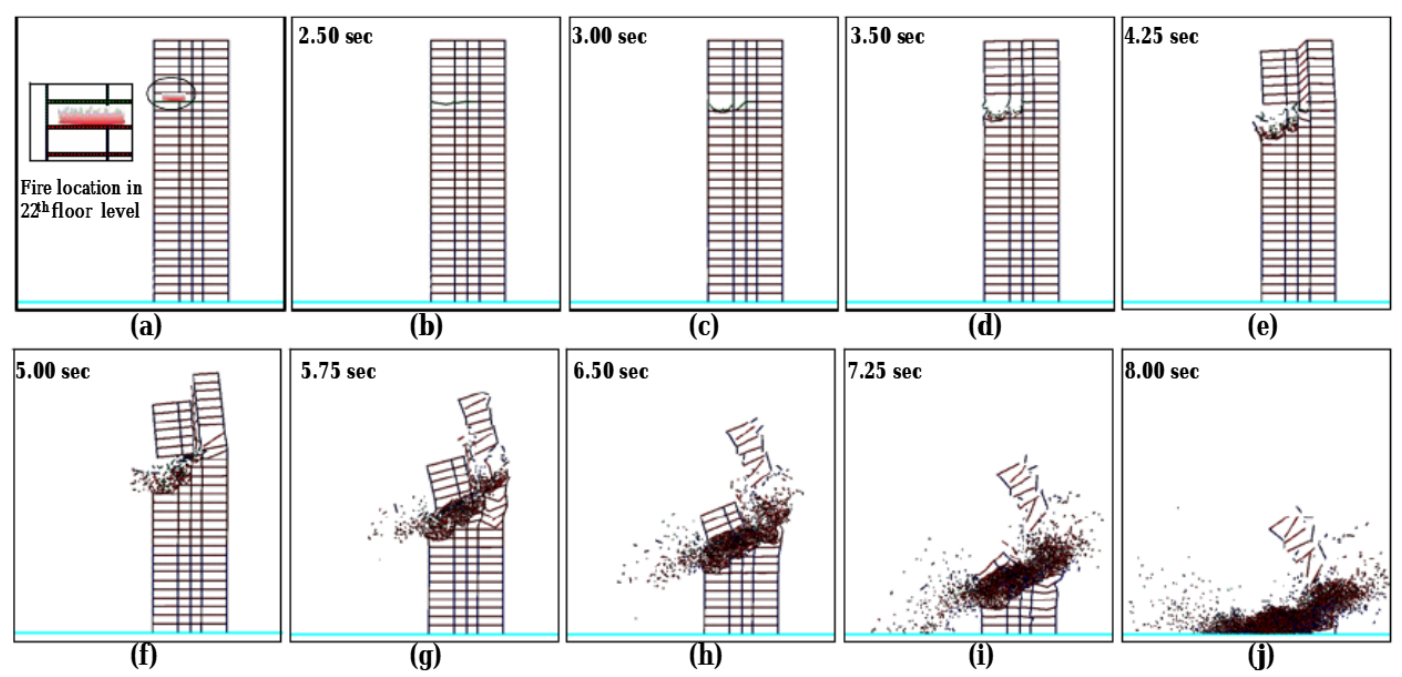

Figure 2.19: IAEM frame collapse [58]

\subsubsection{Voronoi Applied Element Method}

An AEM based on Voronoi shape was developed, where the elements are based on the Voronoi tessellation [59]. Representing the domain within the elements is done by associating the locations of the elements nodes in the physical domain, with the closest member(s) of the element nodal set with respect to Euclidean distance. Using the Voronoi Applied Element Method (VAEM), the element nodes can be placed in the physical domain without any constraint. This gives the advantage of being able to fit any domain shape without considerably reducing the element size in the original AEM. The advantages of the method were that the domain was easy to fit, the numerical Poisson's ratio is not required, varying the element 
size is easy to implement and finally, the displacement solution is independent of element size. The formulation of the method is displayed in the next chapter.

A simulation of a reinforced concrete beam was carried out by Worakanchana and Meguro [59]. Figure 2.20 shows the crack of the beam from the analysis using VAEM. By comparing the crack patterns from the VAEM, the AEM and the actual damage, it was observed that crack patterns from the VAEM were closer to the actual crack patterns rather than the AEM.

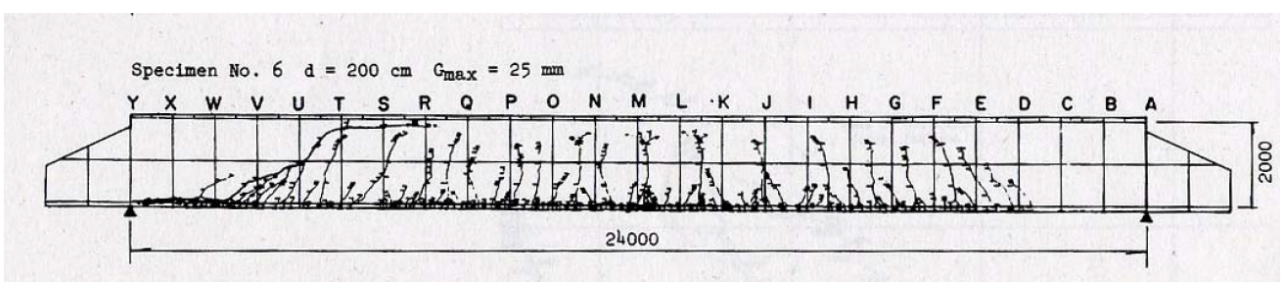

(a) Experimental crack pattern [59]

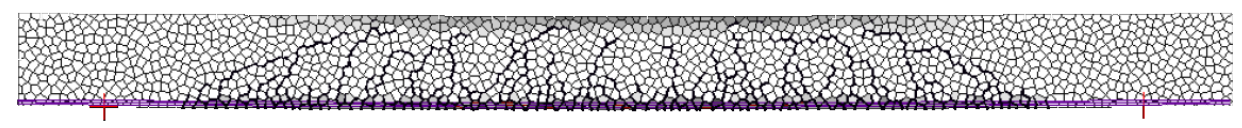

(b) VAEM crack pattern [59]

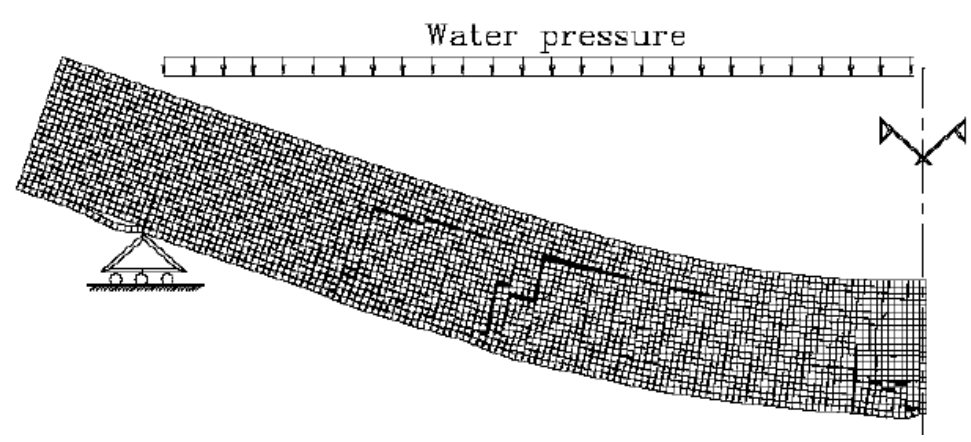

(c) AEM crack pattern [59]

Figure 2.20: Comparison of crack pattern of beam [59]

\subsection{Summary of Methods and Conclusion}

Different methods that have been used in modelling the progressive collapse of structures were presented in this chapter. The advantages and disadvantages of the methods mentioned are summarized in Table 2.2. 
Table 2.2: Summary of Advantages and disadvantages of Methods

\begin{tabular}{|c|c|c|}
\hline Method & Advantages & Disadvantages \\
\hline FEM & Accurate in continuum phase & $\begin{array}{l}\text { Cannot model element separa- } \\
\text { tion }\end{array}$ \\
\hline DEM & Can model element separation & Not efficient in continuum \\
\hline New DEM & Can model continuum behaviour & $\begin{array}{l}\text { New method should be coupled } \\
\text { with original method }\end{array}$ \\
\hline FEM-DEM & $\begin{array}{l}\text { Can model collapse of structures } \\
\text { by using FEM for small displace- } \\
\text { ments and DEM for large dis- } \\
\text { placements }\end{array}$ & Complexity in coupled equations \\
\hline MDEM & $\begin{array}{l}\text { Can model continuous media - } \\
\text { such as concrete }\end{array}$ & $\begin{array}{l}\text { Explicit - requires large compu- } \\
\text { tational time }\end{array}$ \\
\hline EDEM & Can model collapse of structures & $\begin{array}{l}\text { Explicit - requires large compu- } \\
\text { tational time }\end{array}$ \\
\hline RBSM & $\begin{array}{l}\text { Can analyse structures undergo- } \\
\text { ing cracking or sliding. Suitable } \\
\text { for plastic collapse analysis using } \\
\text { plastic hinges, hinges lines and } \\
\text { slip lines }\end{array}$ & $\begin{array}{l}\text { The accuracy and convergence } \\
\text { of the linear elastic solutions are } \\
\text { not guaranteed. The effects of } \\
\text { rotation are not taken into ac- } \\
\text { count. }\end{array}$ \\
\hline ASI-Gauss & $\begin{array}{l}\text { Gives realistic results in short } \\
\text { period of time }\end{array}$ & $\begin{array}{l}\text { Damping matrices are not con- } \\
\text { sidered. }\end{array}$ \\
\hline AEM & $\begin{array}{l}\text { Can model collapse of struc- } \\
\text { tures. accurate in continuum } \\
\text { and discontinuous phase }\end{array}$ & - \\
\hline
\end{tabular}

Table 2.3 shows what the formulations of the methods are based on. The FEM and DEM are considered to have been original formulations, and the remainder of the methods are formulated based on improvements or modifications to the methods. The RBSM, although based on the FEM was one of the first methods to use springs between elements to represent discrete behaviour.

From this review it is clear that there were three different approaches to modelling the collapse of structures behaviour. The first is by combining the finite element method and the discrete element method, where the finite element method was used for the continuum phase, and the discrete element method models in the discontinuous phase.

Another approach that was found in most of the presented solution models is the use of springs between elements. The rigid bodies spring model was one of the 
Table 2.3: Summary of methods formulation basis

\begin{tabular}{l||l}
\multicolumn{1}{l||}{ Method } & Formulation \\
\hline \hline FEM & Original \\
\hline Discrete element method & Original \\
\hline FEM-DEM & $\begin{array}{l}\text { Coupling between FEM and } \\
\text { DEM }\end{array}$ \\
\hline New Distinct Element method & Based on DEM \\
\hline RBSM & Original Springs based method \\
\hline EDEM & Based on RBSM and DEM \\
\hline MDEM & Based on RBSM and DEM \\
\hline ASI-Gauss & $\begin{array}{l}\text { Based on FEM and use of plastic } \\
\text { hinges }\end{array}$ \\
\hline AEM & Based on RBSM
\end{tabular}

first methods to use springs between elements for analysis of collapse behaviour. The method is based on using springs between rigid elements. When the elements springs fail, the elements behave as discrete elements. The extended distinct element method used pore springs between rigid bodies. The disadvantage of the method is that it required large computational cost. A modification to the method was presented by implementing the lattice element model in the extended distinct element method. The method successfully modelled the collapse of reinforced concrete structures, but further verifications to the method were required. The modified distinct element method also used pore springs between elements to model the progressive collapse behaviour of structures. The applied element method can accurately model the behaviour of a structure from no loading to total failure, since the elements can be separated and act both as continuum when connected together with springs, and discrete when they separate.

The final approach, presented in the shifted integration technique, is based on finding the exact location of plastic hinges.

It is clear that the methods are related to one another and have been derived by creating different modifications that allowed the presence of different types of results. In the next chapter the formulation of the methods is presented. 


\section{Chapter 3}

\section{Numerical Methods Approaches}

This chapter is a summary of the formulations of some methods mentioned in Chapter 2. The purpose is to demonstrate the evolution of the formulations, to have a full understanding of the method used for modelling the collapse of structures. Also, it is important to comprehend the difference between the computational algorithms of the methods for a clearer view on the advantages and disadvantages of each as well as their relationships to one another. The methods formulations presented in this chapter are the finite element method, the discrete model for continuum, the Lattice model using the Extended distinct element method, the rigid bodies spring models, and the shifted integration technique. The formulation of the Applied Element Method is presented in the next chapter.

\subsection{Mechanics of Continuous Bodies}

Before describing the numerical methods, a couple of principles must first be introduced. The mathematical models of structural problems are generated from differential equations that satisfy the whole domain. These equations are formulated from the three fundamental laws of mechanics; conservation of mass, conservation of linear momentum, and conservation of angular momentum [60]. For Lagrangian description of problems, the conservation of mass is satisfied and the conservation of angular momentum is symmetric, leaving the conservation of linear momentum to be the leading equation that must satisfy the force equilib- 
rium. The principle of conservation of linear momentum can be derived either by using Newton's Second Law, or the principle of virtual displacements [61]. The use of Newton's laws required the isolation of a volume element of a structure with all its applied and reactive forces. The sum of the static and dynamic forces and moments acting on the element is then set to zero in order to retrieve the equations of motion. For a simple mechanical system, this approach may be viable since the free-body diagram can be set up, and it is a simple way to derive the governing equations. However, this approach is more inconvenient for more complicated systems, and the type of boundary conditions are not always clear [61]. The energy principle is one of the simplest theories that can formulate a structural problem, since, if the structural system is in equilibrium, then it has potential energy [60]. In the energy approach the total work done in the volume element due to the actual forces moving through the virtual displacements that are consistent with the geometric constraints are set to zero to obtain the equations of motion [61]. The energy approach produces both the equations of motions and the force boundary conditions, as well as the form of the variables related to the boundary conditions. The energy expressions are also effective in obtaining approximate solutions by direct variational methods, such as the finite element method [61].

\subsubsection{External and Internal Virtual Work}

The work done by a force that acts on a material point and moves through a displacement, is defined by the projection of the force in the direction of the displacement times the magnitude of the displacement [61]. The work done through virtual displacements is called virtual work. The virtual work done by actual forces $F$ in a body $\Omega$ is given by Equation 3.1.

$$
\delta W=\int_{\Omega} F \cdot \delta u d v
$$

where $d v$ is the volume element in the material body $\Omega$ [61], $\delta u$ is the virtual displacement. The virtual work done by virtual forces moving through the actual 
displacement $u$ is;

$$
\delta W^{*}=\int_{\Omega} \delta F \cdot \delta u d v
$$

The virtual work done by externally applied forces moving through their respective virtual displacements is called the external virtual work denoted by $\delta W_{E}$. Similarly, the virtual work done by internal forces moving though the virtual displacements is called internal virtual work, denoted by $\delta W_{I}$.

\section{External Virtual Work}

The external virtual work due to virtual displacements $\delta u$ in a body $\Omega$ subjected to body forces f per unit volume and surface tractions $\mathrm{T}$ per unit area of the boundary $\Gamma_{\Omega}$ is given by 3.3 .

$$
\delta W_{E}=-\left(\int_{\Omega} f \cdot \delta u d v+\int_{\Gamma_{\Omega}} T \cdot \delta u d s\right)
$$

where $d s$ denotes the surface element. Th first integral extends over the volume of the body, while the second integral extends over the surface of the body. The negative sign indicates that the work is performed on the body. The external virtual work done due to virtual body forces is given by;

$$
\delta W_{E}^{*}=-\left(\int_{\Omega} \delta f \cdot u d v+\int_{\Omega_{u}} \delta_{t} \cdot u d s\right)
$$

\section{Internal Virtual Work}

Internal stresses are experienced in bodies when they deform due to forces applied. The movement of the particles in the body can be represented in terms of strains [61]. The internal virtual work done due to the virtual displacement can be computed. Assuming an infinitesimal material element of volume $d v=d x_{1} d x_{2} d x_{3}$, experiencing small virtual strains $\delta \varepsilon_{i j}$, shown in Equation 3.5 due to the virtual displacements $\delta u_{i}$; the work done by the force due to the actual stress $\sigma_{11}$, in 
moving through the virtual displacement $\delta u_{1}=\delta \varepsilon_{11} d x_{1}$ is shown in Equation 3.9.

$$
\begin{aligned}
\delta \varepsilon_{i j} & =\frac{1}{2}\left(\frac{\partial \delta u_{i}}{\partial x_{j}}+\frac{\partial \delta u_{j}}{\partial x_{i}}\right) \\
\left(\sigma_{11} d x_{2} d x_{3}\right)\left(\delta \varepsilon_{11} d x_{1}\right) & =\sigma_{11} \delta \varepsilon_{11} d x_{1} d x_{2} d x_{3}
\end{aligned}
$$

The work done by the force due to stress $\sigma_{12}$ in the shearing body from Figure 3.1 is

$$
\left(\sigma_{12} d x_{2} d x_{3}\right)\left(2 \delta \varepsilon_{12} d x_{1}\right)=2 \sigma_{12} \delta \varepsilon_{12} d x_{1} d x_{2} d x_{3}
$$

Therefore the total virtual work done by the forces due to all the stresses is

$$
\left(\sigma_{11} \delta \varepsilon_{11}+\sigma_{22} \delta \varepsilon_{22}+\sigma_{33} \delta \varepsilon_{33}+2 \sigma_{12} \delta \varepsilon_{12}+2 \sigma_{13} \delta \varepsilon_{13}+2 \sigma_{23} \delta \varepsilon_{23}\right) d x_{1} d x_{2} d x_{3}=\sigma_{i j} \delta \varepsilon_{i j} d v
$$

The total internal virtual work done $\delta W_{I}$ is obtained by integrating Equation 3.8 over the volume of the body, giving

$$
\delta W_{I}=\int_{\Omega} \sigma_{i j} \delta \varepsilon_{i j} d v=\int_{\Omega} \sigma: \delta \varepsilon d v
$$

where ":" is the double dot product.
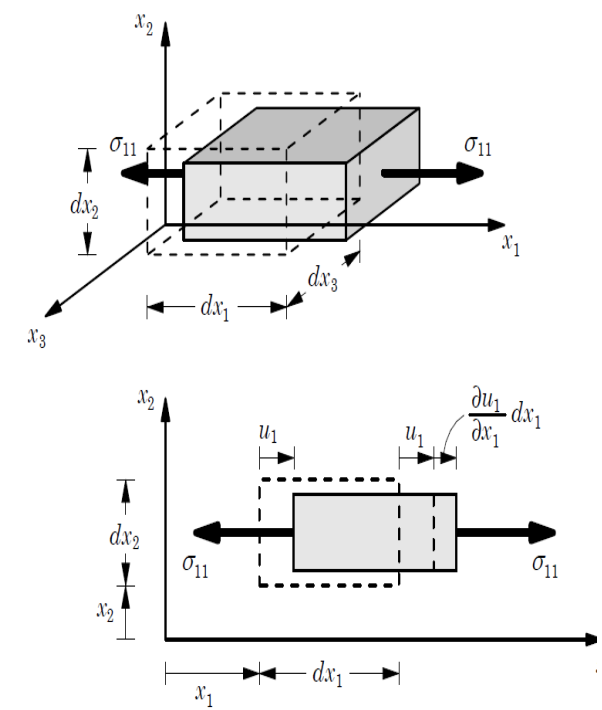

(a) by shear stress $\sigma_{11}$
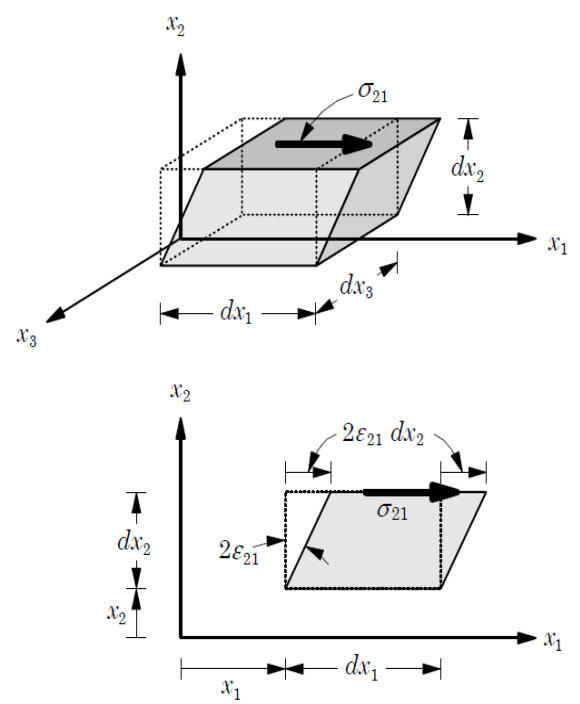

(b) by shear stress $\sigma_{21}$

Figure 3.1: Virtual Work Done [61] 


\subsection{Energy Principle Equations}

\subsubsection{The Principle of Virtual Displacements}

The virtual work due to virtual displacements is the work done by the actual forces required in moving particles of a body through virtual displacements. Considering a rigid body with applied forces $F_{1}, F_{2}, .$. , and the points subject to those forces undergo virtual displacements $\delta u_{1}, \delta u_{2}, \ldots$, respectively. The external virtual work done by the virtual displacements in given by Equation 3.10 [61].

$$
\delta W_{E}=-\left[F_{1} \cdot \delta u_{1}+F_{2} \cdot \delta u_{2}+\ldots+F_{n} \cdot \delta u_{n}\right]=-F_{i} \cdot \delta u_{i}
$$

The internal virtual work done $\delta W_{I}=\delta U$ is zero because a rigid body does not undergo any strains. Thus,

$$
\delta W_{E}=-F_{i} \cdot \delta u_{i}=-\left(\sum_{i=1}^{n} F_{i}\right) \cdot \delta u \quad \text { and } \quad \delta W_{I}=0
$$

Now considering a body $\beta$ in equilibrium under body forces $\mathbf{f}$ and boundary forces $\mathbf{T}$, and the initial configuration of the body $C^{0}$, with volume denoted by $\Omega$. $\mathbf{u}$ is the displacement vector corresponding to the equilibrium configuration of the body, and $\sigma$ and $\varepsilon$ are the stress and strain tensors respectively. In order to determine the equations that govern the equilibrium configuration $\mathbf{C}$, the body should experience a virtual displacement $\delta u$ from the true configuration. The principle of virtual work can be stated as: If a continuous body is in equilibrium, the virtual work of all actual forces in moving through a virtual displacement is zero, given by Equation (3.12)

$$
\delta W_{I}+\delta W_{E}=\delta W=0
$$

The principle of virtual displacements for deformable body can be expressed as Equation (3.13)

$$
\int_{\Omega} \sigma: \delta \varepsilon d v-\int_{\Omega} \rho f \cdot \delta u d v-\int_{\Gamma \sigma} T \cdot \delta u d s=0
$$




\subsubsection{The Principle of Minimum Potential Energy}

Consider a body in static equilibrium under an applied force. Due to the load, the structure experiences deformation, described by $u(x)=\left[u_{1}, u_{2}, u_{3}\right]^{T}$. The structure resists deformation by generating internal forces, where each internal force is proportional to the amount of deformation. If the internal force is smaller than the load, then the structure continues to deform to equilibrate the forces. The internal force generated during deformation may be considered as the energy stored in the structure. As the structure deforms, the internal force increases as well as the energy in the structure. The stored energy is referred to as the strain energy and is shown in Equation 3.14.

$$
U(u)=\frac{1}{2} \iint_{\Omega} \sigma(u): \varepsilon(u) d \Omega
$$

where the components of the strain tensor are defined as;

$$
\varepsilon_{i j}(u)=\frac{1}{2}\left(\frac{\delta u_{i}}{\delta x_{j}}+\frac{\delta u_{j}}{\delta x_{i}}\right)=\frac{1}{2}\left(u_{i, j}+u_{j, i}\right)
$$

where $u_{i, j}=\delta u_{i} / \delta x_{j}$. The strain energy $\mathrm{U}(\mathrm{u})$ is the energy required to produce the displacement . Since $U(u)$ does not depend on the deformation path in elastic problems, it is a function of the displacement only. If the forces are applied to the structure, and the deformation is in the direction of the applied forces, then the work is done by those applied forces, and can be defined as

$$
W(u)=\iint_{\Omega} u \cdot f d \Omega
$$

If the applied force is conservative(the load is independent of deformation), then the work done is a negative value of the potential energy generated by the loads. And since the strain energy $\mathrm{U}(\mathrm{u})$ is independent of the path, it is the potential energy that is stored in the structure. The potential energy is the difference between the strain energy and the work done, and is written as

$$
\Pi(u)=U(u)-W(u)=\frac{1}{2} \iint_{\Omega} \sigma(u): \varepsilon(u) d \Omega-\iint_{\Omega} u \cdot f d \Omega
$$




\subsection{Finite Element Method}

Many problems are expressed in partial differential equations that cannot be solved with analytical methods. Thus, an approximation is required, and can be based on different types of discretisation. The discretisation methods approximate the differential equations with numerical model equations, which can be solved using numerical methods. Since the solution can have a complicated expression, the finite element can be used [62].

Figure 3.2 is used to interpolate the two nodes $\xi_{1}$ and $\xi_{2}$. The element is the segment between the two nodes, and the values of the function are $f_{1}$ and $f_{2}$. Approximating the function at an arbitrary point in the element gives

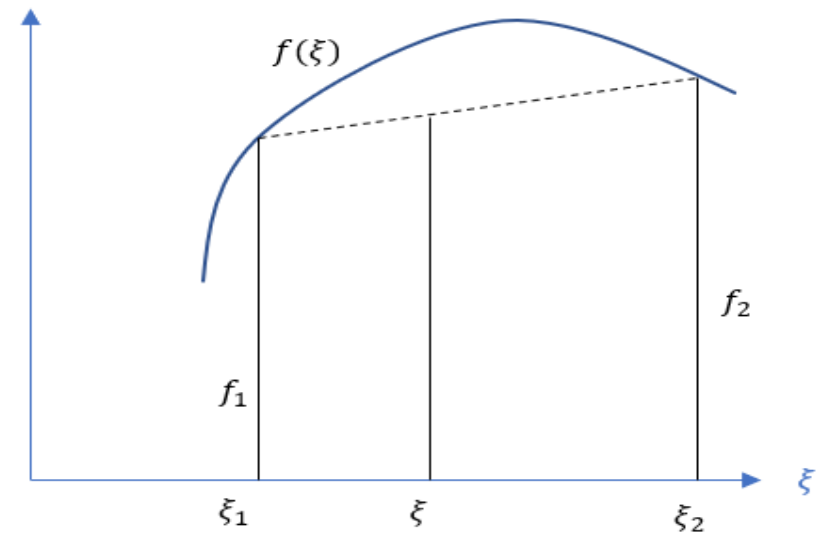

Figure 3.2: Interpolation of a one-variable function

$$
f(\xi)=\frac{\xi_{2}-\xi}{\xi_{2}-\xi_{1}} f_{1}+\frac{\xi-\xi_{1}}{\xi_{2}-\xi_{1}} f_{2}
$$

The interpolation can be written in the form

$$
f(\xi)=N_{1}(\xi) f_{1}+N_{2}(\xi) f_{2}
$$

where $N_{1}$ and $N_{2}$ are functions of $\xi$ and are known as the shape functions. The shape functions must satisfy the following conditions

$$
\begin{array}{ll}
N_{1}\left(\xi_{1}\right)=1, & N_{1}\left(\xi_{2}\right)=0 \\
N_{2}\left(\xi_{1}\right)=1, & N_{2}\left(\xi_{2}\right)=0
\end{array}
$$


The shape functions are shown in Figure 3.3.

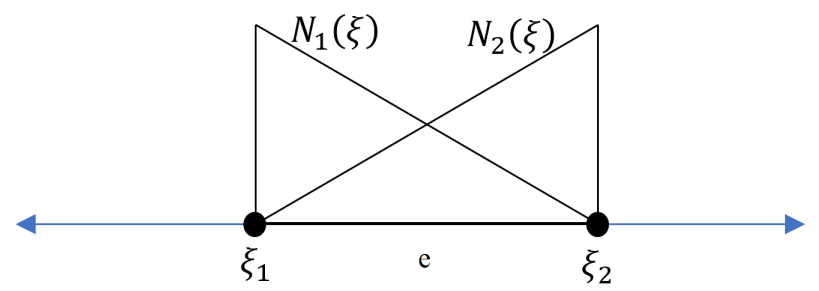

Figure 3.3: 1D finite element $e$ with shape functions

\subsubsection{Isoparametric mapping}

A solid component in the $(x, y)$ plane should be divided into finite elements. For the scope of this thesis quadrilateral elements are considered. First, a unit square on plane $(\xi, \eta)$ must be mapped to a quadrilateral on plane $(x, y)$. The unit square is a reference element. It is necessary to use a reference element so that different shape functions are not needed to be built for different elements. Assume the four nodes of the quadrilateral are labelled as 1,2,3,4 in the counter-clockwise direction. Thus on the $(x, y)$ plane the four nodes have coordinates $\left(x_{1}, y_{1}\right)$, $\left(x_{2}, y_{2}\right),\left(x_{3}, y_{3}\right),\left(x_{4}, y_{4}\right)$. The quadrilateral in both planes is shown in Figure 3.4. Mapping a point in the $(\xi, \eta)$ to a point in the $(x, y)$ plane gives

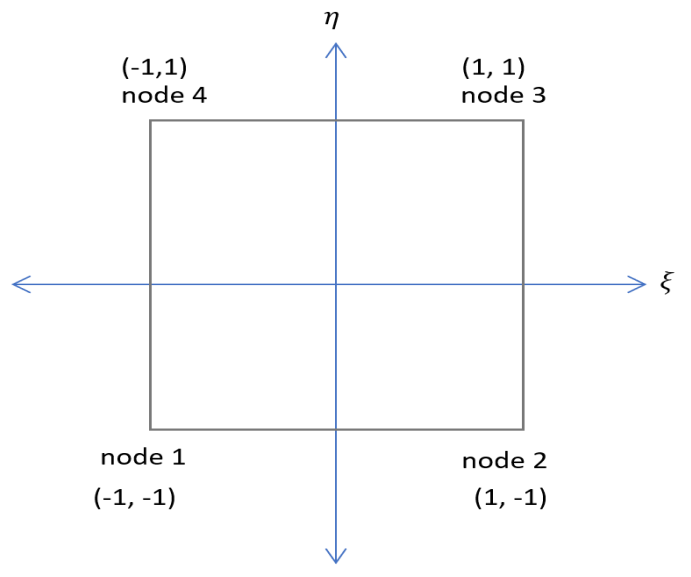

(a) $\xi, \eta$ plane

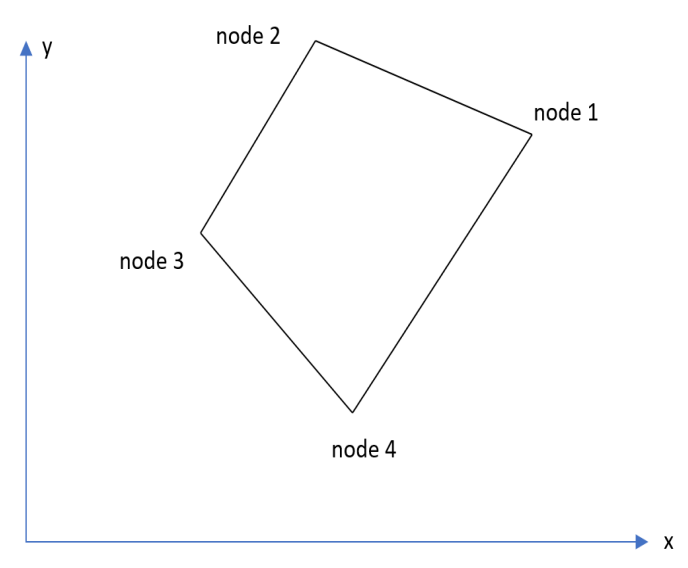

(b) $x, y$ plane

Figure 3.4: Quadrilateral in different planes

$$
\begin{gathered}
x=N_{1} x_{1}+N_{2} x_{2}+N_{3} x_{3}+N_{4} x_{4} \\
y=N_{1} y_{1}+N_{2} y_{2}+N_{3} y_{3}+N_{4} y_{4}
\end{gathered}
$$


Equation 3.22 can be written as

$$
x(\xi)=\sum_{i=1}^{n} N_{i}(\xi) x_{i}
$$

where $n$ is the number of nodes in the element, for a linear quadrilateral $n=4$. Now the shape functions must be determined. On the plane $(\xi, \eta), N_{1}$ should be 1 at node 1 and zero at the other three nodes. The four shapes functions are

$$
\begin{aligned}
& N_{1}=\frac{1}{4}(1-\xi)(1-\eta) \\
& N_{2}=\frac{1}{4}(1+\xi)(1-\eta) \\
& N_{3}=\frac{1}{4}(1+\xi)(1+\eta) \\
& N_{4}=\frac{1}{4}(1-\xi)(1+\eta)
\end{aligned}
$$

The mapping is called isoparametric mapping because the shape functions are used for interpolating geometry and displacements [60]. The displacements of the four nodes are presented in vector $\mathbf{q}$

$$
\begin{aligned}
\mathbf{q} & =\left[\begin{array}{llllllll}
u_{1} & v_{1} & u_{2} & v_{2} & u_{3} & v_{3} & u_{4} & v_{4}
\end{array}\right]^{T} \\
\mathbf{N} & =\left[\begin{array}{ccccccccc}
N_{1} & 0 & N_{2} & 0 & N_{3} & 0 & N_{4} & 0 \\
0 & N_{1} & 0 & N_{2} & 0 & N_{3} & 0 & N_{4}
\end{array}\right]
\end{aligned}
$$

The displacement vector is

$$
\begin{array}{r}
\mathbf{u}=\mathbf{N q} \\
u(\xi)=\Sigma N_{i}(\xi) u_{i}
\end{array}
$$

Representing the strain field in terms of the nodal displacements, $\boldsymbol{\varepsilon}=\mathbf{B q}$, where $\mathbf{B}$ is a matrix depending on $(\xi, \eta)$. 


\subsubsection{Jacobian of the Mapping}

The displacement gradient on the $(\xi, \eta)$ plane is

$$
\left[\begin{array}{c}
\frac{\partial u}{\partial \xi} \\
\frac{\partial u}{\partial \eta}
\end{array}\right]=\frac{1}{4}\left[\begin{array}{llll}
-(1-\eta) & (1-\eta) & (1+\eta) & -(1+\eta) \\
-(1-\xi) & (1-\xi) & (1+\xi) & -(1+\xi)
\end{array}\right]\left[\begin{array}{l}
u_{1} \\
u_{2} \\
u_{3} \\
u_{4}
\end{array}\right]
$$

Using the chain rule for converting the gradient on $(\xi, \eta)$ to $(x, y)$

$$
\left[\begin{array}{l}
\frac{\partial u}{\partial \xi} \\
\frac{\partial u}{\partial \eta}
\end{array}\right]=\left[\begin{array}{ll}
\frac{\partial x}{\partial \xi} & \frac{\partial y}{\partial \xi} \\
\frac{\partial x}{\partial \eta} & \frac{\partial y}{\partial \eta}
\end{array}\right]\left[\begin{array}{l}
\frac{\partial u}{\partial x} \\
\frac{\partial u}{\partial y}
\end{array}\right]
$$

The two by two matrix is the Jacobian matrix $\mathbf{J}$ of the map $(\xi, \eta)$ to $(x, y)$. The elements of the Jacobian matrix are calculates as

$$
\begin{aligned}
J_{11} & =\frac{\partial x}{\partial \xi}=\frac{1}{4}(1-\eta)\left(-x_{1}+x_{2}\right)+\frac{1}{4}(1+\eta)\left(x_{3}-x_{4}\right) \\
J_{12} & =\frac{\partial y}{\partial \xi}=\frac{1}{4}(1-\eta)\left(-y_{1}+y_{2}\right)+\frac{1}{4}(1+\eta)\left(y_{3}-y_{4}\right) \\
J_{21} & =\frac{\partial x}{\partial \eta}=\frac{1}{4}(1-\xi)\left(-x_{1}+x_{4}\right)+\frac{1}{4}(1+\xi)\left(-x_{2}+x_{3}\right) \\
J_{22} & =\frac{\partial y}{\partial \eta}=\frac{1}{4}(1-\xi)\left(-y_{1}+y_{4}\right)+\frac{1}{4}(1+\xi)\left(-y_{2}+y_{3}\right)
\end{aligned}
$$

Using Cramer's rule to find $\frac{\partial u}{\partial x}$ and $\frac{\partial u}{\partial y}$ from Equation 3.33, gives

$$
\begin{aligned}
& {\left[\begin{array}{l}
\frac{\partial u}{\partial x} \\
\frac{\partial u}{\partial y}
\end{array}\right]=\frac{\left|\begin{array}{ll}
\frac{\partial u}{\partial \xi} & \frac{\partial y}{\partial \xi} \\
\frac{\partial u}{\partial \eta} & \frac{\partial y}{\partial \eta}
\end{array}\right|}{\left|\begin{array}{ll}
\frac{\partial x}{\partial \xi} & \frac{\partial y}{\partial \xi} \\
\frac{\partial x}{\partial \eta} & \frac{\partial y}{\partial \eta}
\end{array}\right|}=\frac{\left|\begin{array}{cc}
\frac{\partial u}{\partial \xi} & J_{12} \\
\frac{\partial u}{\partial \eta} & J_{22}
\end{array}\right|}{\left|\begin{array}{ll}
J_{11} & J_{12} \\
J_{21} & J_{22}
\end{array}\right|}=\frac{1}{\operatorname{det} \mathbf{J}}\left|\begin{array}{cc}
\frac{\partial u}{\partial \xi} & J_{12} \\
\frac{\partial u}{\partial \eta} & J_{22}
\end{array}\right|} \\
& {\left[\begin{array}{c}
\frac{\partial u}{\partial x} \\
\frac{\partial u}{\partial y}
\end{array}\right]=\frac{1}{\operatorname{det} \mathbf{J}}\left(J_{22} \frac{\partial u}{\partial \xi}-J_{12} \frac{\partial u}{\partial \eta}\right)}
\end{aligned}
$$


where $\frac{\partial u}{\partial \xi}$ and $\frac{\partial u}{\partial \eta}$ are known from Equation 3.32. Similar expressions can be formed for $\frac{\partial v}{\partial x}$ and $\frac{\partial v}{\partial y}$. The strain vector is

$$
\boldsymbol{\varepsilon}=\left[\left(\frac{\partial u}{\partial x}\right),\left(\frac{\partial v}{\partial y}\right),\left(\frac{\partial u}{\partial y}+\frac{\partial v}{\partial x}\right)\right]^{T}
$$

and since

$$
\begin{array}{r}
\varepsilon=\mathbf{B q} \\
\delta \varepsilon=\mathbf{B} \delta \mathbf{q}
\end{array}
$$

The entries to the $\mathbf{B}$ matrix are

$$
B_{11}=\frac{1}{4 \operatorname{det} \mathbf{J}}\left[-J_{22}(1-\eta)+J_{12}(1-\xi)\right]
$$

Now the stress should be presented in terms of the nodal displacements. The inplane stresses are $\boldsymbol{\sigma}=\left[\sigma_{x}, \sigma_{y}, \sigma_{x y}\right]^{T}$. The stress is related to the strain as

$$
\sigma=\mathrm{D} \varepsilon
$$

Under plane strain conditions, this can be explicitly be represented as

$$
\left[\begin{array}{c}
\sigma_{x} \\
\sigma_{y} \\
\sigma_{x y}
\end{array}\right]=\frac{E}{(1+\nu)(1-2 \nu)}\left[\begin{array}{ccc}
1-\nu & \nu & 0 \\
\nu & 1-\nu & 0 \\
0 & 0 & 0.5-\nu
\end{array}\right]\left[\begin{array}{c}
\varepsilon_{x} \\
\varepsilon_{y} \\
\varepsilon_{x y}
\end{array}\right]
$$

From Equation 3.41

$$
\sigma=\mathrm{DBq}
$$

Recall the internal work done from Equation 3.9. Substituting the terms from Equation 3.42 and 3.46 , results in the internal virtual work as

$$
\partial W_{I}=\delta \mathbf{q}^{\mathbf{T}} \mathbf{k q}
$$


where $\mathbf{k}$ is an integral over the volume of the element and presented as

$$
\mathbf{k}=\int_{\Omega} \mathbf{B}^{\mathbf{T}} \mathbf{D B d} V
$$

Writing the internal virtual work in terms of the global degrees of freedom gives

$$
\partial W_{I}=\delta \mathbf{Q}^{\mathbf{T}} \mathbf{K}
$$

Recall the external virtual work, from Equation 3.3

$$
\partial W_{E}=\left(\int_{\Omega} f \cdot \delta u d v+\int_{\Gamma_{\Omega}} T \cdot \delta u d s\right)
$$

and replacing the displacement variation $\delta u=N \delta q$

$$
\begin{aligned}
& \delta W_{E}=\int_{\Omega} \delta u^{T} f d v+\int_{\Gamma_{\Omega}} \delta u^{T} T d s \\
& \delta W_{E}=\int_{\Omega} N^{T} \delta q^{T} f d v+\int_{\Gamma_{\Omega}} N^{T} \delta q^{T} T d s \\
& \delta W_{E}=\delta q^{T}\left(\int_{\Omega} N^{T} f d v+\int_{\Gamma_{\Omega}} N^{T} T d s\right)
\end{aligned}
$$

where the first integral is over the volume body, and the second over the surface body where traction is prescribed. Let

$$
F=\int_{\Omega} N^{T} f d v+\int_{\Gamma_{\Omega}} N^{T} T d s
$$

then Equation 3.52 becomes

$$
\delta W_{E}=\delta q^{T} F
$$

The principle of virtual work requires that the $\partial W_{E}=\partial W_{I}$, so

$$
\begin{array}{r}
\delta Q^{T} K Q=\delta Q^{T} F \\
K Q=F
\end{array}
$$


Large displacement can be handled in the framework. Refer to [63] for complete large displacement formulations.

\subsubsection{Differential Volume and Area}

The differential volume $d V$ is the differential area $d A$ on the $(x, y)$ plane multiplied by the thickness of the element $h$. The integration will be over each element, and then all elements will be summed. The integration will be carried out over the $\xi, \eta$ plane. Consider a rectangular infinitesimal element in the $(\xi, \eta)$ plane, defined by four point shown in Figure 3.5. The element maps to the $(x, y)$ plane. The point $(\xi, \eta)$ maps to a vector $\mathbf{x}(\xi, \eta)$ on the $(x, y)$ plane.

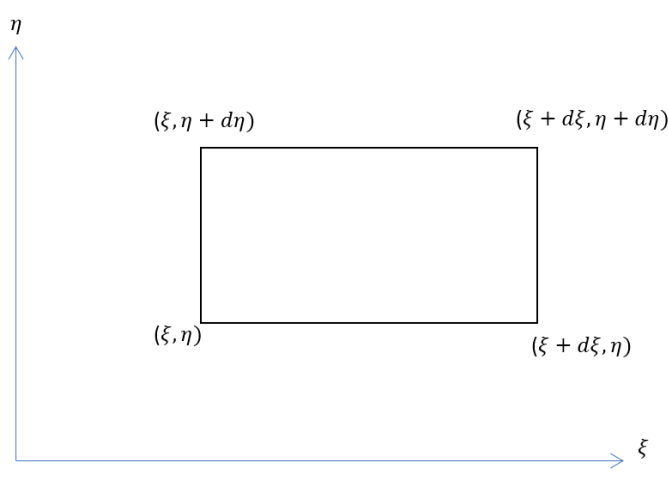

(a) on $(\xi, \eta)$ plane

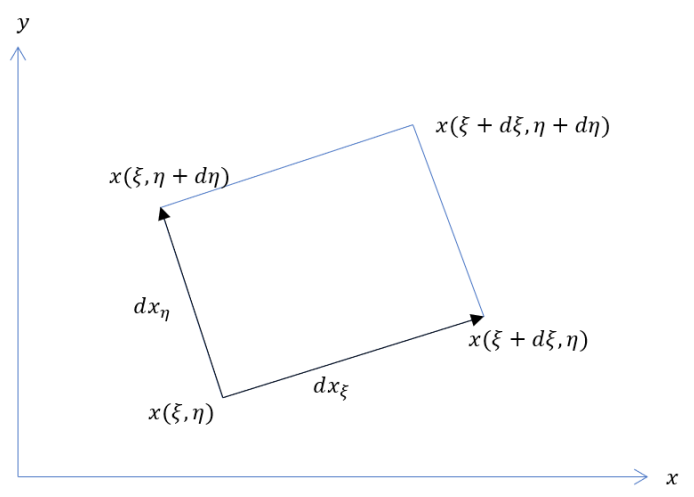

(b) on $(x, y)$ plane

Figure 3.5: Infinitesimal quadrilateral element

The quadrilateral is made of the vectors

$$
\begin{aligned}
& d_{\mathbf{x} \xi}=\mathbf{x}(\xi+d \xi, \eta)-\mathbf{x}(\xi, \eta)=\frac{\partial \mathbf{x}}{\partial \xi} d \xi \\
& d_{\mathbf{x} \mathbf{j}}=\mathbf{x}(\xi, \eta+d \eta)-\mathbf{x}(\xi, \eta)=\frac{\partial \mathbf{x}}{\partial \eta} d \eta
\end{aligned}
$$

The area of the quadrilateral is the cross product of the two vectors

$$
d A=\left|d \mathbf{x}_{\xi} \times \mathbf{x}_{\eta}\right|
$$


Calculating the cross product gives

$$
d \mathbf{x}_{\xi} \times \mathbf{x}_{\eta}=\left|\begin{array}{ccc}
\mathbf{i} & \mathbf{j} & \mathbf{k} \\
\frac{\partial x}{\partial \xi} d \xi & \frac{\partial y}{\partial \xi} d \xi & 0 \\
\frac{\partial x}{\partial \eta} d \eta & \frac{\partial y}{\partial \eta} d \eta & 0
\end{array}\right|=\mathbf{k}\left(\frac{\partial x}{\partial \xi} \frac{\partial y}{\partial \eta}-\frac{\partial x}{\partial \eta} \frac{\partial y}{\partial \xi}\right)
$$

Therefore, the area of the differential element is

$$
d A=\operatorname{det} \mathbf{J} d \xi d \eta
$$

The differential line length is

$$
d L=\left|d \mathbf{x}_{\eta}\right|=\sqrt{\left(\frac{\partial x}{\partial \eta}\right)^{2}+\left(\frac{\partial y}{\partial \eta}\right)^{2} d \eta}
$$

The volume integral then becomes

$$
\begin{aligned}
\mathbf{k} & =\int_{-1}^{1} \int_{-1}^{1} h \mathbf{B}^{\mathbf{T}} \mathbf{D B} \operatorname{det} \mathbf{J} d \xi d \eta \\
\mathbf{f}_{\mathbf{b}} & =\int_{-1}^{1} \int_{-1}^{1} h \mathbf{N}^{\mathbf{T}} \mathbf{b} \operatorname{det} \mathbf{J} d \xi d \eta \\
\mathbf{f}_{\mathbf{t}} & =d L \int_{-1}^{1} h N^{T} t d \eta
\end{aligned}
$$

where $h$ is the thickness of the element.

\subsubsection{Numerical Integration}

The finite element formulation requires solving Equations 3.63-3.65 over the domain for the construction of the element stiffness matrix and force vector [60]. Most integrals cannot be evaluated explicitly. It is usually faster to integrate them numerically rather than analytically [60]. Among numerical integration methods, the Gauss integration rule is most commonly used for its simplicity and extreme accuracy [60]. The Gauss integration method integrates polynomials with the order of $2 n_{p}-1$ exactly, with $n_{p}$ is the number of integration points.

Since the integration is carried out in the reference configuration $\xi \in[-1,+1]$, 
then all the values must be transformed to the following configuration

$$
\int_{X} g(X) d X=\int_{-1}^{+1} g(\xi) \frac{d X}{d \xi} d \xi \int_{1}^{+1} g(\xi) J(\xi) d \xi
$$

where $g(\xi)$ is the function that should be integrated, and $J$ is the Jacobian of the transformation configuration, calculated in Equations 3.34-3.37. The integration is done numerically since the product $g(\xi) J(\xi)$ is in general not a polynomial. Therefore it is approximated by the sum

$$
\int_{-1}^{+1} g(\xi) J(\xi) d \xi \approx \sum_{p=1}^{n_{p}} g\left(\xi_{p}\right) J\left(\xi_{p}\right) W_{p}
$$

where $W_{p}$ are the weighting factors and $\xi_{p}$ denote the coordinates of the evaluation points.

\subsection{Discrete Model for Continuum}

The following method is the modification that was implemented to the original discrete element method. The modification allows to model structures accurately in continuum and in discrete behaviour. The modification to the method is based on connective links between destroyed elements change to contact links of the traditional DEM and the element arrangement patterns remain the same. The DEM model for continuum dynamics problems was presented by [64] based on the principle of minimum potential energy. The method used a seven-disc model as well as an extension to a nine-disc model. The seven-disc model derivation is presented in this section.

Consider an elastic plate that is divided into rigid disc elements, connected by normal and tangential springs. Two potential compact arrangement patterns are possible; type A and type B. Type A is a seven-disc model, while type B is a nine-disc model. The model is presented in Figure 3.6. 


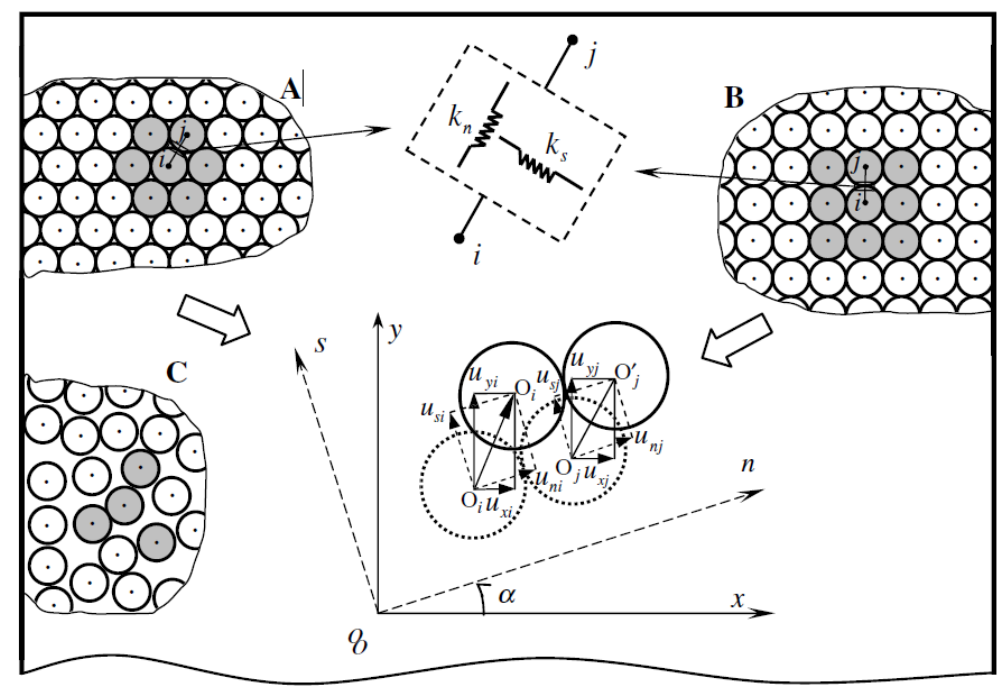

Figure 3.6: Discrete element method models based on rigid disc elements [64]

The total potential energy for the disc-spring system is

$$
\begin{aligned}
\Pi=\sum_{i}^{N}\left(U_{i} V_{i}\right)+\sum_{i}^{N}\left(u_{x i} \rho \ddot{u}_{x i} V_{i}+u_{y i} \rho \ddot{u}_{y i} V i\right) & -\sum_{i}^{N}\left(u_{x i} f_{x i} V_{i}+u_{y i} f_{y i} V_{i}\right) \\
& -\sum_{i}^{N}\left(u_{x i} \bar{T}_{x i} S_{i}+u_{y i} \bar{T}_{y i} S_{i}\right)
\end{aligned}
$$

where $U_{i}$ is the average strain energy around disc $i, V_{i}$ is the volume of the disc, $\rho$ is the mass density, $S_{i}$ is the boundary area of the external force on the disc, $u_{x i}$ and $u_{y i}$ are the displacements, $\ddot{u_{x i}}$ and $\ddot{u_{y i}}$ are the accelerations in the horizontal and vertical directions, respectively. $f_{x i}, f_{y i}$ and $\bar{T}_{x i}, \bar{T}_{y i}$ are the components of the body force and surface force in the horizontal and vertical directions, respectively.

The deformation of the body is presented through the deformation of the springs. For a disc $i$ that connects with $p$ discs, the average strain energy around disc $i$ is

$$
U_{i}=\frac{1}{V_{i}} \sum_{j}^{p} \frac{1}{2}\left[\frac{1}{2} k_{n i j}\left(u_{n j}-u_{n i}\right)^{2}+\frac{1}{2} k_{s i j}\left(u_{s j}-u_{s i}\right)^{2}\right]
$$

where $k_{n i j}$ and $k_{s i j}$ are the normal and tangential spring constants between discs $i$ and $j$. Assuming the rotation angle between the x-axes and the normal direction 
of the spring as $\alpha, l=\cos \alpha$ and $m=\sin \alpha$, then

$$
u_{n}=u_{x} l+u_{y} m, \quad u_{s}=u_{y} l-u_{x} m
$$

Substituting Equation 3.70 in 3.69 gives

$$
\begin{aligned}
U_{i}=\frac{1}{4 V_{i}} \sum_{j}^{p} k_{n i j}\left[l _ { i j } \left(u_{x j}\right.\right. & \left.\left.-u_{x} i\right)+m_{i j}\left(u_{y j}-u_{y i}\right)\right]^{2} \\
& +\frac{1}{4 V_{i}} \sum_{j}^{p} k_{s i j}\left[l_{i j}\left(u_{y j}-u_{y i}\right)-m_{i j}\left(u_{x j}-u_{x i}\right)\right]^{2}
\end{aligned}
$$

Substituting Equation 3.71 into the total potential energy Equation 3.68, and according to variational calculus $\partial \Pi / \partial u_{x i}=0, \partial \Pi / \partial u_{y i}=0, \ddot{u_{x i}}$ and $\ddot{u_{y i}}$ are given by

$$
\begin{array}{r}
\ddot{u}_{x i}=\frac{1}{\rho V_{i}}\left(f_{x i} V_{i}+\bar{T}_{x i} S_{i}+\sum_{j}^{p} k_{n i j}\left[l_{i j}^{2}\left(u_{x j}-u_{x i}\right)+l_{i j} m_{i j}\left(u_{y j}-u_{y i}\right)\right]\right. \\
+\sum_{j}^{p} k_{s i j}\left[m_{i j}^{2}\left(u_{x j}-u_{x i}\right)-l_{i j} m_{i j}\left(u_{y j}-u_{y i}\right)\right] \\
\ddot{u}_{y i}=\frac{1}{\rho V_{i}}\left(f_{y i} V_{i}+\bar{T}_{y i} S_{i}+\sum_{j}^{p} k_{n i j}\left[l_{i j} m_{i j}\left(u_{x j}-u_{x i}\right)+m_{i j}^{2}\left(u_{y j}-u_{y i}\right)\right]\right. \\
+\sum_{j}^{p} k_{s i j}\left[-l_{i j} m_{i j}\left(u_{x j}-u_{x i}\right)+l_{i j}^{2}\left(u_{y j}-u_{y i}\right)\right]
\end{array}
$$

At moment $t, \ddot{u}_{x i}$ and $\ddot{u}_{y i}$ are obtained from Equations 3.72 and 3.73. At moment $t+\Delta t$

$$
\begin{aligned}
& {\left[\dot{u}_{x i}\right]_{t+\Delta t}=\left[\dot{u}_{x i}\right]_{t}+\left[\ddot{u}_{x i}\right]_{t} \Delta t} \\
& {\left[\dot{u}_{y i}\right]_{t+\Delta t}=\left[\dot{u}_{y i}\right]_{t}+\left[\ddot{u}_{y i}\right]_{t} \Delta t} \\
& {\left[u_{x i}\right]_{t+\Delta t}=\left[u_{x i}\right]_{t}+\left[\dot{u}_{x i}\right]_{t} \Delta t} \\
& {\left[u_{y i}\right]_{t+\Delta t}=\left[u_{y i}\right]_{t}+\left[\dot{u}_{y i}\right]_{t} \Delta t}
\end{aligned}
$$

where $\Delta t$ is a time increment. 


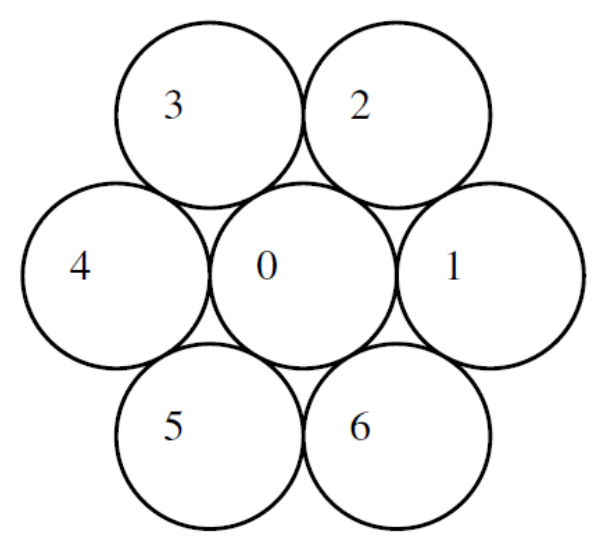

Figure 3.7: Seven-disc model

\section{Seven-disc model}

The seven-disc model is presented in Figure 3.7.

$$
\begin{array}{r}
A=r^{2}\left(2 k_{n 1}+\frac{1}{8} k_{n 2}+\frac{1 / 8}{k}\right), \quad B=r^{2}\left(\frac{9}{8} k_{n 2}+\frac{9}{8} k_{n 3}\right), \\
C_{1}=r^{2}\left(\frac{3}{8} k_{n 2}+\frac{3}{8} k_{n 3}\right), \quad C_{2}=r^{2}\left(\frac{3}{8} k_{s 2}+\frac{3}{8} k_{s 3}\right), \\
D=r^{2}\left(2 k_{s 1}+\frac{1}{2} k_{s 2}+\frac{1}{2} k_{s 3}\right), \quad E=r^{2}\left(\frac{\sqrt{3}}{8} k_{n 2}-\frac{\sqrt{3}}{8} k_{n 3}\right) \\
F=r^{2}\left(\frac{3 \sqrt{3}}{8} k_{n 2}-\frac{3 \sqrt{3}}{8} k_{n 3}\right), \quad G=r^{2}\left(-\frac{\sqrt{3}}{4} k_{s 2}+\frac{\sqrt{3}}{4} k_{s 3}\right)
\end{array}
$$

where $\delta=V /\left(2 \sqrt{3} r^{2}\right)$ is the disc thickness. For orthotropic $c_{16}=0$ and $c_{26}=0[64]$.

\subsection{Lattice Model for RC using EDEM}

The lattice model is composed of two parts; before fracture, and after fracture. A concrete element is modelled as a lattice model consisting of two lumpedmasses connected by axial truss elements as shown in Figure 3.8. The constitutive model of a concrete truss element is therefore described by a uni-axial tensile/compressive law.

The dynamic characteristics of the model after spring fracture are described by DEM. Re-contact of the fracture materials is also considered. The lumped- 


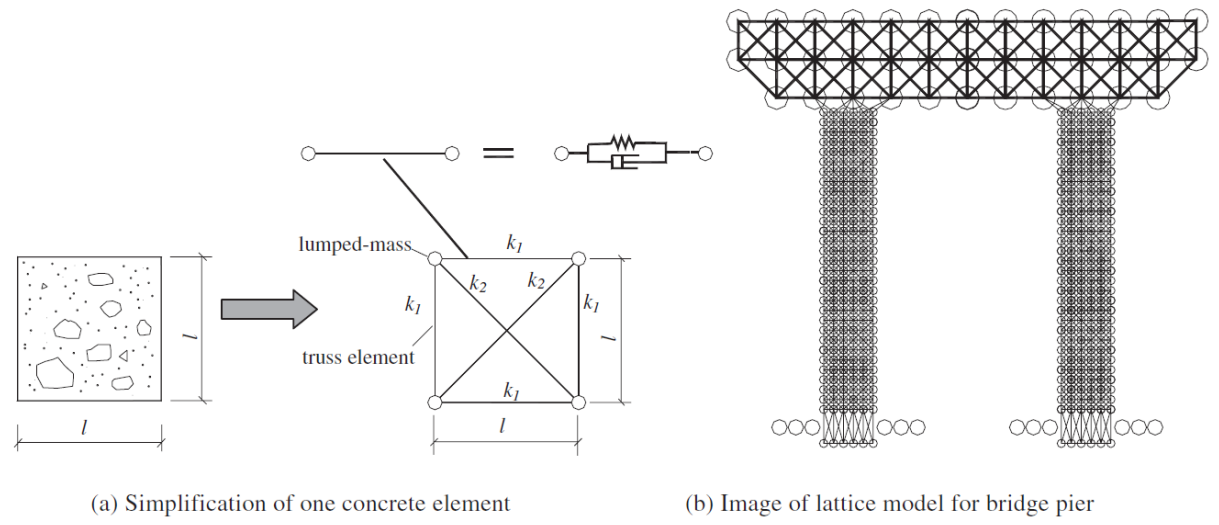

Figure 3.8: Concrete Lattice Model [41]

mass cannot be treated as an abstract point in this case, rather as a planar circular solid with a some radius after fracture of all connected springs [41]. Each two-dimensional square element is modelled as a lattice model cell composed of four lumped masses and six uni-axial concrete truss members. Every concrete truss member consists of a concrete spring and damper. The initial stiffness of the horizontal and vertical truss is $k_{1}$, and the two diagonal trusses is $k_{2}$. Under a unit compressive load the vertical and horizontal deformations of the concrete element are shown in Equations 3.82 and 3.83.

$$
\begin{aligned}
\delta_{1}^{C}=\frac{2 l}{E A} & =\frac{2 l}{E t l}=\frac{2}{E t} \\
\delta_{2}^{C} & =v \delta_{1}=\frac{2 v}{E t}
\end{aligned}
$$

where $E$ is the elastic modulus, and $v$ is the Poisson's ratio of concrete, $t, A$ and $l$ are the thickness, the sectional area and the length of the element. The deformations of the lattice model are;

$$
\begin{aligned}
& \delta_{1}^{L}=\frac{2\left(2 k_{1}+k_{2}\right)}{2 k_{1}\left(k_{1}+k_{2}\right)} \\
& \delta_{2}^{L}=\frac{k}{2 k_{1}\left(k_{1}+k_{2}\right)}
\end{aligned}
$$

Equating the deformations of the two systems gives;

$$
\delta_{1}^{C}=\delta_{1}^{L}, \quad \delta_{2}^{C}=\delta_{2}^{L}
$$


$k_{1}$ and $k_{2}$ are;

$$
\begin{array}{r}
k_{1}=\frac{1}{2(1+v)} E t \\
k_{2}=\frac{v}{1-v^{2}} E t
\end{array}
$$

Under a unit shear load, the shear deformations for the systems are;

$$
\begin{array}{r}
\delta_{3}^{C}=\frac{2}{G A / L}=\frac{2}{G t}=\frac{1+v}{E t} \\
\delta_{3}^{L}=\frac{2}{k_{2}}
\end{array}
$$

Equating 3.89 and 3.90, gives;

$$
k_{2}=\frac{E t}{2(1+v)}
$$

It must be noted that Equations 3.88 and 3.91 are satisfied simultaneously when $v=1 / 3$. For concrete, this value increases the limit of $0.1-0.2$. Therefore, Equation 3.88 should be used for a beam-like member, where the deformation is primarily a results of flexural strain. Equation 3.91 should be used for wall-like members in which the deformation is mainly due to the shear strain [41].

For modelling the steel bars in the concrete, the stiffness of the springs are shown in Equation 3.92. The steel bars are treated as lumped masses connected to one another by an axial spring.

$$
k_{s}=\frac{E_{s} A_{s}}{l_{s}}
$$

where, $E_{s}$ is the elastic modulus of the steel, $A_{s}$ is the cross-sectional area of the steel bar, and $l_{s}$ is the displacement between two adjacent nodes. The nodes of the steel bar and the concrete are independent of one another. The model can simulate local failure, and local buckling of the bar, and stripping of the concrete cover. The springs for bonding and sliding between steel and concrete are also considered. 


\subsection{Rigid Bodies Spring Models}

The Rigid Bodies Spring Models (RBSM), has several different formulations for different element types. For the scope of this project, the beam element and the plane strain elements are the most relevant, and are shown in the following sections.

\subsubsection{Formulation of Beam Element}

Consider the deformation of two rigid bars that are connected by one rotational spring in the middle, shown in Figure 3.9. At locations A,B, and C, the bars have a displacement of $u_{i-1}, u_{i}$ and $u_{i+1}$, and a force applied of $X_{i-1}, X_{i}$ and $X_{i+1}$, respectively. The strain energy of the bar is shown in Equation 3.93. where, $k_{b}$

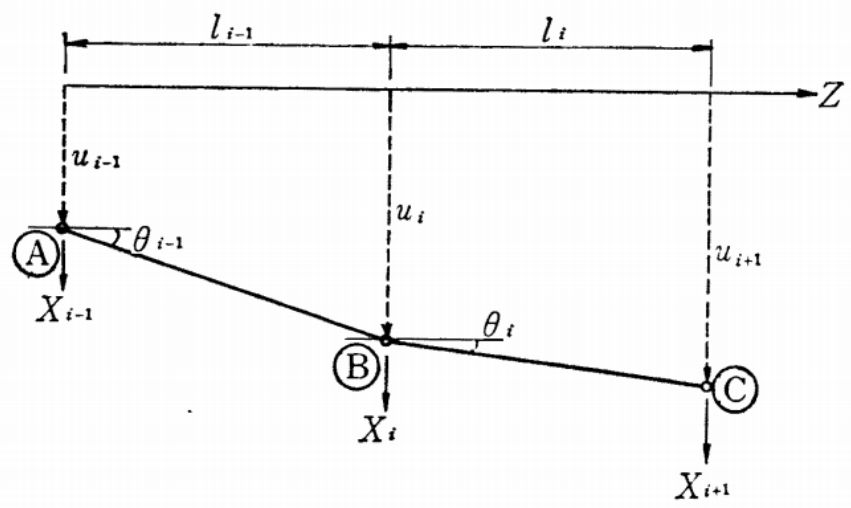

Figure 3.9: Beam Bending Element [43]

is the spring constant. Then it is written in terms of the displacements $u_{i-1}, u_{i}$ and $u_{i+1}$, Equation 3.94

$$
\begin{array}{r}
V\left(\theta_{i-1}, \theta_{i}\right)=\frac{k_{b}}{2}\left(\theta_{i}-\theta_{i-1}\right)^{2} \\
V\left(u_{i-1}, u_{i}, u_{i+1}\right)=\frac{k_{b}}{2}\left[\left(\frac{u_{i+1}-u_{i}}{l_{i}}\right)-\left(\frac{u_{i}-u_{i-1}}{l_{i-1}}\right)\right]^{2}
\end{array}
$$

The stiffness matrix can then be resolved by applying Castigliano's theorem on Equation 3.94, which leads to the stiffness matrix shown in Equation 3.101. The 
partial derivatives of the energy are as follows

$$
\begin{array}{r}
\frac{\partial V}{\partial u_{i-1}}=k\left(\frac{1}{l_{i-1}}\right)\left(\frac{u_{i+1}-u_{i}}{l_{i}}+\frac{-u_{i}+u_{i-1}}{l_{i-1}}\right) \\
\frac{\partial V}{\partial u_{i}}=k\left(\frac{-1}{l_{i}}-\frac{1}{l_{i-1}}\right)\left(\frac{u_{i+1}-u_{i}}{l_{i}}+\frac{-u_{i}+u_{i-1}}{l_{i-1}}\right) \\
\frac{\partial V}{\partial u_{i+1}}=k\left(\frac{1}{l_{i}}\right)\left(\frac{u_{i+1}-u_{i}}{l_{i}}+\frac{-u_{i}+u_{i-1}}{l_{i-1}}\right)
\end{array}
$$

Rewriting the equations in terms of $u_{i-1}, u_{i}$ and $u_{i+1}$, gives Equations $3.98-3.100$

$$
\begin{array}{r}
X_{i-1}=k\left[\left(\frac{1}{l_{i-1}^{2}}\right) u_{i-1}-\left(\frac{1}{l_{i}} \frac{1}{l_{i-1}}+\frac{1}{l_{i-1}^{2}}\right) u_{i}+\left(\frac{1}{l_{i-1}^{2}}\right) u_{i+1}\right] \\
X_{i}=k\left[-\left(\frac{1}{l_{i}} \frac{1}{l_{i-1}}+\frac{1}{l_{i-1}^{2}}\right) u_{i-1}+\left(\frac{1}{l_{i}}+\frac{1}{l_{i-1}}\right)^{2} u_{i}+\left(\frac{-1}{l_{i}}-\frac{1}{l_{i-1}}\right) u_{i+1}\right] \\
X_{i+1}=k\left[\left(\frac{1}{l_{i}} \frac{1}{l_{i-1}}\right) u_{i-1}-\left(\frac{1}{l_{i}} \frac{1}{l_{i-1}}+\frac{1}{l_{i}^{2}}\right) u_{i}+\left(\frac{1}{l_{i}^{2}}\right) u_{i+1}\right]
\end{array}
$$

Rewriting Equations 3.98 - 3.100 in matrix format, gives the stiffness matrix in Equation 3.101.

$$
\left(\begin{array}{c}
X_{i-1} \\
X_{i} \\
X_{i+1}
\end{array}\right)=k_{b}\left[\begin{array}{ccc}
\frac{1}{l_{i-1}^{2}} & & \text { sym } \\
\frac{-1}{l_{i-1}}\left(\frac{1}{l_{i}}+\frac{1}{l_{i-1}}\right) & \left(\frac{1}{l_{i}}+\frac{1}{l_{i-1}}\right)^{2} & \\
\frac{-1}{l_{i-1} l_{i}} & \frac{-1}{l_{i-1}}\left(\frac{1}{l_{i}}+\frac{1}{l_{i-1}}\right) & \frac{1}{l_{i}^{2}}
\end{array}\right]\left(\begin{array}{c}
u_{i-1} \\
u_{i} \\
u_{i+1}
\end{array}\right)
$$

\subsubsection{Plane strain elements}

Considering the beam bending model in plane strain, where two rigid triangular plates are connected by three different types of springs at the middle of the boundary edges, shown in Figure 3.10. Points 1 and 2 are the centroids of $\triangle A B C$ and $\triangle A C D . \triangle A B C$ and $\triangle A C D$ are connected at the middle of $\overline{A C}$ by three types of springs $k_{d}, k_{s}$ and $k_{r}$. The centroid displacements of the plate are denoted by $\left(u_{1}, v_{1}, \theta_{1}\right)$ and $\left(u_{2}, v_{2}, \theta_{2}\right)$.

The potential energy of the two elements is given in Equation 3.102:

$$
V=\frac{1}{2} k_{s}\left(u_{1}+u_{1}\right)^{2}+\frac{1}{2} k_{d}\left(v_{1}+v_{2}\right)^{2}
$$




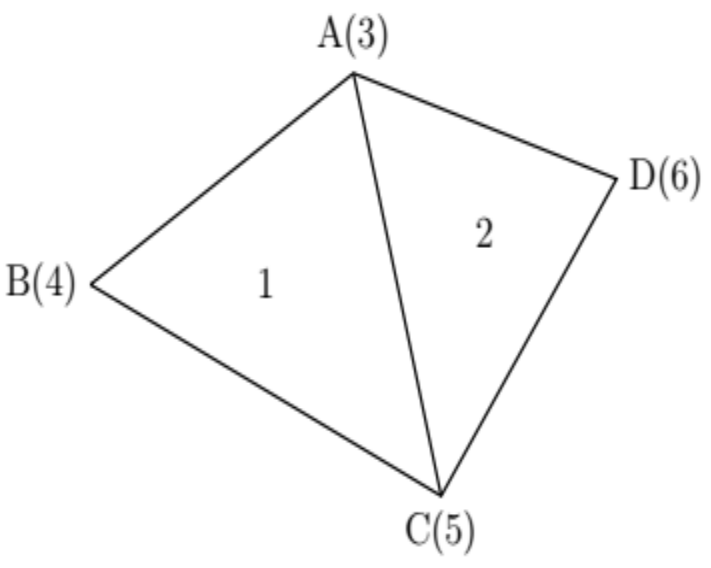

(a) RBSM 2 element body

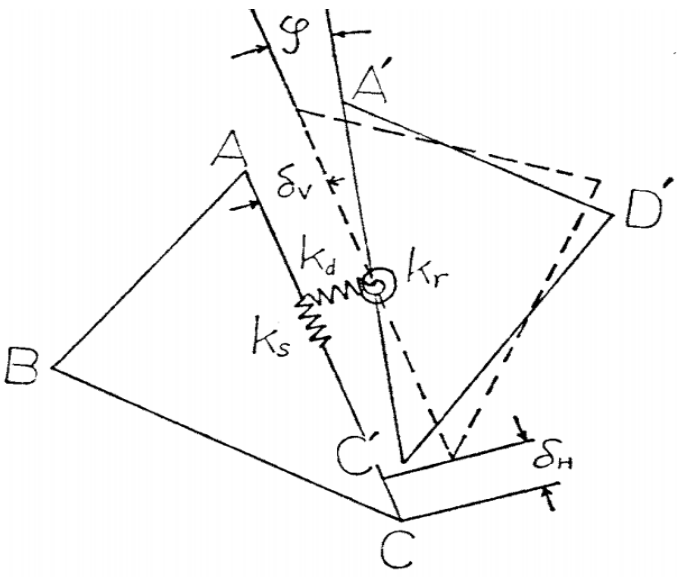

(b) RBSM deformed

Figure 3.10: Plane strain element in RBSM [43]

Applying Castigliano's theorem gives;

$$
\begin{array}{ll}
\frac{\partial V}{\partial u_{1}}=k_{s}\left(u_{1}+u_{2}\right), & \frac{\partial V}{\partial u_{2}}=k_{s}\left(u_{1}+u_{2}\right) \\
\frac{\partial V}{\partial v_{1}}=k_{d}\left(v_{1}+v_{2}\right), & \frac{\partial V}{\partial v_{2}}=k_{d}\left(v_{1}+v_{2}\right)
\end{array}
$$

Writing the equations in matrix format gives:

$$
\left[\begin{array}{cccc}
k_{s} & 0 & k_{s} & 0 \\
0 & k_{d} & 0 & k_{d} \\
k_{s} & 0 & k_{s} & 0 \\
0 & k_{d} & 0 & k_{d}
\end{array}\right]\left(\begin{array}{c}
u_{1} \\
v_{1} \\
u_{2} \\
v_{2}
\end{array}\right)
$$

Considering that the angle of rotation is shown in Figure 3.10 and is $\gamma$, and given that a transformation is matrix is given by $\left[\begin{array}{cc}\cos \theta & \sin \theta \\ -\sin \theta & \cos \theta\end{array}\right]$. Since $\theta=90-\gamma$, then the transformation matrix will be $\left[\begin{array}{cc}\sin \gamma & \cos \gamma \\ -\cos \gamma & \sin \gamma\end{array}\right]$. For $\operatorname{simplicity} \sin (\gamma)$ and $\cos (\gamma)$ will be denoted by $s$ and $c$ respectively. Transforming the stiffness matrix using $T^{\prime} K$, Equation 3.106: 


$$
\left[\begin{array}{cccc}
s & -c & 0 & 0 \\
c & s & 0 & 0 \\
0 & 0 & s & -c \\
0 & 0 & c & s
\end{array}\right]\left[\begin{array}{cccc}
k_{s} & 0 & k_{s} & 0 \\
0 & k_{d} & 0 & k_{d} \\
k_{s} & 0 & k_{s} & 0 \\
0 & k_{d} & 0 & k_{d}
\end{array}\right]=\left[\begin{array}{cccc}
k_{s} s & -k_{d} c & 0 & 0 \\
k_{s} c & k_{d} s & 0 & 0 \\
0 & 0 & k_{s} s & -k_{d} c \\
0 & 0 & k_{s} c & k_{d} s
\end{array}\right]
$$

now, $T^{\prime} K T$ gives the stiffness matrix shown in Equation 3.108.

$$
\begin{gathered}
{\left[\begin{array}{cccc}
k_{s} s & -k_{d} c & 0 & 0 \\
k_{s} c & k_{d} s & 0 & 0 \\
0 & 0 & k_{s} s & -k_{d} c \\
0 & 0 & k_{s} c & k_{d} s
\end{array}\right] \times\left[\begin{array}{cccc}
s & c & 0 & 0 \\
-c & s & 0 & 0 \\
0 & 0 & s & c \\
0 & 0 & -c & s
\end{array}\right]=} \\
{\left[\begin{array}{cccc}
k_{s} s^{2}+k_{d} c^{2} & k_{s} s c-k_{d} s c & 0 & 0 \\
k_{s} s c-k_{d} s c & k_{s} c^{2}+k_{d} s^{2} & 0 & 0 \\
0 & 0 & k_{s} s^{2}+k_{d} c^{2} & k_{s} s c-k_{d} s c \\
0 & 0 & k_{s} s c-k_{d} s c & k_{s} c^{2}+k_{d} s^{2}
\end{array}\right]}
\end{gathered}
$$

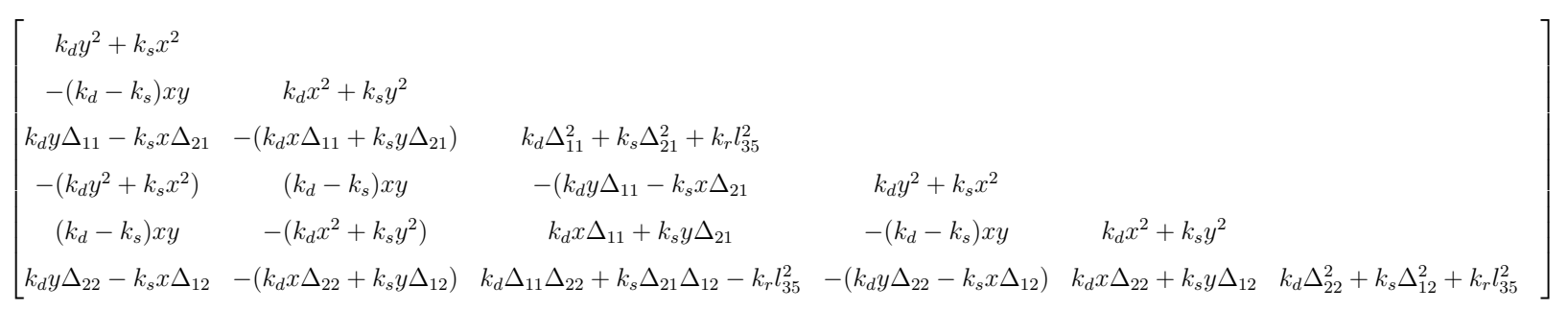

where $y=y_{53}$ and $x=x_{5} 3$.

Consider a set of $3 \mathrm{D}$ rigid bodies of arbitrary shape. They are assumed to be in equilibrium with external loads.

\subsection{Shifted Integration Technique Formulation}

There are two versions of the shifted integration technique; one utilising the Bernoulli-Euler beam elements, and the other using the linear Timoshenko beam elements. Since the application of the technique will be for modelling fracture 
and collapse, the Timoshenko beam element with one integration point is more suitable, because fracture cannot be considered by a single Bernoulli-Euler beam element [50].

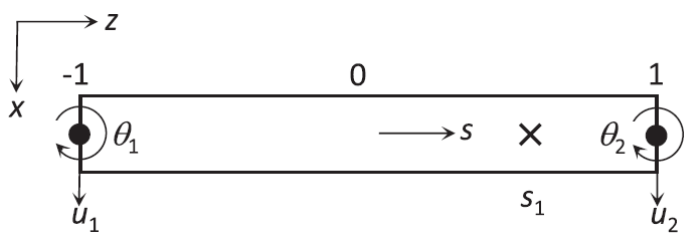

(a) Timoshenko beam element

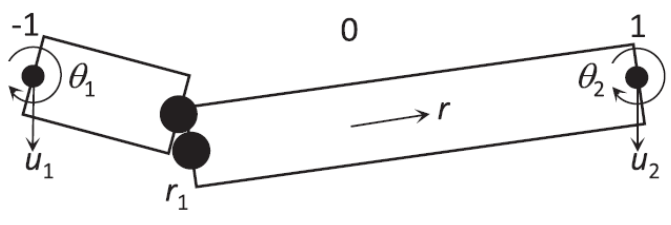

(b) Rigid Body Spring Model element

Figure 3.11: Timoshenko beam element compared to RBSM element [50]

\subsubsection{Adaptively Shifted Integration Technique}

The location of the numerical integration point during the elastic stage is the midpoint of the element. When a fully plastic sections occurs, the numerical integration point is shifted to the location at where the plastic hinge would occur. Figure 3.11 is a linear Timoshenko beam element and its equivalence to the RBSM. The location of the numerical integration point and stress evaluation point is shown in Equation 3.110 [47].

$$
s_{1}=-r_{1}
$$

When the entire region is elastic, $s=s_{1}=0$ and $r=r_{1}=0$, since the integration point is at midpoint. The stiffness matrix for the element is as follows;

$$
\left[K_{E}\right]=l[B(0)]^{T}\left[D_{e}(0)\right][B(0)]
$$

The strain vector $\left.\{\Delta)_{n} \varepsilon(0)\right\}$ and the force increment vector $\left.\{\Delta)_{n} \sigma(0)\right\}$ at $r=$ $r_{1}=0$ are;

$$
\begin{array}{r}
\left\{\Delta_{n} \varepsilon(0)\right\}=[B(0)]\left\{\Delta_{n} u\right\} \\
\left\{\Delta_{n} \sigma(0)\right\}=\left[D_{e}(0)\right]\left\{\Delta_{n} \varepsilon(0)\right\}
\end{array}
$$


The forces calculated in Equation 3.113 are at the midpoint of the element. The bending moments along the elastic deformed element are;

$$
\begin{aligned}
& \Delta_{n} M_{x}(s)=\Delta_{n} M_{x}(0)-\Delta_{n} V_{y}(0) \frac{l_{s}}{2} \\
& \Delta_{n} M_{y}(s)=\Delta_{n} M_{y}(0)-\Delta_{n} V_{x}(0) \frac{l_{s}}{2}
\end{aligned}
$$

Once a plastic section has fully formed, the integration points are shifted to $s=s_{1}=-r_{1}$. The elemental stiffness matrix, the generalized strain and the force increment vectors are as follows;

$$
\begin{array}{r}
{\left[K_{E}\right]=l\left[B\left(s_{1}\right)\right]^{T}\left[D_{p}\left(r_{1}\right)\right]\left[B\left(s_{1}\right)\right]} \\
\left\{\Delta_{n} \varepsilon\left(r_{1}\right)\right\}=\left[B\left(s_{1}\right)\right]\left\{\Delta_{n} u\right\} \\
\left\{\Delta_{n} \sigma\left(r_{1}\right\}=\left[D_{p}\left(r_{1}\right)\right]\left\{\Delta_{n} \varepsilon\left(r_{1}\right)\right\}\right.
\end{array}
$$

where $\left[D_{p}(r)\right]$ is the force-strain matrix for plastic deformation. This matrix can be expressed as;

$$
\left[D_{p}\left(r_{1}\right)\right]=\left[D_{e}\left(r_{1}\right)\right]-\frac{\left[D_{e}\left(r_{1}\right)\right]\{\partial f / \partial \sigma\}[\partial f / \partial \sigma]\left[D_{e}\left(r_{1}\right)\right]}{H^{\prime}+[\partial f / \partial \sigma]\left[D_{e}\left(r_{1}\right)\right]\{\partial f / \partial \sigma\}}
$$

where, $H^{\prime}$ is the strain hardening coefficient and $f$ is the plastic potential expressed as;

$$
f=f_{y}\left(\sigma\left(r_{1}\right)\right)-1=0
$$

\subsubsection{ASI-Gauss Technique}

In both the ASI and ASI-Gauss techniques, the numerical integration point is shifted adaptively if a full plastic section is developed to express a plastic hinge. When the plastic hinge is unloaded the numerical integration point is shifted back to its initial locations. The initial location of the integration point in the ASI is the midpoint of the beam element, and is considered optimal when the entire element is elastic. Bending deformations are inaccurate because the displacement functions of the element are defined by linear functions. An efficient way to solve 
the discrepancy is using the ASI-Guass technique where the numerical integration points of a member coincide with the Gaussian integration points of the member, shown in Figure 3.12. The locations are optimal for two-point integration in Gaussian quadrature and the accuracy of the deformation defined by a cubic function is guaranteed. The relation between the locations of the numerical inte-

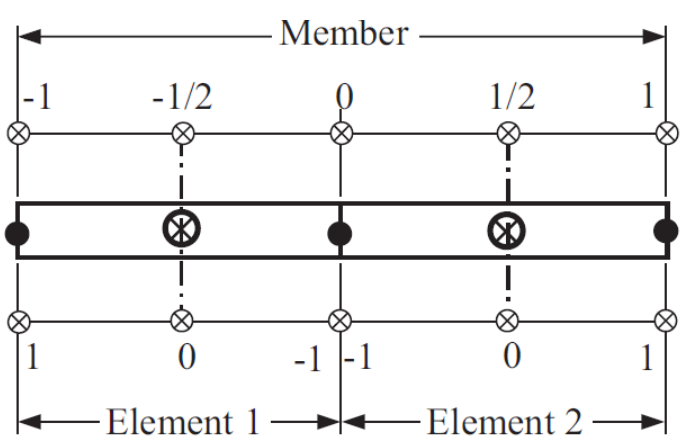

(a) ASI

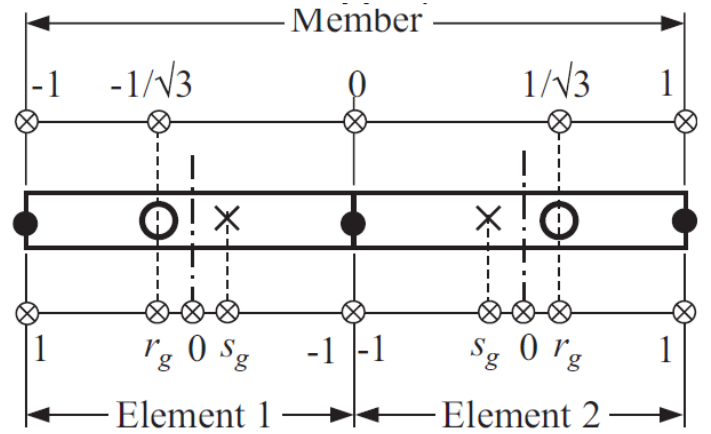

(b) ASI-Gauss

Figure 3.12: ASI element compared to ASI-Gauss [50]

gration point and the stress evaluation point is the same as Equation 3.110 [47]. The numerical integration points of the elements in the ASI-Gauss technique are places at $s=s_{1}=s_{g}$ when the element is entirely elastic. The initial locations of the numerical integration points in the ASI-Gauss technique is $s_{g}=1-(2 / \sqrt{(3)})$, and the stress evaluation points is $r_{g}=-s_{g}=-1+(2 / \sqrt{(3)})$. Therefore, the elemental stiffness matrix, the strain and the force increment vectors in the ASIGauss technique are shown in Equations 3.121-3.123.

$$
\begin{array}{r}
{\left[K_{E}\right]=l\left[B\left(s_{g}\right)\right]^{T}\left[D_{e}\left(r_{g}\right)\right]\left[B\left(s_{1} g\right)\right]} \\
\left\{\Delta_{n} \varepsilon\left(r_{g}\right)\right\}=\left[B\left(s_{g}\right)\right]\left\{\Delta_{n} u\right\} \\
\left\{\Delta_{n} \sigma\left(r_{e}\right\}=\left[D_{e}\left(r_{g}\right)\right]\left\{\Delta_{n} \varepsilon\left(r_{g}\right)\right\}\right.
\end{array}
$$

The bending moments are;

$$
\begin{aligned}
& \Delta_{n} M_{x}(s)=\Delta_{n} M_{x}\left(s_{g}\right)-\Delta_{n} V_{y}\left(s_{g}\right) \frac{l}{2}\left(s+s_{g}\right) \\
& \Delta_{n} M_{y}(s)=\Delta_{n} M_{y}\left(s_{g}\right)-\Delta_{n} V_{x}\left(s_{g}\right) \frac{l}{2}\left(s+s_{g}\right)
\end{aligned}
$$


The yield condition by the plastic flow theory is;

$$
f=\left(\frac{M_{x}}{M_{x o}}\right)^{2}+\left(\frac{M_{y}}{M_{y o}}\right)^{2}+\left(\frac{N}{N_{0}}\right)^{2}-1=f_{y}-1=0
$$

where $f_{y}$ is the yield function, and $M_{x}, M_{y}$ and $N$ are the bending moments around the x-axis, $\mathrm{y}$-axis and axial force respectively. The remainder of the equations are the same as the ASI technique. By adaptively shifting the numerical integration point of an element, a precise location of a plastic hinge is found and highly accurate elasto-plastic solutions can be obtained with a very small number of elements [50]. 


\section{Part II}

\section{Formulation of Linear and}

Nonlinear Gaussian Based AEM 


\section{Chapter 4}

\section{Applied Element Method Formulation}

To model the progressive collapse of structures the continuum and discrete element behaviour must be considered to capture all stages of collapse. In Chapters 2 and 3 , different methods were presented along with their formulations to present the brief progression of methods used for modelling the progressive collapse. Out of all the methods that were presented, the Applied Element Method stood out, since it is based on combining the FEM and DEM in some sense. Since the AEM was developed to aid in the analysis of highly nonlinear behaviour of structures, it is selected as the appropriate model to analyse the progressive collapse of structures.

In this chapter a complete formulation of the AEM is presented along with modifications to the method that have been presented in the previous decades for use in different applications.

\subsection{AEM Formulation for 2-D element}

The AEM was developed in year 2000 by Meguro and Tagel-Din [6]. The elements in AEM are rigid bodies that are connected with sets of normal and shear springs along the edges of the elements. The springs represent the two connecting elements in that region. The material properties are specified through the spring stiffness. For a 2-D element, three degrees-of-freedom are considered per element: 
deflection in $\mathrm{x}$, deflection in $\mathrm{y}$ and rotation [6].

Consider a linear spring that is subjected to axial forces at both ends of the spring, $f_{1}$ and $f_{2}$. The displacement at each end of the spring is represented as $u_{1}$ and $u_{2}$. Assuming the spring is in equilibrium then the sum of the forces should be zero. So, $f_{1}+f_{2}=0$, or $f_{1}=-f_{2}$. By using the spring constant $k$, the force displacement relationship of each spring from Hooke's law gives, $k\left(u_{1}-u_{2}\right)=f_{1}$ and $k\left(u_{2}-u_{1}\right)=f_{2}$. Rewriting these equations in matrix form gives

$$
\left[\begin{array}{cc}
k & -k \\
-k & k
\end{array}\right]\left\{\begin{array}{l}
u_{1} \\
u_{2}
\end{array}\right\}=\left\{\begin{array}{l}
f_{1} \\
f_{2}
\end{array}\right\}
$$

The equivalent spring constant for a linear spring when considered as an axial member with cross-sectional area $A$ and length $l$ is shown in Equation 4.2.

$$
k=\frac{E A}{l}
$$

The stiffness matrix for a pair of elements is a $6 \times 6$ matrix shown in Equation 4.3. The upper left quadrant of the matrix is displayed in Equation 4.4 [6]. Each spring location in an element is represented by a pair of normal and shear springs, with stiffness displayed in Equation 4.5. The elements distribution is displayed in Figure 4.1, and the details in elements are presented in Figure 4.2.

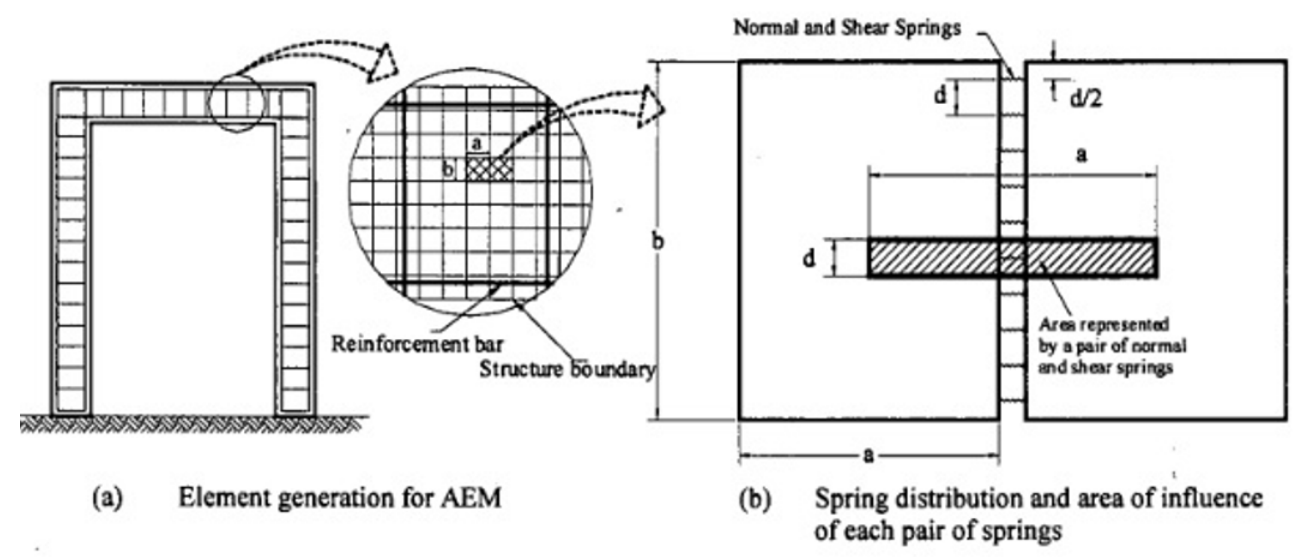

Figure 4.1: 2D AEM Elements [6]

$$
K=\left[\begin{array}{ll}
k_{11} & k_{12} \\
k_{21} & k_{22}
\end{array}\right]
$$




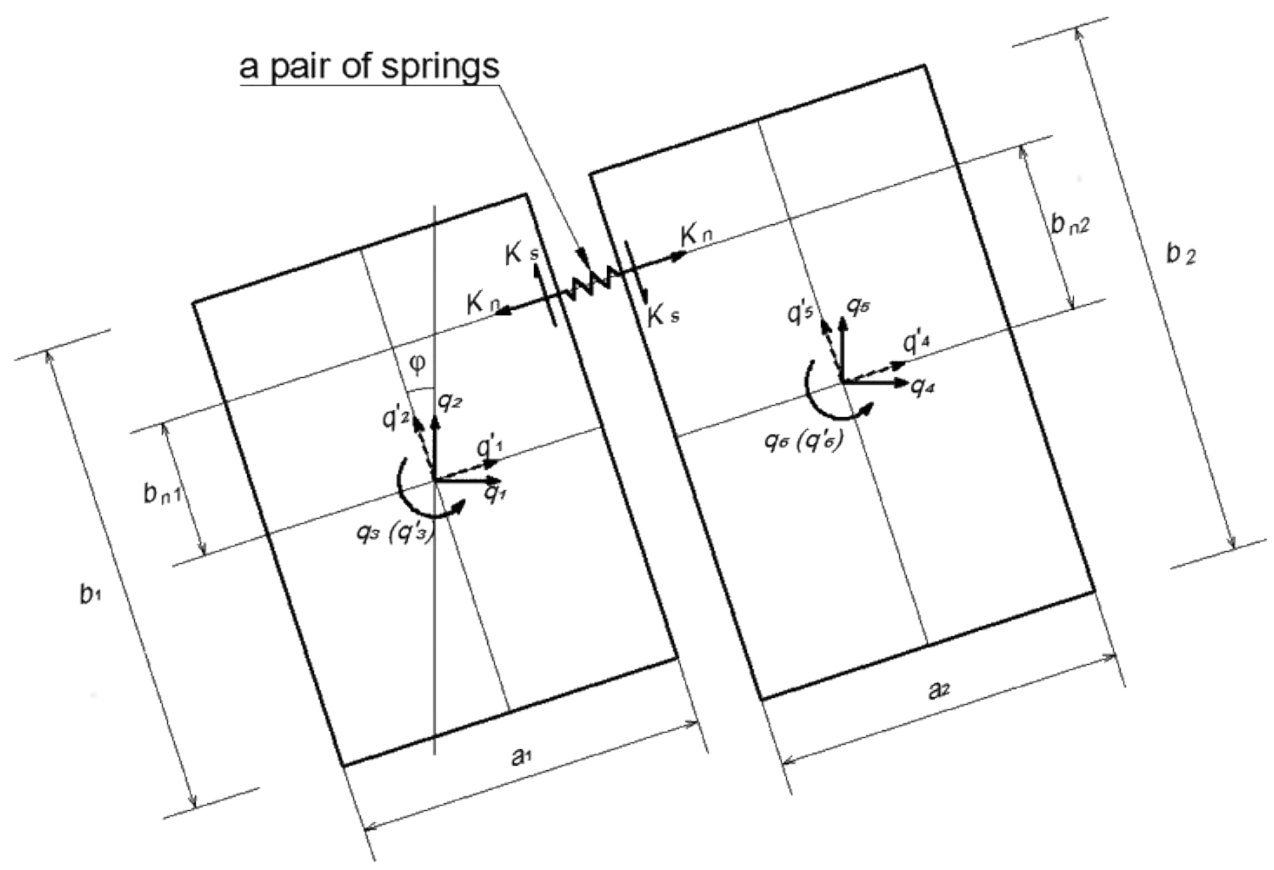

Figure 4.2: 2D AEM Elements degrees-of-freedom [65]

where $k_{11}$ is

$$
k_{11}=\left[\begin{array}{ccc}
K_{n 1} & 0 & -K_{n 1} b_{n 1} \\
0 & K_{s 1} & K_{s 1} \frac{a}{2} \\
-K_{n 1} b_{n 1} & K_{s 1} \frac{a}{2} & \left(K_{n 1} b_{n 1}\right)+\left(K_{s 1} \frac{a}{4}\right)
\end{array}\right]
$$

where $K_{n}$ and $K_{s}$ are

$$
K_{n}=\frac{E d t}{a} ; \quad K_{s}=\frac{G d T}{a}
$$

where $E$ is the Young's Modulus of elasticity of the material; $G$ is the shear modulus of elasticity of the material; $b_{n 1}$ is the distance between the centre of the element and the spring location, that can be positive, negative or zero; $d$ is the length from the tributary area each spring covers; $a$ is the width from the tributary area each spring covers (also the width of the element if the elements are the same size, since the width of the element is also the width from centre to centre of two adjacent elements); and $t$ is the thickness of the element. $K$ is the stiffness matrix in the local system, represented by $q$ in Figure 4.2. The local to global transformation matrix $L$, Equation 4.6 is used to calculate the stiffness 
matrix in the global system.

$$
L=\left[\begin{array}{cccccc}
c & s & 0 & 0 & 0 & 0 \\
-s & c & 0 & 0 & 0 & 0 \\
0 & 0 & 1 & 0 & 0 & 0 \\
0 & 0 & 0 & c & s & 0 \\
0 & 0 & 0 & -s & c & 0 \\
0 & 0 & 0 & 0 & 0 & 1
\end{array}\right]
$$

where $c=\cos (\varphi)$ and $s=\sin (\varphi) . \quad \varphi$ is shown in Figure 4.2, and is the angle between the local q axis, and the global y axis. The transformation of the local $K$ matrix to the global $K^{\text {global }}$ matrix for one element is shown in Equation 4.7.

$$
K^{\text {global }}=L^{T} K L
$$

\subsection{AEM Meshing and Element distribution}

MATLAB is used to mesh beam structural elements. Consider a cantilever beam displayed in Figure 4.3. As mentioned previously the AEM elements are rectangular, so distributing elements is straightforward in rectangular and square shaped structures. Figure 4.3 shows the mesh distribution for different number of elements.

8 nodes - x-direction

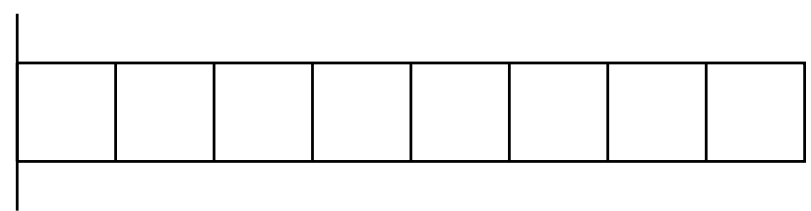

8 nodes - x-direction

2 nodes - y-direction

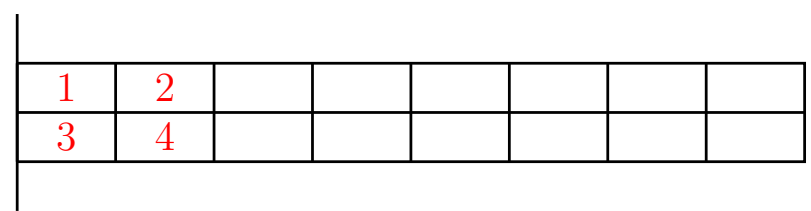

8 nodes - $\mathrm{x}$-direction

3 nodes - y-direction

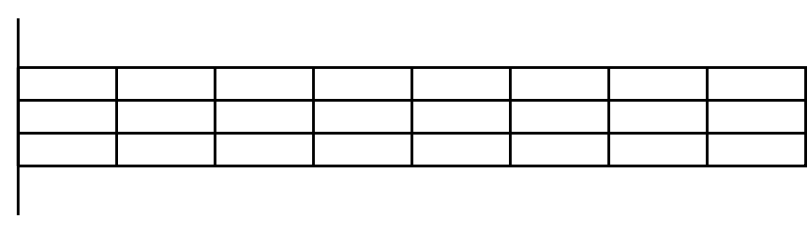

16 nodes - $\mathrm{x}$-direction

3 nodes - y-direction

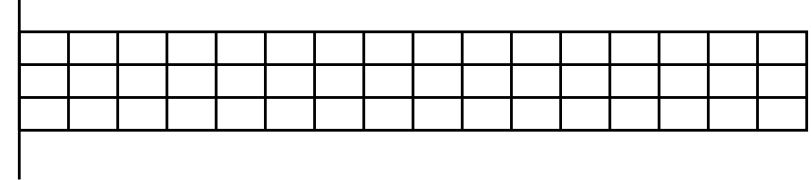


Figure 4.3: Mesh distribution for structural beam

In the second mesh for the beam configuration, with 8 elements in the $\mathrm{x}$-direction and 2 in the $y$-direction, the connectivity of springs between elements 1,2,3 and 4 are presented in Figure 4.4c, where all edges are connected by springs. Figure 4.4a displays the connectivity between elements 1 and 2, and Figure 4.4b shows the connectivity between elements 1 and 3. For a structural frame shown in Figure

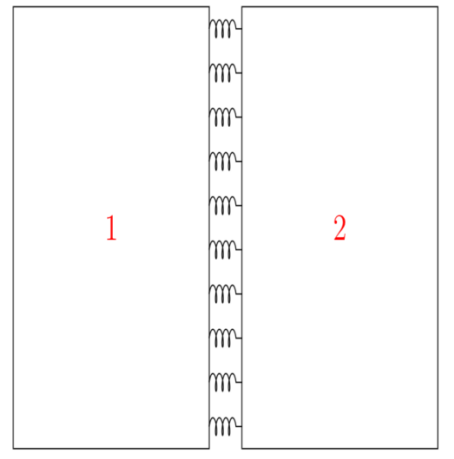

(a) Element 1-2

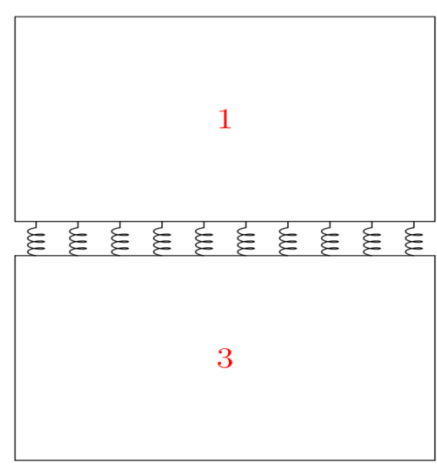

(b) Element 1-3

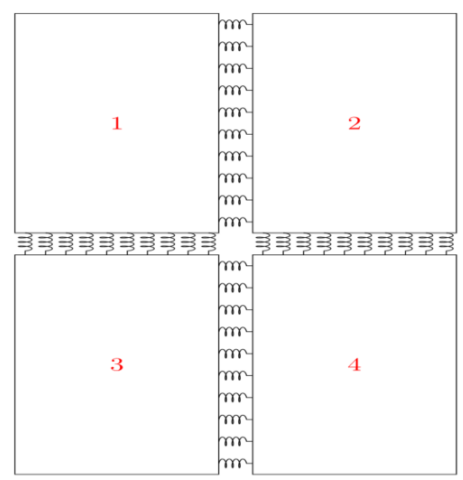

(c) Element 1-2-3-4

Figure 4.4: Connectivity between adjacent elements

4.5 the general meshing is shown, where the elements are connected by springs through the faces of the elements.

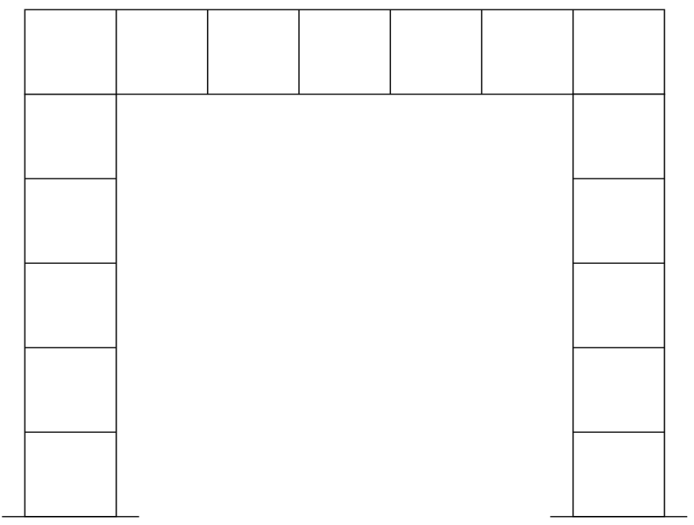

(a)

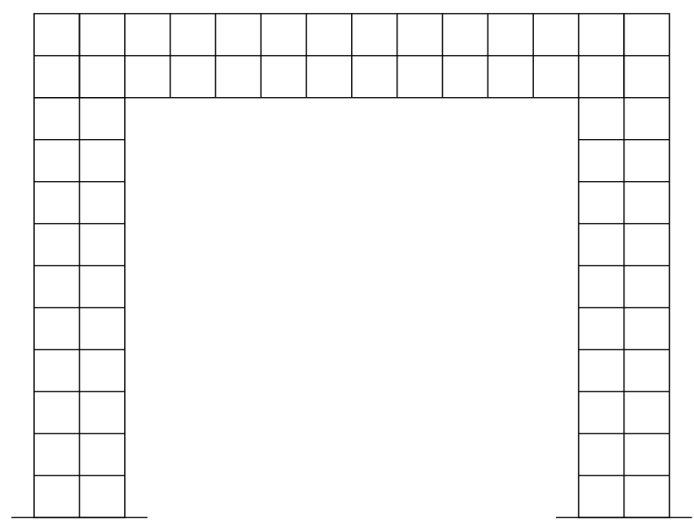

(b)

Figure 4.5: Frame meshing in AEM

\subsubsection{Structures with multiple materials}

One of the advantages of using springs between elements is that different material properties can be easily represented in different springs. For example, consider 
modelling a reinforced concrete beam. The springs that are depicting the concrete behaviour will have the modulus of elasticity of concrete, while the springs modelling the reinforcement will have the steel modulus of elasticity. Figure 4.6 displays an example of a beam with both concrete springs (represented in black) and reinforcing steel springs (represented in red). The highlighted red area depicts the location of the steel reinforcement, since that is the tributary area of all springs. Implementing this in the MATLAB code is done by defining which spring from the element has the reinforcement properties, and the analysis will be carried on. An example of this is displayed below.

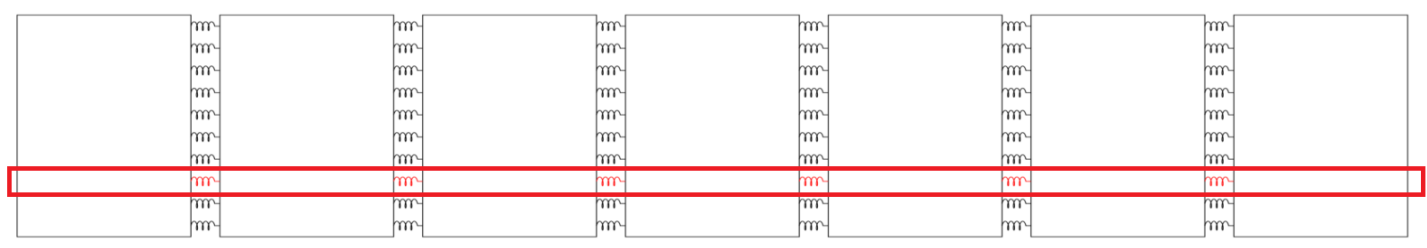

Figure 4.6: Representation of a cantilever concrete and steel reinforcement springs

In order to analyse a structural beam in the AEM code, the following input information is required:

\section{- Material properties}

- Beam geometry: span, width of cross-section, thickness of beam.

- AEM properties: Number of elements in the x-direction, number of elements in the y-direction, local dimensions of element ( $a$ and b), number of springs.

- Connectivity table: node 1 , node 2 , element orientation.

\subsection{Effect of Number of Springs and Elements}

Using different numbers of elements and springs when modelling structural elements will lead to different accuracy in results. This section provides the effect of the number of springs and number of elements in a system, and how increasing the accuracy of an analysis is achieved by changing these two variables. 


\subsubsection{Effect of Number of Springs Between Elements}

Firstly, the number of springs between elements is considered. In elastic analysis the number of springs may not seem as crucial as in nonlinear analysis, since increasing the number of springs between elements leads to better analysis of crack propagation. It is assumed that " $2 \mathrm{n}$ " evenly-spaced springs are connecting two elements together, where each spring represents the distance of " $d=b / 2 n$ " [6]. For translational degrees of freedom, number of connecting springs does not affect the element stiffness, since decreasing the number of springs increases the area represented by each spring, in the translational degrees-of-freedom case [6]. Theoretical rotational stiffness $K_{r}$, calculated from normal springs, is given in Equation $4.8[6]$.

$$
K_{r}=\int_{y=-b / 2}^{y=b / 2} \frac{E t}{b} \times b_{n 1} \times b_{n 1} \times d b_{n 1}=\frac{E t}{3 b} \times 2\left(\frac{b}{2}\right)^{3}=\frac{E t b^{2}}{12}
$$

The element rotational stiffness is obtained by summing up all the rotational stiffness calculates for each spring separately. Therefore, the total rotational stiffness is given in Equation 4.9. From Equation 4.8 substitute $\mathrm{b}=\mathrm{b} / 2 \mathrm{n}$, since b will now represent each spring separately. Therefore $\frac{E T b^{2}}{12}$ will be $\frac{E T b^{2}}{4 n^{3}}$ and is multiplied by the number of springs.

$$
K_{r}=\frac{E t b^{2}}{4 n^{3}} \sum_{i=1}^{n}(i-0.5)^{2}
$$

where $i$ is the spring number. The calculated rotational stiffness is a function of the number of connecting springs. From Reference [6], it was found that the rotational stiffness is smaller than the theoretical value by $25 \%$, and the error reduced to less than $1 \%$ when the number of connecting springs is larger than 10 . If the element size is relatively large in comparison to the structure size then the rotational stiffness affects the accuracy. However if the element size is small then the rotational stiffness cannot affect the system because the rotation between elements becomes small [6]. 


\subsubsection{Effect of Element Size}

The element size in the model is an important factor in the accuracy of results [6]. Large elements increase the structure stiffness and thus the failure load of the structure. A series of simulations were carried out of a laterally loaded cantilever to show the element size effect. Two analyses were performed using 10 and 20 springs respectively [6]. Figure 4.7a displays the cantilever with different numbers of base elements. Figure 4.7b shows the percentage error and CPU time for each cantilever with 10 and 20 springs. The results show that there is an increase of the CPU time as the number of elements increases, which is expected. Moreover, the ratio of error decreases as the number of elements increases. Using 10 springs resulted in half the CPU time of using 20 springs, but both spring cases resulted in the same ratio of error [6]. It is concluded that using a large number of elements with a relatively low number of connecting springs leads to a high accuracy with acceptable CPU time. Therefore, in order to increase the accuracy of an analysis, it is recommended to increase the number of elements rather than increasing the number of springs [6].

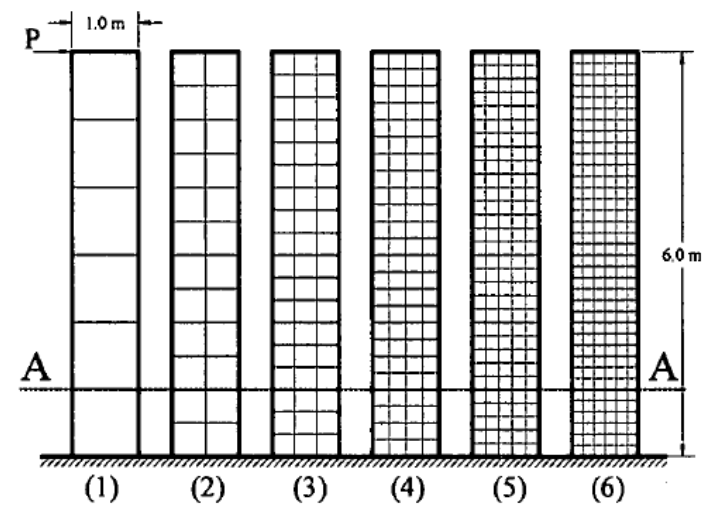

(a) Cantilever beams with different number of base elements

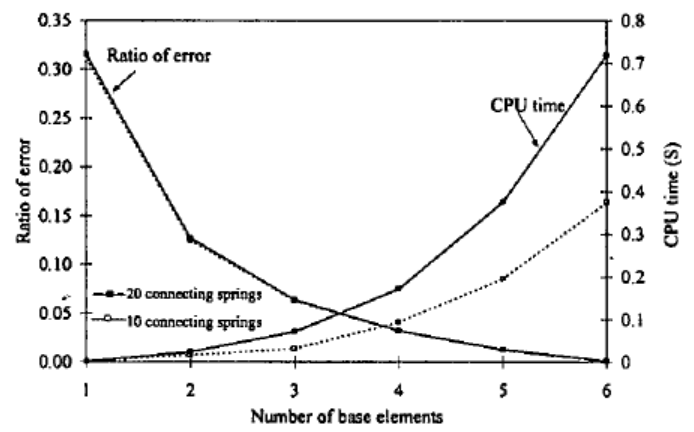

(b) CPU time and error difference

Figure 4.7: Effect of Element Size [6]

\subsection{Large Displacement Analysis using AEM}

The numerical procedure for simulating large displacement is shown below [66]. The modification to the displacement calculation equation is shown in Equation 
4.10.

$$
K \Delta \mathbf{u}=\Delta \mathbf{f}+\mathbf{R}_{\mathbf{m}}+\mathbf{R}_{\mathbf{G}}
$$

where $K$ is the linearised stiffness matrix; $\Delta \mathbf{u}$ is the incremental displacement vector; $\Delta \mathbf{f}$ is the incremental load vector; $\mathbf{R}_{\mathbf{m}}$ is the residual force vector due to cracking or incompatibility between spring strains and stresses; and $\mathbf{R}_{\mathbf{G}}$ is the residual force vector due to the geometrical changes in the structure during loading. The limitations of the method are as follows: (1) complete symmetry of the structure and loading must be avoided in buckling analysis; (2) small displacement theory is assumed during each increment, therefore small increments of load and displacements should be assumed; and (3) the apparent structure stiffness decreases after buckling.

\section{Box. 4.4.1: Large displacement analysis procedure in AEM}

1. Assume that $\mathbf{R}_{\mathbf{m}}$ and $\mathbf{R}_{\mathbf{G}}$ are null and solve Equation 4.10 to get $\Delta \mathbf{u}$.

2. Modify the structural geometry according to the calculated incremental displacements.

3. Modify the direction of the spring force vectors according to the new element configuration. The geometrical changes generate incompatibility between the applied forces and internal stresses.

4. Verify whether cracking occurred and calculate $\mathbf{R}_{\mathbf{m}}$. In elastic analysis $\mathbf{R}_{\mathbf{m}}=0$.

5. Calculate the element force vector, $F_{m}$, by summing the forces of the springs around each element.

6. Calculate the geometrical residuals around each element with Equation 4.11 .

$$
\mathbf{R}_{\mathrm{G}}=\mathbf{f}-\mathbf{F}_{\mathbf{m}}
$$

7. Small deformations are assumed during each increment.

8. Calculate the stiffness matrix for the structure with the new configuration considering stiffness changes due to cracking or yielding.

9. Repeat the entire process.

A simply supported beam was analysed to test the accuracy of the modifi- 
cation for large displacement [66]. The beam has a span of $12 m$, square cross section of $1 m$, and simply supported (pinned on one side and roller on the other), with a point load at the midspan. The Young's modulus is $210 \mathrm{MPa}$. Figure 4.8a displays the deflection of the beam without accounting for the modifications for the large displacement analysis, while Figure $4.8 \mathrm{~b}$ is the beam deflection with large displacement modification considered. In the case of small displacement analysis, the volume of the beam increases when the analysis approaches very large deformations, and the roller does not move, which is unrealistic. By using the large displacement theory, the more realistic results were found.

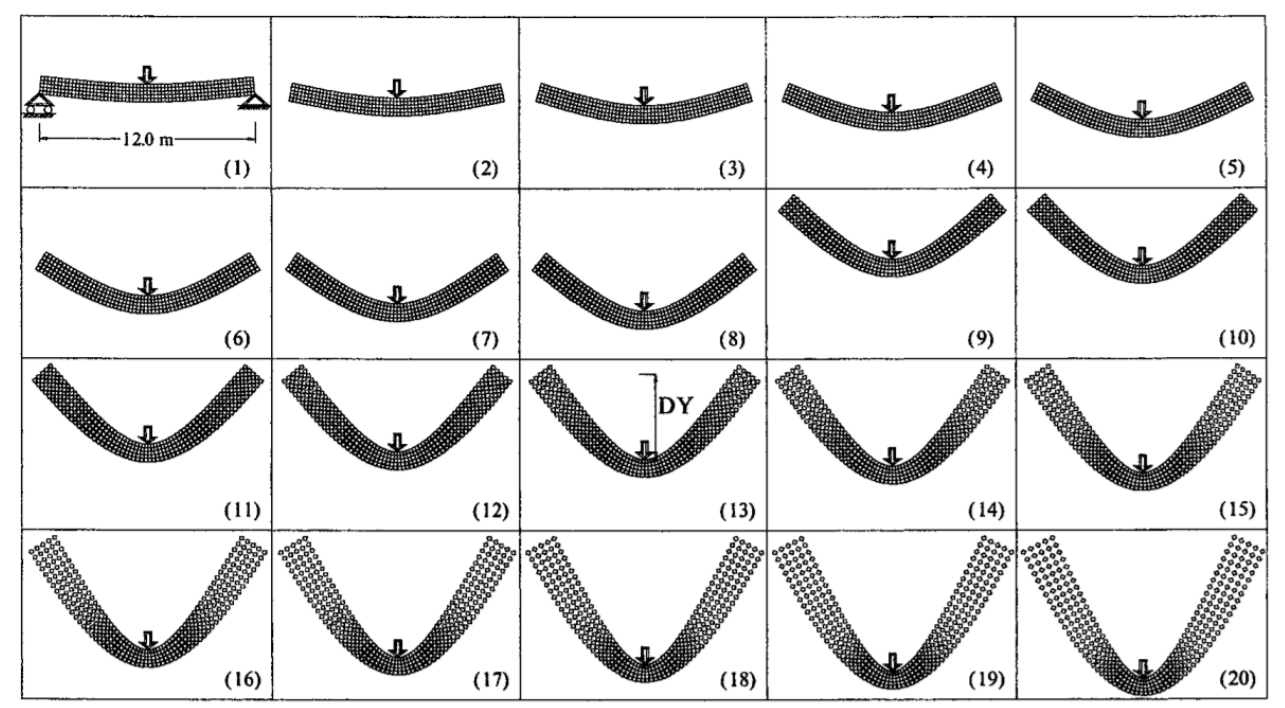

(a) Without large displacement modifications

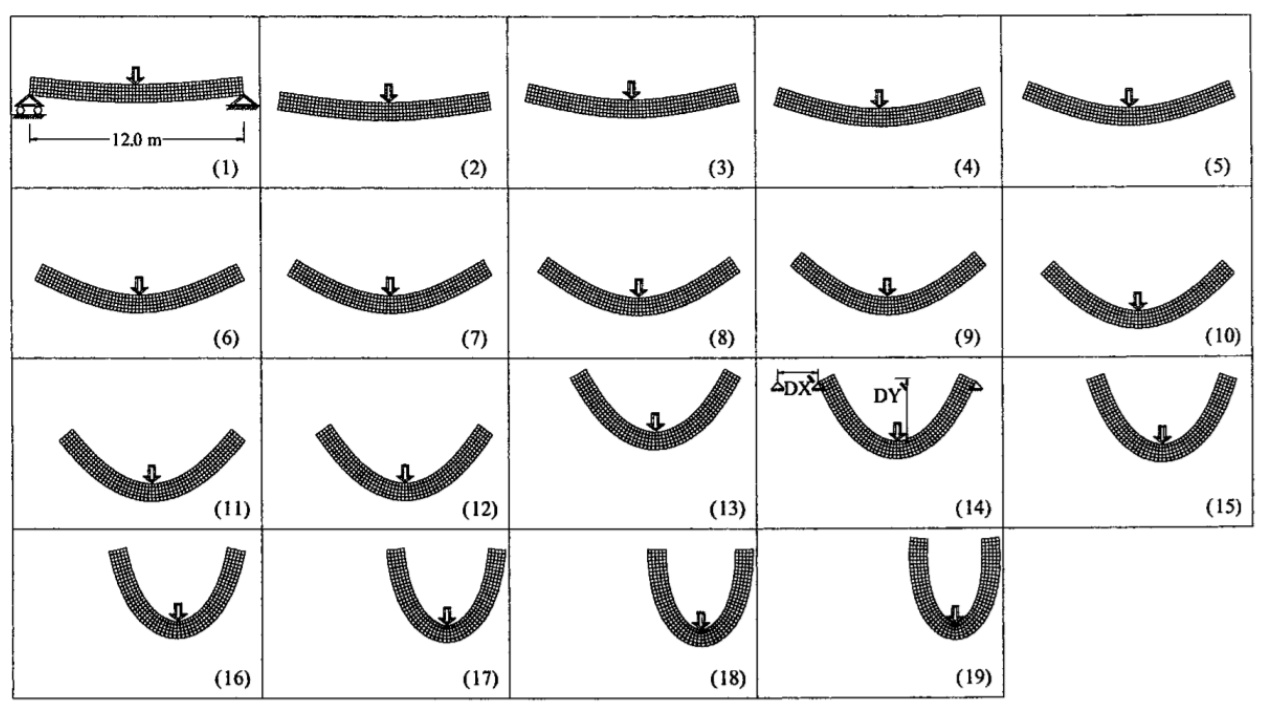

(b) With large displacement modifications

Figure 4.8: Large Displacement of Simply supported beam [66] 


\subsection{Modifications in AEM}

As mentioned in the previous chapter, some modifications have been done to the original AEM to improve the methods applicability. The modifications are the Improved Applied Element Method in 2003, and the Voronoi Applied Element method, 2008.

\subsubsection{Improved Applied Element Method}

The Improved Applied Element Method can accurately simulate the shear stiffness without using a large number of springs [58]. The normal and shear stiffness for each spring is shown in Equation 4.12.

$$
K_{N}^{i}=\frac{E d t_{N}^{i}}{a} \quad K_{S}^{i}=\frac{G d t_{S}^{i}}{a}
$$

where $t_{N}^{i}$ and $t_{s}^{i}$ are the thickness represented by spring $i$ for normal and shear cases respectively. The differences in the values of $t$ for each spring are due to the change of effective area for both normal and shear directions. This modification allows modelling different flanged steel sections, like I-beam, Box and Channel cross-sections. There were also modifications in the dynamic properties. Recalling that the general dynamic equation of motion governing the response of structure in small displacement is shown in Equation 4.13.

$$
[M]\{\Delta \ddot{\mathbf{u}}\}+[C]\{\Delta \dot{\mathbf{u}}\}+[K]\{\Delta \mathbf{u}\}=\Delta \mathbf{f}(t)-[M] \Delta\left\{\ddot{\mathbf{u}}_{\mathrm{G}}\right\}
$$

where $[M]$ is the mass matrix; $[C]$ is the damping matrix and $[K]$ is the nonlinear stiffness matrix; $\Delta \mathbf{f}(t)$ is the incremental applied load vector; $\{\Delta \ddot{\mathbf{u}}\},\{\Delta \dot{\mathbf{u}}\}$, $\{\Delta \mathbf{u}\}$ and $\left\{\Delta \ddot{\mathbf{u}}_{\mathbf{G}}\right\}$ are the incremental acceleration, velocity, displacement and gravity acceleration vectors, respectively. The mass matrix and the polar moment of inertia of each element are lumped at the element centroid. Equation 4.14 represents the value of lumped mass in each degree of freedom direction assuming 
elements having square shape [58].

$$
\left[\begin{array}{c}
M 1 \\
M 2 \\
M 3
\end{array}\right]=\left[\begin{array}{c}
D^{2} \cdot \rho \cdot t_{a v} \\
D^{2} \cdot \rho \cdot t_{a v} \\
\frac{D^{4} \cdot \rho}{n s p} \cdot \sum_{i=1}^{i=n s p}\left(\frac{t_{i}^{x}}{12}+\frac{t_{i}^{y}}{12}\right)
\end{array}\right]
$$

where $D$ is the element size; $t_{a v}$ is the average thickness of the element; $\rho$ is the density of the material. [M1] and [M2] correspond to the element mass, and $[M 3]$ is the element polar moment of inertia about the centre of gravity.

\subsubsection{Voronoi AEM}

The Voronoi AEM (VAEM) was presented by Worakanchana and Meguro to eliminate the disadvantages of using square shaped elements from the conventional AEM [59]. The element formulation of the VAEM is shown in Figure 4.9, and is based on the Voronoi tessellation [67]. Considering a two-particle assembly, each
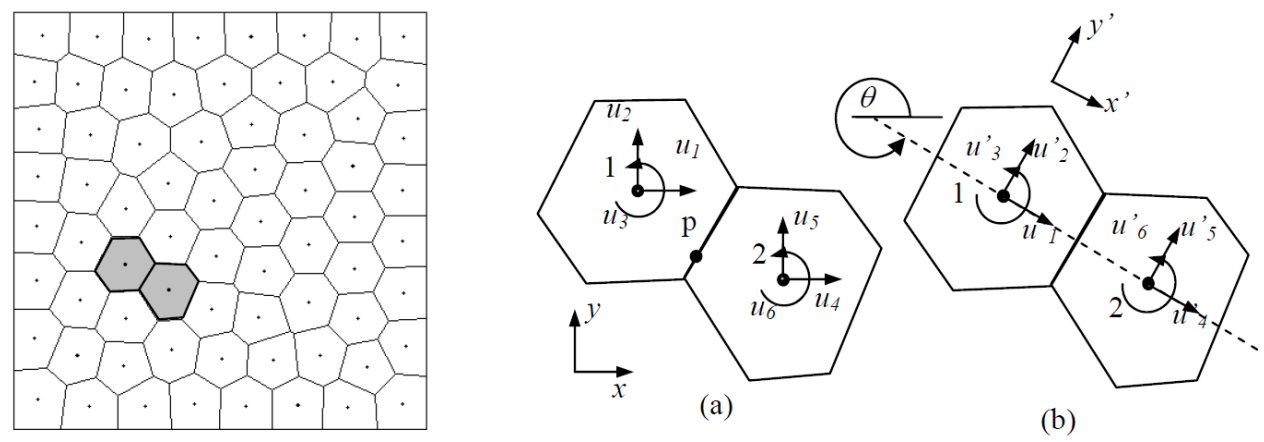

Figure 4.9: Element Assembly in VAEM [59]

rigid particle has two translational and a rotational degree of freedom defined at the particle centroid. For small rotations, the motion of any two points (x,y) of a rigid body can be defined as in Equation 4.16

$$
\begin{aligned}
& u_{1}=u_{c 1}-u_{3}\left(y-y_{c 1}\right) ; u_{2}=u_{c 2}+u_{3}\left(x-x_{c 1}\right) \\
& u_{4}=u_{c 4}-u_{6}\left(y-y_{c 2}\right) ; u_{5}=u_{c 5}+u_{6}\left(x-x_{c 2}\right)
\end{aligned}
$$

where $u_{1}, u_{2}, u_{3}, u_{4}, u_{5}, u_{6}$, are translational displacements and rotation angles of elements 1 and 3 in the global coordinate system. The subscript $c$ specifies the value at the particle centroid. The relative displacement vector of spring 
deformation in the global coordinate system at point $p$ can be defined as Equation 4.17

$$
\left\{\delta_{g}\right\}=\overrightarrow{p^{\prime} p^{\prime \prime}}=\left\{\begin{array}{l}
\delta_{x} \\
\delta_{y}
\end{array}\right\}=\left\{\begin{array}{l}
u_{4}-u_{1} \\
u_{5}-u_{2}
\end{array}\right\}
$$

Substituting Equation 4.16 in 4.17 and rotating the displacement to the local coordinates parallel to the element surface, the relationship between the spring deformation in the local coordinates and particle displacement in the global coordinates is obtained, shown in Equation 4.18

$$
\left\{\delta_{t}\right\}=[R][B]\{u\}
$$

where $\left\{\delta_{t}\right\}^{T}=\left[\delta_{n}, \delta_{t}\right]$ in which $\delta_{n}$ and $\delta_{t}$ are the normal and shear deformation of a spring respectively, and $[R]$ is the rotational matrix

$$
[B]=\left[\begin{array}{cccccc}
-1 & 0 & \left(y-y_{c 1}\right) & 1 & 0 & -\left(y-y_{c 2}\right) \\
0 & -1 & -\left(x-x_{c 1}\right) & 0 & 1 & \left(x-x_{c 2}\right)
\end{array}\right]
$$

and $\{u\}^{T}=\left[u_{1}, u_{2}, u_{3}, u_{4}, u_{5}, u_{6}\right]$.

\subsection{Conclusion}

The complete formulation of the Applied Element Method was presented in this chapter, along with modifications to the method. The AEM showed to have some advantages in modelling structural behaviour. Some of the advantages include the ease of modelling structures with multiple materials, such as reinforced concrete, since the material properties are represented in the springs. The AEM was examined for small displacements, large displacements, and modification to element shape and the stiffness for improvements of the method, which are summarised as follows.

An analysis run by Meguro and Tagel-Din was presented to show the effect of the number of elements and the number of springs in a structure and how they affect computational cost and accuracy. The study highlighted very important 
factors that must be considered. It was mentioned that the number of springs did not affect the element stiffness, when only considering the translational degreesof-freedom. Problems must arise when bending is considered. It was concluded that at least 10 springs where needed per element in order to reduce the rotational stiffness error. So it was suggested to use 10 springs per element and use smaller sized elements as errors arise in the rotational stiffness.

The AEM was also examined for validation of large displacement analysis. Residual force vectors are needed to be considered in this analysis. Some limitations were presented which were, symmetry of structure must avoided in buckling analysis. Small increments of load must be assumed since the small displacement theory is implemented. Moreover, the stiffness of the structure decreases after buckling.

Different modifications in the last decades have been made to the original AEM. One of the modifications presented is the Improved Applied Element Method. The major advantage is its capability of accurately analysing shear stiffness without requiring a large number of springs. The modification is implemented by having different values of $t$ thickness for each spring. The modification allows modelling different cross-sectional elements such as I-beams, box and channel sections.

Another modification to the AEM is the change in the shape of the elements. Rather than using square elements, the element formulation is based on the Voronoi tessellation. The advantage of using Voronoi elements is that meshing of structures is not limited to square shaped elements.

From this chapter it is concluded that there is a need for optimization of the number or springs required to successfully model beam bending accurately without requiring a large number of springs or elements. An optimization solution to this problem will be presented in the following chapter using Gaussian quadrature for spring distribution. 


\section{Chapter 5}

\section{Gaussian based AEM}

\section{Formulation}

In this chapter structural elements in linear elastic cases using the AEM are presented. In the scope of this research, the AEM is programmed using MATLAB.

As mentioned in the previous chapter, the number of springs required in the system is quite large for linear problems in the case that rotational stiffness needs to be considered. A number of at least 10 springs were required with the limitation that the element size is also relatively small. Solving linear elastic problems should be simple enough to use larger number of elements and less number of springs to optimise the computational cost. An optimisation solution is presented in this chapter by modifying the AEM springs distribution using the Gaussian quadrature.

This chapter entails two parts. First the AEM with equally distributed springs is modelled using MATLAB. The conventional AEM is compared with the FEM which is analysed on ANSYS Workbench. The two methods will be compared for validation purposes. The second part of this chapter addresses the problem of using many springs per element. An optimisation modification to the AEM is presented: a new distribution for the springs based on the Gaussian quadrature in section 5.3. 


\subsection{AEM for Linear Elastic Cases}

As previously mentioned, the AEM is coded on MATLAB. The algorithm for a linear elastic beam analysis is split into the pre-processing, processing and post-processing stage. In the preprocessing stage, user defined input is required: determining the geometrical and material properties of the beam, the load applied and its location, as well as the boundary conditions. Given the span and the number of elements required, the meshing of the beam can be done using the meshgrid function on MATLAB, where the element centres are obtained, along with the vertices of the elements. The connectivities between elements are then obtained based on finding elements with shared vertices. The springs locations are then determined based on the number of springs between elements, and if elements are connected in all directions. The processing stage then commences. There are two loops in the code: the first loop goes through every pair of connected elements, while the second one loops through every pair of springs to calculate the stresses and strains in each spring.

Consider the cantilever beam shown in Figure 5.1 as an example. The code will loop through every pair of elements; $1-2,2-3,3-4,1-9,2-10,9-10$, etc. In each element loop, there is a loop to pass through all the springs between each pair of elements. For the simplicity and reduction of redundancy in analysis, every pair of elements is analysed locally and then transformed back to the global matrix. The elements are transformed to the local coordinates which have the orientation shown in Figure 5.2 and then the loop through every spring is done. The stiffness matrix for each spring is obtained and added to the global stiffness matrix. For example, the global element 1-9 is transformed from the local coordinates to global coordinates, as shown in Figure 5.2. The computational algorithm for analysing beam deflection in the elastic stage is shown in Box 5.1. 


\section{Box. 5.1.1: Applied Element method linear algorithm}

1. Input: Geometric and material properties, boundary conditions, load application and method properties (number of elements, springs, etc.)

2. Calculate global springs locations

3. Loop through every pair of connected elements

(a) obtain nodes, dof's and coordinates of springs

(b) transform global coordinates to local

i. Loop through every spring

ii. Calculate stiffness matrix

(c) Assemble in Global stiffness matrix

4. Calculate displacement

$$
u=[K]^{-1}\left(f_{\text {ext }}\right)
$$

5. Calculate stress and strain from obtained deflection

\begin{tabular}{|c|c|c|c|c|c|c|c|}
\hline 1 & 2 & 3 & 4 & 5 & 6 & 7 & 8 \\
\hline 9 & 10 & 11 & 12 & 13 & 14 & 15 & 16 \\
\hline
\end{tabular}

Figure 5.1: Noded cantilever beam diagram
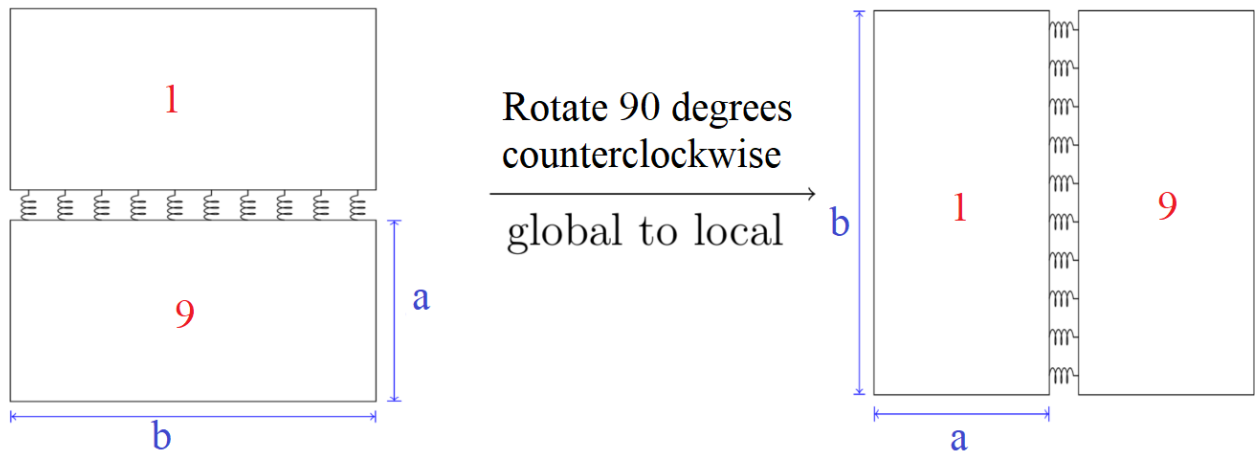

Figure 5.2: Local Orientation

The computational method is efficient and can successfully obtain the displacement between elements. If the load is applied in one time step, and no further time steps are considered or load increments implemented, then the beam is analysed by calculating the stiffness of each spring and implementing the stiffness in the local stiffness matrix, that is eventually assembled in the global stiffness matrix. When the stiffness matrix of the whole system is obtained, the deflection 
can be calculated along with corresponding stresses and strains in the system due to the applied load.

\subsection{Linear Elastic Case Studies: AEM vs. FEM}

This section entails the verification of the AEM code written and compared to FEM and analytical solutions, in order to quantify the accuracy of the method. In this section, simple structural elements will be analysed using AEM and compared to the finite element method using ANSYS. Analysis will be done to also find the optimal number of elements and springs needed in AEM to acquire a good level of convergence. The load in these examples is applied in one time step. No load increments are presented.

\subsubsection{Cantilever Beam}

Assume a cantilever beam, fixed at one end and free at the other, with a point load applied at the free end, as displayed in Figure 8.5. The material properties of the beam are shown in Table 5.1, which are of steel. The load applied is very small to allow only small deformations and elastic material behaviour. The beam will initially be analysed using the same number of elements, in both AEM and in FEM. Then a comparison of the number of elements to springs using AEM will be displayed.

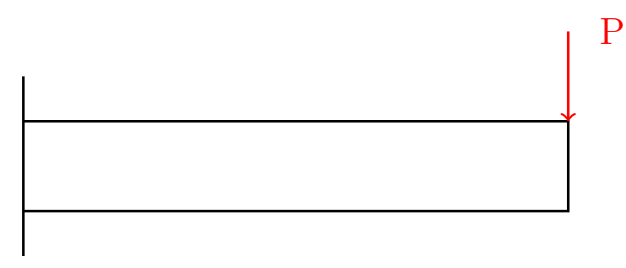

Figure 5.3: Cantilever Free Body Diagram

\begin{tabular}{c|cc} 
Modulus of Elasticity E & 200,000 & {$[\mathrm{MPa}]$} \\
Shear Modulus of Elasticity G & 76,923 & {$[\mathrm{MPa}]$} \\
Yield Stress & 250 & {$[\mathrm{MPa}]$} \\
Beam span & 1 & {$[\mathrm{~m}]$} \\
cross-section width & 0.15 & {$[\mathrm{~m}]$} \\
cross-section thickness & 0.15 & {$[\mathrm{~m}]$} \\
Applied Load & 1000 & {$[\mathrm{~N}]$}
\end{tabular}

Table 5.1: Beam Properties 


\section{Analytical Solution}

For a cantilever beam, with a point load at the free end, the rotation, deflection at any point and the maximum deflection, are shown in Equations 5.2 - 5.4.

$$
\begin{array}{r}
\theta=\frac{P l^{2}}{2 E I} \\
y=\frac{P x^{2}}{6 E I}(3 l-x) \\
\delta_{\max }=\frac{P l^{3}}{3 E I}
\end{array}
$$

where $l$ is the beam length; $P$ is the applied load, $x$ is the point on the beam at which the deflection is to be calculated; $E$ is the Modulus of Elasticity, and $I$ is the moment of inertia. For an applied load of $1 \mathrm{kN}$ applied at the free end, the deflection at the free end is

$$
\delta_{\max }=\frac{1000 \times 1^{3}}{3 * 210 \times 10^{9} * 4.2187 \times 10^{-} 05}=3.76 \times 10^{-5}[\mathrm{~m}]
$$

This deflection will be used for comparison with the solutions obtained from AEM and FEM.

\section{Computational Solutions}

The FEM solution is obtained using ANSYS. The beam deflection and normal stresses are compared. Assuming a beam with an initial number of 10 elements in the $\mathrm{x}$-direction and 1 element in the $\mathrm{y}$-direction, the beam deflection from ANSYS is shown in Figure 5.4, and from the MATLAB code using AEM in Figure 5.5. As seen from the figures, the distribution of the deflection is the same. The reason the FEM results appear with a higher deflection is due to the factor that the deflection is multiplied by to extenuate the deflection for better visibility.

The analytical solution for deflection at the free end of the the cantilever compared with FEM and AEM is shown in Table 5.2. The results of both the FEM and AEM showed good accordance in comparison to the analytical solution.

Figure 5.6 is the analysis of the cantilever beam from the example in Figure 


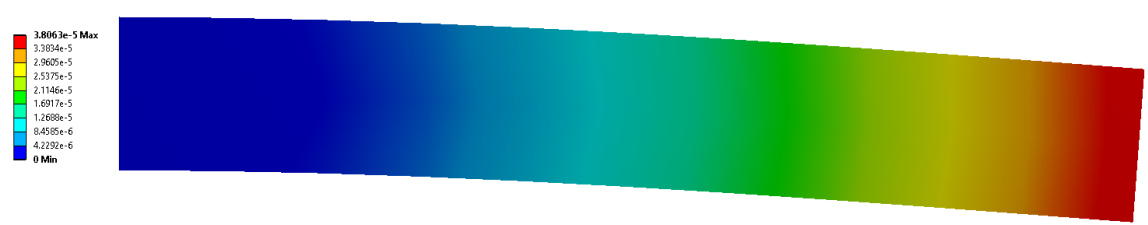

Figure 5.4: ANSYS Cantilever Deflection

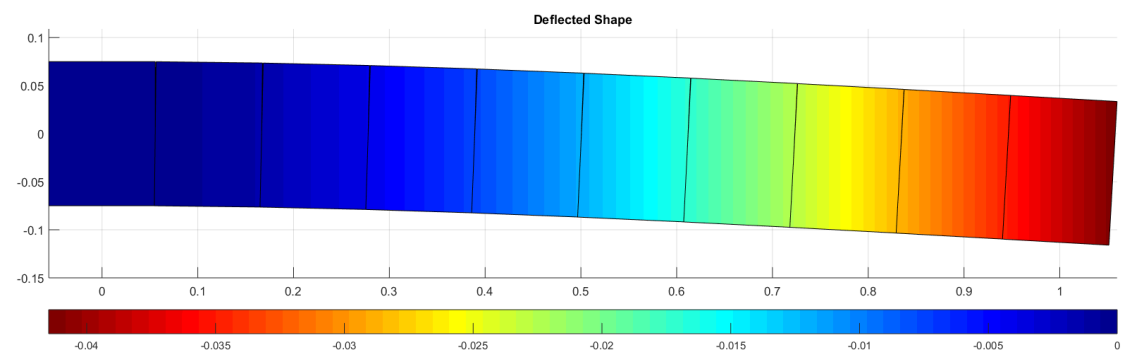

Figure 5.5: MATLAB Cantilever Deflection

Table 5.2: Small Displacement comparison

\begin{tabular}{c||ccc} 
& FEM $[\mathrm{m}]$ & AEM $[\mathrm{m}]$ & Analytical $[\mathrm{m}]$ \\
\hline Deflection & $3.82 \times 10^{-5}$ & $3.88 \times 10^{-5}$ & $3.78 \times 10^{-5}$ \\
\hline
\end{tabular}

5.5, with the load applied in the positive direction. The purpose is to validate that the deflection is the same in the opposite direction. This shows that the elements are reacting to the load in the correct way.

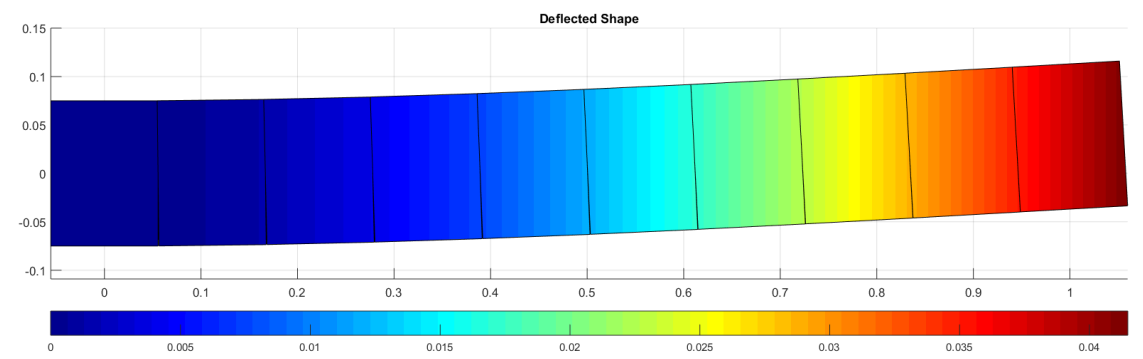

Figure 5.6: MATLAB Cantilever Deflection 10 elements in x-direction opposite load

\section{Normal Stress}

The stresses of the springs are calculated after the deflection is obtained. Figure 5.7 is the stress at each spring, for every cross section between elements. The 
stresses show that the behaviour of all elements are in the linear elastic region. It can be seen that the stress distribution at each cross-section is exactly symmetric, and at the neutral axis, the stress is zero.

Figure 5.8 is the normal stress distribution across the beam from ANSYS, and Figure 5.9 displays the results from the AEM MATLAB code. A visual comparison of the figures shows that the results are almost exact. The absolute value of the normal stress is shown in the figures (this is why the red appears in both the top and bottom fibres at the sections closest to the fixed support). The maximum stress is $1.6 \times 10^{6}$ for both the FEM and the AEM.

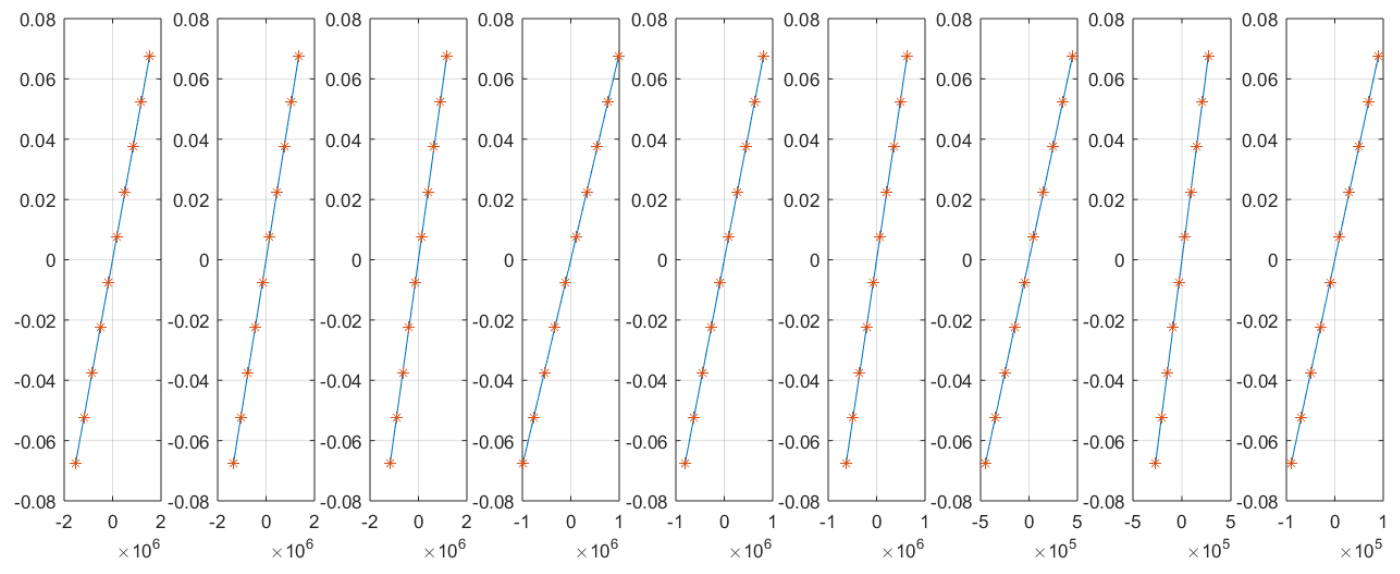

Figure 5.7: MATLAB Cantilever Normal Stress

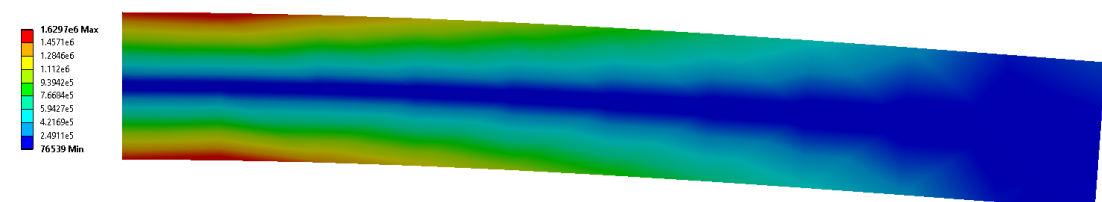

Figure 5.8: ANSYS stress distribution

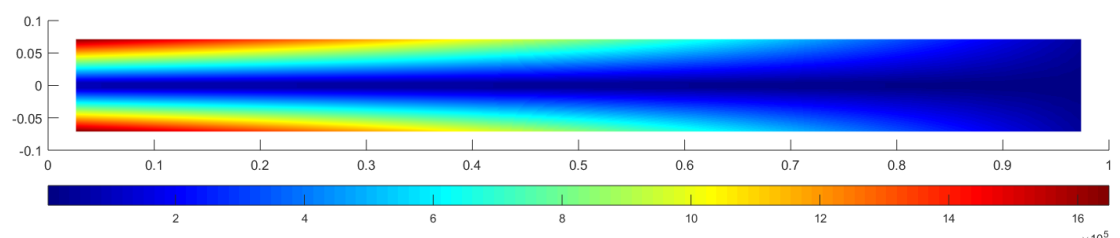

Figure 5.9: AEM stress distribution

\section{Shear Stress}

The maximum shear stress from the ANSYS was $4.8 \times 10^{4}$, while it was $4.445 \times 10^{4}$ from the AEM. The shear stress is constant across the element. Figure 5.10 is 
the shear stress using FEM, and Figure 5.11 is the shear stress from the AEM analysis. The results are quite similar as the stress is almost constant throughout the whole beam, except for the ends where the load is applied.

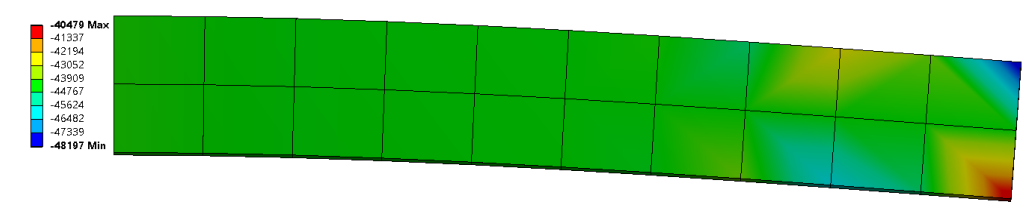

Figure 5.10: ANSYS shear stress distribution

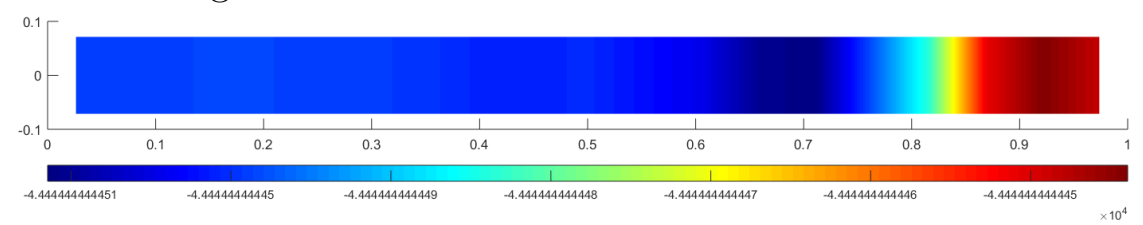

Figure 5.11: AEM shear stress distribution

\section{Validation of Multilayer Mesh}

A multilayer mesh is used to analyse the same cantilever beam as the previous sections. Recall that an element connected in all four edges will need have four contributions to the stiffness matrix. The purpose of this example is to ensure that the elements are transformed from the local to global coordinates correctly, and that adding the extra elements in the y-direction does not cause any errors. Figure 5.12 displays the deflection of the beam with the load applied in the negative y-direction. The deflection is compared to the FEM analysis and similar results were obtained. Figure 5.13 shows the deflection of the beam with the load applied in the positive y-direction. The deflection is exactly the same for the positive and negative load. This is a good indication that the system can successfully model elements that are presented in any direction.

\subsubsection{Fixed End Beam}

Similar to the cantilever beam analysis, a fixed end beam is analysed. The fixed end beam is fixed at both ends. A point load is applied at the midspan. The 


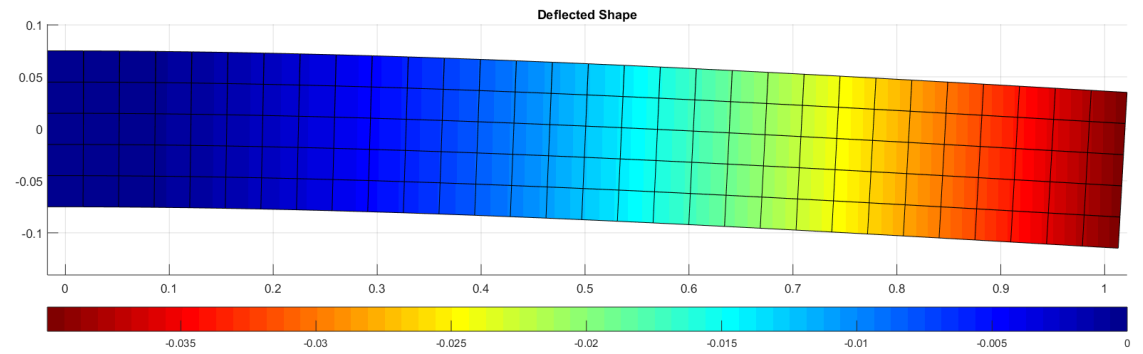

Figure 5.12: AEM Cantilever Deflection 30 elements in x, 5 elements in y

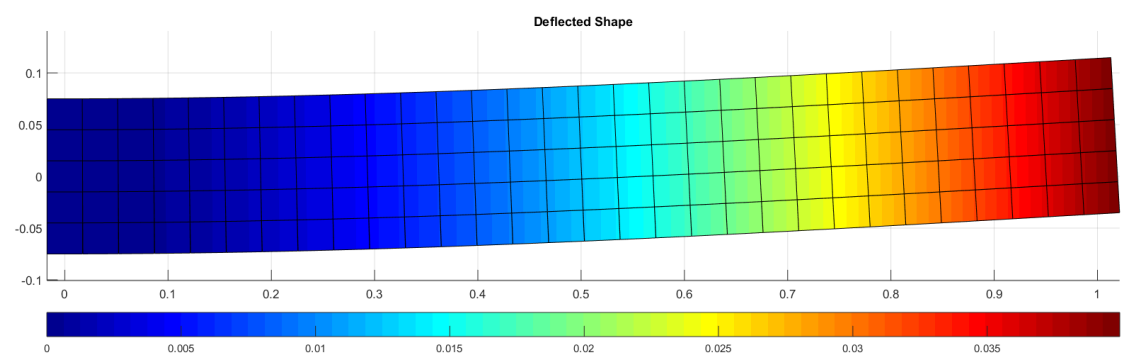

Figure 5.13: AEM Cantilever Deflection Load Positive Direction

beam properties are the same as the properties shown in Figure 5.1.

\section{Analytical Solution}

The deflection at the location of the applied load is shown in Equation 5.6.

$$
\delta_{\max }=\frac{P l^{3}}{192 E I}
$$

For an applied load of $1 k N$ the deflection at the location of the load is

$$
\delta_{\max }=\frac{1000 * 1^{3}}{192 * 210 \times 10^{9} * 4.2187 \times 10^{-} 05}=5.88 \times 10^{-07}[\mathrm{~m}]
$$

This deflection will be used for comparison with the solutions obtained from AEM and FEM.

\section{Computational Solution}

The deflection of the fixed end beam from MATLAB is shown in Figure 5.15, where the results are scaled up to show the deflection of the beam since the displacement is very small. The beam deflection from the FEM analysis is shown 
in Figure 5.14. The results in Table 5.3 show that both the FEM and AEM have similar results. However there is a difference with the analytical result. The number of elements in both the FEM and AEM analysis were exactly the same: 10 elements along the $\mathrm{x}$-direction and 2 elements along the $\mathrm{y}$-direction.

Table 5.3: FEB deflection Comparison

\begin{tabular}{l||ccc} 
& FEM & AEM & Analytical \\
\hline Deflection & $7.04 \times 10^{-} 7$ & $7.8 \times 10^{-} 7$ & $5.88 \times 10^{-07}$ \\
\hline
\end{tabular}

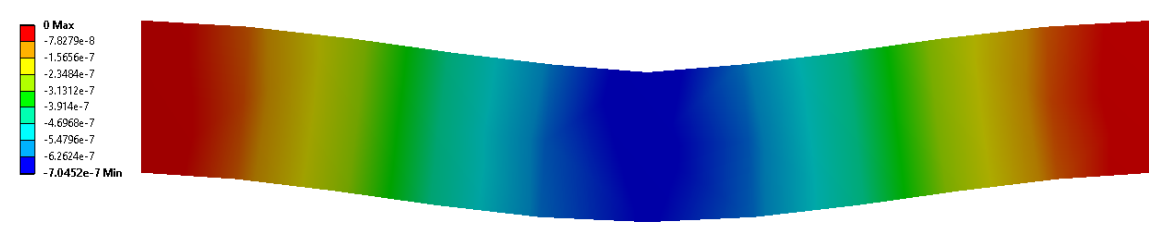

Figure 5.14: ANSYS Fixed End Beam Deflection
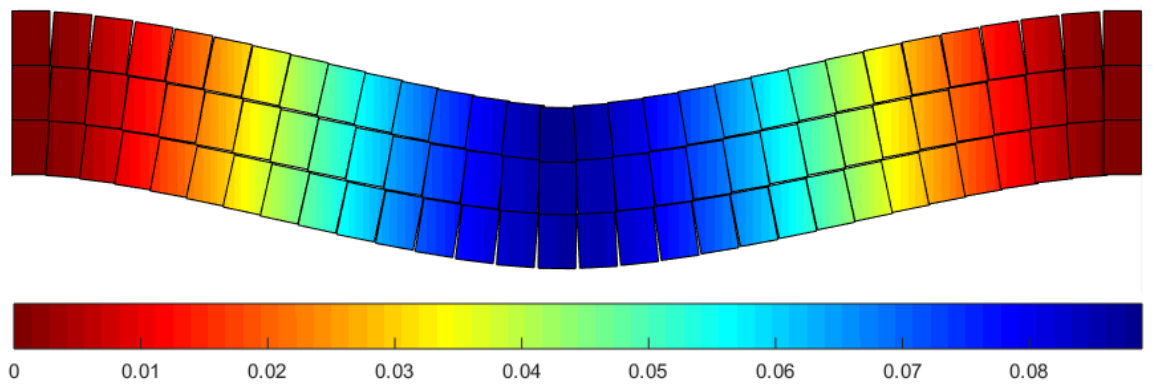

Figure 5.15: AEM Fixed End Beam Deflection

Figure 5.17 is the normal stress along the beam cross-section from the AEM in MATLAB, and Figure 5.16 displays the stress from ANSYS. The maximum stress from the AEM was $1.95 \times 10^{5}[\mathrm{~Pa}]$ and from ANSYS was $1.81 \times 10^{5}[\mathrm{~Pa}]$.

Figures $5.18 \mathrm{a}$ and $5.18 \mathrm{~b}$ display the deflection of the beam with more elements in the y-direction. The results were exactly the same in both cases of applied loads in opposite directions.

\subsubsection{Simply Supported Beam}

A simply supported beam is also analysed. The boundary conditions consist of a constrained $\mathrm{x}$ degree-of-freedom on one side, and the $\mathrm{x}$ and $\mathrm{y}$ degrees-of-freedom on the other side. 
Figure 5.16: Normal stress for FEB from ANSYS

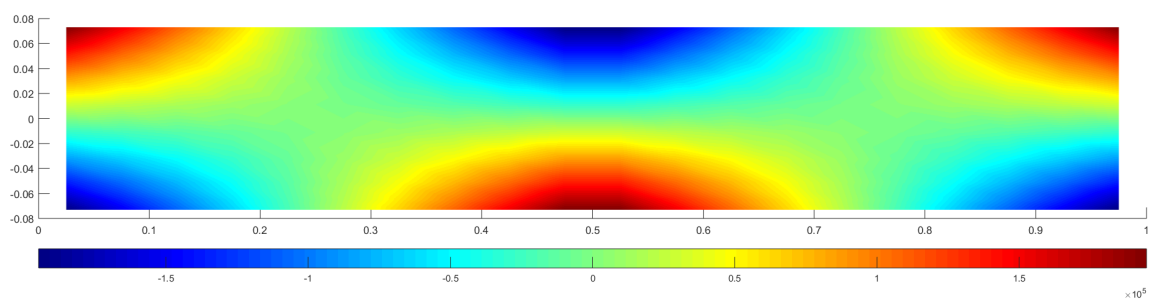

Figure 5.17: Normal stress for FEB from AEM

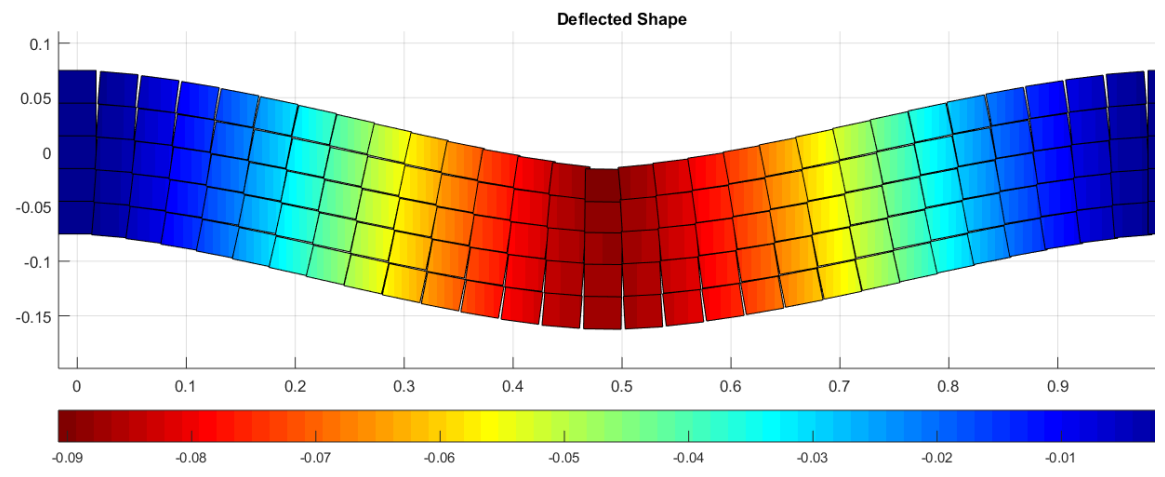

(a) Negative load

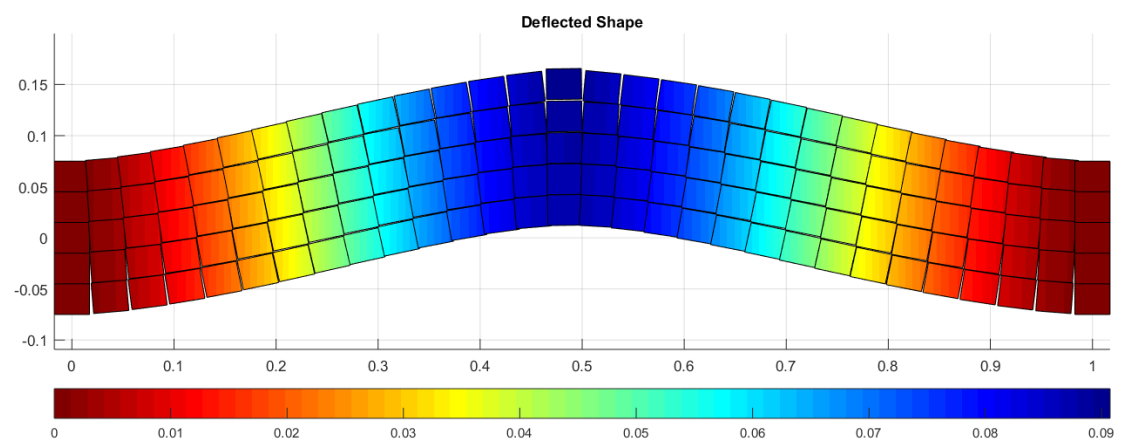

(b) Positive load

Figure 5.18: AEM Different loading direction for FEB 


\section{Analytical Solution}

The analytical solution for the deflection of a simply supported beam with a point load at the midspan is

$$
\delta_{\max }=\frac{P L^{3}}{48 E I}
$$

In this case, for a point load of $1 \mathrm{kN}$, the deflection at the midspan is

$$
\delta_{\max }=\frac{1000 * 1^{3}}{48 * 210 \times 10^{9} * 4.2187 \times 10^{-5}}=2.35 \times 10^{-6}[\mathrm{~m}]
$$

\section{Computational Solution}

Figure 5.19 is the y-deflection of the beam scaled up by a factor of $10^{3}$, and Figure 5.20 is the absolute value of the normal stress of the beam.

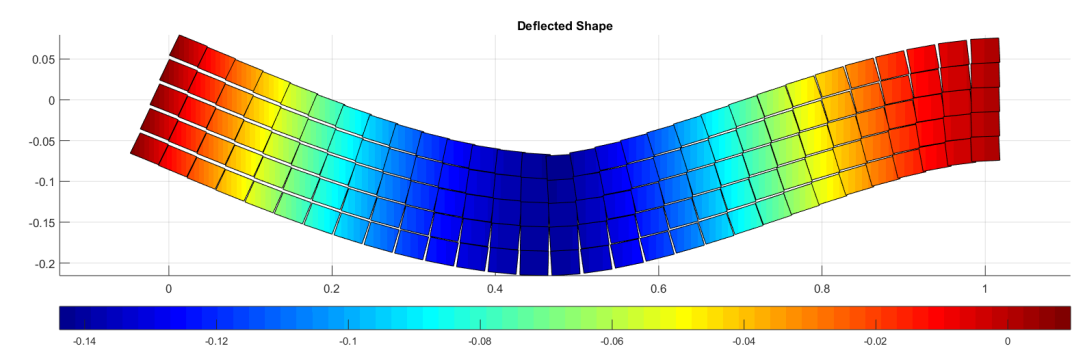

Figure 5.19: AEM Deflection Simply Supported Beam

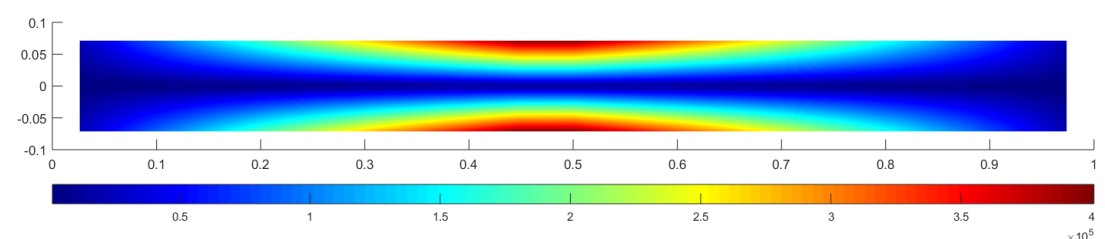

Figure 5.20: AEM Normal stress Simply Supported Beam

\subsection{Gaussian Springs based AEM}

For a beam, the exact second moment of inertia is needed for the stress calculation, and using the Gaussian quadrature rule with $n=2$ points will integrate a polynomial of order $2 n-1=3$ exactly. Since the moment of inertia is of order 
2, 2 points were sufficient. However, 50 equally spaced springs where required to obtain the same results.

Gaussian quadrature is used for finite element applications because it has fewer function evaluations for given orders. The weights and evaluation points are determined so that the integration rule is exact to as high an order as possible Gaussian quadrature of order $\mathrm{N}$ for the standard interval $[-1,1]$, is given by

$$
\int_{-1}^{1} g(\xi) d(\xi)=\sum_{i=1}^{N} w_{i} g\left(\xi_{i}\right)
$$

where $\xi_{i}$ and $w_{i}$ are the Gaussian quadrature points and weights. A Gaussian quadrature using $\mathrm{N}$ points can provide the exact integral if $g(\xi)$ is a polynomial of the degree $2 N-1$ or less. The Gaussian quadrature for the general integral $\mathrm{I}=[\mathrm{a}, \mathrm{b}]$ is given by

$$
\int_{a}^{b} f(x) d x=\frac{b-a}{2} \int_{-1}^{1} f\left(\frac{b-a}{2} x+\frac{b+a}{2}\right) d x=\frac{b-a}{2} \sum_{i=1}^{n} W_{i} f\left(x_{i}\right)
$$

\subsubsection{Gaussian Springs Implementation in AEM}

The Gaussian Quadrature is implemented to a pair of 2-D AEM elements to determine the Gaussian weights and coordinates using the number of springs. The location of the springs is determined by considering $w$ as the width of the tributary area of each spring (' $d$ ' in AEM Equation 4.5), and $x$ as the spring location from Equation 5.11.

Figure 5.21a and Figure 5.21b show the comparison of the tributary area when the distribution of the springs is equal (left), and springs are distributed using the Gaussian quadrature (on the right).

Table 5.4 shows the Gaussian and Equal spring location, $x_{i}$, and weighting factor, $w_{i}$, of each spring $i$ for 3 springs, and Table 5.6 is the distribution for 5 springs. $x_{i e}$ is the spring location for an equal spring, and $x_{i g}$ is for a Gaussian spring. Similarly for $w_{i e}$ and $w_{i g}$.

Assuming the width of the section is 5 , then $x_{i}$ and $w_{i}$ are multiplied by $5 / 2=2.5$. The multiplication is presented in Tables 5.5 and 5.7 for 3 and 5 springs, respectively. Looking at row $2.5 w_{i e}$ and $2.5 w_{i g}$, a clear distinction of the 


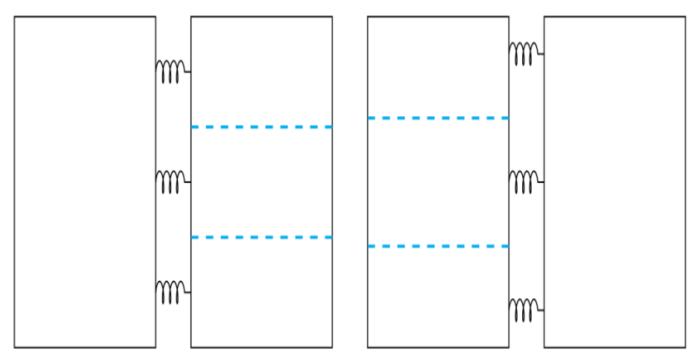

(a) 3 springs - Equal (left), Gaussian (right)

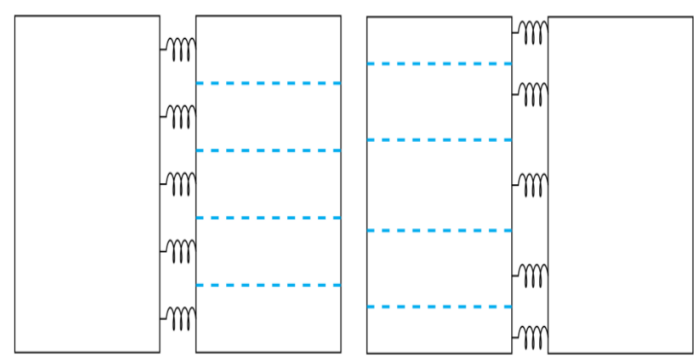

(b) 5 springs - Equal (left), Gaussian (right)

Figure 5.21: Comparison of Gaussian and Equal Springs Tributary Area for 3 and 5 springs

weight of each spring is shown. For instance for the 3 springs, the middle spring (point 2), has a weighted factor of 1.67 in the equal distribution rather than 2.2 in the Gaussian distribution.

Table 5.4: 3 springs coordinates and weighting factor

\begin{tabular}{c|cc|cc} 
Point & $x_{i e}$ & $w_{i e}$ & $x_{i g}$ & $w_{i g}$ \\
\hline 1 & -0.667 & 0.67 & -0.775 & 0.556 \\
2 & 0 & 0.67 & 0 & 0.889 \\
3 & 0.667 & 0.67 & 0.775 & 0.556 \\
\hline
\end{tabular}

Table 5.6: 5 springs coordinates and weighting factor

\begin{tabular}{c|cc|cc} 
Point & $2.5 x_{i} e$ & $2.5 w_{i} e$ & $2.5 x_{i} g$ & $2.5 w_{i} g$ \\
\hline 1 & -2 & 1 & -1.359 & 0.355 \\
2 & -1 & 1 & -0.808 & 0.718 \\
3 & 0 & 1 & 0 & 0.853 \\
4 & 1 & 1 & 0.808 & 0.718 \\
5 & 2 & 1 & 1.359 & 0.355 \\
\hline
\end{tabular}

Table 5.5: 3 springs $w_{i}$ and $x_{i}$ for crosssection $5 \mathrm{~m}$

\begin{tabular}{c|cc|cc} 
Point & $2.5 x_{i e}$ & $2.5 w_{i e}$ & $2.5 x_{i g}$ & $2.5 w_{i g}$ \\
\hline 1 & -1.668 & 1.67 & -1.937 & 1.389 \\
2 & 0 & 1.67 & 0 & 2.222 \\
3 & 1.668 & 1.67 & 1.937 & 1.389 \\
\hline
\end{tabular}

Table 5.7: 5 springs coordinates and weighting factor

\begin{tabular}{c|cc|cc} 
Point & $x_{i} e$ & $w_{i} e$ & $x_{i} g$ & $w_{i} g$ \\
\hline 1 & -0.8 & 0.4 & -0.906 & 0.237 \\
2 & -0.4 & 0.4 & -0.538 & 0.479 \\
3 & 0 & 0.4 & 0 & 0.569 \\
4 & 0.4 & 0.4 & 0.538 & 0.479 \\
5 & 0.8 & 0.4 & 0.906 & 0.237 \\
\hline
\end{tabular}

\subsubsection{Comparison of equal springs with Gaussian springs}

A comparison of equal and Gaussian springs will be shown to prevail the impact the distribution of the springs can have on the accuracy of the solution. As before, a cantilever beam and a fixed end beam analysis will be done, with a small linear-elastic load. 


\section{Cantilever Beam}

Consider a cantilever beam with the similar properties shown from Table 5.1, and a point load applied at the free end. The only difference in the MATLAB code occurs in the spring distribution definition. For a load of $1 \mathrm{kN}$, a comparison of the beam deflection is shown in Table 5.8, for an equal number of 10 elements along the $\mathrm{x}$-direction, and varying springs. The results show that using the Gaussian springs distribution with just 2 springs gives almost the same deflection that was calculated with 50 equally distributed springs. The error percentage presented is the difference in the deflection at the free between the result obtained from the equal springs distribution and the Gaussian springs distribution. Therefore, a beam with 10 elements, requires a total of $10 \times 2=20$ Gaussian springs, or $10 \times 50=500$ equal springs.

Table 5.8: Deflection at free end comparison

\begin{tabular}{c||c|c|c|c|c|c|} 
No. springs & 2 & 5 & 10 & 15 & 30 & 50 \\
\hline Equal & 0.4215 & 0.3295 & 0.3196 & 0.3179 & 0.3168 & 0.3165 \\
Gaussian & 0.3164 & 0.3164 & 0.3164 & 0.3164 & 0.3164 & 0.3164 \\
\hline Difference & $24.9 \%$ & $4.0 \%$ & $1.0 \%$ & $0.5 \%$ & $0.1 \%$ & $0.0 \%$
\end{tabular}

Table 5.9 displays the y-deflection at each node of the beam using 2 equallydistributed springs, and 2 Gaussian springs. The y-deflection in the first element is not shown since it is fixed to zero as a boundary condition. The results show that the percentage difference in the deflection is quite different for every element, almost 25\%. Table 5.10 displays the y-deflection at each node, with 50 springs per element. It can be seen that the beam deflection is almost exactly the same at every node.

It is also important to compare the data of the deflection of the elements using 2 Gaussian springs and 50 Gaussian springs, displayed in Table 5.11. It is clear that the results are exactly the same. This verifies that using Guassian springs is much more efficient than using equally distributed springs, since the deflection of all the elements along the beam is the same.

\begin{tabular}{|l|l|l|l|l|l|l|l|l|l|}
\hline 1 & 2 & 3 & 4 & 5 & 6 & 7 & 8 & 9 & 10 \\
\hline
\end{tabular}


Figure 5.22: Beam with 10 elements

Table 5.9: y-deflection of beam at every node - 2 springs

\begin{tabular}{c||c|c|c|c|c|c} 
Element & 2 & 4 & 5 & 6 & 8 & 10 \\
\hline Equal & -0.0075 & -0.0624 & -0.1063 & -0.1589 & -0.2831 & -0.4213 \\
Gaussian & -0.0057 & -0.0469 & -0.0799 & -0.1193 & -0.2126 & -0.3162 \\
\hline Difference & $24.0 \%$ & $24.8 \%$ & $24.8 \%$ & $24.9 \%$ & $24.9 \%$ & $24.9 \%$
\end{tabular}

Table 5.10: y-deflection of beam at every node - 50 springs

\begin{tabular}{c||c|c|c|c|c|c} 
Element & 2 & 4 & 5 & 6 & 8 & 10 \\
\hline Equal & -0.0057 & -0.0469 & -0.0799 & -0.1194 & -0.2127 & -0.3164 \\
Gaussian & -0.0057 & -0.0469 & -0.0799 & -0.1193 & -0.2126 & -0.3162 \\
\hline Difference & 0 & 0 & 0 & $-0.08 \%$ & $-0.05 \%$ & $-0.06 \%$
\end{tabular}

Table 5.11: y-deflection of beam at every node - 2 and 50 Gaussian springs

\begin{tabular}{c||c|c|c|c|c|c} 
Element & 2 & 4 & 5 & 6 & 8 & 10 \\
\hline 2 springs & -0.0057 & -0.0469 & -0.0799 & -0.1193 & -0.2126 & -0.3162 \\
50 springs & -0.0057 & -0.0469 & -0.0799 & -0.1193 & -0.2126 & -0.3162 \\
\hline Difference & 0 & 0 & 0 & 0 & 0 & 0
\end{tabular}

\section{Fixed End Beam}

An analysis similar to that of the cantilever beam will be done for a fixed end beam. Tables 5.12 and 5.13, display the deflection in the y-direction of every element in the beam for elements with 2 springs and 50 springs respectively. The results show that the use of Gaussian springs is much more efficient than equally distributed springs. Elements 1 and 10 have a boundary condition of fixed displacement of 0 , so they are not shown in the tables.

\begin{tabular}{|l|l|l|l|l|l|l|l|l|l|}
\hline 1 & 2 & 3 & 4 & 5 & 6 & 7 & 8 & 9 & 10 \\
\hline
\end{tabular}

Figure 5.23: Fixed End Beam with 10 elements

Table 5.12: y-deflection of FEB at every node - 2 springs

\begin{tabular}{c||c|c|c|c|c|c} 
Element & 2 & 4 & 5 & 6 & 8 & 9 \\
\hline Equal & -0.0449 & -0.279 & -0.375 & -0.395 & -0.182 & -0.055 \\
Gaussian & -0.0341 & -0.211 & -0.283 & -0.299 & -0.139 & -0.042 \\
\hline Difference & $31.67 \%$ & $32.54 \%$ & $32.54 \%$ & $32.37 \%$ & $32.29 \%$ & $31.73 \%$
\end{tabular}


Table 5.13: y-deflection of FEB at every node - 50 springs

\begin{tabular}{c||c|c|c|c|c|c} 
Element & 2 & 4 & 5 & 6 & 8 & 9 \\
\hline Equal & -0.034 & -0.211 & -0.283 & -0.299 & -0.137 & -0.0417 \\
Gaussian & -0.034 & -0.211 & -0.283 & $-0.299-0.239$ & -0.137 & -0.0416 \\
\hline Difference & 0 & 0 & $0.035 \%$ & $0.033 \%$ & $0.073 \%$ & $0.240 \%$
\end{tabular}

Therefore, it can be concluded that using a Gaussian distribution for the springs, rather than an equal spring distribution, can have a significant effect on the number of springs needed to acquire a rapid convergence in terms of solution accuracy, with a significantly low number of springs.

\subsection{Conclusion}

This chapter entailed an analysis difference between the FEM and the AEM, where the AEM was modelled using MATLAB. Analysis of different structural beams (a cantilever beam, a fixed ends beam and a simply supported beam), were performed to prove the validity and accuracy of the method. The maximum deflection of each beam was compared to its respective analytical solution as well as the solution from the finite element analysis, using ANSYS. The shear and normal stresses were also compared. The results showed that the applied element method is a good tool for modelling structural members in the elastic range, and the developed code is valid for future modifications in the method.

A modification to the method was presented in this chapter, since the conventional AEM required a large number of springs and elements in linear elastic solution states. The distribution of the springs by using the Gaussian quadrature, referring to the springs as Gaussian springs, is developed. Instead of using equally distributed springs along element edges, the springs are distributed based on the Gaussian quadrature. The Gaussian springs showed an advantage in the number of springs required to accurately model an elastic behaviour of a structural element.

A cantilever beam with 10 elements and a load of $1 \mathrm{KN}$ applied at the free end was analysed using ANSYS for FEM results, the conventional AEM (with equally spaced springs), and the Gaussian AEM. The findings of the solutions were remarkable. It was found that a total of 50 equally spaced springs were needed to 
obtain the same result as obtained from 2 Gaussian springs when compared to the FEM analysis. A comparison analysis was performed for the cantilever beam deflection of each element with 2 Gaussian springs and 50 Gaussian springs, and it the results showed that the deflection at each element was exactly the same regardless of the number of springs. The comparison analysis was also performed for a fixed ends beam, and the results were just as remarkable. The comparison was performed for analysis between FEM, conventional AEM and Gaussian AEM. When 50 equal springs were used, there was a small discrepancy of $0.03 \%$.

In other words, for the same beam and same number of elements, the conventional AEM required a total of 500 springs, while only 20 springs were required using the Gaussian distribution. This substantially decreases the CPU time required to model the structural element, which in larger scale models will have a much larger effect.

Since the Gaussian springs showed to be much more efficient for modelling structural elements in the elastic region, the model will be extended for analysis of materials subject to elasto-plasticity, in the next chapter. 


\section{Chapter 6}

\section{Gaussian AEM in Elasto-plasticity}

The goal of this research is to model the collapse behaviour of structures. Therefore materials are expected to undergo elasticity, elasto-plasticity, full plasticity, fracture and collapse. To model the complete behaviour from an initial state of no loading until total collapse, all the stages must be considered.

This chapter is an introduction to constitutive models and their applications for material behaviour in elasto-plasticity. A constitutive model is implemented to the MATLAB code, and analysis results are shown for different structural members where comparisons of deflections and stresses are performed. The Gaussian AEM, which was developed in the previous chapter, is implemented for hardening elasto-plasticity and a modification is presented to implement adaptive springs that are based on the yield criteria. The efficiency of the modification is also presented.

\subsection{Overview}

The theory of plasticity is based on solids that sustain permanent deformations when completely unloaded, after being subjected to a loading. This theory is regarded as one of the most successful phenomenological constitutive models of solid materials [68].

Consider a uniaxial tension experiment with a metallic bar in order to il- 
lustrate features of the phenomenological behaviour of materials. The uniaxial tension tests produce stress-strain curves, as shown in Figure 6.1. The axial stress, $\sigma$, is plotted against the axial strain, $\varepsilon$, where the bar is subjected to an increase in axial stress from zero to a prescribed values, $\sigma_{0}$. The bar is then unloaded, and subsequently loaded to a higher stress level, $\sigma_{1}$, and produces the corresponding stress-strain curve. In the initial line segment $O_{0} Y_{0}$, the bar is regarded as linear elastic. Loading and unloading before reaching $Y_{0}$, the bar will remain linearly elastic. After $Y_{0}$ the slope changes and the bar is in plastic range where it will undergo permanent plastic deformation. At point $Z_{0}$ (for instance), if the bar is unloaded it will follow the same linear elastic behaviour as from $O_{0} Y_{0}$. The new permanent shape is observed from the unstressed state $O_{1}$, instead of $O_{0}$.

The vital phenomenological properties from the uniaxial test are as follows [68]: (1) an elastic domain exists, (2) plastic yielding takes place if the material is loaded further than the yield stress, and (3) the evolution of the plastic strain is also observed, known as hardening (in Figure $6.1 Y_{0}$ and $Y_{1}$ are observed to have different yielding stresses). These material behaviours are observed in metals, concrete, soils, rocks and others [68]. This nonlinear material behaviour will be tested in the developed MATLAB code in the following sections.

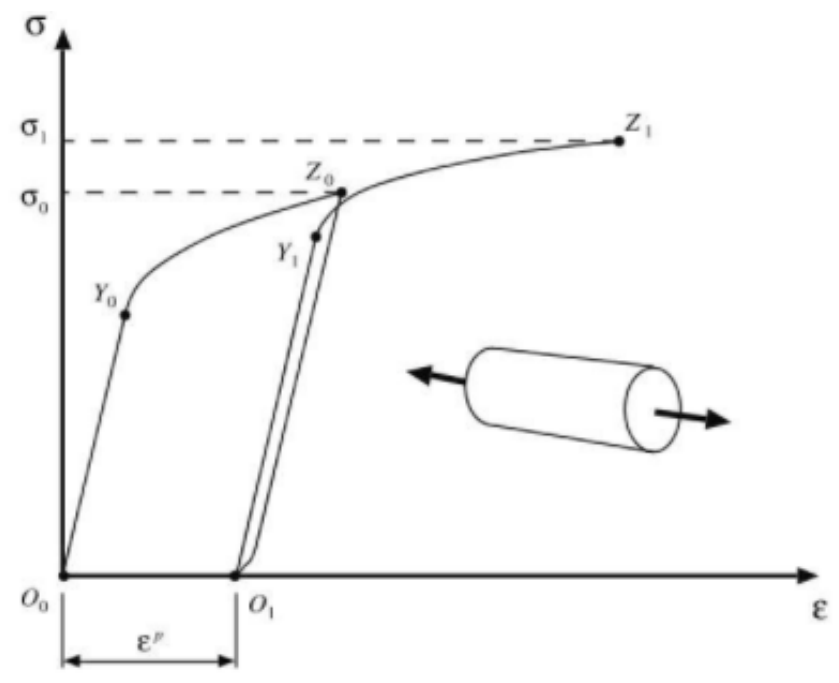

Figure 6.1: Uniaxial Tension Test [68]

In order to represent this material behaviour, mathematical theory of plasticity must be used to demonstrate continuum constitutive models that are capable 
of describing the phenomenological behaviour of materials, quantitatively and qualitatively, with sufficient accuracy. Characterising material behaviour from the initial stages of loading until final stages of failure, or modelling material behaviour with instabilities require complex models of the material behaviour which are obtained through constitutive modelling.

Different engineering materials require different constitutive models due to the difference in the physical mechanisms of the material degradation at the macroscopic level. However, materials show similar mechanical behaviour, such as: elasticity, yielding, plastic strain, strain induced anisotropy, damage by monotonic loading and crack growth. Therefore, models may be developed to display the common behaviour of materials, using continuum mechanics without requiring the complex physical micro structures of the materials, and these models are the constitutive models. In other words, constitutive models are the mathematical simplification of the complex physical behaviour. The mathematical structure of a model depends on the material and the purpose (loading conditions). Different purposes may include [69]:

1. Structural analysis under working load: Linear elasticity

2. Analysis of damped vibrations: Viscoelasticity

3. Calculation of limit load: Rigid perfect plasticity

4. Accurate calculation of permanent deformation after monotonic and cyclic loading: Hardening elasto-plasticity

5. Analysis of stationary creep and relaxation: Perfect (non-hardening) elastoviscoplasticity

6. Prediction of lifetime in high-cycle-fatigue: Damage coupled to elastic deformations

7. Prediction of lifetime in low-cycle-fatigue: Damage coupled to plastic deformations

8. Prediction of lifetime in creep and creep-fatigue: Damage coupled to viscoplastic deformations

9. Prediction of stability of a pre-existing crack: Linear elasticity (from which singular stress fields are derived for sharp cracks)

10. Prediction of strain localization in shear bands and incipient material fail- 
ure: Softening plasticity or damage coupled to plastic deformation

In the scope of this thesis, the linear elasticity, perfect plasticity, hardening elastoplasticity and softening plasticity are demonstrated.

\subsection{Definitions}

In this section, a brief description of how a constitutive model typically works, and the conditions that must be satisfied are explained. The following section entails detailed constitutive equations and derivations. The terminology used in modelling plasticity is presented here and is based on terminology from [70].

Elastic strain, $\varepsilon^{e}$, is any strain that occurs before exceeding the yield stress. The plastic strain, $\varepsilon^{p}$, is the permanent strain that remains after unloading. The total strain is the sum of the elastic strain and plastic strain. When plastic strain is taking place, then it follows that plastic flow is taking place. Internal hardening variables are used when hardening takes place, because when hardening occurs the yield stress changes. The internal variables keep track of the plastic strain. The isotropic internal hardening variable, $\alpha$, is the equivalent plastic strain [71].

A yield condition is used to determine when yielding occurs, denoted by $f$. The yield condition usually includes the current stress minus the initial yield stress added to a function of $\alpha$, which describes the type of hardening. When plastic strain occurs, $\alpha$ is updated. The yield condition $f$ is then calculated, and results in either a positive or negative value.

If a positive result is obtained from the yield condition, then the stress is currently greater then the yield stress, which means that the elastic and plastic strains are increasing. However, $f>0$ is not permitted, and the amount of plastic flow and hardening such that the condition $f=0$ is satisfied must be calculated.

For this, a consistency parameter $\lambda$ is introduced to determine the level of plastic flow and hardening required to achieve the condition $f=0$. The process of elastic or elasto-plastic loading and unloading requires a mathematical description, which led to the use of the Kuhn-Tucker conditions that are used to develop the mathematical algorithms to model the plastic flow [71].

The Khun-Tucker conditions are: $\lambda \geq 0, f(\sigma) \leq 0$ and $\lambda f(\sigma)=0$. Plastic- 
ity algorithms are often used to model nonlinear material behaviour which are incremental in nature. As the load increment increases, the stresses, strains and displacements also increase. The initial displacement is assumed to be in the elastic region, and the resulting new stress is defined as a trial stress. The trial stress is placed in the yield function $f$, and the algorithm is applied. The algorithm "corrects" the trial stress to a new stress if it is not elastic. Assuming the current values of the internal variable $\alpha$ is $\alpha_{n}$, then during the load increment $\alpha$ may increase by $\Delta \alpha$ and, $\alpha_{n+1}=\alpha_{n}+\Delta \alpha$. For this reason, for a rate independent material, the load increment is like a time increment.

Another important issue to consider is that the tangent modulus is equivalent to the elasto-plastic modulus, which is not true in higher dimensions. This causes difficulty in solving the consistency parameter and this issue can be overlooked by using the Newton-Raphson method. To obtain a second order convergence, the derivative of the stress to the total strain must be taken. The algorithmic tangent modulus is defined as $C_{n+1}^{k}=\frac{\partial \sigma_{n+1}^{k}}{\partial \varepsilon_{n+1}^{k}}$. The way that plastic strain evolves in plasticity algorithms is referred to as the flow rule, which takes the form of $\dot{\varepsilon}^{p}=\lambda \frac{d f}{d \sigma}=\lambda \operatorname{sign}(\sigma)$. The isotropic hardening law takes the form of $\dot{\alpha}=\Delta \alpha=\lambda$ $[71]$.

The definitions have now been explained as well as the logic behind the constitutive equations in plasticity. The following section is a derivation of the equations used and implementation of the computational algorithm.

\subsection{Elasto-plastic Constitutive Equations Deriva- tion}

Deformations of elasto-plastic solids at small strains are characterized by the additive split of the total strain

$$
\varepsilon=\varepsilon^{e}+\varepsilon^{p}
$$


where $\varepsilon$ is the total strain, $\varepsilon_{e}$ is the elastic reversible strain, and $\varepsilon_{p}$ is the plastic permanent strain. Hence, the elastic strain can be defined as

$$
\varepsilon^{e}=\varepsilon-\varepsilon^{p}
$$

The stress $\sigma$ is governed by the elastic constitutive equation (when $f<0$ ), and is calculated as

$$
\sigma=E \varepsilon^{e}=E\left(\varepsilon-\varepsilon^{p}\right)
$$

The phenomenological properties that were described in section 6.1 must be satisfied in the constitutive equations [68].

The stress cannot be greater in absolute value than $\sigma_{Y}$. Therefore, the stresses cannot exceed the closed interval $\left[-\sigma_{Y}, \sigma_{Y}\right]$ where $\sigma_{Y}$ is the yield stress. This condition is formally expressed as

$$
\mathbb{E}_{\sigma}=\sigma \in \mathbb{R}|f(\sigma):=| \sigma \mid-\sigma_{Y} \leq 0
$$

where $\sigma_{y}$ is the yield stress and $f$ is the yield function. If $|\sigma|$ is less than the flow stress, no change in $\varepsilon^{p}$ occurs, so

$$
\dot{\varepsilon}^{p}=0, \quad \text { if } \quad f(\sigma)<0
$$

Plastic strain occurs when the yield criterion is satisfied; $f(\sigma):=|\sigma|-\sigma_{Y}=0$. Introducing a parameter $\dot{\lambda} \geq 0$ as $\dot{\lambda}=\left|\dot{\varepsilon}^{p}\right|$, the plastic slip can be expressed as

$$
\begin{gathered}
\dot{\varepsilon}^{p}=\dot{\lambda}, \quad \text { if } \quad \sigma=\sigma_{Y} \\
\dot{\varepsilon}^{p}=-\dot{\lambda}, \quad \text { if } \quad \sigma=-\sigma_{Y}
\end{gathered}
$$

This may be written as Equation 6.8, and is known as the flow rule.

$$
\dot{\varepsilon}^{p}=\dot{\lambda} \operatorname{sign}(\sigma)
$$


The sign function is defined as

$$
\operatorname{sign}(\sigma)= \begin{cases}+1 & \text { if } \sigma>0 \\ -1 & \text { if } \sigma<0\end{cases}
$$

The boundary of the elastic range is called the yield surface, and is defined as

$$
\partial \mathbb{E}_{\sigma}=\left\{\sigma \in \mathbb{R}|f(\sigma):=| \sigma \mid-\sigma_{Y}=0\right\}
$$

From the above discussion it follows that $\sigma$ must be valid $(\sigma \in \mathbb{E})$ and $\dot{\lambda}$ must be non-negative $[72,73]$

$$
\dot{\lambda} \geq 0, f(\sigma) \leq 0
$$

Also if

$$
\begin{aligned}
& f(\sigma)<0 \Rightarrow \dot{\lambda}=0, \\
& \dot{\lambda}>0 \Rightarrow f(\sigma)=0,
\end{aligned}
$$

which implies

$$
\dot{\lambda} f(\sigma)=0
$$

Conditions 6.11 and 6.13 are the Kuhn-Tucker conditions. Now consider a Taylor expansion of the function

$$
\hat{f}(t+\Delta t)=\hat{f}(t)+\dot{\hat{f}}(t) \Delta t+(O)(\Delta t)
$$

where $\dot{\hat{f}}(t)=\frac{\partial}{\partial t} \hat{f}(t)$ and $(O)(\Delta t)$ represents the Taylor series terms that tend to zero faster than $\Delta t$. Since the assumption $\hat{f}(t)=0$, it has $\dot{\hat{f}}(t) \leq 0$. Therefore

$$
\begin{aligned}
& \dot{f}<0 \Rightarrow \dot{\lambda}=0 \\
& \dot{\lambda}>0 \Rightarrow \dot{f}=0
\end{aligned}
$$


which implies that

$$
\dot{\lambda} \dot{f}(\sigma)=0
$$

The 1-D model for rate independent perfect elasto-plasticity [72] is shown as follows in Box 6.3.

\section{Box. 6.3.1: 1-D Model for rate independent perfect elasto-} plasticity

1. Additive strain split: $\varepsilon=\varepsilon^{e}+\varepsilon^{p}$ [eq.6.1]

2. Elastic constitutive law: $\sigma=E \varepsilon^{e}$ [eq.6.3]

3. Yield criterion: $f(\sigma):=|\sigma|-\sigma_{Y} \leq 0$ [eq.6.4]

4. Flow rule: $\dot{\varepsilon}^{p}=\dot{\lambda} \operatorname{sign}(\sigma)$ [eq.6.8]

5. Kuhn-Tucker (loading/unloading) conditions: $\dot{\lambda} \geq 0, f(\sigma) \leq$ $0, \dot{\lambda} f(\sigma)=0$ [eq.6.11, 6.13]

6. Consistency condition $\dot{\lambda} \dot{f}(\sigma)=0$, Equation 6.14

\subsection{Strain Hardening}

Strain hardening occurs when a solid is plastically deformed, then unloaded, and then re-loaded so as to induce further plastic flow. It will be found that its resistance to plastic flow would have increased; its yield point/elastic limit increases. For a finite element material model, there are two ways for modelling the strain hardening:

1. Isotropic Hardening: plastically deform a solid, then unload it, then reload it again. It will be found that the yield stress/elastic limit would have increased in comparison to the first cycle. In other words, if something was loaded in tension past its yield point, then unloaded, and then loaded in compression, it will not yield in compression until it reaches the level past yield achieved when loading in tension.

2. Kinematic Hardening: correctly model cyclic behaviour and the Bauschinger effect, since the material softens in compression.

In the scope of this research, only the isotropic hardening is considered. 


\section{Isotropic Hardening}

The mathematical representation for the yield criterion in isotropic hardening is:

$$
f(\sigma, \alpha)=|\sigma|-\left[\sigma_{Y}+H \alpha\right] \leq 0
$$

where $\alpha$ is a hardening parameter (internal variable) that describes the evolution of the elastic domain, and $\alpha>0$. There are several ways to represent $\alpha$. The most widely used approach represents strain and work hardening respectively as

$$
\dot{\alpha}=\left|\dot{\varepsilon}^{p}\right|, \text { and } \dot{\alpha}=\sigma\left|\dot{\varepsilon}^{p}\right|
$$

The 1-D Model for rate independent isotropic hardening elasto-plasticity is presented in Box 6.4.

\section{Box. 6.4.1: 1-D Model for rate independent isotropic hardening}

\section{elasto-plasticity}

1. Additive strain split: $\varepsilon=\varepsilon^{e}+\varepsilon^{p}$ [eq.6.1]

2. Elastic constitutive law: $\sigma=E \varepsilon^{e}$ [eq.6.3]

3. Yield criterion: $f(\sigma, \alpha)=|\sigma|-\left[\sigma_{Y}+H \alpha\right] \leq 0 \quad$ [eq.6.19]

4. Flow rule and hardening Law: $\dot{\varepsilon}^{p}=\dot{\lambda} \operatorname{sign}[\sigma], \dot{\alpha}=\left|\dot{\varepsilon}^{p}\right|$ [eq.6.8,6.20]

5. Kuhn-Tucker loading/unloading conditions: $\dot{\lambda} \geq 0, f(\sigma, \alpha) \leq$ $0, \dot{\lambda} f(\sigma, \alpha)=0$ [eq.6.11, 6.13]

6. Consistency condition, Equation 6.14

\section{Tangent Elasto-plastic Modulus}

The tangent modulus $\hat{C}=d \sigma / d \varepsilon$ is obtained by explicitly solving for the parameter $\dot{\lambda}$. The consistency condition is used for this purpose.

$$
\dot{f}(\sigma, \alpha)=\frac{\partial f(\sigma, \alpha)}{\partial \sigma} \dot{\sigma}+\frac{\partial f(\sigma, \alpha)}{\partial \alpha} \dot{\alpha}
$$


taking the partial derivative with respect to $\sigma$ and $\alpha$ gives

$$
\begin{array}{r}
\frac{\partial f(\sigma, \alpha)}{\partial \sigma} \dot{\sigma}=|\dot{\sigma}|=\left|E \dot{\varepsilon}^{e}\right|=\left|E\left(\dot{\varepsilon}-\dot{\varepsilon}^{p}\right)\right|=\operatorname{sign}[\sigma] E\left(\dot{\varepsilon}-\dot{\varepsilon}^{p}\right) \\
\frac{\partial f(\sigma, \alpha)}{\partial \alpha} \dot{\alpha}=-H \dot{\alpha}
\end{array}
$$

From Equation 6.21, the tangent elasto-plastic modulus is:

$$
\dot{f}(\sigma, \alpha)=\operatorname{sign}[\sigma] E\left(\dot{\varepsilon}-\dot{\varepsilon}^{p}\right)-H \dot{\alpha}=\operatorname{sign}[\sigma] E\left(\dot{\varepsilon}-\dot{\varepsilon}^{p}\right)-H \dot{\varepsilon}^{p}
$$

From Equation 6.20,

$$
\begin{gathered}
\dot{f}(\sigma, \alpha)=\operatorname{sign}[\sigma] E\left(\dot{\varepsilon}-\dot{\varepsilon}^{p}\right)-\dot{\varepsilon}^{p} H \\
\rightarrow \operatorname{sign}[\sigma]\left(E \dot{\varepsilon}-\dot{\varepsilon}^{p}(E+H)\right)
\end{gathered}
$$

By using the flow rule, Equation 6.8: $\dot{\lambda}=\dot{\varepsilon}^{p} \operatorname{sign}[\sigma]$

$$
\dot{f}(\sigma, \alpha)=\operatorname{sign}[\sigma] E \dot{\varepsilon}-\dot{\lambda}(E+H)
$$

By using the consistency condition that $\dot{f}(\sigma, \alpha)=0$, then solving for $\dot{\lambda}$, gives

$$
\dot{\lambda}=\frac{\operatorname{sign}[\sigma] E}{E+H} \dot{\varepsilon}
$$

Hence, the following rate form is obtained

$$
\dot{\sigma}= \begin{cases}E \dot{\varepsilon} & \text { if } f<0 \\ \frac{E H}{E+H} & \text { if } f=0\end{cases}
$$

\subsection{Incremental $1 \mathrm{D}$ rate independent plasticity}

Assuming equal time steps $\Delta t$, the discrete time instances $t_{0}, t_{1}, t_{2}, \ldots$ are indicated by subscripts; generally denoted as equation 6.30 , so the strain increment is 
denoted as $\varepsilon_{n+1}$ :

$$
\begin{gathered}
t_{n+1}=t_{n}+\Delta t \\
\varepsilon_{n+1}=\varepsilon_{n}+\Delta \varepsilon_{n}
\end{gathered}
$$

The backward Euler method is used to transform the equations to incremental form at time instant $t_{n+1}$. The algebraic equations become [72];

1. Flow rule

$$
\frac{\varepsilon_{n+1}^{p}-\varepsilon_{n}^{p}}{\Delta t}=\frac{\Delta \lambda}{\Delta t} \operatorname{sign}\left(\sigma_{n+1}\right)
$$

2. Isotropic Hardening Law

$$
\frac{\alpha_{n+1}-\alpha_{n}}{\Delta t}=\frac{\Delta \lambda}{\Delta t}
$$

3. Yield function

$$
f\left(\sigma_{n+1}, \alpha_{n+1}\right)=\left|\sigma_{n+1}\right|-\left(\sigma_{y}+H \alpha_{n+1}\right)
$$

4. Complementary conditions

$$
\Delta \lambda \geq 0, \quad f_{n+1} \leq 0, \quad \Delta \lambda f_{n+1}=0
$$

Since there are two inequalities that do not give a direct solution, operator splitting is applied to get rid of them: predictor and corrector.

\subsubsection{Elastic step (Predictor Step)}

The elastic trial step is first done by freezing the plastic flow: $\Delta \lambda=0$. This state is admissible only when the incremental process is elastic.

$$
\begin{aligned}
\varepsilon_{n+1}^{p, \text { trial }} & =\varepsilon_{n}^{p} \\
\sigma_{n+1}^{\text {trial }} & =E\left(\varepsilon_{n+1}-\varepsilon_{n}^{p}\right)=E\left(\left(\varepsilon_{n+1}-\varepsilon_{n}\right)+\left(\varepsilon_{n}-\varepsilon_{n}^{p}\right)\right)=\sigma_{n}+E \Delta \varepsilon_{n}
\end{aligned}
$$




$$
\begin{aligned}
\alpha_{n+1}^{\text {trial }} & =\alpha_{n} \\
f_{n+1}^{\text {trial }} & =\left|\sigma_{n+1}^{\text {trial }}\right|-\left(\sigma_{Y}+H \alpha_{n}\right)
\end{aligned}
$$

\subsubsection{Plastic step (Corrector Step)}

If $f_{n+1}^{\text {trial }} \leq 0$ the trial state is admissible. So, $\varepsilon_{n+1}^{p}=\varepsilon_{n}^{p}, \alpha_{n+1}=\alpha_{n}$ and $\sigma_{n+1}=$ $\sigma_{n+1}^{\text {trial }}$. However if $f_{n+1}^{\text {trial }}>0$ then the trial state is violating the complimentary condition that $f(\alpha, \sigma)<0$ and $\Delta \lambda>0$ is required. In other words if $f_{n+1}^{\text {trial }} \leq 0$ then this is an elastic step and $\Delta \lambda=0$. If $f_{n+1}^{\text {trial }}>0$, then this is a plastic step and $\Delta \lambda>0$, and a return mapping algorithm can be implemented to accommodate for that.

\subsubsection{Return Mapping}

As mentioned earlier, the return mapping algorithm is implemented because the trial stress lies outside of the yield surface. The return mapping algorithm brings the stress that lies outside the plastically admissible domain back to the yield surface. Figure 6.2 is a visual representation of the return mapping scheme [68], where Figure $6.2 \mathrm{a}$ shows that the elastic predictor takes $\sigma_{n}$ to $\sigma_{n+1}^{\text {trial }}$, and the plastic corrector will bring back $\sigma_{n+1}^{\text {trial }}$ to $\sigma_{n+1}$ the new (updated) yield surface (graphically shown as $\phi$, otherwise presented in this thesis as $f$ ). In Figure 6.2b, the predictor-corrector is similar. However since there is no hardening (perfect plasticity), the plastic corrector will place $\sigma_{n+1}$ at the initial yield surface. The first of this algorithm was presented by Wilkins1984 in the "radial return method" [Wilkins1984].

An expression for $\sigma_{n+1}$ in terms of $\sigma_{n+1}^{\text {trial }}$ must be written

$$
\begin{aligned}
\sigma_{n+1} & =E\left(\varepsilon_{n+1}-\varepsilon_{n+1}^{p}\right) \\
& =E\left(\varepsilon_{n+1}-\varepsilon_{n}^{p}\right)-E\left(\varepsilon_{n+1}^{p}-\varepsilon_{n}^{p}\right) \\
& =\sigma_{n+1}^{\text {trial }}-E \Delta \lambda \operatorname{sign}\left(\sigma_{n+1}\right)
\end{aligned}
$$




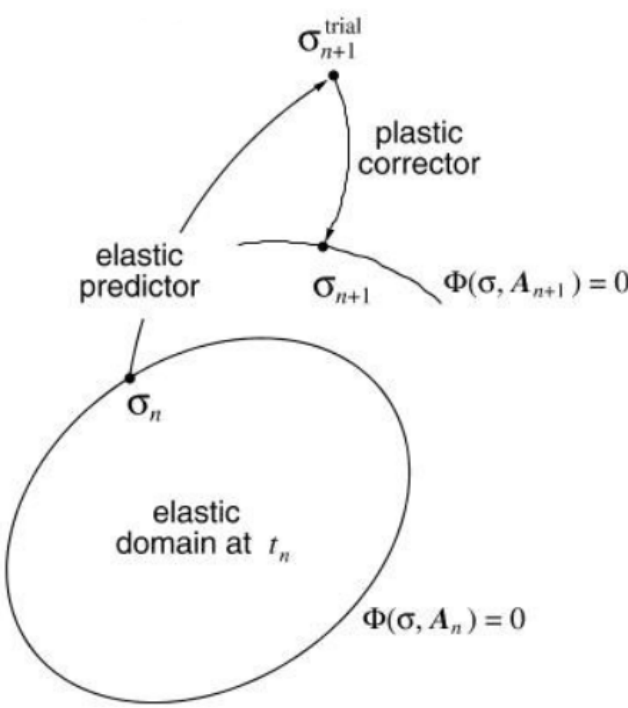

(a)

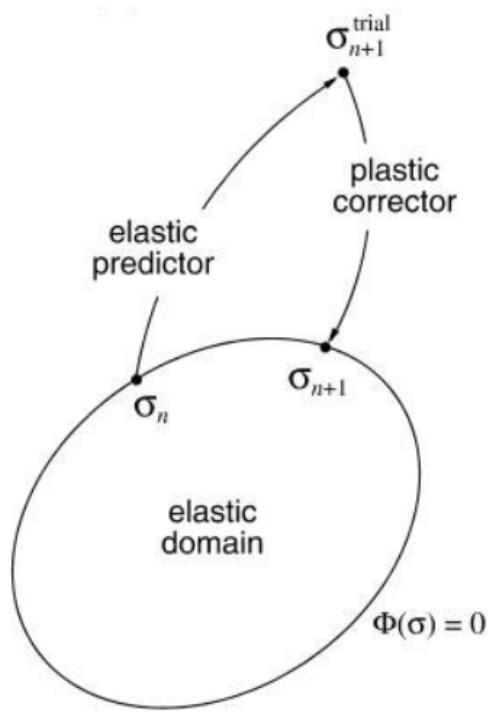

(b)

Figure 6.2: Return mapping scheme (a) with hardening (b) no hardening [68]

Applying this expression in incremental Equations 6.35 - 6.45 gives

$$
\begin{aligned}
\sigma_{n+1} & =\sigma_{n+1}^{\text {trial }}-E \Delta \lambda \operatorname{sign}\left(\sigma_{n+1}\right) \\
\varepsilon_{n+1}^{p} & =\varepsilon_{n}^{p}+\Delta \lambda \operatorname{sign}\left(\sigma_{n+1}\right) \\
\alpha_{n+1} & =\alpha_{n}+\Delta \lambda \\
f_{n+1} & =\left|\sigma_{n+1}^{\text {trial }}\right|-\left(\sigma_{Y}+H \alpha_{n+1}\right)=0
\end{aligned}
$$

The sign of the stress is the sign of the trial stress [72]

$$
\begin{aligned}
\sigma_{n+1} & =\left|\sigma_{n+1}\right| \operatorname{sign}\left(\sigma_{n+1}\right)=\left|\sigma_{n+1}^{\text {trial }}\right| \operatorname{sign}\left(\sigma_{n+1}^{\text {trial }}\right)-E \Delta \lambda \operatorname{sign}\left(\sigma_{n+1}\right) \\
& \Rightarrow\left(\left|\sigma_{n+1}\right|+\Delta \lambda E\right) \operatorname{sign}\left(\sigma_{n+1}\right)=\left|\sigma_{n+1}^{\text {trial }}\right| \operatorname{sign}\left(\sigma_{n+1}^{\text {trial }}\right) \\
& \Rightarrow \operatorname{sign}\left(\sigma_{n+1}\right)=\operatorname{sign}\left(\sigma_{n+1}^{\text {trial }}\right) \\
& \Rightarrow\left(\left|\sigma_{n+1}\right|+\Delta \lambda E\right)=\left|\sigma_{n+1}^{\text {trial }}\right|
\end{aligned}
$$




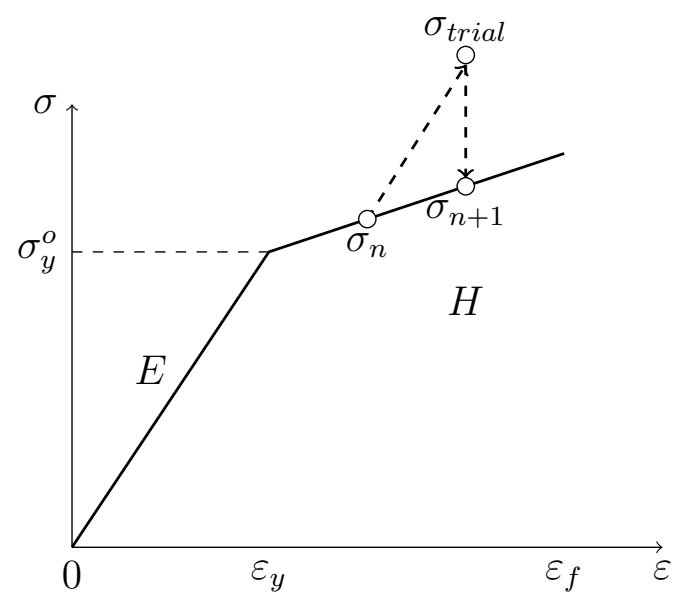

Figure 6.3: Return Mapping with hardening

Therefore [72]

$$
\begin{aligned}
f_{n+1} & =\left|\sigma_{n+1}\right|-\left(\sigma_{Y}+H \alpha_{n+1}\right) \\
& =\left|\sigma_{n+1}^{\text {trial }}\right|-\Delta \lambda-\left(\sigma_{Y}+H \alpha_{n+1}\right) \\
& =\left|\sigma_{n+1}^{\text {trial }}\right|-\Delta \lambda-\left(\sigma_{Y}+H \alpha_{n}\right)-H\left(\alpha_{n+1}-\alpha_{n}\right) \\
& =f_{n}^{\text {trial }}-\Delta \lambda(E+H)=0 \\
& \Rightarrow \Delta \lambda=\frac{F_{n+1}^{\text {trial }}}{E+H}
\end{aligned}
$$

Hence [72]

$$
\begin{aligned}
& \sigma_{n+1}=\sigma_{n+1}^{\text {trial }}-E \Delta \lambda \operatorname{sign}\left(\sigma_{n+1}^{\text {trial }}\right) \\
& \varepsilon_{n+1}^{p}=\varepsilon_{n}^{p}+\Delta \lambda \operatorname{sign}\left(\sigma_{n+1}^{\text {trial }}\right) \\
& \alpha_{n+1}=\alpha_{n}+\Delta \lambda \\
& f_{n+1}=0
\end{aligned}
$$

Another visual representation of the return mapping algorithm is shown in Figure 6.3 where from $\sigma_{n}$ to $\sigma_{\text {trial }}$ is the predictor step, and then $\sigma_{\text {trial }}$ to $\sigma_{n+1}$ is the corrector (plastic) step.

The return mapping algorithm is summarised in Box 6.5.3 [72]. 


\section{Box. 6.5.1: Incremental 1D Return-mapping (predictor-} corrector) plasticity

1. Evaluate elastic predictor:

$$
\sigma_{n+1}^{\text {trial }}=\sigma_{n}+E \Delta \varepsilon_{n}[E q .6 .36]
$$

2. Discrete version of Khun-Tucker conditions:

$$
f_{n+1}^{\text {trial }}=\left|\sigma_{n+1}^{\text {trial }}\right|-\sigma_{Y}+H \alpha_{n} \quad[\text { eq. 6.45] }
$$

3. If $f_{n+1}^{\text {trial }} \leq 0 \rightarrow$ Elastic state
(a) $(\bullet)_{n+1}=(\bullet)_{n+1}^{\text {trial }}$
(b) Exit

Else $\rightarrow$ Plastic corrector
(a) $\Delta \lambda=\frac{F_{n+1}^{\text {trial }}}{E+H}$
(b) $\sigma_{n+1}=\sigma_{n+1}^{\text {trial }}-E \Delta \lambda \operatorname{sign}\left(\sigma_{n+1}\right)[E q$. 6.42]
(c) $\varepsilon_{n+1}^{p}=\varepsilon_{n}^{p}+\Delta \lambda \operatorname{sign}\left(\sigma_{n+1}\right)[E q .6 .43]$
(d) $\alpha_{n+1}=\alpha_{n}+\Delta \lambda[E q$. 6.44]

4. Repeat steps

\subsection{Application of AEM to elasto-plasticity}

Equations [6.42 - 6.44] are generally nonlinear and must be solved using some iterative procedures [68]. The common iterative procedure used in solving the return mapping equations is the Newton-Raphson method. The motivation of using this method is due to its quadratic rate of convergence [68]. The method is explained in the following section.

\subsubsection{Newton Raphson Method}

As mentioned, the Newton Raphson method is an efficient nonlinear equation solution scheme with a quadratic convergence. For the infinitesimal incremental boundary value problem, given the internal variables $\alpha_{n}$ at time $t_{n}$ such that

$$
\int_{\Omega}\left[\hat{\sigma}\left(\alpha_{n}, \nabla^{s} u_{n+1}\right): \nabla^{s} \eta-b_{n+1} \cdot \eta\right] d v-\int_{\partial \Omega_{t}} t_{n+1} \cdot \eta d a=0
$$


where $b_{n+1}$ and $t_{n+1}$ are the body force and the surface traction fields prescribed at time instant $t_{n+1}$. Equation 6.59 can be reduced after a standard finite element discretisation. Let $u_{n+1}$ be the nodal displacement at time $t_{n+1}$, the incremental finite element equation becomes

$$
r\left(u_{n+1}\right) \equiv f_{\text {int }}\left(u_{n+1}\right)-f_{n+1}^{e x t}=0
$$

where $f^{i n t}\left(u_{n+1}\right)$ and $f_{n+1}^{e x t}$ are assembled from the element vectors in Equations 6.61 and 6.62. The nonlinearity in Equation 6.60 is from the incremental constitutive function that takes part in the definition of the element internal force vector [68].

$$
\begin{aligned}
& f_{(e)}^{\text {int }}=\int_{\Omega^{(e)}} B^{T} \hat{\sigma}\left(\alpha_{n}, \varepsilon\left(u_{n+1}\right)\right) d v \\
& f_{(e)}^{e x t}=\int_{\Omega^{(e)}} N^{T} b_{n+1} d v+\int_{\partial \Omega^{(e)}} N^{T} t_{n+1} d a
\end{aligned}
$$

The loading is described by the body force and te surface traction fields at an instant $t_{n+1}$ by

$$
\begin{aligned}
b_{n+1} & =\lambda_{n+1} \tilde{b} \\
t_{n+1} & =\lambda_{n+1} \tilde{t}
\end{aligned}
$$

where $\lambda_{n+1}$ is the load factor at $t_{n+1}$ and $\tilde{b}$ and $\tilde{t}$ are prescribed constant fields in time [68]. The global external force reduces to

$$
f_{n+1}^{e x t}=\lambda_{n+1} \bar{f}^{e x t}
$$

where $\bar{f}^{e x t}$ is computed only once at the beginning of the incremental procedure for the element vectors as [68]

$$
\bar{f}_{(e)}^{e x t}=\int_{\Omega^{(e)}} N^{T} \tilde{b} d v+\int_{\partial \Omega_{t}^{(e)}} N^{T} \tilde{t} d a
$$


The typical iteration of the Newton-Raphson scheme consists of solving the linear(ised) system of equations for $\delta u^{(k)}[68]$

$$
K_{T} \delta u^{(k)}=-r^{(k-1)}
$$

where the the residual vector is

$$
r^{(k-1)} \equiv f^{i n t}\left(u_{n+1}^{(k-1)}\right)-f_{n+1}^{e x t}
$$

and $K_{T}$ is the global tangent stiffness matrix obtained from the applied element method. With the solution of $\delta u^{(k)}$, applying the Newton correction to the global displacement gives [68]

$$
u_{n+1}^{(k)}=u_{n+1}^{(k-1)}+\delta u^{(k)}
$$

and in terms of displacement increments [68]

$$
u_{n+1}^{(k)}=u_{n}+\Delta u^{(k)}
$$

where $\Delta u^{(k)}$ is the incremental displacement vector

$$
\Delta u^{(k)}=\Delta u^{(k-1)}+\delta u^{(k)}
$$

The Newton Raphson iterations are repeated until the following convergence criteria are satisfied

$$
\frac{\left|r^{(i)}\right|}{f_{n+1}^{e x t}} \leq \epsilon_{t o l}
$$

where $\epsilon_{t o l}$ is a small specified value for equilibrium convergence tolerance. The schematic representation of the Newton-Raphson iteration procedure is shown in Figure 6.4. 


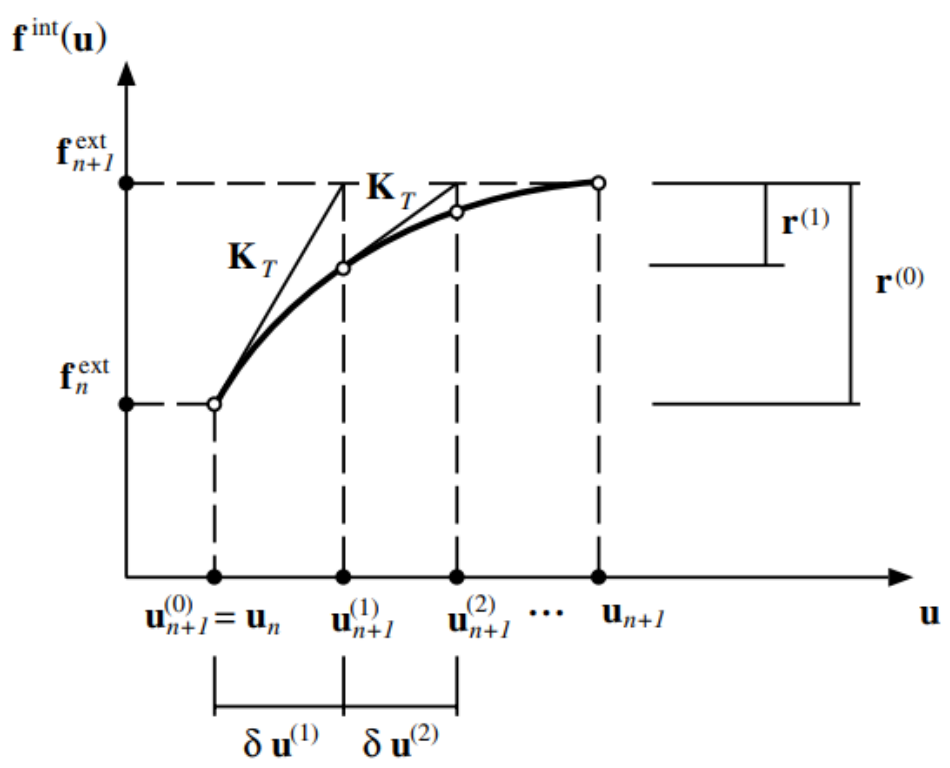

Figure 6.4: Newton Raphson iteration - graphical representation [68]

\subsection{Gaussian AEM in Hardening Elasto-plasticity}

The hardening elasto-plasticity is implemented into the Gaussian based AEM code. This section demonstrates different examples and implementations of behaviour of structures compared to FEM. For every load step, a Newton Raphson iteration begins where $\alpha$ and $\varepsilon$ (the plastic parameters) are updated and carried to the next Newton Raphson iteration, and to the next load step. This is what ensures that during unloading, if the stress had exceeded the yield stress limit, the plasticity effect would remain. Different beams are presented below, with loading-unloading-loading type mechanism, in order to view the behaviour of the force-displacement diagram. For all the examples presented, a maximum of 20 Newton Raphson iterations is set as the limit in case the convergence criteria is not met. Moreover, the tolerance for the convergence criteria is $10^{-3}$.

Comparison of Ansys results with Gaussian AEM with material nonlinearity was examined. The deflection from the ANSYS analysis at the free end of the beam was $0.0112 \mathrm{~m}$, and from Gaussian AEM was $0.0117 \mathrm{~m}$. The discrepancy is about $3 \mathrm{~mm}$. These results are considered accurate enough for using the Gaussian AEM. Therefore a detailed analysis of the cantilever beam using the Gaussian AEM is presented. 


\subsubsection{Cantilever beam}

Considering a cantilever beam with load incrementally applied at the free end. The stresses and beam deflection are shown as follows.

\section{Beam deflection}

The beam has 30 elements shown in Figure 6.5, with 10 Gaussian distributed springs. The maximum applied load on the cantilever is $1000 \mathrm{kN}$, and a total of 60 load increments for loading, unloading and loading again. The load displacement diagram of the free end of the beam is shown in Figure 6.6. The figure shows good accordance with expected behaviour from Figure 6.1, since the the stress increases linearly with the slope of the modulus of elasticity $E$, then after the stress yield limit, the slope changes with the value of the hardening. At unloading, the stress decreases with slope $E$. Loading again causes the stress to follow the linear path of $E$ and continue to a new yield stress limit where the path continues with $H$. The stress which the load will exceed the elastic limit is shown in Equation 6.73.

$$
\sigma=\frac{M y}{I}
$$

Since the maximum moment the beam encounters is at the fixed end with $M=$ $P l$, where $P$ is the applied force at the cantilever beam, and $l$ is the span of the beam. Substituting the moment in terms of the applied force $P$ gives

$$
P=\frac{\sigma I}{y l}
$$

Therefore, for the given cantilever beam with span $1 m$, cross section $b=h=0.15$, and yield stress $\sigma_{y}=250 \mathrm{MPa}$, the calculated yield force is $136.2 \mathrm{kN}$. Applying a force greater than the yield force will result in exceeding the elastic limit, and plasticity will be obtained. The deflection of the beam at different load steps is displayed in Figure 6.7. 


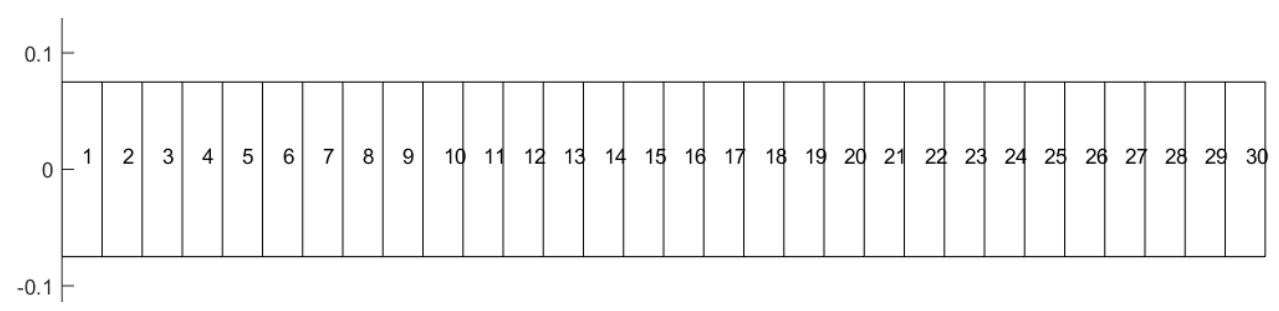

Figure 6.5: Cantilever beam elements

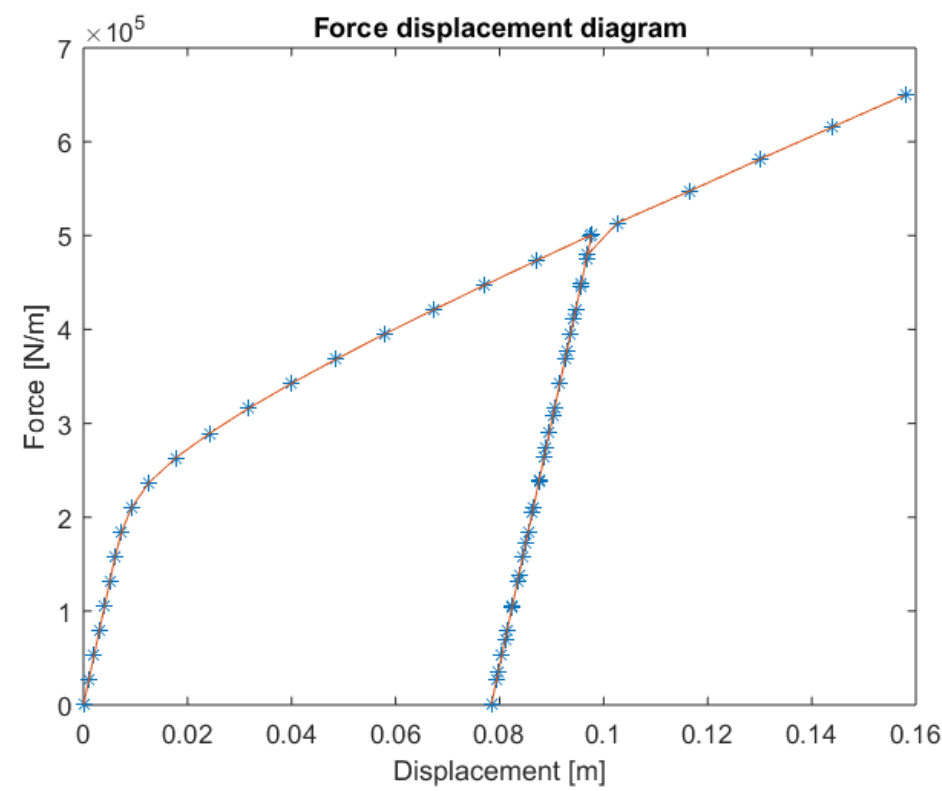

Figure 6.6: Load displacement diagram at free end

\section{Newton Raphson convergence}

Figure 6.8 shows the number of Newton Raphson iterations required at every load iteration. For cases of elasto-plasticity it can be seen that a maximum of four Newton Raphson iterations were required when a tolerance of $10^{-3}$ is used, shown in Figure 6.8a. Decreasing the tolerance to $10^{-5}$ resulted in more iterations, since the accuracy has improved. The number of iterations can be seen in Figure 6.8b. An important feature of using the Newton Raphson scheme is its quadratic convergence. Considering a point where the Newton Raphson iterations required is 5, the convergence plot is shown in Figure 6.9. The residual at every iteration is shown in Table 6.2. 


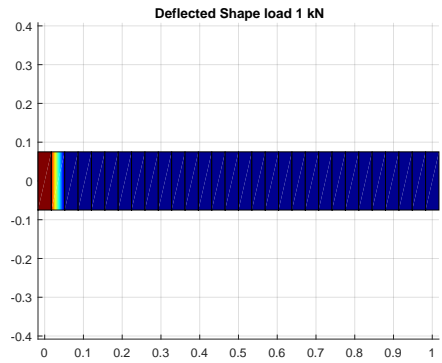

(a)

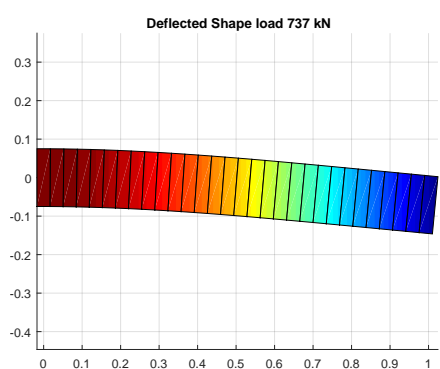

(d)

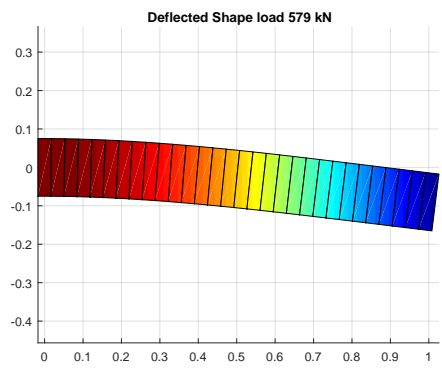

(g)

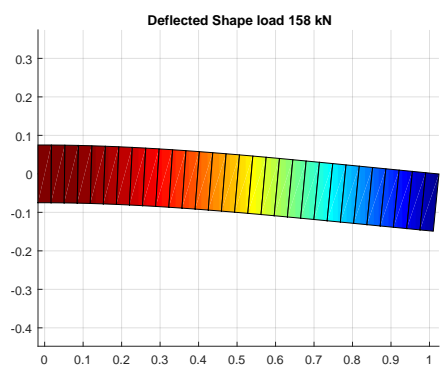

(j)

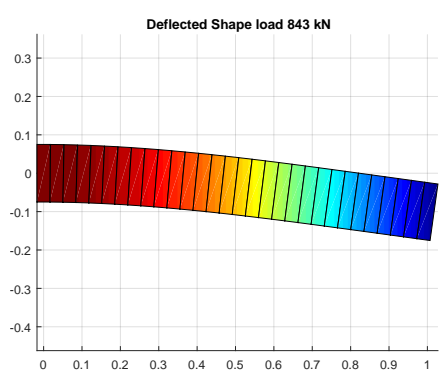

(m)

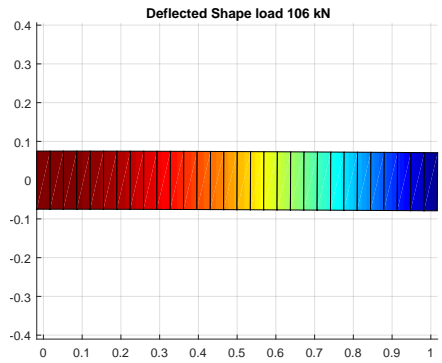

(b)

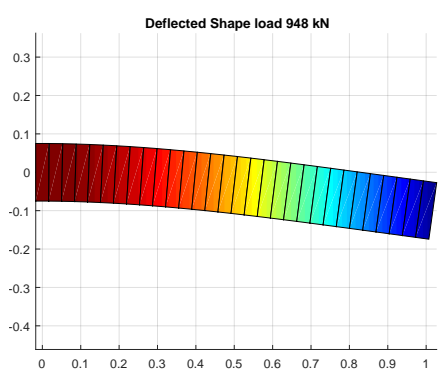

(e)

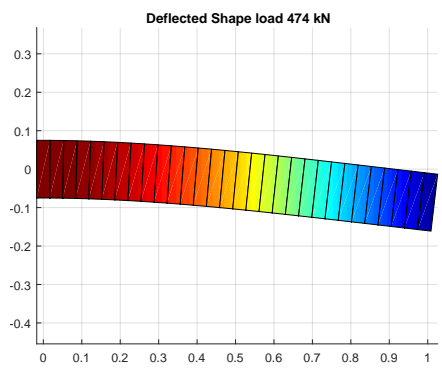

(h)

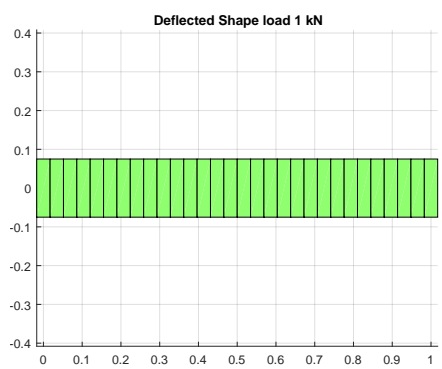

(k)

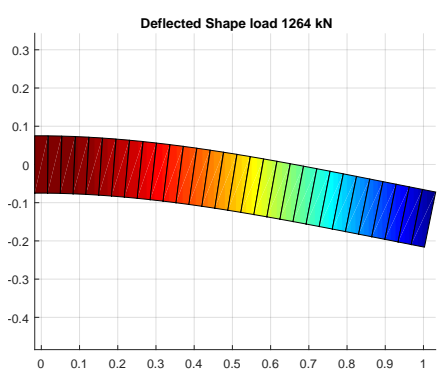

(n)

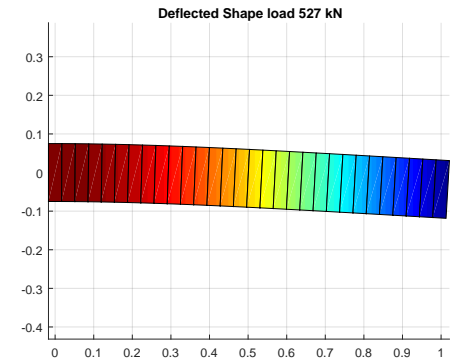

(c)

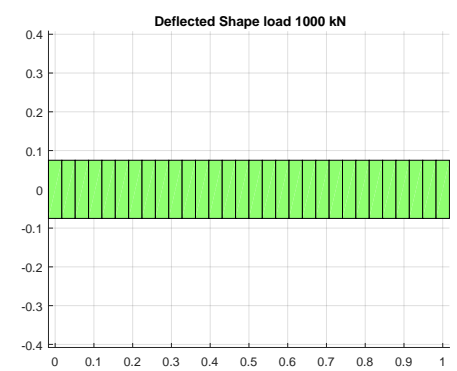

(f)

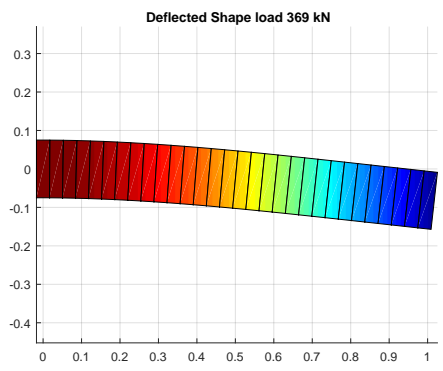

(i)

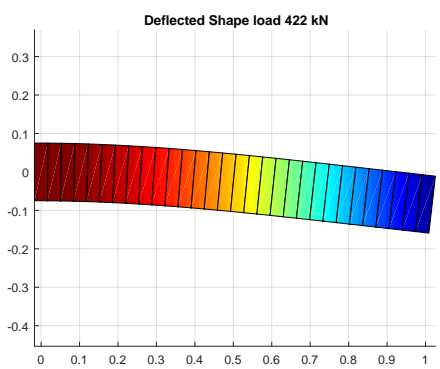

(1)

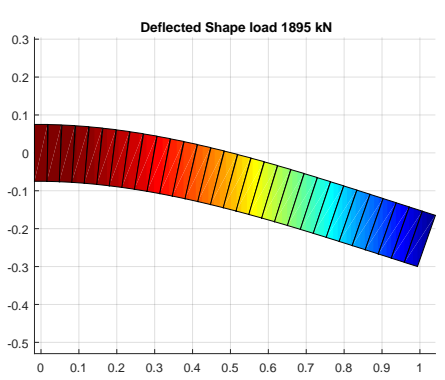

(o)

Figure 6.7: Beam deflection through loading-unloading-loading 


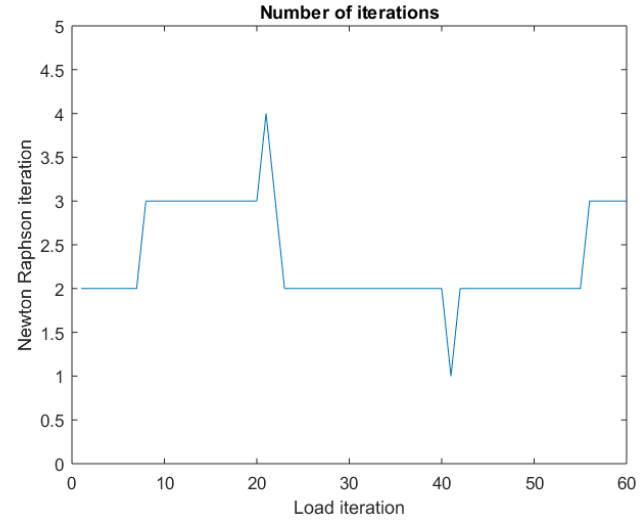

(a) tolerance $1 e-3$

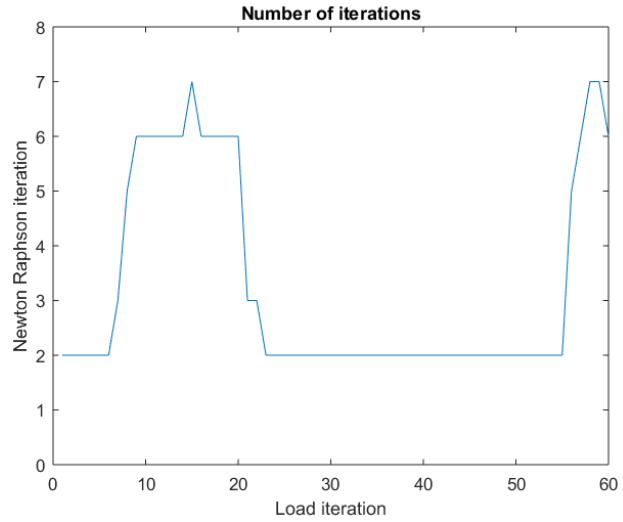

(b) tolerance $1 e-5$

Figure 6.8: Number of iterations required at every load step

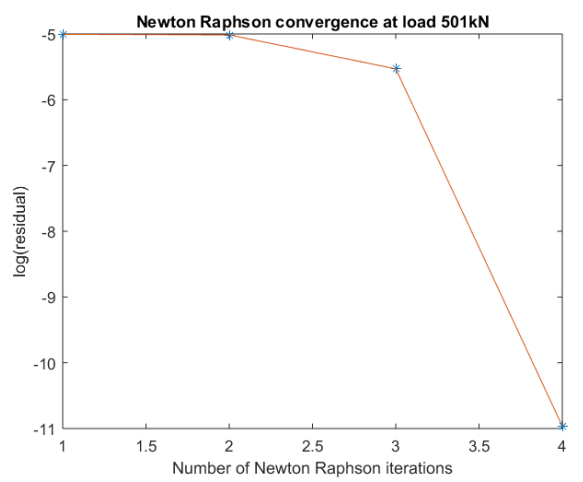

Table 6.1: Residual at each iteration

\begin{tabular}{c|cc}
\hline Iteration & Residual & $\log$ (residual) \\
\hline 1 & 0.006697 & -5.006 \\
2 & 0.006640 & -5.0145 \\
3 & 0.003961 & -5.5303 \\
4 & 0.000017 & -10.963
\end{tabular}

Figure 6.9: Newton Raphson convergence

\section{Normal and Shear Stresses}

For the cantilever beam with an applied load of $10^{7} N$, the normal stress is shown in Figure 6.11 and the shear stress of the beam is shown in Figure 6.12. From Figure 6.11 , the stress at 10 cross sections is shown. It is clear that the last 2 cross sections were linear, and the rest display the elasto-plastic behaviour. Also, the values at the top and bottom fibres at each cross-section are the same. The stress at the neutral axis is zero for all cross-sections. 


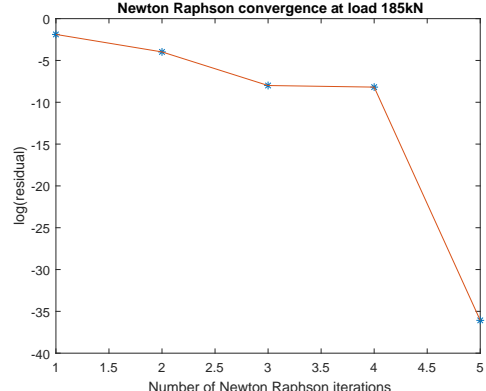

(a) $185 \mathrm{kN}$

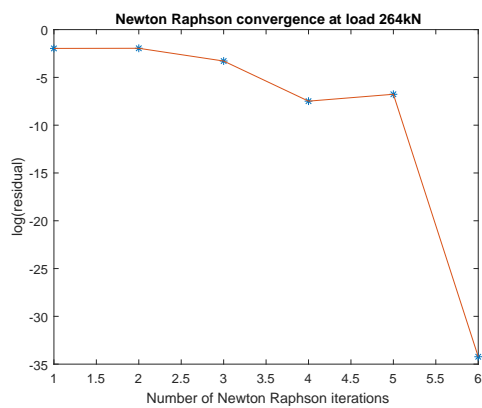

(d) $264 \mathrm{kN}$

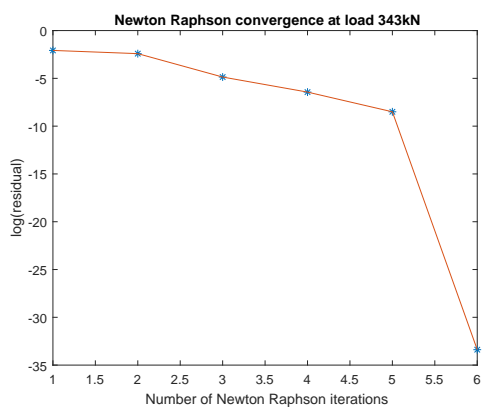

(g) $343 \mathrm{kN}$

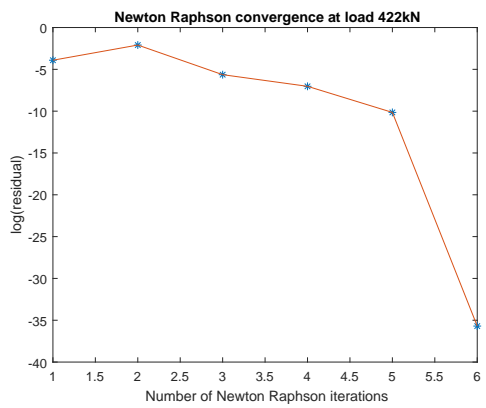

(j) $422 \mathrm{kN}$

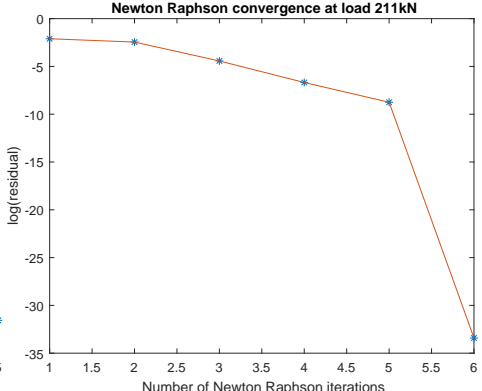

(b) $211 \mathrm{kN}$

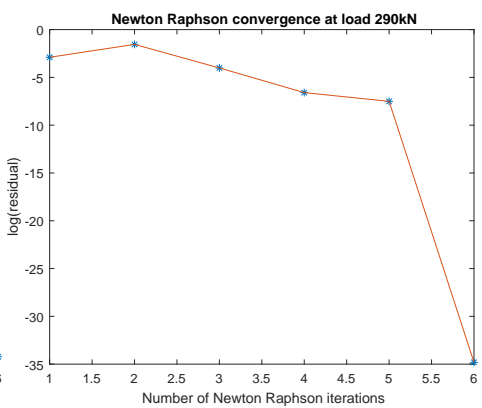

(e) $290 \mathrm{kN}$

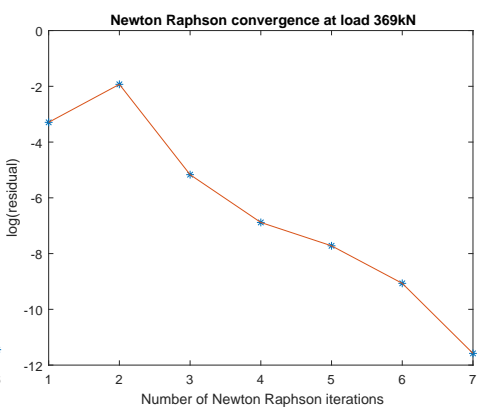

(h) $369 \mathrm{kN}$

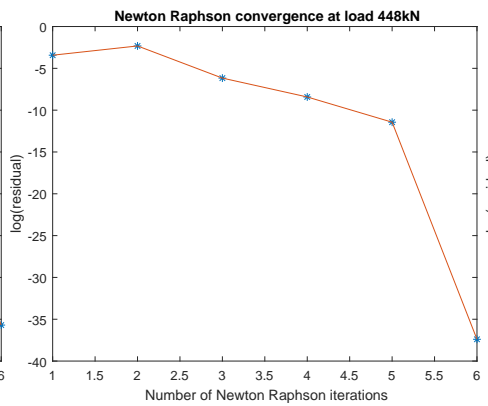

(k) $448 \mathrm{kN}$

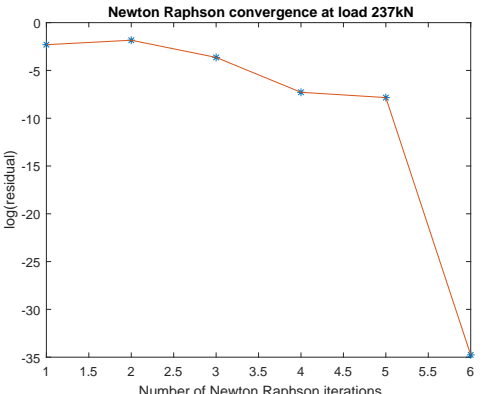

(c) $237 \mathrm{kN}$

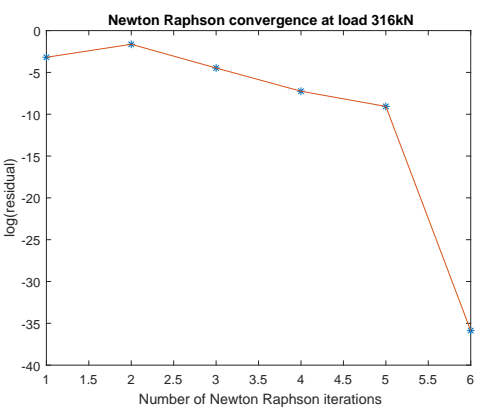

(f) $316 \mathrm{kN}$

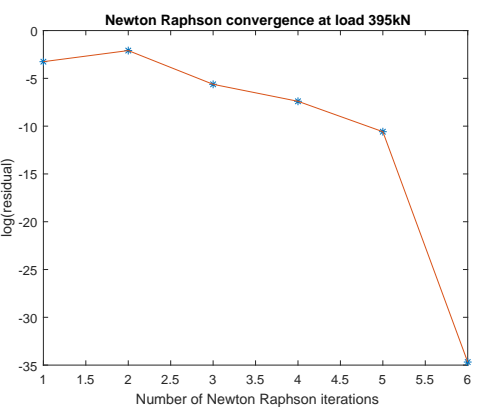

(i) $395 \mathrm{kN}$

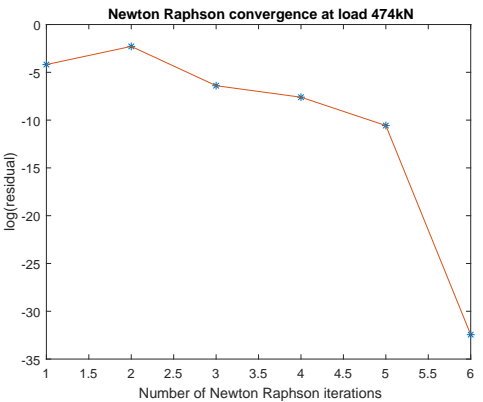

(l) $474 \mathrm{kN}$

Figure 6.10: Convergence at different load steps for tolerance $10^{-5}$ 


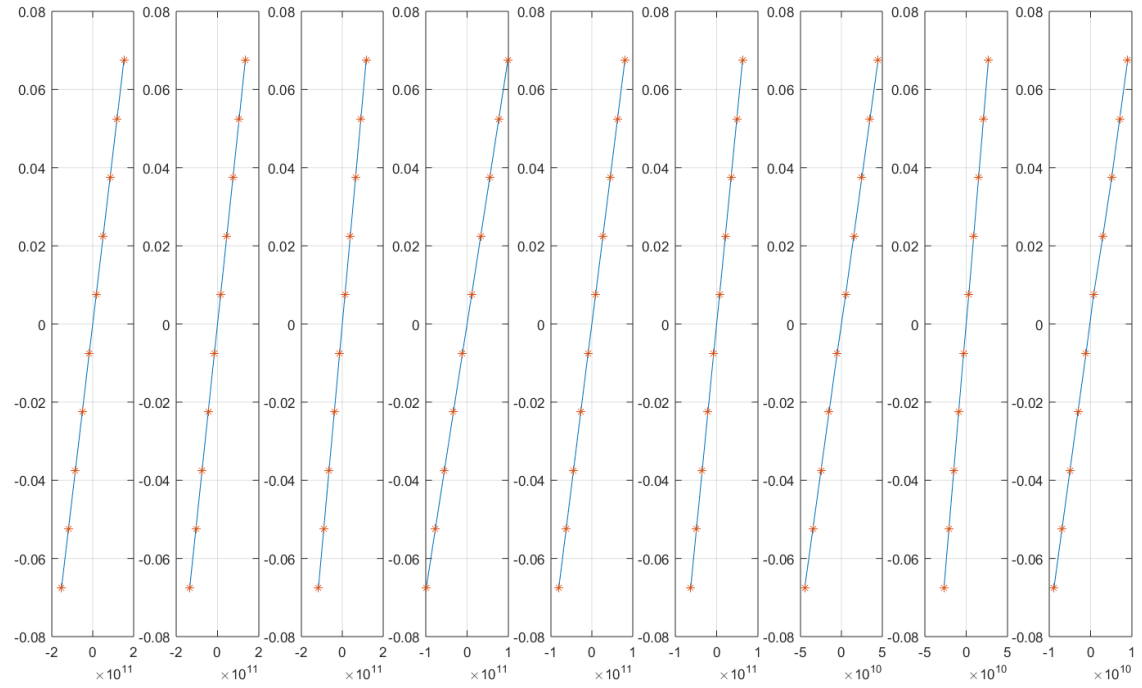

Figure 6.11: Normal stress for cantilever beam

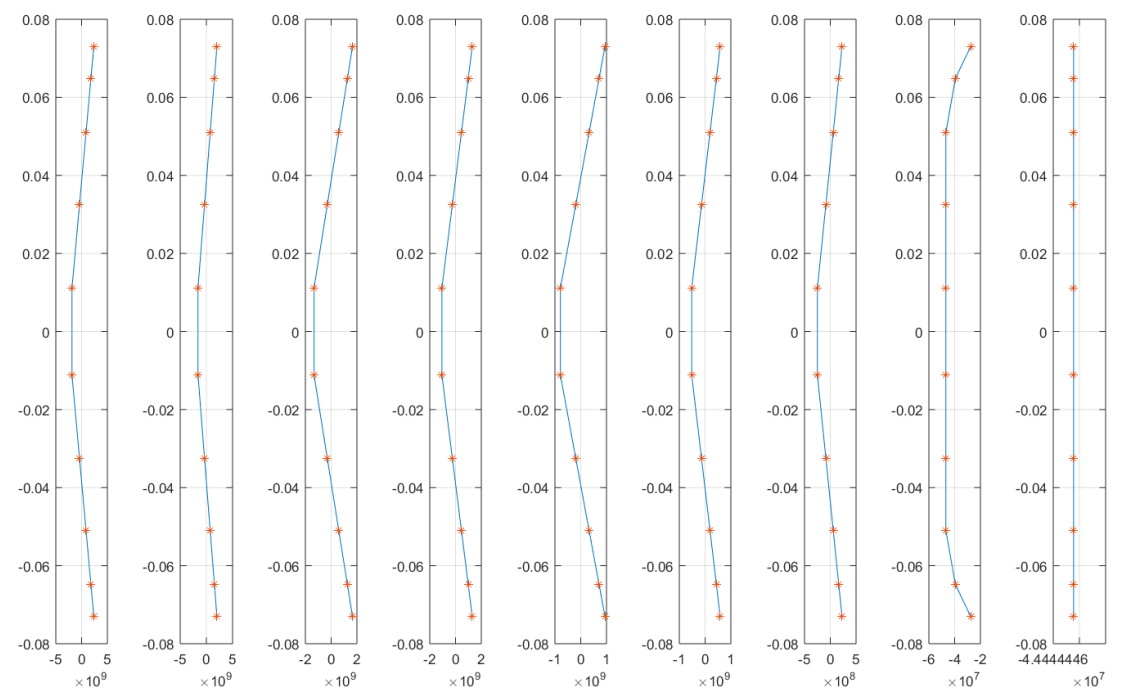

Figure 6.12: Shear stress for cantilever beam 


\subsection{Adaptive Gaussian Springs}

Using the Gaussian quadrature for the springs distribution, and the plasticity algorithm for calculating the stresses in the springs, an adaptive method for springs distribution can be implemented. In each iteration, the stress in each spring is calculated and determined whether it is in the elastic or the plastic stage. If all springs between two elements are elastic, then the initial number of springs will be changed to only two springs between elements, since only two points are required to model the linear behaviour of the elastic region, displayed in Figure 6.13. Hence, assuming an initial number of 10 springs between a pair of elements, after the first Newton Raphson iteration, if all springs are deemed to be elastic, then the total number of springs per pair of elements is reduced from 10 springs to 2 springs.

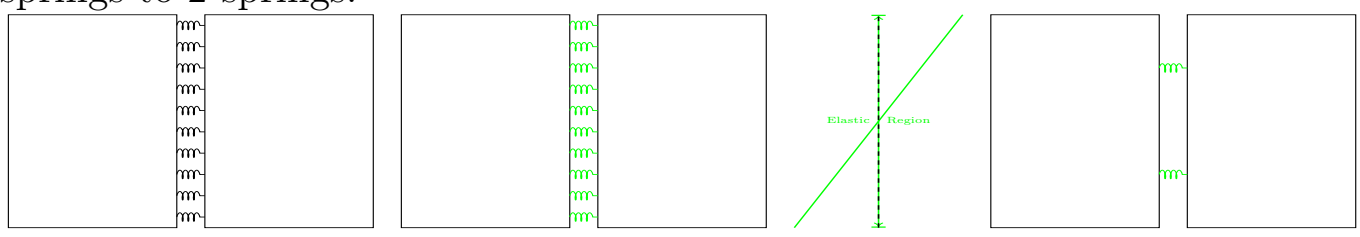

Figure 6.13: Adaptive Elastic Springs

However, if plasticity is observed in springs between a pair of elements, then the normal stress is split into 3 regions, plastic at the top and bottom, and elastic in the middle of the cross-section, as displayed in Figure 6.14. Since at least two springs are required for each region, then instead of having a total of 10 springs, 6 springs are required for elasto-plastic cases.
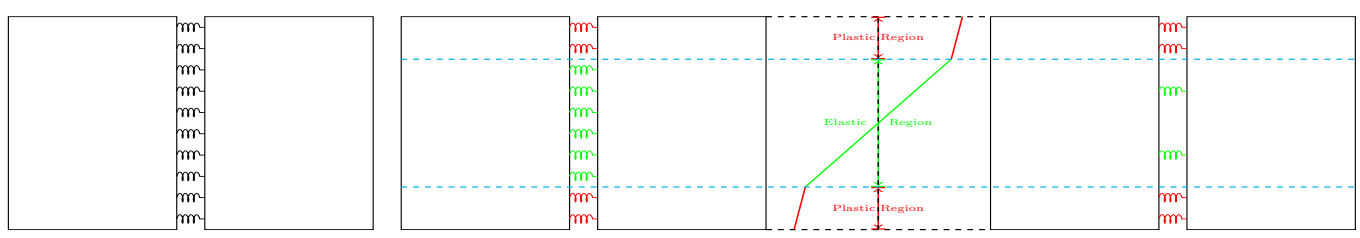

Figure 6.14: Adaptive Elasto-plastic Springs

An algorithm procedure for the beam deflection analysis would first entail an initial Newton Raphson iteration, where all spring stresses and strains are preallocated as zero. After the first iteration, the deflection vector is obtained and at the next iteration the stresses are recalculated. Depending on the yield criteria the springs are determined as belonging in the elastic or in the plastic region. Transition points at which exactly the change in regions occur are determined, 
and then the new spring locations are distributed.

The beam is analysed for the first Newton Raphson iteration, and the stresses in the springs are calculated. Based on the springs elasticity - whether they were elastic or plastic, the new springs will be placed between calculated transition points. The transition points are the points calculated where the stress changes from the elastic to plastic region, and vice versa.

Considering a cantilever beam fixed on the left end as displayed in Figure 6.17, and loaded on the right end. The spring distribution is resolved from the adaptive Gaussian Springs based Applied Element Method proposed. Initially there are 10 springs between each element, after determining the plasticity conditions of the springs, the new springs will be allocated. Assuming a small load was applied where all the springs remain in the elastic region, then all the springs between elements will be reduced from 10 springs per pair of elements, to two springs. While in the case of an elasto-plastic condition, where the elements closest to the fixed end will have higher stresses than the elements closer to the free end, it is expected that some plasticity will appear. So the elements with plasticity will have a total of 6 springs, while elements that are totally elastic will have 2 springs per element. In a more global effect, consider a beam with 10 elements, 10 springs between each element, the initial number of springs in total is $10 \times 9=90$ springs. After reduction, and in the case that all elements are elastic, the total number of springs becomes $2 \times 9=18$ springs.

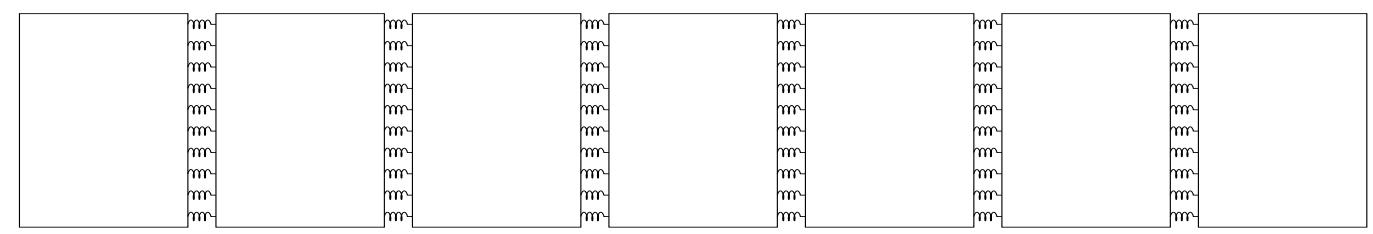

Figure 6.15: Representation of a cantilever with initial of 10 springs
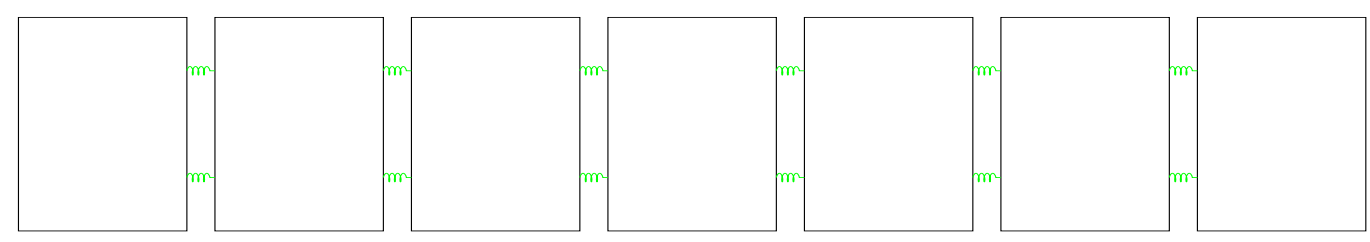

Figure 6.16: Representation of a cantilever beam with all elastic springs 

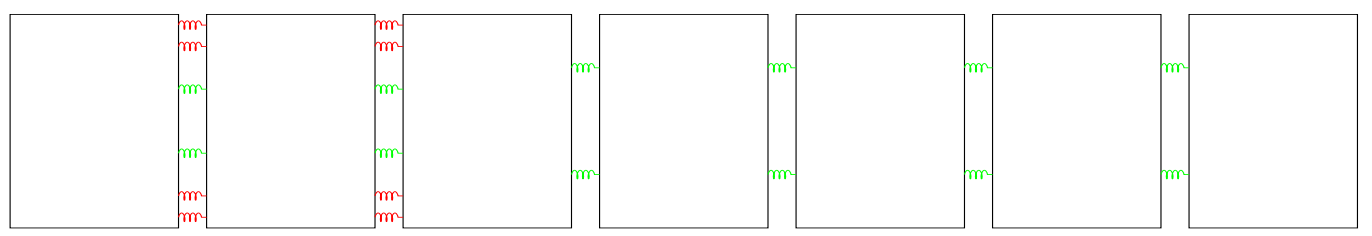

Figure 6.17: Representation of a cantilever beam using adaptive AEM springs

\subsubsection{Transition Point Locator}

There are two different approaches that were used to locate the transition point in a cross-section. The calculation of the transition point is based on determining between which two springs there was a difference in the elasticity of the spring. An initial guess of 10 springs is recommended so there are at least two springs in each region between where the transition point will occur. This is to ensure that there are two points to create a line, and an intersection point between these two lines is calculated. Consider the intersection of two lines $L_{1}$ defined by the points $\left(x_{1}, y_{1}\right)$ and $\left(x_{2}, y_{2}\right)$, and line $L_{2}$ defined by points $\left(x_{3}, y_{3}\right)$ and $\left(x_{4}, y_{4}\right)$, the intersection of the point $P_{x}, P_{y}$ is defined using determinants, as displayed in Equation 6.75:

$$
\begin{gathered}
P_{x}=\frac{\left(x_{1} y_{2}-y_{1} x_{2}\right)\left(x_{3}-x_{4}\right)-\left(x_{1}-x_{2}\right)\left(x_{3} y_{4}-y_{3} x_{4}\right)}{\left(x_{1}-x_{2}\right)\left(y_{3}-y_{4}\right)-\left(y_{1}-y_{2}\right)\left(x_{3}-x_{4}\right)} \\
P_{y}=\frac{\left(x_{1} y_{2}-y_{1} x_{2}\right)\left(y_{3}-y_{4}\right)-\left(y_{1}-y_{2}\right)\left(x_{3} y_{4}-y_{3} x_{4}\right)}{\left(x_{1}-x_{2}\right)\left(y_{3}-y_{4}\right)-\left(y_{1}-y_{2}\right)\left(x_{3}-x_{4}\right)}
\end{gathered}
$$
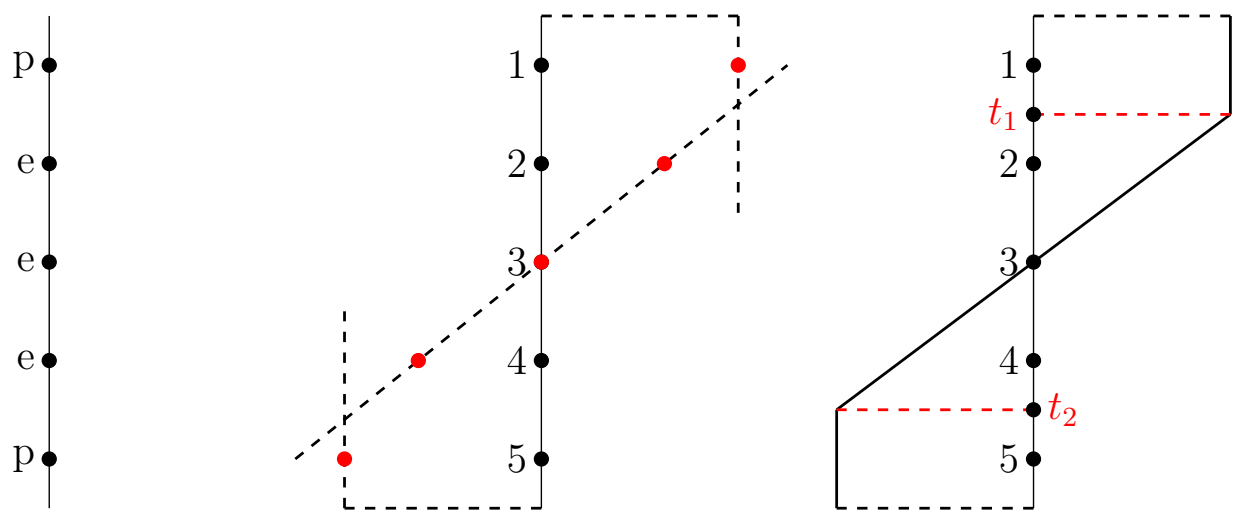

Figure 6.18: Transition Point locator

Figure 6.18 displays the process at which the transition point is located. Consider a cross-section between a pair of elements. The five points on the figure represent five springs between the cross-section. The first part of the figure shows 
"p" which demonstrates plastic, and "e" represents elastic. This explains that the spring is plastic or elastic. The way that the transition point is located is based on the fact that the transition will occur between two points that have different plasticity. So between spring 1 and 2 there should be a transition point, and another transition between spring 4 and 5. The second part of the figure shows the red points, which is the stress of each spring. Connecting the location of the stresses to one another shows where the intersection between the two lines will occur, and so the location of the transition point. Since the coordinates of the stress at each spring is known, using equation 6.75 the transition springs can be calculated. From knowing the transition points now the section can be separated where the springs are Gaussian distributed within each region.

\subsubsection{Examples}

In this section, different structural beams subject to incremental loading will be analysed using the adaptive Gaussian springs technique. First, consider a cantilever beam, fixed at one end and load applied at the free end. As mentioned every pair of elements starts with an initial 10 springs, and after the plasticity is determined for that load step, the springs are redistributed. If the element is totally elastic, then only 2 springs are required, while if it is elasto-plastic, a total of 6 springs are required. The difference in the MATLAB code for the conventional springs and the adaptive springs is adding an extra requirement in the second iteration by checking the elasticity and redistributing the springs. For elastoplastic cases usually five Newton Raphson iterations were required. Therefore, for an elasto-plastic case, the first iteration would have 10 springs, in the second iteration there is an evaluation of the springs and they are redistributed with 6 total springs, and finally from the third iteration on, the analysis continues for just 6 springs. This is presented in examples as follows.

\section{Cantilever Beam}

Considering a cantilever beam, an elastic analysis and an elasto-plastic analysis is performed. The cantilever beam with its deflected shape is shown in Figure 6.19. From the stress diagram, the first three spring sets were elasto-plastic, while the 
remainder of the spring sets are totally elastic. The transition point location for the three spring sets is shown in Figure 6.22. The new springs distribution in every spring set is shown in Figure 6.23.

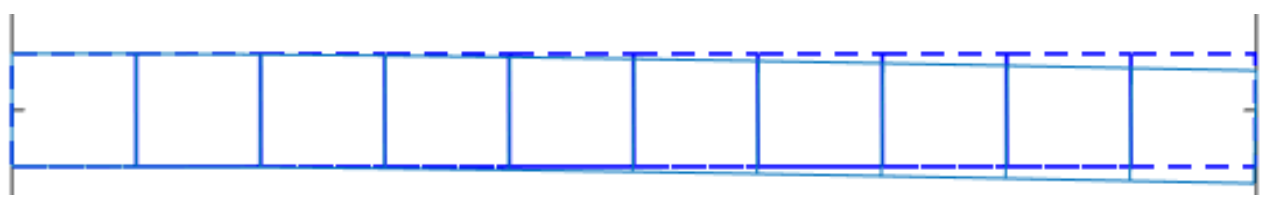

Figure 6.19: Cantilever beam with 10 elements
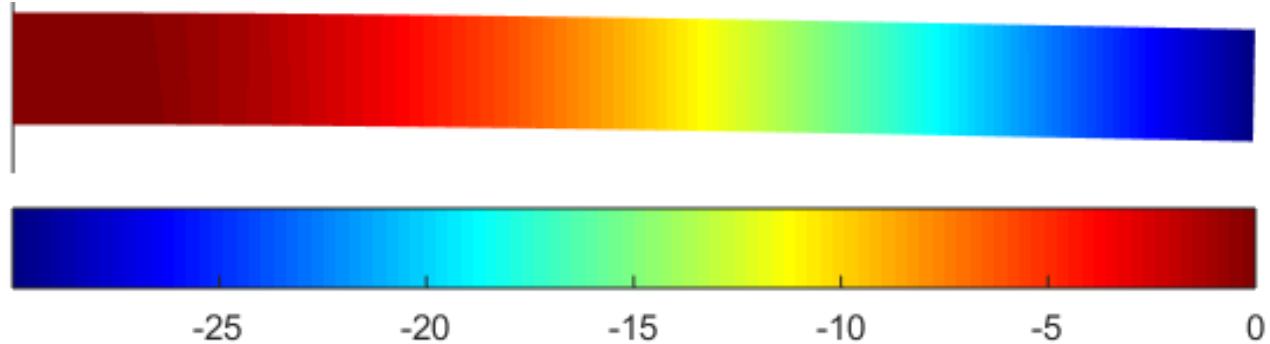

Figure 6.20: Deflection of cantilever beam with adaptive springs

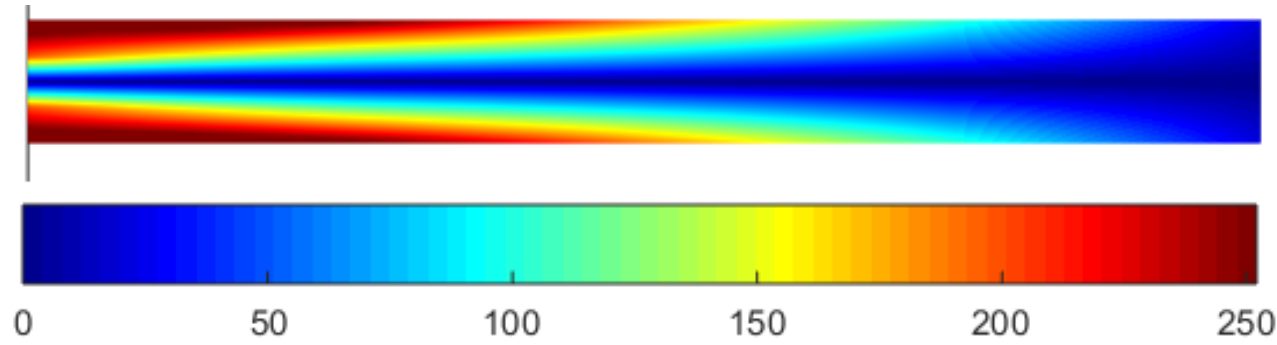

Figure 6.21: Normal stress of cantilever beam with adaptive springs

Figure 6.24 displays the stress distribution at every cross-section and the points are the spring locations. Figure 6.25 is the convergence rate of the Newton Raphson iteration for the beam with transition points. Also, it is worth mentioning that the transition points calculation did not change the convergence rate from the conventional springs, so with less springs accurate results were obtained. In the scheme of larger scale structures, the number of springs can be significantly reduced. 


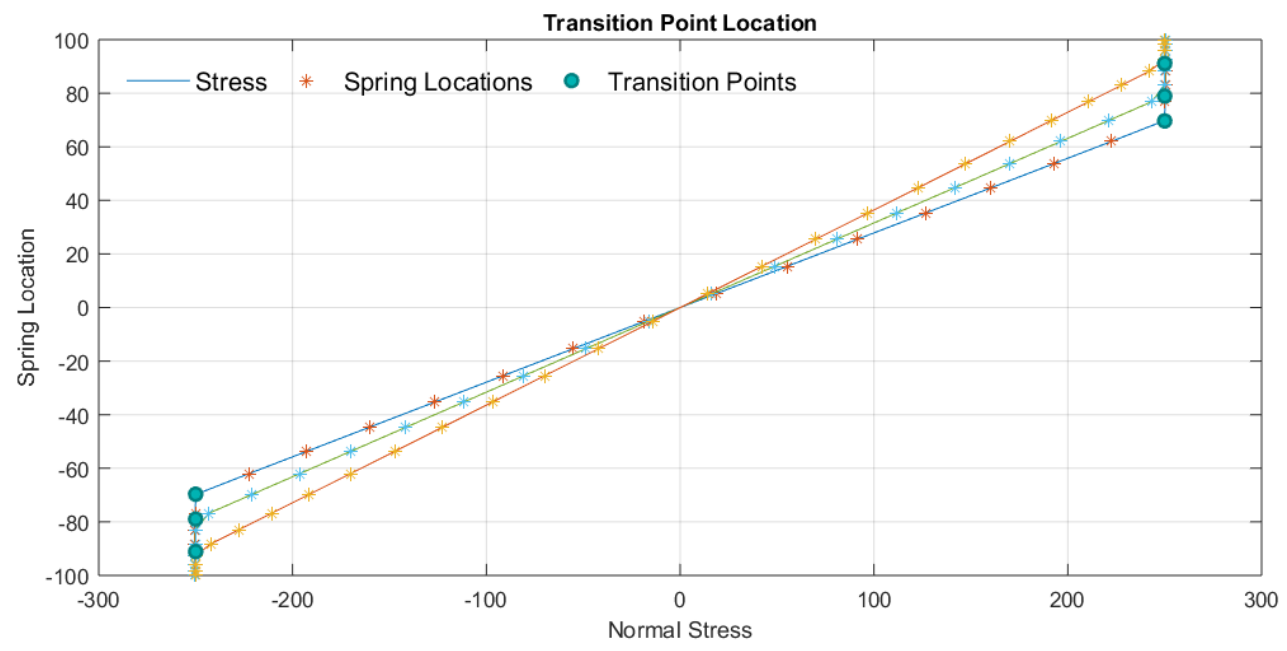

Figure 6.22: Spring sets that are elasto-plastic

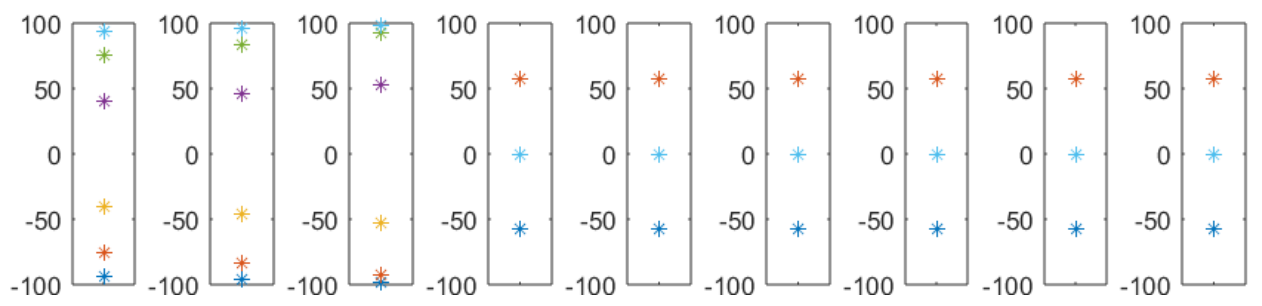

Figure 6.23: Springs distribution between elements
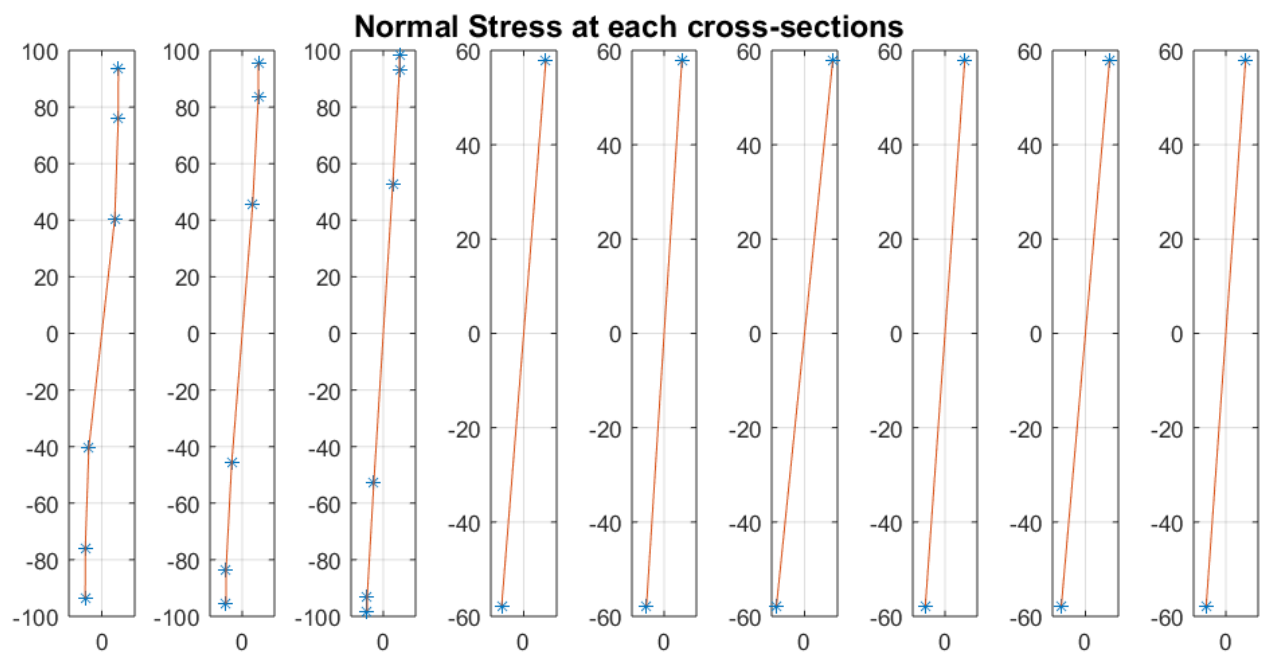

Figure 6.24: Normal stress distribution at every cross-section

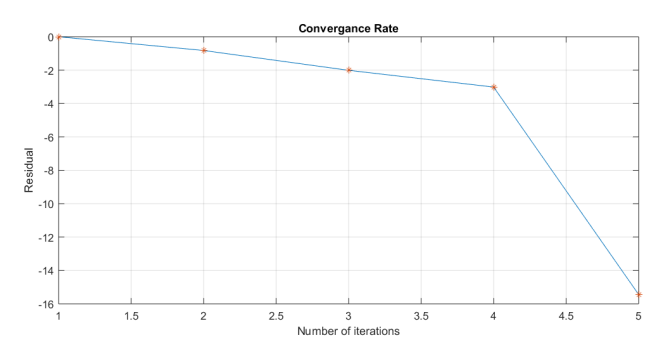

Figure 6.25: Converge Rate of Newton Raphson
Table 6.2: Residual at each iteration

\begin{tabular}{c|c}
\hline Iteration & Residual \\
\hline 1 & 1 \\
2 & $1.50 \times 10^{-1}$ \\
3 & $9.75 \times 10^{-3}$ \\
4 & $9.67 \times 10^{-4}$ \\
5 & $3.64 \times 10^{-16}$
\end{tabular}




\section{Fixed End Beam}

Figure 6.28 shows the stress distribution at the elasto-plastic cross-sections. The deflection of the beam is shown in Figure 6.26. Figure 6.27 is the normal stress distribution for the fixed end beam. As can be seen, the maximum stresses occur at the ends of the beam and at the location of the applied load at the midspan. Therefore it is expected that elasto-plasticity occurs at the ends and midspan. This is correctly conveyed in Figure 6.29. The stress distribution with the springs displayed as points along the beam cross-sections are shown in Figure 6.30.

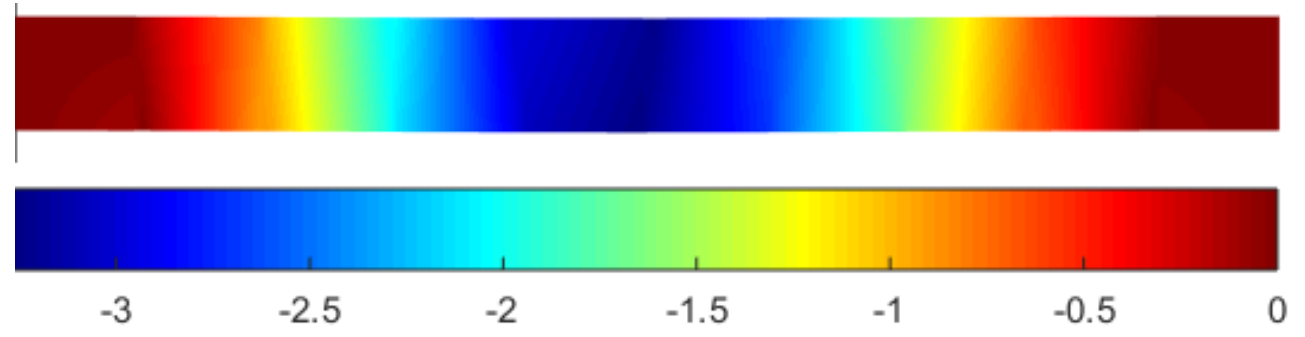

Figure 6.26: Deflection of FEB with adaptive springs

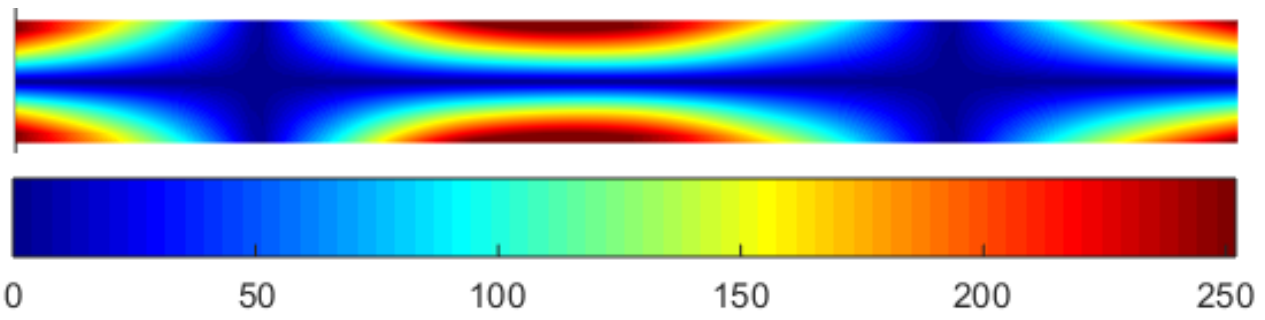

Figure 6.27: Normal stress distribution for Fixed End Beam

The results for the adaptive springs showed good accordance with the original AEM and in comparison to the Gaussian AEM. The convergence rate did not change. The main advantage of the method is that the number of springs is significantly reduced. For perfectly elastic cases, only 2 springs per element are needed, while for elasto-plastic cases 6 springs are needed. 


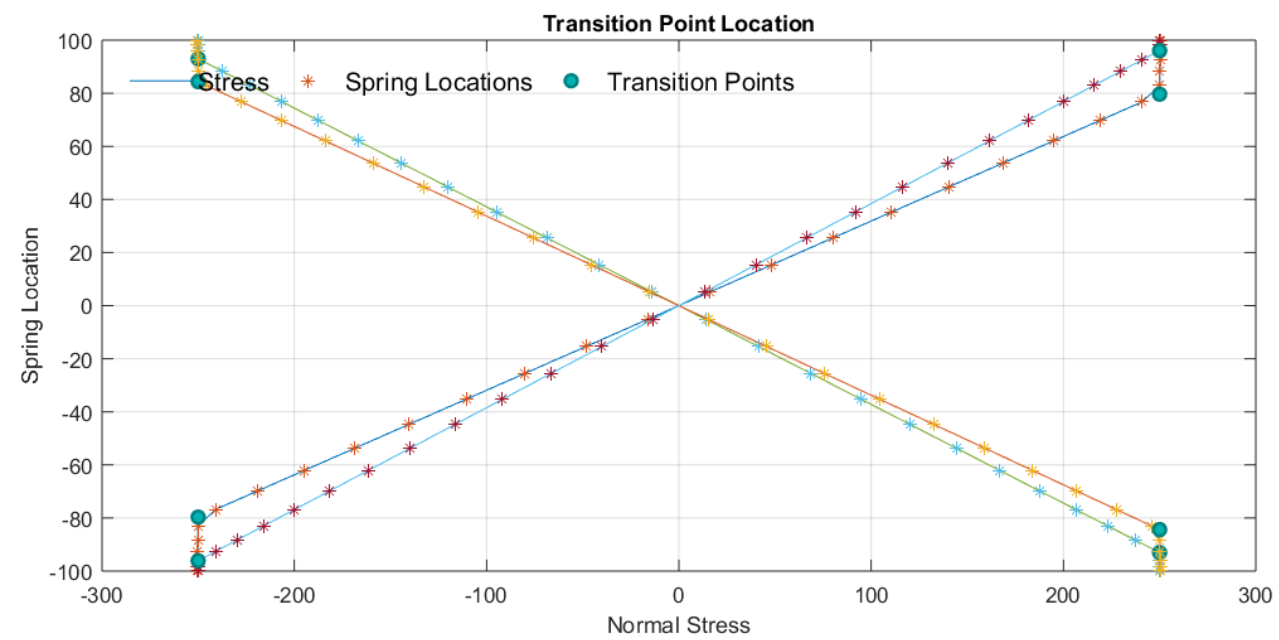

Figure 6.28: Stress with adaptive springs for FEB
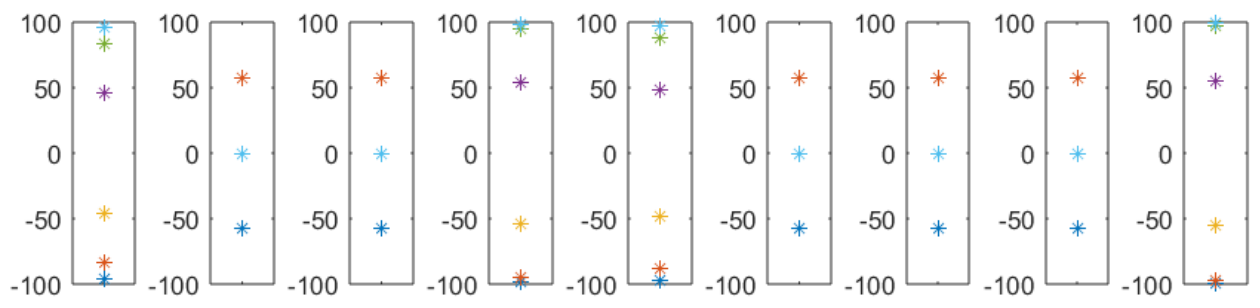

Figure 6.29: New springs distribution for FEB
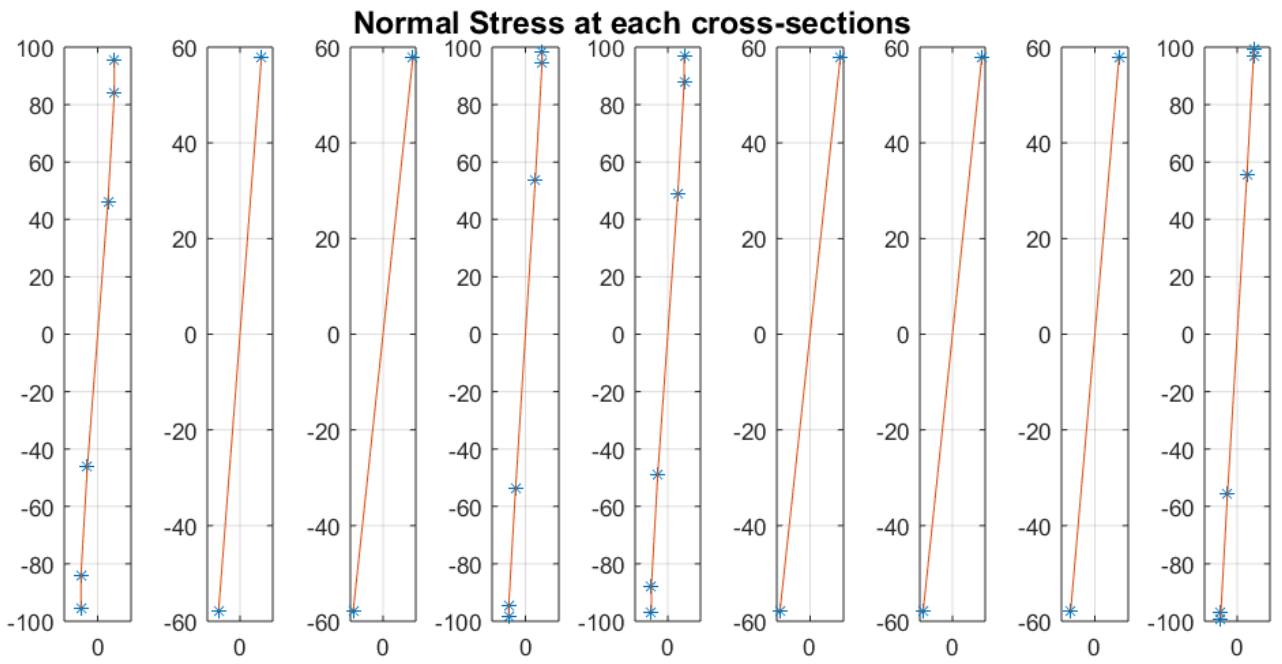

Figure 6.30: New stress distribution for FEB

\subsection{Conclusion}

The Applied Element Method has been modified for elasto-plastic problems by efficiently distributing springs using the Gaussian quadrature. The Hardening plasticity algorithm was used for representing the elasto-plastic material. The 
Newton Raphson iteration scheme was implemented, and resulted in quadratic convergence. Moreover, an adaptive method was used to model the behaviour of the elements in elasto-plasticity, where a total of 6 springs is required for elastoplastic elements, and 2 springs for elastic elements. This revelation allowed an accurate analysis of structural members while have a remarkably low number of springs per element.

The material damage behaviour is presented in the next chapter. 


\title{
Part III
}

Formulation of Damage, Fracture and Progressive Collapse in

Gaussian Based AEM \&

\author{
Applications
}




\section{Chapter 7}

\section{Material Damage Model \& Dynamic Analysis}

In the previous chapters, the elastic and elasto-plastic material behaviour was successfully modelled for applications in AEM. The modelling of progressive collapse is an extension of these methods and is presented in this chapter. Modelling progressive collapse entails that the material must undergo damage and fracture first. One material behaviour that exhibits material damage is softening material behaviour. The following sections describe material softening and constitutive equations developed to model the collapse of structures.

\subsection{Softening Material Behaviour}

Strain softening is the deterioration of material strength with increasing strain [74]. This is usually found in damaged quasi brittle materials such as fibre reinforced composites and concrete [74]. Other materials include rocks, some soils, wood, sea ice, fibre-reinforced concretes, asphalt concretes, polymer concretes, ceramics and some metals [75]. Strain softening has been considered as inadmissible in continuum mechanics [75]- [79]. Hadamard [80] pointed out that the wave velocity becomes imaginary and the differential equation becomes elliptic if the tangent moduli matrix is not positive definite [75].

A major part of construction materials around the world include materials from the above listed. For this reason, there is an importance to modelling the 


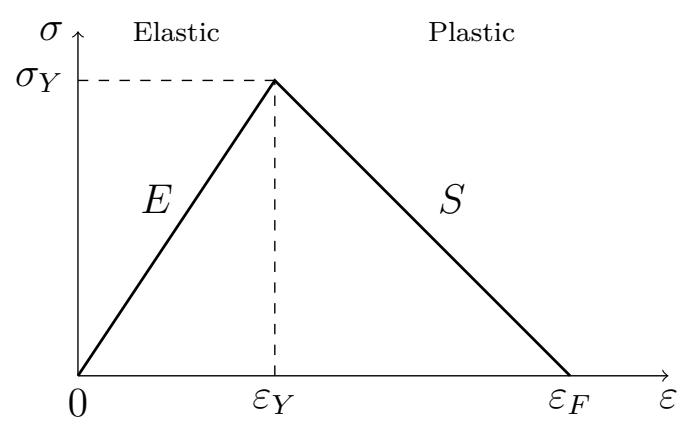

Figure 7.1: Strain Softening Material Behaviour

softening materials behaviour after materials exceed their elastic limit. In this section the return mapping algorithm for softening is developed. The strain softening behaviour is exhibited when failure occurs by progressive damage [75].

\section{Return Mapping Algorithm for Softening Model}

The stress-strain diagram of the softening materials is presented as a bilinear model, with the slope as a positive $E$ in the elastic region, and a declining negative slope $S$ that represents the strain-softening behaviour. Based on the constitutive model derived for hardening, similar principles are implemented to develop the softening behaviour. Figure 7.1 is a representation of the material model presented as a stress-strain diagram. In AEM each spring will have a unique stress-strain diagram. Once the strain exceeds the elastic yield limit $\varepsilon_{Y}$, it enters the plastic region and the slope is the negative $S$. Then when the strain exceeds the failed strain $\varepsilon_{F}$ the material is considered to have been totally damaged in that region. Since each spring represents a tributary area of a section of an element, then a failed spring physically represents that that portion of the region has failed.

The return mapping algorithm is described as follows. First the strain is calculated from the beam deflection, and the strain increment is obtained. The trial stress is calculated based on using $E$, shown in Equation 7.1. Note that there are two different strain definitions in the equation: $\varepsilon$ is the strain and $\epsilon$ is the plastic softening strain calculated in Equation 7.6. $\epsilon$ keeps track of the plastic strain. If the stress and strain do not exceed the yield limit state yet, then $\epsilon^{p}$ is zero because there is no plastic strain yet. So Equation 7.1 will be simply 
$\sigma_{n+1}^{\text {trial }}=E\left(\varepsilon_{n+1}\right)$

$$
\sigma_{n+1}^{\text {trial }}=E\left(\varepsilon_{n+1}-\epsilon_{n}^{p}\right)
$$

Similar to the 1D hardening plasticity, a yield criterion is developed for the softening material behaviour. The yield condition is used to determine when yield occurs, and is denoted by $f$. The yield function results in either a positive or negative value. The yield function $f$ is shown in Equation 7.2.

$$
f_{n+1}^{\text {trial }}=\left|\sigma_{n+1}^{\text {trial }}\right|-\left(\sigma_{Y}^{0}+S\left(\varepsilon_{n+1}-\varepsilon_{Y}^{0}\right)\right)
$$

The yield function is examined to determine which region the spring is currently located: elastic, plastic or failed. If the strain is larger than the failed strain then the spring has failed. If the yield criteria is less than zero this means that the difference of the yield stress and the calculated softening stress is smaller than the current trial stress, so the location of the trial stress is before the yield stress meaning that it is still in the elastic region, and the plasticity parameter $\Delta \lambda=0$. In the elastic region linear stiffness of a spring is calculated from Equation 7.3.

$$
K_{n}=\frac{E A}{l} ; \quad K_{s}=\frac{G A}{l}
$$

Otherwise the spring is in the plastic region. The plasticity parameter $\Delta \lambda$ is calculated and is greater than zero, shown in Equation 7.4.

$$
\Delta \lambda=\frac{f_{n+1}^{\text {trial }}}{E}
$$

The new stress $\sigma_{n+1}$ is then calculated (corrector-step) Equation 7.5, and the corresponding plastic strain $\epsilon_{n+1}^{p}$.

$$
\begin{gathered}
\sigma_{n+1}=\sigma_{Y}^{0}+S\left(\varepsilon_{n+1}-\varepsilon_{Y}\right) \\
\epsilon_{n+1}^{p}=\epsilon_{n}^{p}+\Delta \lambda \operatorname{sign}\left[\sigma_{n+1}^{\text {trial }}\right]
\end{gathered}
$$


The nonlinear stiffness of the springs are calculated in Equation 7.7.

$$
\begin{aligned}
K_{n} & =\frac{C_{n} A}{l} ; C_{n}=\frac{E H}{E+H} \\
K_{s} & =\frac{C_{s} A}{l} ; C_{s}=\frac{G H}{G+H}
\end{aligned}
$$

For a failed spring, the stiffness is set as zero.

The return mapping softening algorithm is presented in Box 7.1.

\section{Box. 7.1.1: Return Mapping Softening algorithm}

1. Loop for every element

(a) Loop for every spring

(b) Obtain new strain $\epsilon_{n+1}=\epsilon_{n}+\delta \epsilon_{n+1}$

(c) Evaluate elastic predictor

$$
\begin{gathered}
\sigma_{n+1}^{\text {trial }}=E\left(\varepsilon_{n+1}-\epsilon_{n+1}^{p}\right) \\
f_{n+1}^{\text {trial }}=\left|\sigma_{n+1}^{\text {trial }}\right|-\left(\sigma_{Y}+S\left(\varepsilon_{n}-\varepsilon_{Y}\right)\right)
\end{gathered}
$$

(d) Check yield criterion

$$
\text { if } \begin{cases}\varepsilon_{n}>\varepsilon_{f} & \text { spring failed } \\ f_{n+1}^{\text {trial }} \leq 0 & \Delta \lambda=0 \\ f_{n+1}^{\text {trial }}>0 & \Delta \lambda>0\end{cases}
$$

(e) If spring failed set stiffness of spring to zero.

(f) else Elastic step (if $f_{n+1}^{\text {trial }} \leq 0$ ). Calculate linear stiffness of springs.

(g) Plastic step (if $f_{n+1}^{\text {trial }}>0$ )

$$
\begin{gathered}
\Delta \lambda=\frac{f_{n+1}^{\text {trial }}}{E} \\
\sigma_{n+1}=\sigma_{Y}^{0}+S\left(\varepsilon_{n+1}-\varepsilon_{Y}\right) \\
\epsilon_{n+1}^{p}=\epsilon_{n}^{p}+\Delta \lambda \operatorname{sign}\left[\sigma_{n+1}^{\text {trial }}\right]
\end{gathered}
$$

Calculate nonlinear stiffness of springs.

(h) Repeat from step for more springs within the same pair of elements

2. Assemble internal forces in global force vector, and stiffness in global stiffness matrix

3. Repeat for next pair of elements 


\section{Softening Return Mapping algorithm schematic representation}

The return mapping algorithm is presented schematically for better explanation of the formulation.

1. Trial stress predictor

First consider that the strain $\varepsilon_{n}$ has not exceeded the yield strain $\varepsilon_{y}$, but the next increment $\varepsilon_{n+1}$ exceeds $\varepsilon_{y}$, as shown in Figure 7.2. Since there is no plastic strain, the corresponding $\sigma_{n+1}^{\text {trial }}$ is calculated as $\sigma_{n+1}^{\text {trial }}=E \varepsilon_{n+1}$.

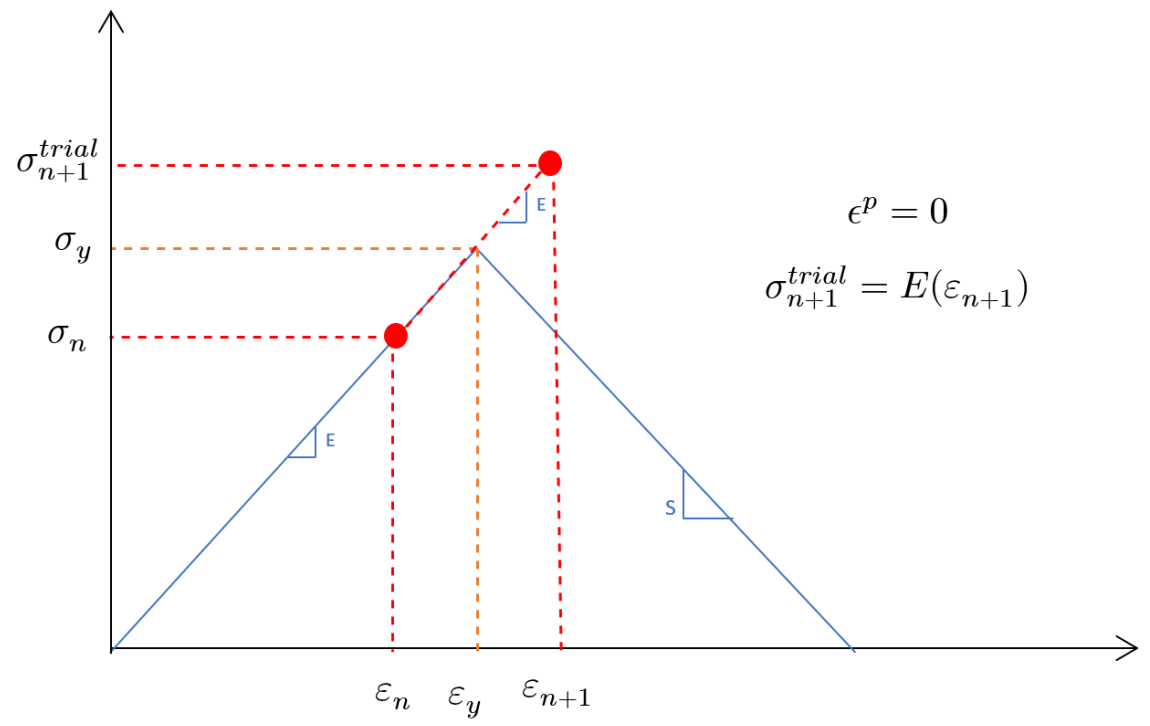

Figure 7.2: Trail stress predictor calculation

2. Calculate $f_{n+1}$

Now the yield function must be evaluated. Recall that $S$ is negative, so the purple line presents $\sigma_{Y}+S\left(\varepsilon_{n+1}-\varepsilon_{y}\right)$ and is less than $\sigma_{Y}$ because it is being subtracted. The yield function $f_{n+1}^{\text {trial }}$ calculation is displayed in Figure 7.3.

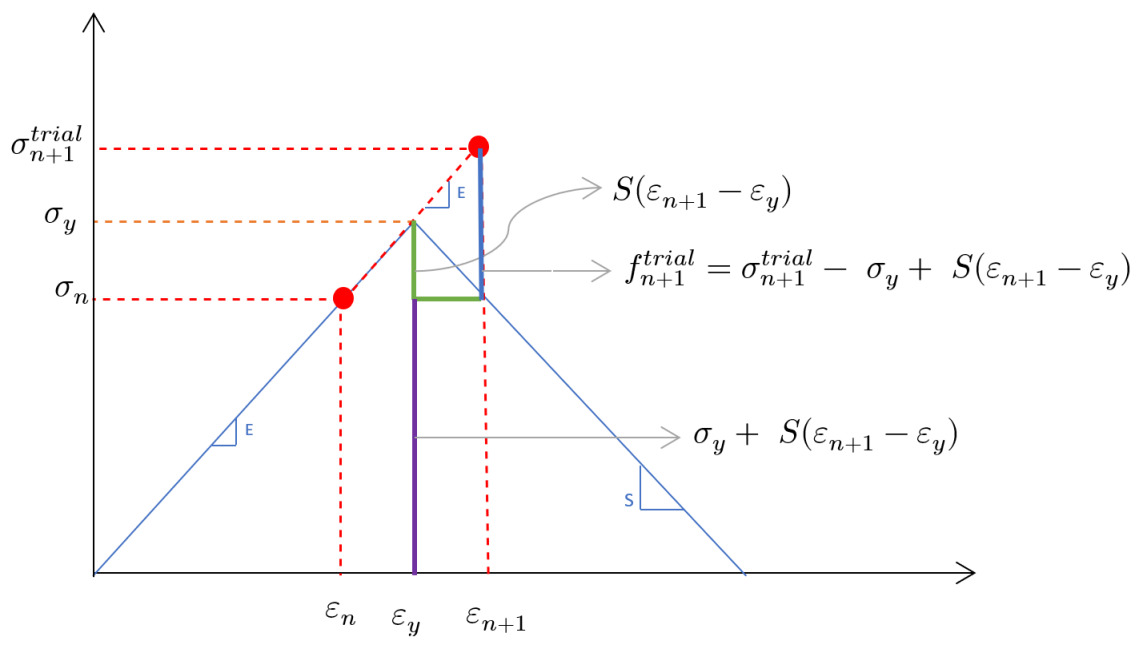

Figure 7.3: Demonstrating the trial function 
3. Calculate $\Delta \lambda$

Since $f_{n+1}^{\text {trial }}$ is now known, $\Delta \lambda$ is used to calculate the plastic strain $\epsilon^{p}$ just by using the elastic slope $E . \Delta \lambda$ is represented in Figure 7.4.

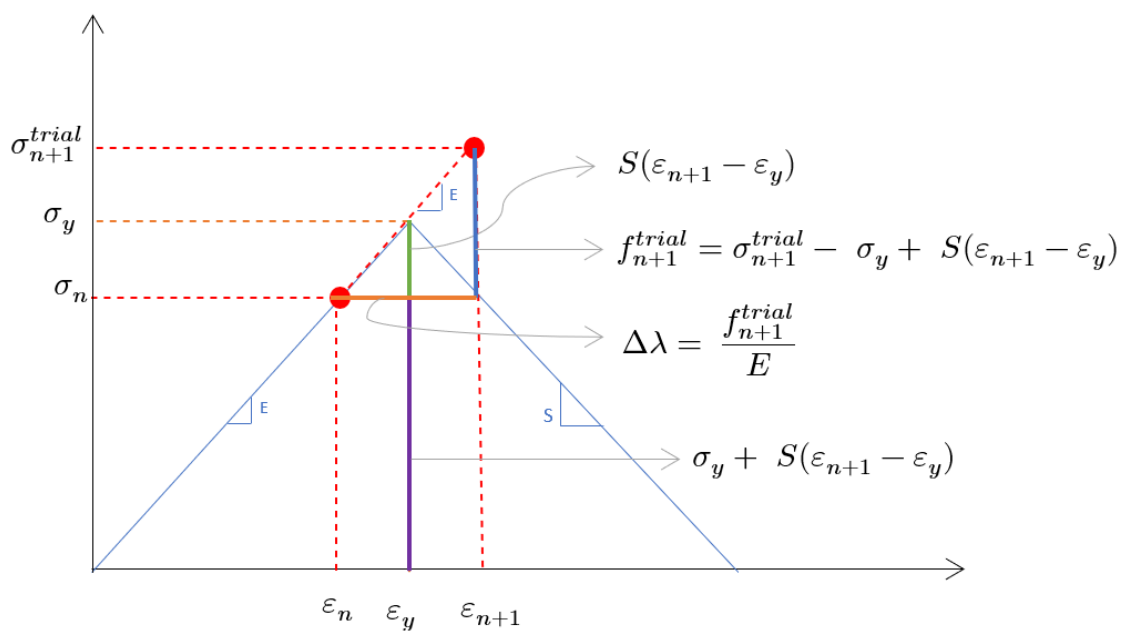

Figure 7.4: Representation of $\Delta \lambda$

4. Obtain the plastic strain $\epsilon^{p}$.

The plastic strain is shown in Figure 7.5. Since there is no previous plastic strain because this is the first time step in which the strain has exceeded the yield strain, the plastic strain at this time step $=\Delta \lambda$.

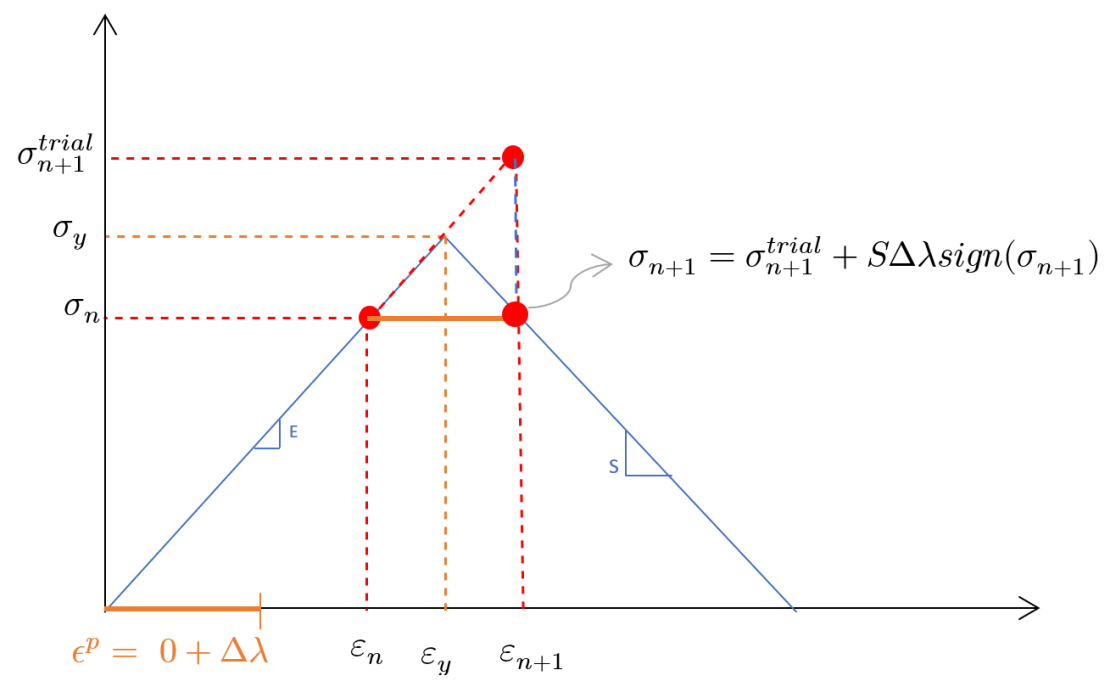

Figure 7.5: Plastic strain

5. Now that the strain and corrected stress is obtained the corresponding stiffness of the spring is obtained as well as the internal force.

6. Begin next time step and calculate the trial stress. Since there is a plastic strain, $\epsilon^{p}$ is included in the trial stress equation. $\varepsilon_{n+1}-\epsilon^{p}$ is schematically represented in Figure 7.6, along the x-axis. The trial stress is then obtained using the Young's Modulus E. 


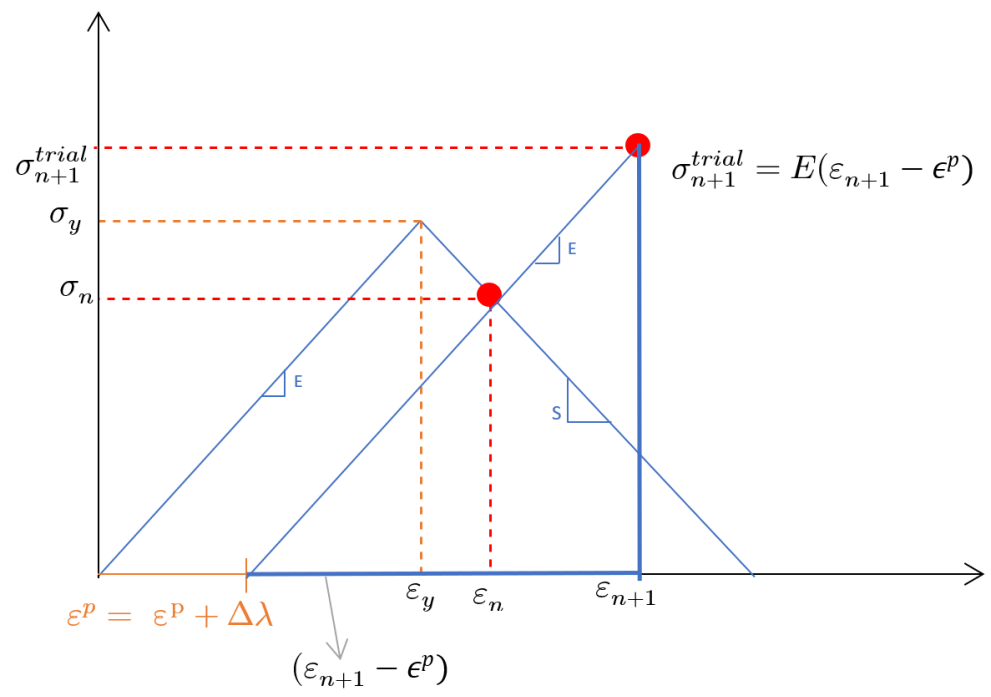

Figure 7.6: Trial stress calculation

7. Similar to the previous time step $f_{n+1}^{\text {trial }}$ is obtained, and shown in Figure 7.7 .

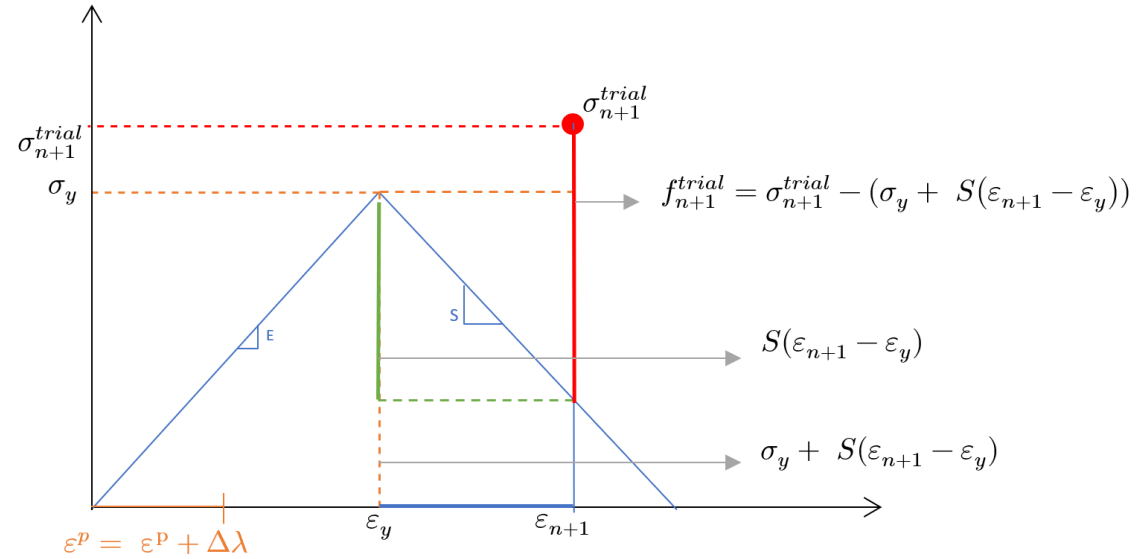

Figure 7.7: $f_{n+1}^{\text {trial }}$ calculation

8. $\Delta \lambda$ and new $\epsilon^{p}$ can now be obtained, and is shown in Figure 7.8.

\subsubsection{Return-Mapping Algorithm verification}

In this section the verification of the Predictor-Corrector algorithm is presented and the Newton Raphson convergence is examined. Figures 7.9 and 7.10 show the return mapping for the predictor and corrector at different time steps. The green points describe the predictors, and the red points describe the correctors. From the first figures it is clear that when the spring is in the elastic stage it will follow the Young's Modulus E, and therefore the predictor takes the next stress 


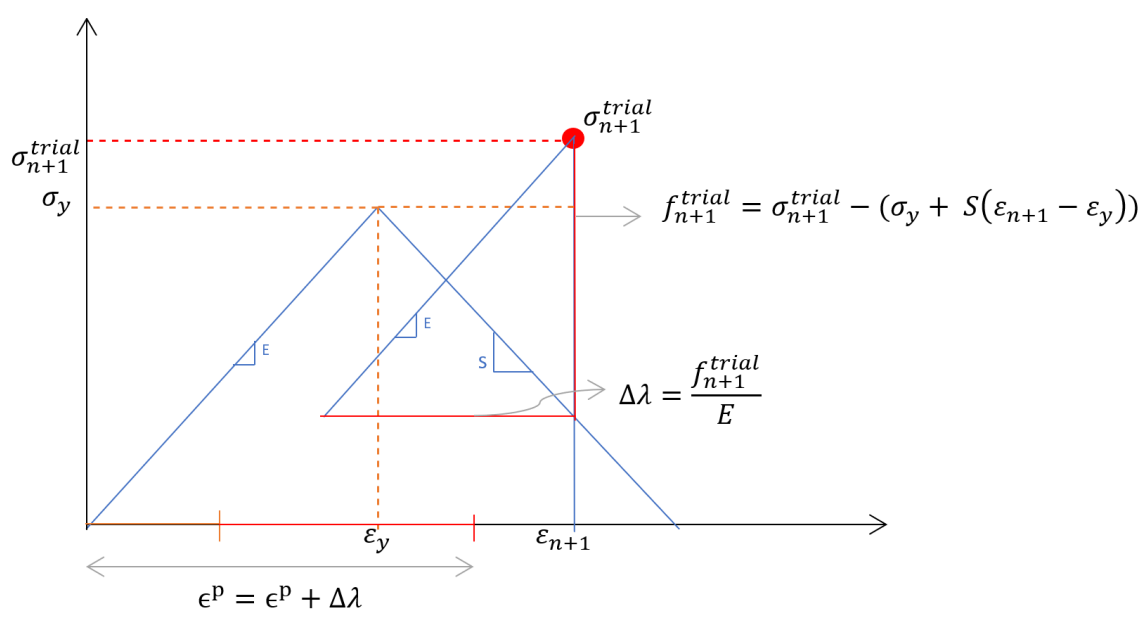

Figure 7.8: $\Delta \lambda$ and $\epsilon^{p}$ calculation

time step to the correct location, and no correction is actually needed. Once the stress exceeds the elastic limit, the predictor predicts the stress along the elastic modulus, and later the algorithm corrects the stress to the correct location along the Softening modulus. Later the unloading stage is shown. Since the unloading occurs after the yield stage it follows a new path which is parallel to the modulus $E$, however, since there is permanent plastic strain, it will not go back to zero. Finally in the reloading stage, the predictor predicts along the new slope of $E$, and once it intersects with the softening slope, it starts to correct to the softening slope rather than $E$. The point of intersection is considered the new yield stress.

\subsubsection{Failed Elements}

Newton Raphson convergence cannot be obtained with softening damage materials due to the singularity of the stiffness matrix. The stress-strain diagram of the predictor corrector method shows a good behaviour of the strain softening, however there is no Newton Raphson convergence. Also the eigen values for the system are negative after the yield point is surpassed. For this reason a dynamic model must be used. After failure or crack occurs in the static analysis (element separation), the stiffness matrix becomes singular, causing inaccurate solutions since it cannot be invertible. Solving the problem in a dynamic case will eliminate this issue. 


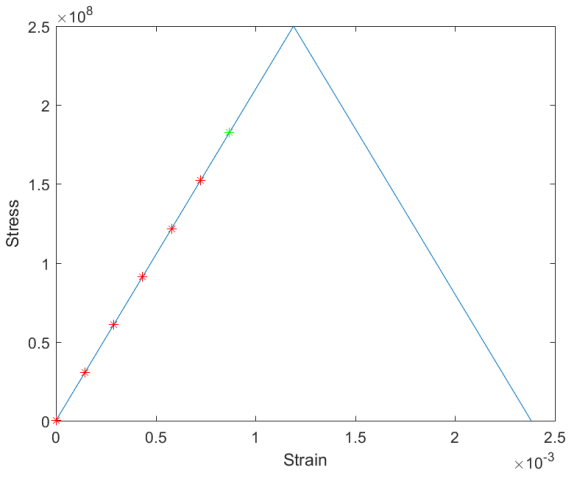

(a) predictor

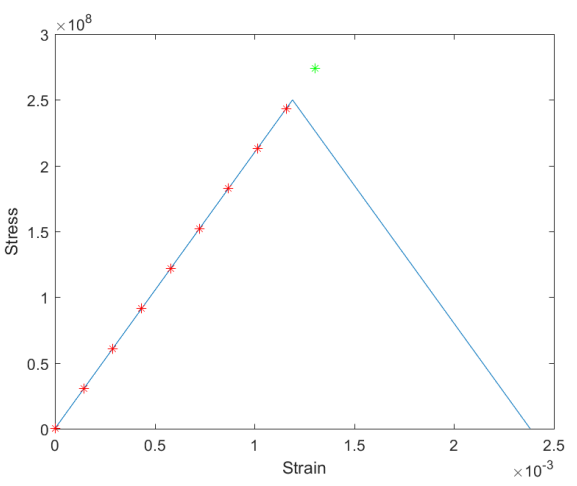

(c) predictor

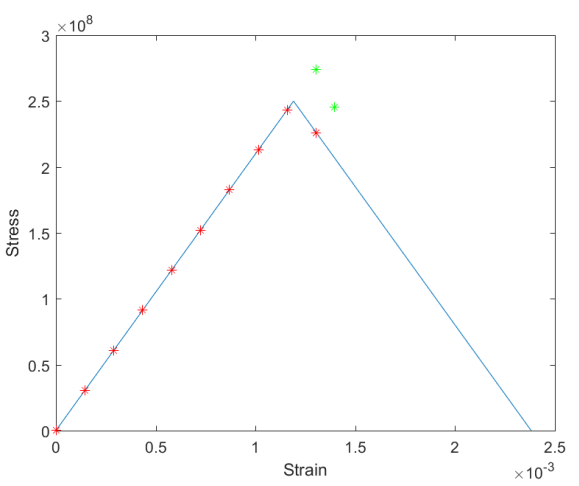

(e) predictor

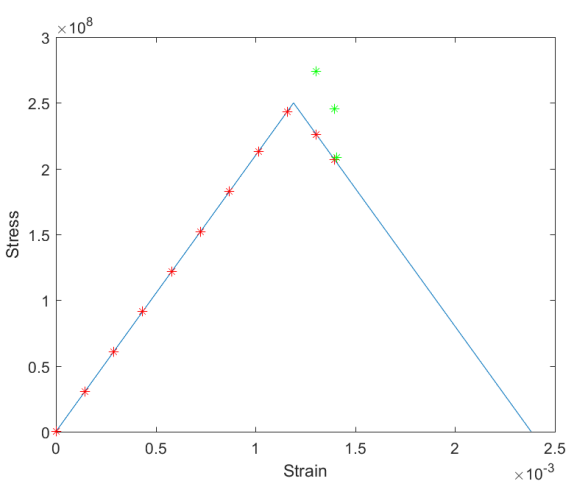

(g) predictor

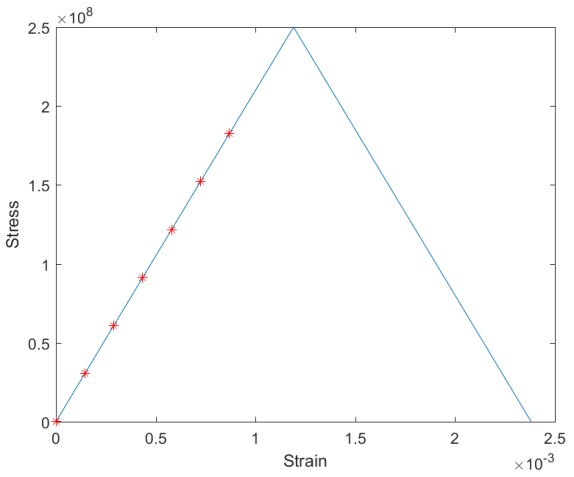

(b) corrector

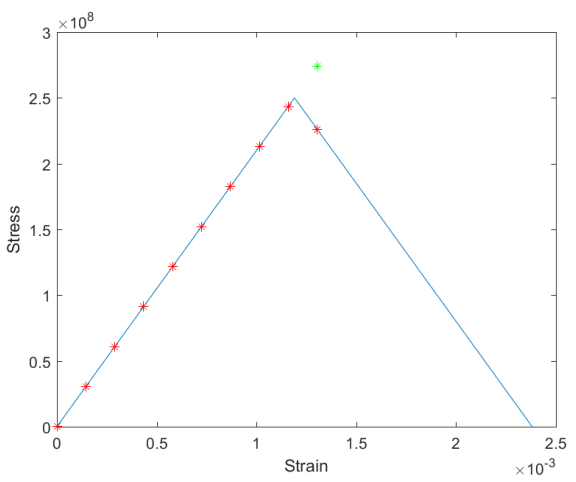

(d) corrector

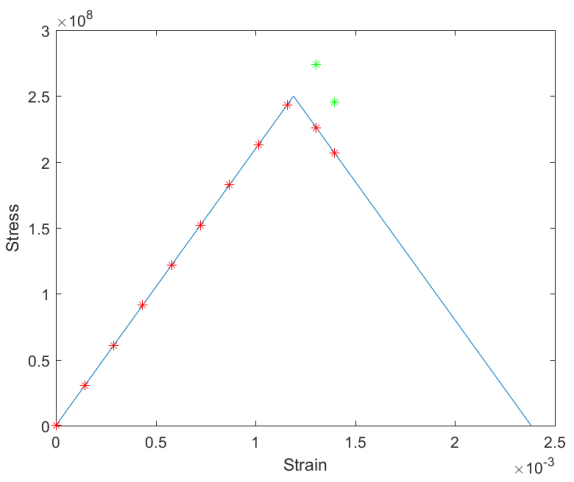

(f) corrector

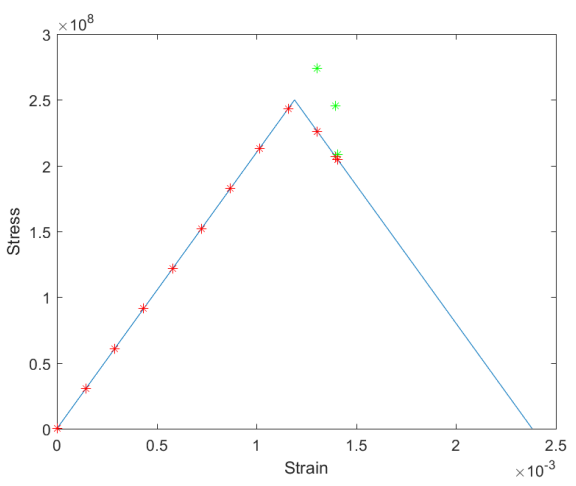

(h) corrector

Figure 7.9: Predictor-corrector Softening model 


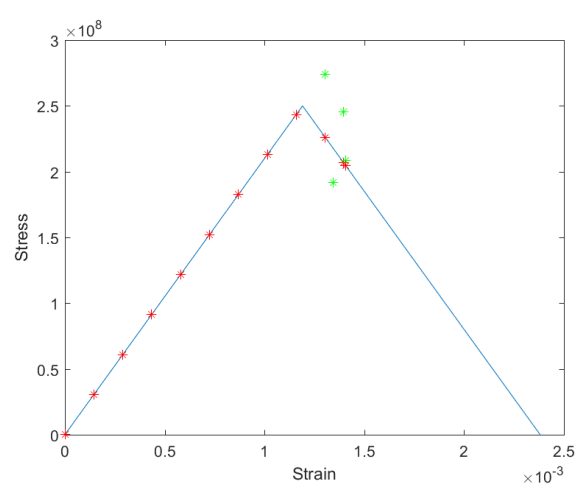

(a) predictor

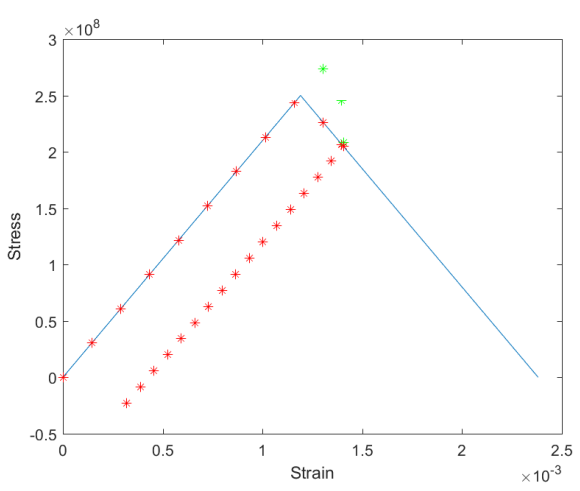

(c) predictor

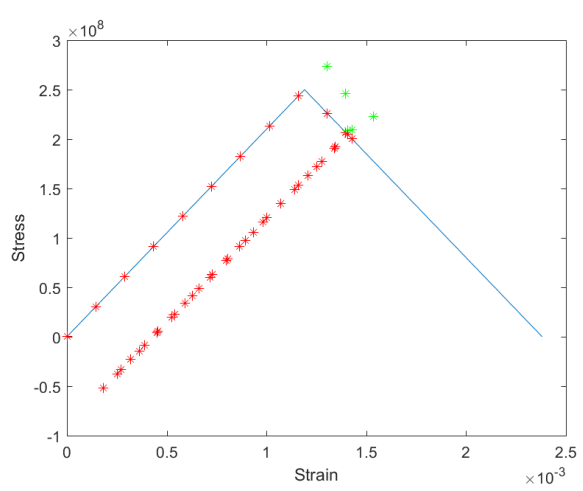

(e) predictor

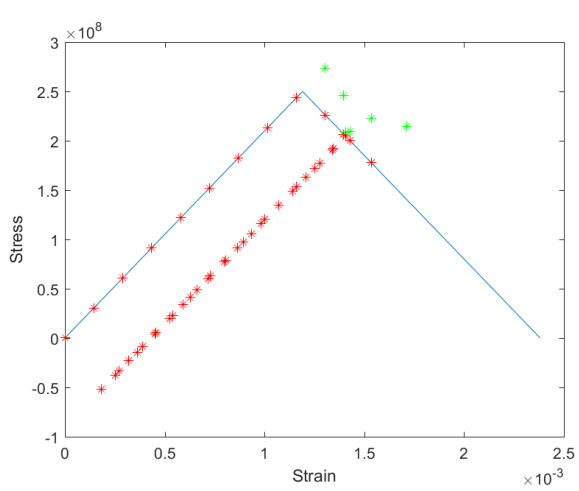

(g) predictor

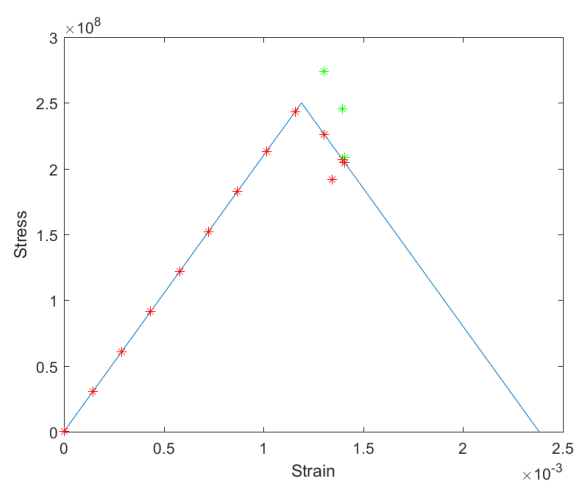

(b) corrector

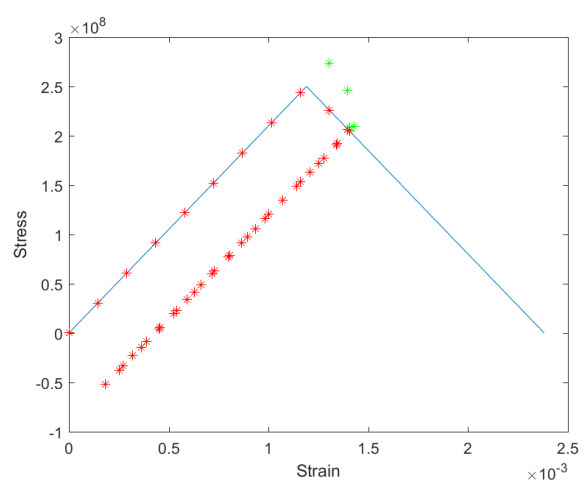

(d) corrector

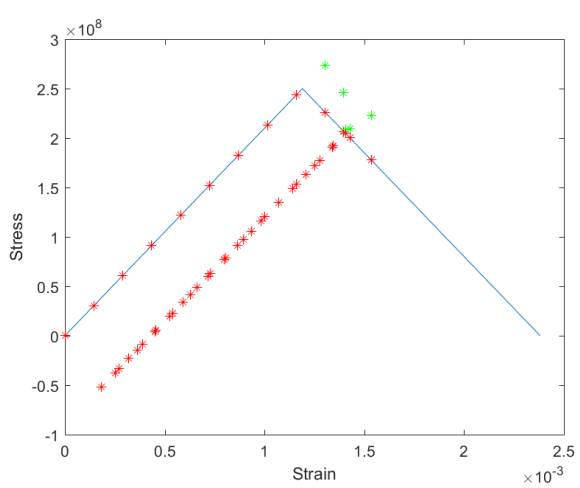

(f) corrector

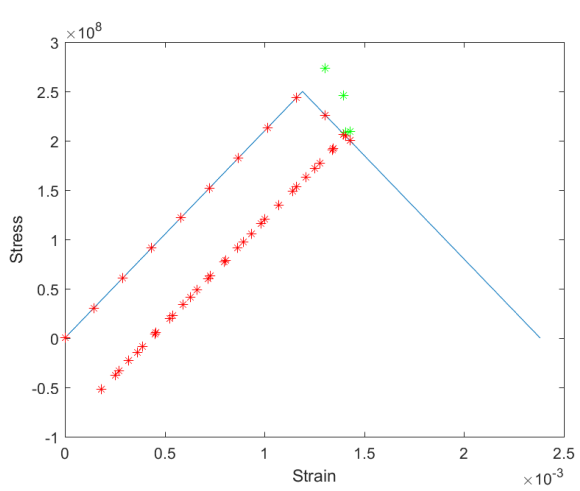

(h) corrector

Figure 7.10: Predictor-corrector Softening model 


\subsection{Introduction to Dynamic Analysis}

In this section, the formulation of the dynamic aspect in AEM is presented. A general formulation of the equation of motion for multiple degrees-of-freedom systems subject to external forces or earthquake ground motion is shown. Based on the presented formulations, the most applicable numerical time integrator for use in progressive collapse in AEM is selected. The equations for multiple degrees-of-freedom system (MDOF) are presented, since the scope of this thesis covers MDOF systems.

\subsubsection{Equation of Motion for MDOF Systems}

Consider the two story shear building shown in Figure 7.11a, with lumped mass at each floor level. The system has two degrees-of-freedom; lateral displacements $u_{1}$ and $u_{2}$ in the direction of the x-axis. The forces acting on the frame are in Figure $7.11 \mathrm{~b}$, where $f_{S}$ is the resisting force, $f_{D}$ is the damping force and $p(t)$ is the external applied force. The derivation of these forces is shown in the following section.

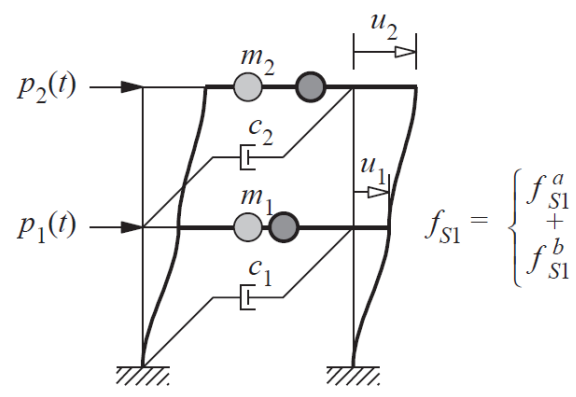

(a)

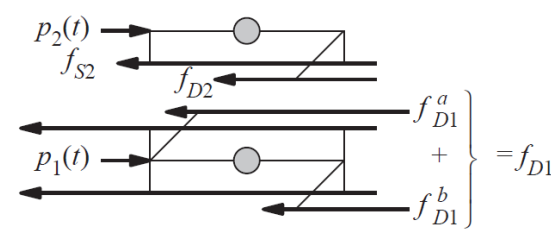

(b)

Figure 7.11: (a) Two story shear frame (b) Forces acting on frame [81]

\subsubsection{Equations of Motion for Linear Systems}

In a single degrees-of-freedom linear system the relationship between the lateral force and the deformation $u$ is linear and shown in Equation 7.8.

$$
f_{s}=k u
$$


where $k$ is the lateral stiffness of the system, $f_{s}$ is the external force, and the internal force is equal and opposite to $f_{s}$ [81]. The linear relationship implies that the loading and unloading curves are identical. For structures undergoing cyclic deformations, from earthquakes for instance, the initial loading curve is nonlinear at larger amplitudes of deformation, and the unloading and loading curves may differ from each other. This system is inelastic [81]. The resisting force is an implicit function of deformation

$$
f_{s}=f_{s}(u)
$$

Damping in structures is idealised since it is impossible to identify or mathematically represent the energy dissipation mechanisms in actual structures [81]. For simple degrees-of-freedom structures the damping is idealised by a linear viscous damper. The damping force is related to the velocity $\dot{u}$ across the linear viscous damper by

$$
f_{D}=c \dot{u}
$$

where $\mathrm{c}$ is the viscous damping coefficient and $\dot{u}$ is the velocity. Vibration experiments on actual structures provide the data for the damping coefficient. Therefore, the forces acting on a mass at an instance of time are the external force $p(t)$, the resisting force $f_{s}$ and the damping resistance force $f_{D}$. The resultant force along the $\mathrm{x}$-axis is $p-f_{S}-f_{D}$. The Newton's second law of motion gives

$$
p-f_{S}-f_{D}=m \ddot{u}
$$

which becomes

$$
m \ddot{u}+c \dot{u}+k u=p(t)
$$

Equation 7.12 can be extended for inelastic systems as

$$
m \ddot{u}+c \dot{u}+f_{S}(u)=p(t)
$$


The Newton's second law of motion for each mass is

$$
p_{j}-f_{S j}-f_{D j}=m_{j} \ddot{u}_{j}
$$

for $j=1$ and 2, Equation 7.14 can be written in matrix form

$$
\left[\begin{array}{cc}
m_{1} & 0 \\
0 & m_{2}
\end{array}\right]\left\{\begin{array}{l}
\ddot{u}_{1} \\
\ddot{u}_{2}
\end{array}\right\}+\left\{\begin{array}{l}
f_{D 1} \\
f_{D 2}
\end{array}\right\}+\left\{\begin{array}{l}
f_{S 1} \\
f_{S 2}
\end{array}\right\}=\left\{\begin{array}{l}
p_{1}(t) \\
p_{2}(t)
\end{array}\right\}
$$

which can be written as

$$
M \ddot{u}+f_{D}+f_{S}=p(t)
$$

where $M$ is the mass matrix. Since the elastic resisting force $f_{S}$ is related to the displacement vector and stiffness matrix $K$, the force can be represented as Equation 7.17. Also, the damping resistance force and the velocity vector are related the damping matrix $C$, as shown in Equation 7.18.

$$
\begin{aligned}
& f_{S}=K u \\
& f_{D}=C \dot{u}
\end{aligned}
$$

The equation of motion can now be written as

$$
M \ddot{u}+C \dot{u}+K u=p(t)
$$

For a general description of linear systems, with more than just two stories, the same approach is used. The degrees-of-freedom in the system will increase. The form of the equations of motion will remain the same as shown in Equation 7.19.

\subsubsection{Natural Vibration Frequency and Modes Calcula- tion}

The solution to the eigenvalue problem presented in this section gives the natural frequencies and the modes of a structural system [81]. The free vibration of an undamped system of one of the vibration modes can be represented using 
Equation 7.20.

$$
u(t)=q_{n}(t) \phi_{n}
$$

where the deflected shape $\phi_{n}$ does not vary with time. The time variation of the displacements is described by the simple harmonic function

$$
q_{n}(t)=A_{n} \cos \left(\omega_{n} t\right)+B_{n} \sin \left(\omega_{n} t\right)
$$

where $A_{n}$ and $B_{n}$ are constants determined from the initial conditions. Combining Equations 7.20 and 7.21 gives

$$
u(t)=\phi_{n}\left(A_{n} \cos \left(\omega_{n} t\right)+B_{n} \sin \left(\omega_{n} t\right)\right)
$$

Substituting in the undamped form Equation 7.19, $\ddot{u}$ is required.

$$
\begin{aligned}
& \dot{u}(t)=\phi_{n} \omega_{n}\left(-A_{n} \sin \left(\omega_{n} t\right)+B_{n} \cos \left(\omega_{n} t\right)\right) \\
& \ddot{u}(t)=\phi_{n} \omega_{n}^{2}\left(-A_{n} \cos \left(\omega_{n} t\right)-B_{n} \cos \left(\omega_{n} t\right)\right)
\end{aligned}
$$

So

$$
\ddot{u}(t)=-\phi_{n} \omega_{n}^{2} q_{n}(t)
$$

therefore, the undamped equation of motion can be rewritten as

$$
-M\left(\phi_{n} \omega_{n}^{2} q_{n}(t)\right)+K\left(q_{n}(t) \phi_{n}\right)=0
$$

Equation 7.26 can be satisfied either if $q_{n}(t)=0$, which is a trivial solution since it implies $u(t)=0$ and there is no motion in the system, or

$$
\left[K-\omega_{n}^{2} M\right] \phi_{n}=0
$$

The solution $\phi_{n}=0$ is also a trivial solution suggesting that there is no motion. Therefore, Equation 7.28 must be satisfied and is known as the characteristic 
equation.

$$
\operatorname{det}\left[K-\omega_{n}^{2} M\right]=0
$$

When Equation 7.28 is expanded, a polynomial of order $N$ in $\omega_{n}^{2}$ is retrieved. The $N$ roots, $\omega_{n}^{2}$, determine $N$ natural frequencies of vibration, and are known as the eigenvalues, characteristic values or normal values [81]. Once the natural frequency is known, Equation 7.27 can be solved to obtain the vector, $\phi_{n}$. For every $\omega_{n}$ there is a corresponding $\phi_{n}$. $\phi_{n}$ vectors are known as the eigenvectors, natural modes of vibration, or natural mode shapes of vibration [81].

\section{Natural vibration and modes for structural beams}

In MATLAB, there exists a function $[V, D]=E I G(A, B)$ that produces a diagonal matrix D of generalized eigenvalues and a full matrix $\mathrm{V}$ whose columns are the corresponding eigenvectors. In this case A would be the stiffness matrix, and B would be the mass matrix. Assuming a cantilever beam where the mass matrix $M$ and the stiffness matrix $K$ are already known. The eigenvalues and vectors would be easily retrieved using the function, where as previously noted, the eigenvalues correspond to the natural frequency $\omega_{n}$ and the eigenvectors are the natural modes of vibration $\phi_{n}$. The first six modal shapes of a cantilever beam using the function are shown in Figure 7.12, and for a fixed ends beam in Figure 7.13.

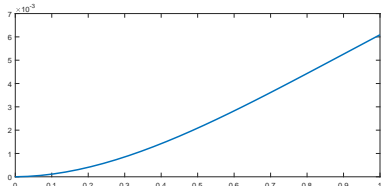

(a) Mode 1

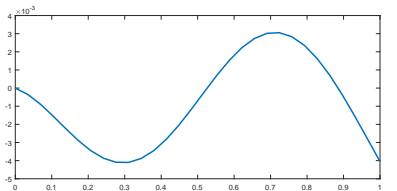

(d) Mode 4

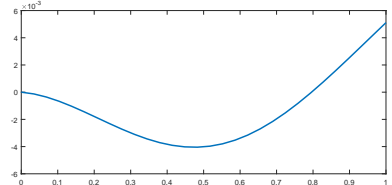

(b) Mode 2

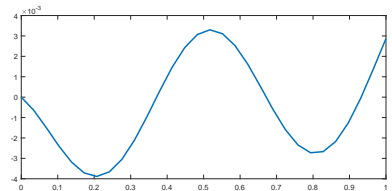

(e) Mode 5

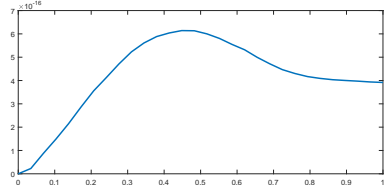

(c) Mode 3

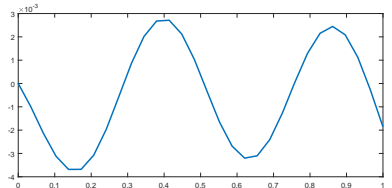

(f) Mode 6

Figure 7.12: Modal Shapes for Cantilever Beam 


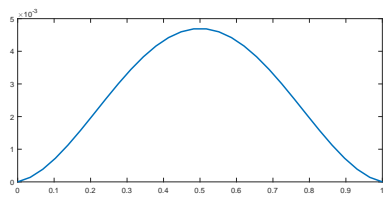

(a) Mode 1

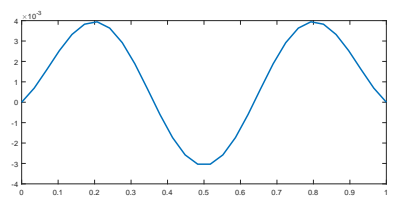

(d) Mode 4

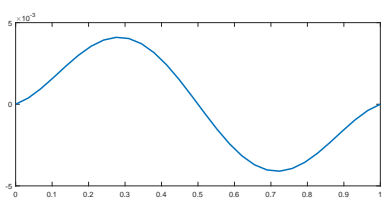

(b) Mode 2

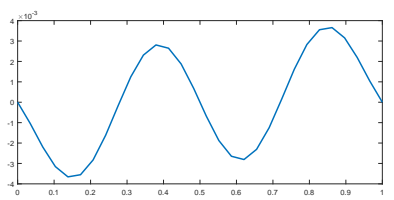

(e) Mode 5

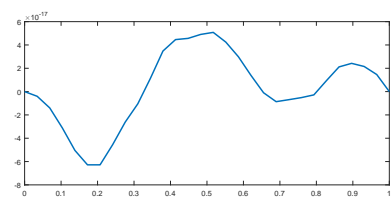

(c) Mode 3

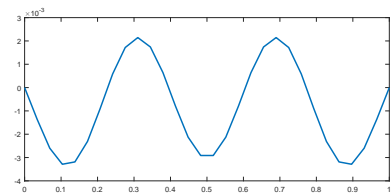

(f) Mode 6

Figure 7.13: Modal Shapes for a Fixed Ends Beam

\subsection{Time-stepping Methods for linear systems}

There are several time integrating methods for solving the equations of motion. Some of the most common are the Newmark- $\beta$ method (NBM), the Runge-Kutta method (RKM), the Central Difference method (CDM), and several more. For the scope of this project, the Newmark-Beta method, the Linear acceleration method and the Central Difference method are listed below. Three requirements are needed for selecting an appropriate numerical procedure: (1) convergence, (2) stability, and (3) accuracy [81].

\subsubsection{The Central Difference Method}

The CDM is based on a finite difference approximation of the time derivatives of displacement [81]. The expressions for velocity and acceleration are [81]

$$
\begin{array}{r}
\dot{u}_{i}=\frac{u_{i+1}-u_{i-1}}{2 \Delta t} \\
\ddot{u}_{i}=\frac{u_{i+1}-2 u_{i}+u_{i-1}}{(\Delta t)^{2}}
\end{array}
$$

Substituting these expressions for velocity and acceleration into the equation of motion gives [81]

$$
M\left[\frac{u_{i+1}-2 u_{i}+u_{i-1}}{(\Delta t)^{2}}\right]+C\left[\frac{u_{i+1}-u_{i-1}}{2 \Delta t}\right]+K u_{i}=p_{i}
$$


From time stepping, $u_{i}$ and $u_{i-1}$ are assumed known. Solving for $u_{i+1}$ gives

$$
\left[\frac{M}{(\Delta t)^{2}}+\frac{C}{2 \Delta t}\right] u_{i+1}=-\left[\frac{M}{(\Delta t)^{2}}-\frac{C}{2 \Delta t}\right] u_{i-1}+\left[\frac{2 M}{(\Delta t)^{2}}-K\right] u_{i}+p_{i}
$$

which may be written as

$$
A_{0} u_{i+1}=A_{1} u_{i}+A_{2} u_{i-1}+p_{i}
$$

where

$$
\begin{aligned}
& A_{0}=\left[\frac{M}{(\Delta t)^{2}}+\frac{C}{2 \Delta t}\right] \\
& A_{1}=\left[\frac{M}{(\Delta t)^{2}}-\frac{C}{2 \Delta t}\right] \\
& A_{2}=-\left[\frac{2 M}{(\Delta t)^{2}}+K\right]
\end{aligned}
$$

The unknown $u_{i+1}$ is given by

$$
u_{i+1}=\frac{A_{1} u_{i}+A_{2} u_{i-1}+p_{i}}{A_{0}}
$$

From Equation 7.30 it is evident that $u_{0}$ and $u_{-1}$ are needed to determine $u_{1}$. The initial displacement $u_{0}$ is known. For $i=0$

$$
\begin{array}{r}
\left(\dot{u_{0}}\right)=\frac{u_{1}-u_{-1}}{2 \Delta t} \\
\left(\ddot{u_{0}}\right)=\frac{u_{1}-2 u_{0}+u_{-1}}{(\Delta t)^{2}}
\end{array}
$$

solving for $u_{-1}$ from Equation 7.38 and substituting in Equation 7.39,

$$
u_{-1}=u_{0}-\Delta t\left(\dot{u}_{0}\right)+\frac{(\Delta t)^{2}}{2} \ddot{u}_{0}
$$

The equations of motion at time $\mathrm{t}=0$ shown in Equation 7.41 gives the acceleration in Equation 7.42,

$$
\begin{array}{r}
M \ddot{u}_{0}+C \dot{u}_{0}+K u_{0}=P_{0} \\
\ddot{u_{0}}=[M]^{-1}\left(P_{0}-C \dot{u_{0}}-K u_{0}\right)
\end{array}
$$


Based on the equations shown, the solution in the central difference method is explicit.

\section{Stability Condition}

The stability condition for the CDM is

$$
\frac{\Delta t}{T_{n}}<\frac{1}{\pi}
$$

In CDM, a much smaller time step is required to approach good accuracy. Rather, $\frac{\Delta t}{T_{n}}<0.1$ for definition of an adequate response, and $\Delta t=0.01$ to 0.02 to represent ground acceleration accurately [81].

\section{Computational Algorithm for Central Difference Method}

The computational algorithm of the MDOF CDM is shown in Box 7.3.1. The mass and damping matrix of the system are first obtained. Then the CDM method starts where the initial acceleration, velocity and displacement are calculated. The Gaussian AEM algorithm begins where the stresses and strains of each spring is calculated using the return mapping softening model, and the internal forces at each degree-of-freedom is calculated. The next time step begins where the new internal force is presented in the equations of motion and the new acceleration, velocity and displacement are calculated. 
Box. 7.3.1: Linear Central difference method

1. Initial Calculations

(a) Obtain Mass and Damping matrix of system

(b) $\ddot{u}_{0}=[M]^{-1}\left(P_{0}-C \dot{u}_{0}-K u_{0}\right)$ from [Eq. 7.42]

(c) Solve $u_{-1}=u_{0}-\Delta t\left(\dot{u}_{0}\right)+\frac{(\Delta t)^{2}}{2} \ddot{u}_{0}$ from [Eq. 7.40]

(d) select appropriate $\Delta t$

2. Calculate for each time step, $i=0,1,2,3, \ldots$

(a) update $p_{i}$ to include the new internal force vector

(b) $u_{i+1}=\frac{A_{1} u_{i}+A_{2} u_{i-1}+p_{i}}{A_{0}}$ from [Eq. 7.37]

(c) $\dot{u}_{i}=\frac{u_{i+1}-u_{i-1}}{2 \Delta t}$ and $\ddot{u}_{i}=\frac{u_{i+1}-2 u_{i}+u_{i-1}}{(\Delta t)^{2}} E q .[7.30]$

3. Repeat for the next time step. Replace $i$ by $i+1$

\subsubsection{The Newmark- $\beta$ Method}

The finite difference approximations for the Newmark- $\beta$ method developed by Newmark in 1959 are [82]

$$
\begin{array}{r}
u_{i+1}=u_{i}+\Delta t \dot{u}_{i}+\frac{1}{2} \Delta t^{2}\left[\left(1-\frac{\beta}{2}\right) \ddot{u}_{i}+2 \beta \ddot{u}_{i+1}\right] \\
\dot{u}_{i+1}=\dot{u}_{i}+\Delta t\left[(1-\gamma) \ddot{u}_{i}+\gamma \ddot{u}_{i+1}\right]
\end{array}
$$

The parameters $\beta$ and $\gamma$ represent the variation of acceleration over a time step and determine the stability and accuracy of the method [81]. $\gamma$ is typically $\frac{1}{2}$ and $\frac{1}{6}<\beta<\frac{1}{2}$. For linear systems, the equations of motion with iterations is

$$
M \ddot{u}_{i+1}+C \dot{u}_{i+1}+K u_{i+1}=p_{i+1}
$$

Rewriting Equation 7.44 to represent $\ddot{u}_{i+1}$ gives Equation 7.47. Substituting Equation 7.47 in 7.45 gives 7.48 .

$$
\begin{array}{r}
\ddot{u}_{i+1}=\frac{1}{\beta \Delta t^{2}}\left(u_{i+1}-u_{i}\right)-\frac{1}{\beta \Delta t} \dot{u}_{i}-\left(\frac{1}{2 \beta}-1\right) \ddot{u}_{i} \\
\dot{u}_{i+1}=\frac{\gamma}{\beta \Delta t}\left(u_{i+1}-u_{i}\right)+\left(1-\frac{\gamma}{\beta}\right) \dot{u}_{i}+\Delta t\left(1-\frac{\gamma}{2 \beta}\right) \ddot{u}_{i}
\end{array}
$$


Regrouping and solving for $u_{i+1}$, by substituting Equations 7.47 and 7.48 into Equation 7.46 gives

$$
\begin{array}{r}
M\left(\frac{1}{\beta \Delta t^{2}}\left(u_{i+1}-u_{i}\right)-\frac{1}{\beta \Delta t} \dot{u}_{i}-\left(\frac{1}{2 \beta}-1\right) \ddot{u}_{i}\right)+ \\
C\left(\frac{\gamma}{\beta \Delta t}\left(u_{i+1}-u_{i}\right)+\left(1-\frac{\gamma}{\beta}\right) \dot{u}_{i}+\Delta t\left(1-\frac{\gamma}{2 \beta}\right) \ddot{u}_{i}\right)+K u_{i+1}=p_{i+1}
\end{array}
$$

Rewriting Equation 7.49 in terms of the displacement, velocity and acceleration gives

$$
\begin{array}{r}
u_{i+1}\left(\frac{M}{\beta \Delta t^{2}}+\frac{C \gamma}{\beta \Delta t}+K\right)+u_{i}\left(-\frac{M}{\beta \Delta t^{2}}-\frac{C \gamma}{\beta \Delta t}\right) \\
+\dot{u}_{i}\left(-\frac{M}{\beta \Delta t}+C\left(1-\frac{\gamma}{\beta}\right)\right)+\ddot{u}_{i}\left(-M\left(\frac{1}{2 \beta}-1\right)+C \Delta t\left(1-\frac{\gamma}{2 \beta}\right)\right)=p_{i+1}
\end{array}
$$

which can be written in the form of

$$
A_{0} u_{i+1}=A_{1} u_{i}+A_{2} \dot{u}_{i}+A_{3} \ddot{u}_{i}+p_{i}
$$

where

$$
\begin{array}{r}
A_{0}=\left(\frac{M}{\beta \Delta t^{2}}+\frac{C \gamma}{\beta \Delta t}+K\right) \\
A_{1}=\left(\frac{M}{\beta \Delta t^{2}}+\frac{C \gamma}{\beta \Delta t}\right) \\
A_{2}=\left(\frac{M}{\beta \Delta t}+C\left(\frac{\gamma}{\beta}-1\right)\right) \\
A_{3}=\left(M\left(\frac{1}{2 \beta}-1\right)+C \Delta t\left(\frac{\gamma}{2 \beta}-1\right)\right)
\end{array}
$$

The displacement at time $i+1$ is

$$
u_{i+1}=\left[A_{0}\right]^{-1}\left[A_{1} u_{i}+A_{2} \dot{u}_{i}+A_{3} \ddot{u}_{i}+p_{i}\right]
$$

and the acceleration can also be obtained from the equation of motion 7.46

$$
\ddot{u}_{i+1}=[M]^{-1}\left[p_{i+1}-C \dot{u}_{i+1}-K u_{i+1}\right]
$$


Special cases of the Newmark- $\beta$ method arise for specific values of $\gamma$ and $\beta$. For $\gamma=1 / 2$ and $\beta=1 / 4$, the Newmark method is identical to the constant average acceleration. For $\gamma=1 / 2$ and $\beta=1 / 6$, the Newmark method is identical to the linear acceleration. Finally, for $\gamma=1 / 2$ and $\beta=0$ the Newmark method is reduced to the central difference method. For $\gamma=1 / 2$ the Newmark method is at least second-order accurate. It is first order accurate for all other values of $\gamma$ [83]. If the residual nonlinear forces are not negligible, then Equation 7.46 must be solved using the Newton-Raphson method.

\section{Newmark Method Stability}

The Newmark's method is stable with the condition of Equation 7.58 [81],

$$
\frac{\Delta t}{T_{n}} \leq \frac{1}{\pi \sqrt{2}} \frac{1}{\sqrt{\gamma-2 \beta}}
$$

For $\gamma=\frac{1}{2}$ and $\beta=\frac{1}{4}$, (constant average acceleration method), the condition becomes

$$
\frac{\Delta t}{T_{n}}<\infty
$$

Equation 7.59 indicates that the constant average acceleration method is stable for any $\Delta t$. It is only accurate for small values of $\Delta t$. For $\gamma=\frac{1}{2}$ and $\beta=\frac{1}{6}$, the linear acceleration method is stable if

$$
\frac{\Delta t}{T_{n}} \leq 0.551
$$

\section{Computational Algorithm}

The computational algorithm for the linear multiple degree-of-freedom Newmark Method for a system is shown in Box 7.3.2. 


\section{Box. 7.3.2: MDOF Linear Newmark Method}

1. Initial Calculations

(a) Solve $M \ddot{u}_{0}+C \dot{u}_{0}+K u_{0}=p_{0}$ for $\ddot{u}_{0}[E q$. 7.46]

(b) Select $\Delta t$

(c) Calculate $A_{1}, A_{2}, A_{3}$ from [Eq.7.53,7.54,7.55]

2. Calculate for each time step, $i=0,1,2,3, \ldots$

(a) Obtain $u_{i+1}=\frac{A_{1} u_{i}+A_{2} \dot{u}_{i}+A_{3} \ddot{u}_{i}+p_{i}}{A_{0}}$ [Eq. 7.56]

(b) Obtain $\dot{u}_{i+1}=\frac{\gamma}{\beta \Delta t}\left(u_{i+1}-u_{i}\right)+\left(1-\frac{\gamma}{\beta}\right) \dot{u}_{i}+\Delta t\left(1-\frac{\gamma}{2 \beta}\right) \ddot{u}_{i}[E q$. $7.48]$

(c) Obtain $\ddot{u}_{i+1}=\frac{1}{\beta \Delta t^{2}}\left(u_{i+1}-u_{i}\right)-\frac{1}{\beta \Delta t} \dot{u}_{i}-\left(\frac{1}{2 \beta}-1\right) \ddot{u}_{i}[E q .7 .47]$

3. Repeat for the next time step. Replace $i$ by $i+1$

\subsubsection{Numerical Methods Classifications}

The general classifications of numerical integration methods are: explicit and implicit [83]. For single step explicit methods, the displacements and velocities at $t_{i+1}$ are determined in the closed form from the displacements, velocities and accelerations at $t_{i}$. Hence, for structural systems with linear elastic stiffness and damping, the discrete-time systems can be written as Equation 7.61.

$$
\left[\begin{array}{c}
u\left(t_{i+1}\right) \\
\dot{u}\left(t_{i+1}\right)
\end{array}\right]=A\left[\begin{array}{c}
u\left(t_{i}\right) \\
\dot{u}\left(t_{i}\right)
\end{array}\right]+B f^{e x t}\left(t_{i}\right)
$$

where $A$ is a $2 n \times 2 n$ discrete time dynamics matrix that depends on $M, C, K, \Delta t$, and the time step. However, the implicit methods are a solution of a set of linear algebraic equations at each time step [83].

Based on the equations shown, the solution in Newmark method is implicit, and the CDM is explicit. In the CDM no iterative procedure is required because all the constitutive variables are available from computations at previous time steps [84]. 


\subsection{Time-stepping methods for Nonlinear Sys- tems}

The implementation of nonlinear systems in the Equations of motion is simply done by using internal forces rather than the stiffness multiplied by the displacement. Similar to the amended equation of motion for nonlinearity (Equation 7.13), this modification is applied to the time integration schemes.

$$
M \ddot{u}+C \dot{u}+f(s)=p(t)
$$

The modifications to the Nonlinear Newmark- $\beta$ Method and the Central Difference Method are presented in the following sections.

\subsubsection{Nonlinear Newmark- $\beta$ Method}

The equations of the Newmark- $\beta$ method were derived in section 7.3.2. The acceleration, velocity and displacements equations do not change; however the equations of motion 7.62 is implemented, where the stiffness matrix multiplied by the displacements is replaced by the internal forces. The iteration scheme that will be used is the Newton-Raphson method, for static analysis of a nonlinear system. For the equations of motion written in the form of Equation 7.63, only $A_{0}$ changes since the stiffness matrix is not incorporated. The modified $A_{0}$ is shown in Equation 7.64 .

$$
\begin{array}{r}
A_{0} u_{i+1}=A_{1} u_{i}+A_{2} u_{i-1}+p_{i}-f_{S i} \\
A_{0}=\left(\frac{M}{\beta \Delta t^{2}}+\frac{C \gamma}{\beta \Delta t}\right)
\end{array}
$$

It should be noted that this method determines the solution at time $(i+1)$ from the equilibrium condition at time $(i+1)$. The resisting force $\left(f_{S}\right)_{(i+1)}$ is an implicit function of the unknown $u_{(i+1)}$, therefore an iteration method is required [81]. 


\section{Newton Raphson Iteration Scheme}

An iterative procedure is used such that $u^{j+1}$ can be found as an improved estimate from $u^{j}$. Expanding the resisting force $f_{S}^{(j+1)}$ in Taylor series about the known estimate $u^{j}$ gives

$$
f_{S}^{(j+1)}=F_{S}^{j}=\left.\frac{\partial f_{S}}{\partial u}\right|_{u^{j}}\left(u^{(j+1)}-u^{(j)}\right)+\left.\frac{1}{2} \frac{\partial^{2} f_{S}}{\partial u^{2}}\right|_{u^{j}}\left(u^{(j+1)}-u^{(j)}\right)^{2}+\ldots
$$

If $u^{j}$ is close to the solution then change $\Delta u^{j}=u^{(j+1)}-u^{j}$ will be small and the second higher order terms are neglected, leading to Equation 7.66

$$
\begin{array}{r}
f_{S}^{j+1}=f_{S}^{j}+K_{T}^{j} \Delta u^{j}=0 \\
K_{T}^{j} \Delta u^{j}=p-f_{S}^{j}=R^{j}
\end{array}
$$

where $K_{T}$ is the tangent stiffness at $u^{j}$. Solving 7.67 gives a new estimate of the displacement

$$
u^{(j+1)}=u^{(j)}+\Delta u^{(j)}
$$

The additional displacement $\Delta u^{(j+1)}$ due to the residual force is calculated from

$$
K_{T}^{(j+1)} \Delta u^{(j+1)}=R^{(j+1)}
$$

The new displacement is

$$
u^{(j+2)}=u^{(j+1)}+\Delta u^{(j+1)}
$$

The new value of $R^{(j+2)}$ is continued until convergence is achieved.

\section{Computational Algorithm}

The new computational algorithm for the nonlinear Newmark- $\beta$ method is presented in Box 7.4.1. 


\section{Box. 7.4.1: MDOF Nonlinear Newmark method with Newton}

\section{Raphson iteration}

1. Initial Calculations

(a) Solve $M \ddot{u}_{0}+C \dot{u}_{0}+f(s)_{0}=p_{0}$ for $\ddot{u}_{0} E q \cdot[7.46]$

(b) Select $\Delta t$

(c) Calculate $A_{1}, A_{2}, A_{3}$ from [Eq.7.53,7.54,7.55]

2. Calculate for each time step, $i=0,1,2,3, \ldots$

(a) initialize $j=1, u_{i+1}^{j}=u_{i},\left(f_{s}\right)_{i+1}^{j}=\left(f_{s}\right)_{i}$ and $\left(K_{T}\right)_{i+1}^{j}=\left(K_{T}\right)_{i}$

(b) $\hat{p}_{i}=A_{1} u_{i}+A_{2} \dot{u}_{i}+A_{3} \ddot{u}_{i}+p_{i}$

3. For each Newton Raphson iteration, $j=1,2, \ldots$

(a) Calculate residual force vector $\hat{R}_{i+1}^{j}$

(b) check if convergence is obtained. if convergence criteria is not met then continue to step 3c, otherwise go to step 4

(c) $\left(\hat{K}_{T}\right)_{i+1}^{j}=\left(K_{T}\right)_{i+1}^{j}+A_{1}$

(d) $\Delta u_{i+1}^{j+1}=\hat{R}_{i+1}^{j} /\left(\hat{K}_{T}\right)_{i+1}^{j}$

(e) $u_{i+1}^{j+1}=i+1^{j}+\Delta u^{(j)}$

Replace $j$ by $j+1$, and repeat steps 3 a to $3 \mathrm{e}$.

4. Calculate velocity and acceleration

(a) Obtain $\dot{u}_{i+1}$ from [Eq. 7.48$]$

(b) Obtain $\ddot{u}_{i+1}$ from [Eq. 7.47]

5. Repeat for the next time step $i$. Replace $i$ by $i+1$

\subsubsection{Nonlinear Central Difference Method}

Rewriting the equations of motion from Equation 7.73 to incorporate the inelastic behaviour gives

$$
\begin{gathered}
M \ddot{u}_{i}+C \dot{u}_{i}+\left(f_{S}\right)_{i}=P_{i} \\
\ddot{u}_{i}=[M]^{-1}\left(P_{i}-C \dot{u}_{i}-\left(f_{S}\right)_{i}\right) \\
M\left[\frac{u_{i+1}-2 u_{i}+u_{i-1}}{(\Delta t)^{2}}\right]+C\left[\frac{u_{i+1}-u_{i-1}}{2 \Delta t}\right]+f_{S i}=p_{i}
\end{gathered}
$$


SO

$$
A_{0} u_{i+1}=A_{1} u_{i}+A_{2} u_{i-1}+p_{i}-f_{S i}
$$

where

$$
\begin{array}{r}
A_{0}=\left[\frac{M}{(\Delta t)^{2}}+\frac{C}{2 \Delta t}\right] \\
A_{1}=\left[\frac{M}{(\Delta t)^{2}}-\frac{C}{2 \Delta t}\right] \\
A_{2}=-\left[\frac{2 M}{(\Delta t)^{2}}\right]
\end{array}
$$

The difference between the linear and nonlinear CDM is in Equation 7.77, where the stiffness matrix $K$ is no longer a part of the equation. The advantage is that $K$ is no longer needed to be inverted in every iteration. Since the method will be applied to a MDOF system of high rise structures, this will significantly reduce computational cost as opposed to using the Newmark- $\beta$ method. Another advantage is that $f_{(S i)}$ is calculated explicitly, making the method one of the simplest in comparison to other MDOF procedures for analysis. The computational algorithm is therefore amended respectively. A disadvantage of using the CDM is that the time step must be really small. Considering that the mass and damping matrix are diagonalised, having a small time step is not so disadvantageous since the computational cost is quite low, and there is no stiffness matrix inversion. A special case of the dynamic problem can result in convergence of solution of a static problem. In other words, a static problem can be presented as a special case of dynamic problem. For representing the static case using the dynamic model, $K u=F_{\text {int }}$. The mass matrix, and damping matrix of Equation 7.19 are presented in the following sections.

\subsubsection{Summary of Methods}

The method chosen for the nonlinear dynamic analysis is based on a comparison of the methods, shown in Table 7.1. Assuming a cantilever beam, with span of $1 \mathrm{~m}$, and $0.15 \mathrm{~m}$ cross-section, the calculated critical time step for the Central Average Acceleration Method, $\Delta t$ can be any number, however it must be small for 
Table 7.1: Summary of advantages and disadvantages

\begin{tabular}{r||l|l} 
& Newmark- $\beta$ & Central Differences \\
\hline \hline Numerical method & Implicit & Explicit \\
\hline critical $\Delta t$ & Stable with large $\Delta t$ & Stable at small $\Delta t$ \\
\hline Nonlinearity adaptation & Requires iteration & No iteration scheme \\
& scheme such as the & required \\
& Newton Raphson & \\
& method & \\
& Requires inverting the & does not require in- \\
& stiffness matrix in ev- & verting the stiffness \\
& ery iteration & matrix
\end{tabular}

obtaining desired accuracy, and for the CDM should be at least $\Delta t=1.6 \times 10^{-4}=$ 0.00016 .

\subsection{Dynamic Formulation and Application in AEM}

Now that the elastic and inelastic dynamic equations are formulated, they will be applied in the context of AEM to analyse structural systems subject to dynamic loading. The mass and damping matrix are formulated in the following sections. Application of the dynamic analysis to earthquake loading and extreme wind loading is presented in the following chapters.

\subsubsection{Determination of Mass Matrix}

The mass matrix for a single element is a $3 \times 3$ matrix, since each element has 3 degrees-of-freedom. The mass matrix is a diagonal matrix, with the diagonal elements shown in Equation 7.78. The element mass and inertia are assumed lumped at the element centre. The mass matrices must comply with the following conditions [85]: (1) symmetry: $\left(M^{e}\right)^{T}=M^{e}$; (2) conservation; and (3) positivity: the matrix should have diagonally positive elements.

$$
M^{e}=\operatorname{diag}\left\{\begin{array}{c}
M_{1} \\
M_{2} \\
M_{\alpha}
\end{array}\right\}=\operatorname{diag}\left\{\begin{array}{c}
m / 2 \\
m / 2 \\
\alpha m l^{2}
\end{array}\right\}
$$


where, $\left[M_{1}\right]$ and $\left[M_{2}\right]$ are the element mass in the $\mathrm{x}$ direction and the $\mathrm{y}$-direction respectively, and $\left[M_{3}\right]$ is the mass due to the rotation direction , $m$ is the total mass of the element, $\alpha$ is a small value and $l$ is the length of the element. In the computational algorithm, the mass matrix is:

1. localized: mass matrix of each element is formed separately

2. transformed from local to global coordinates: $M_{g}=T^{t} M T$

3. assembled into global matrix with all elements and degrees-of-freedom

\subsubsection{Determination of Damping Matrix}

The process by which vibration diminishes in amplitude is damping [81]. The Rayleigh Damping is a proportional damping model that expresses the damping as a linear combination of mass and stiffness, shown as Equation 7.79.

$$
\mathbf{C}=a \mathbf{M}+b \mathbf{K}
$$

where $a$ and $b$ are damping parameters, $[\mathbf{C}]$ is the damping matrix, $[\mathbf{M}]$ is the mass matrix, and $[\mathbf{K}]$ is the stiffness matrix.

\section{$a$ and $b$ calculation}

For linear problems the natural frequencies $\omega_{i}$ and the modes of vibration $\phi_{i}$ can be obtained. The damping ratio for the $i^{\text {th }}$ mode of a system is

$$
\xi_{i}=\frac{a}{2 \omega_{i}}+\frac{b \omega_{i}}{2}
$$

where, $\xi_{i}$ is the damping ratio, and $\omega_{i}$ is the natural frequency. For every $\omega_{i}$ there is a corresponding $\xi_{i}$. Two values of $\xi_{i}$ can be set to solve the two equations with two unknowns $a$ and $b$ using Equation 7.80. Once $a$ and $b$ are calculated the damping matrix $\mathrm{C}$ can be calculated.

It is evident that with an increasing $\omega_{i}$, then $\xi_{i}$ will get bigger. However this is not significant since the larger $\omega_{i}$ are resulted from higher degrees of modal shapes which are considered insignificant. For simplicity of the dynamic analysis using the Gaussian AEM, $b$ is chosen as zero. So the damping matrix is calculated 
as $C=a M$.

\subsubsection{Implementing dynamics and nonlinearity in MAT- LAB code}

Implementing the nonlinear time stepping schemes into the dynamic code is presented. The nonlinearity in the model comes from the spring material nonlinearity and is determined by whether the material is elastic or elasto-plastic, which is related to the softening model yield criteria. Including the softening model along with the Central Difference method allows to generate a code with both linear and nonlinear behaviour. A few modifications are required to be done to the return mapping softening algorithm for implementing the CDM. Firstly, the stiffness matrix is not needed to be updated with every iteration, since the dynamic model does not require the stiffness matrix, rather only the internal forces need to be calculated. Therefore, the stiffness matrix is not needed to be calculated in every iteration. Secondly, since the CDM is an explicit method the Newton Raphson iteration scheme is not needed. Considering that there is no stiffness matrix update needed or inversion, and no Newton Raphson scheme, this leads to a computationally low cost algorithm.

The computational algorithm for a nonlinear dynamic code with AEM is explained for each time step. At the first time step an initial displacement velocity and acceleration are calculated from an initial guess $(u=0)$. Then the Gaussian AEM with softening material behaviour begins. There is a loop for every element. For each element, the stresses and strains for each spring are calculated from the displacement calculated using the CDM. Based on the calculated stresses and strains the yield criteria for each spring is examined. The yield criteria determines whether the springs are elastic, elasto-plastic or failed, and then the corresponding internal forces are calculated. For failed springs, the internal force is set to be zero. The internal forces for each springs are assembled to the global force vector to be used in the next time step iteration. At the next time step the internal forces are updated, as well as all other external forces for that time step, and the new displacement, velocity and acceleration are calculated using the CDM. Then the Gaussian AEM algorithm begins again. This process is repeated until the 
time required is reached. The computational algorithm is displayed in Box 7.5.3.

\section{Box. 7.5.1: Nonlinear CDM with Gaussian AEM}

1. Initial Calculations

(a) Obtain Mass and Damping matrix of system

(b) $\ddot{u}_{i}=[M]^{-1}\left(P_{i}-C \dot{u}_{i}-\left(f_{S}\right)_{i}\right)$ Eq.[7.72]

(c) Solve $u_{-1}=u_{0}-\Delta t\left(\dot{u}_{0}\right)+\frac{(\Delta t)^{2}}{2} \ddot{u}_{0}[E q$. 7.40]

(d) Calculate $A_{0}, A_{1}$ and $A_{2}$ [Eq. 7.75, 7.76, 7.77]

(e) select appropriate $\Delta t$

2. Calculate for each time step, $i=0,1,2,3, \ldots$

(a) $u_{i+1}=[A]^{-1}\left[A_{1} u_{i}+A_{2} u_{i-1}+p_{i}-f_{S i}\right]$ Eq.[7.74]

(b) $\dot{u}_{i}=\frac{u_{i+1}-u_{i-1}}{2 \Delta t}$ and $\ddot{u}_{i}=\frac{u_{i+1}-2 u_{i}+u_{i-1}}{(\Delta t)^{2}} E q$. [7.30]

(c) Begin Gaussian AEM loop

- Loop through every element and then spring to calculate the new stresses and strains from $u_{i+1}$, using return mapping algorithm and softening yield criteria.

- Update internal force vector $f_{S i}$.

3. Repeat from 2 for the next time step. Replace $i$ by $i+1$

\subsection{Dynamic Analysis for Verification}

This section presents some implementation of the dynamic model to simple structures. Analysis of structures undergoing free vibration with and without damping are presented to ensure that the model behaves as expected. A large displacement analysis of a frame is then presented, where no element separation is allowed.

\subsubsection{Free Vibration}

Free vibration is the motion of a structure without an external forces or dynamic excitation [81]. The vibration is initiated by an initial disturbance to the structure. This phenomenon is verified in the AEM algorithm presented. Consider a cantilever beam with a load applied at the free end at time step 500 $(\Delta t=0.00005$, time $=0.025 \mathrm{sec}$, iteration 500 out of 10,000$)$. The free vibration of the undamped and damped system are presented as follows. 


\section{Undamped system}

For an undamped system the expected behaviour of the beam after a disturbance is shown in Figure 7.14. The equations of motion governing the undamped system are presented in Equation 7.81, with initial conditions in Equation 7.82.

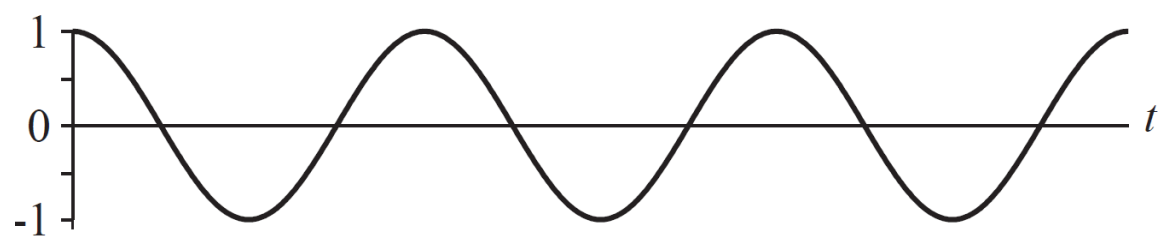

Figure 7.14: Free vibration of undamped system [81]

$$
\begin{array}{r}
M \ddot{u}+K u=0 \\
u=u(0) \quad \dot{u}=\dot{u}(0)
\end{array}
$$

Figure 7.15 shows the displacement, velocity and acceleration of the point at which the load is applied. Figure $7.15 \mathrm{a}$ is the displacement, velocity and acceleration of the free end of the system during the first 0.2 seconds. As can be seen before the perturbation was presented in the $500^{\text {th }}$ iteration, the displacement, velocity and acceleration is zero. From Figure $7.15 \mathrm{~b}$ the graphs show that the system is vibrating freely with no damping occurring. The results show good accordance with the expected result from Figure 7.14.
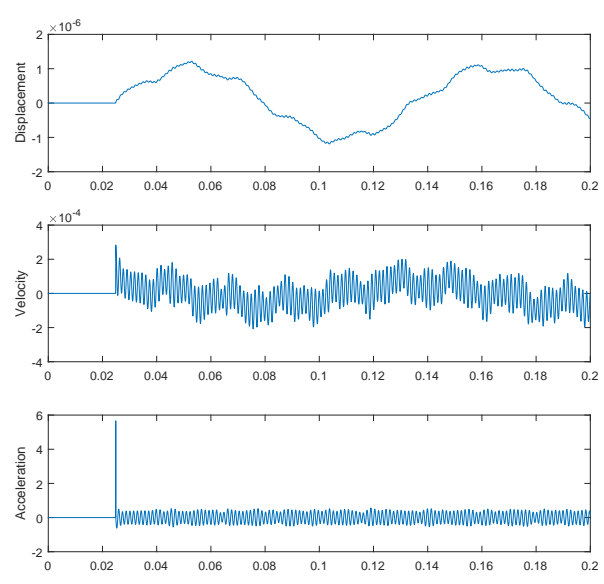

(a) 0.2 seconds
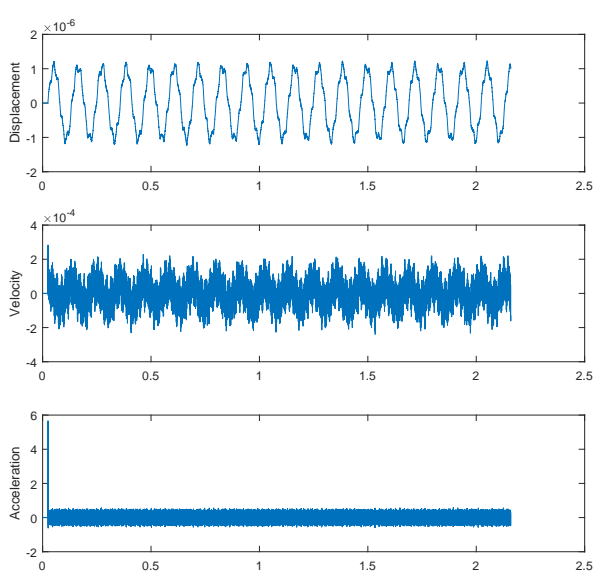

(b) 2.25 seconds

Figure 7.15: Free vibration of point at which load is applied 


\section{Damped system}

The free vibration of a damped system is shown in Figure 7.16, where the vibration eventually "damps" out due to the damping coefficient in the system. Similar to the example in the undamped system, a cantilever beam is disturbed at the $500^{\text {th }}$ time step, and the displacement, velocity and acceleration are shown in Figure 7.17. It is evident that leaving the system to react to the disturbed force eventually leads to a static solution.

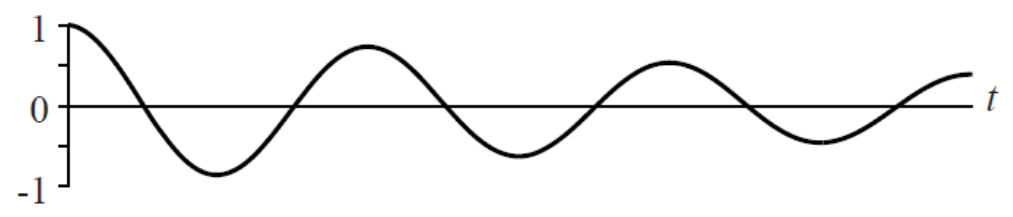

Figure 7.16: Free vibration of damped system [81]

$$
M \ddot{u}+C \dot{u}+K u=0
$$
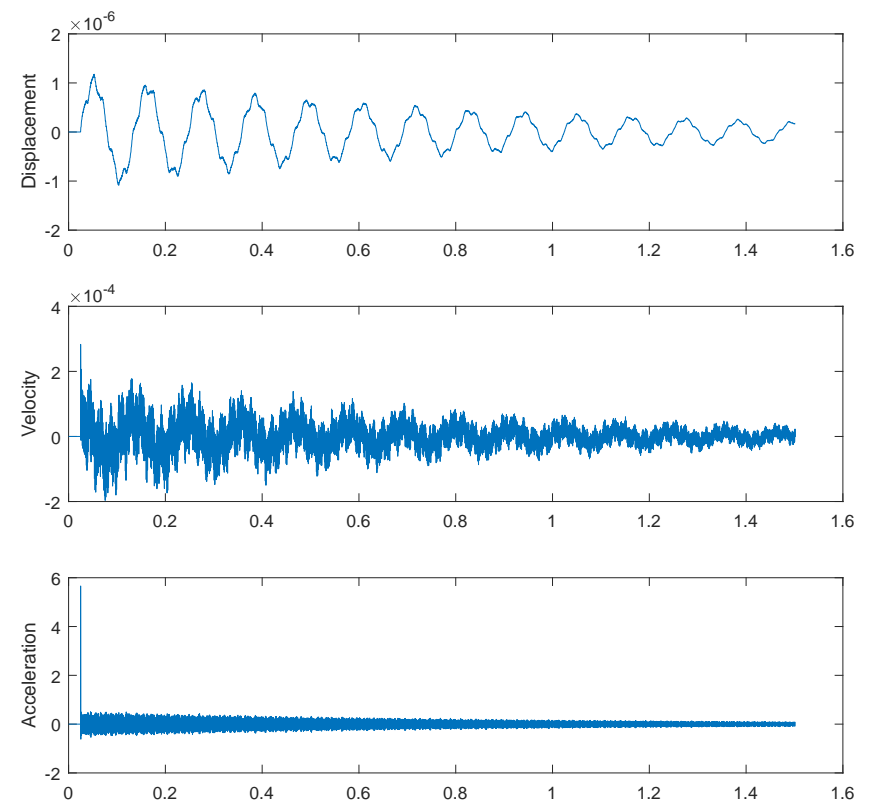

Figure 7.17: Displacement, velocity and acceleration of damped cantilever system at free end 


\subsection{Analysis Results}

Different types of loading scenarios are implemented on beams and frames in this section to demonstrate the dynamic behaviour of a multiple degree-of-freedom system. Considering a cantilever beam with $1 \mathrm{~m}$ span and $0.15 \times 0.15 \mathrm{~m}$ crosssection. The material properties are similar to the beam shown from Table 5.1. Different loading conditions are are applied to the beam as shown below.

\section{Point load increasing with time}

Figure 7.18a displays the force-displacement diagram of the free end of the beam, where the load is applied. Figure 7.18b shows the displacement, velocity and acceleration of the point at which the load is applied, with respect to time. The Figure shows that the displacement, velocity and acceleration damp out to eventually a static solution. Figure 7.19 is the deflection of the beam at different time steps. It is cleat that the deflection is oscillating between the positive and negative y direction, which is expected in the dynamic behaviour.

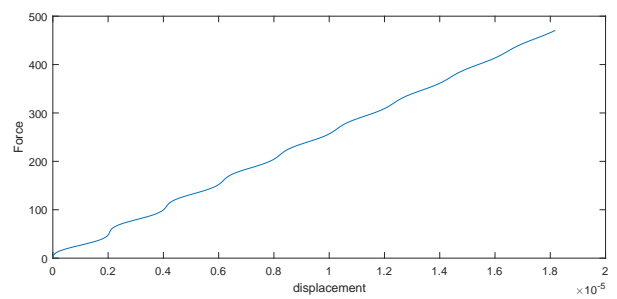

(a)

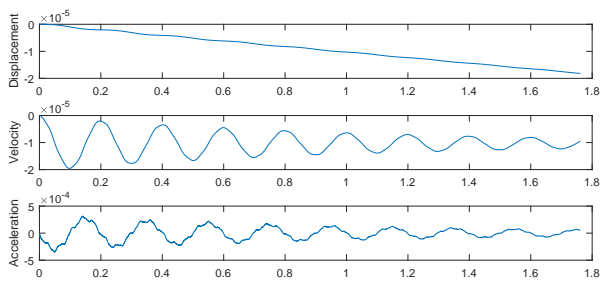

(b)

Figure 7.18: displacement, velocity and acceleration at free end

Figure 7.19 shows the behaviour of the beam deflection at different time steps.

\section{Point load on cantilever beam with no damping}

The acceleration velocity and displacement of the cantilever beam with no damping is shown in Figure 7.20. The results show that velocity and acceleration are clearly undamped. The displacement increases with time (in the negative direction) because the load is incrementally increasing. 


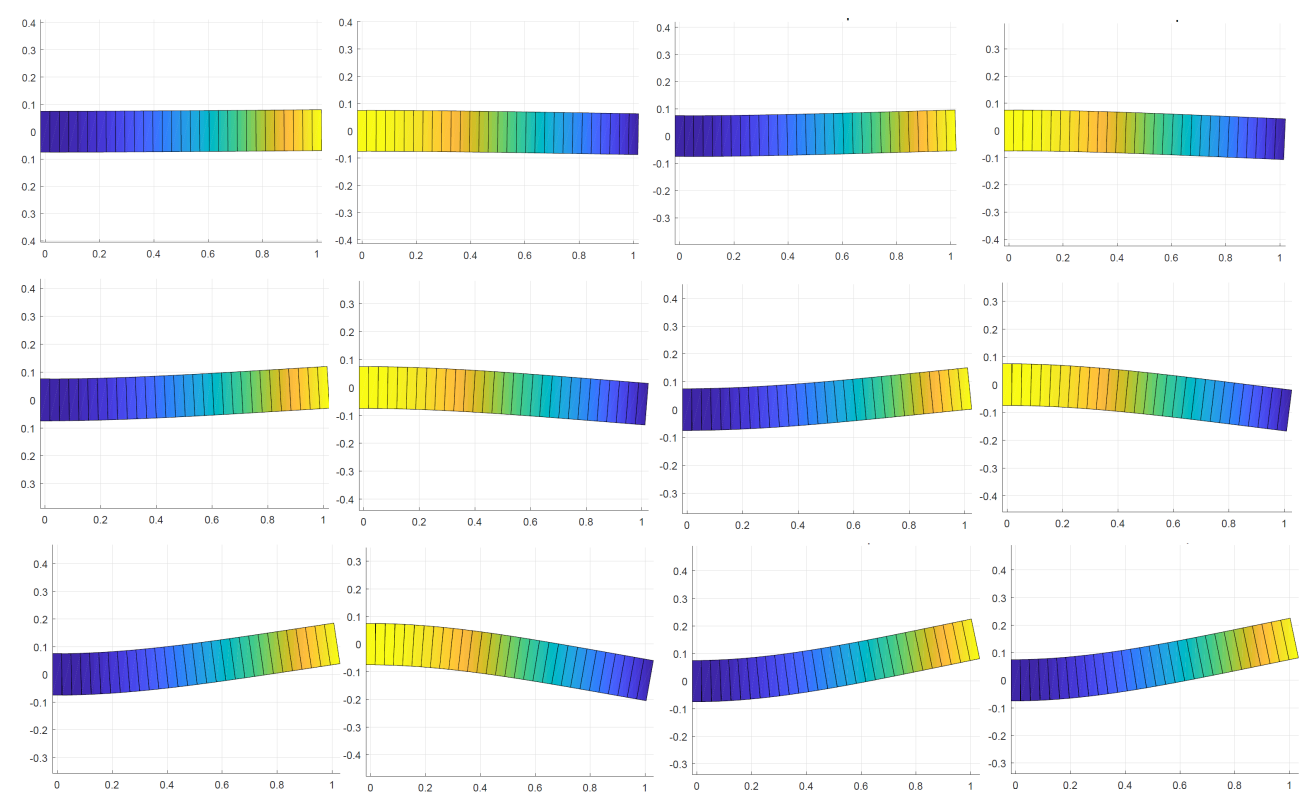

Figure 7.19: Beam Deflection of cantilever beam at different time steps
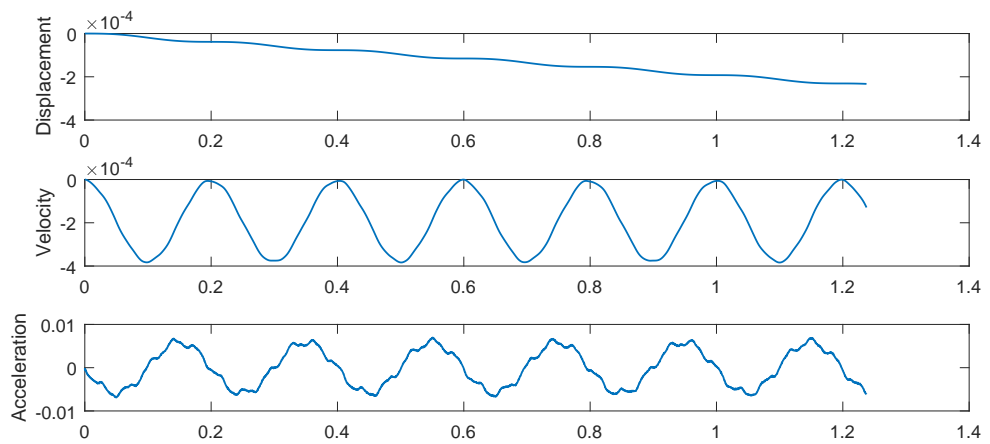

Figure 7.20: Displacement, velocity and acceleration of free free end of cantilever beam for undamped system

\section{Point load increasing with time applied sinusoidally}

The cantilever beam is subject to an incrementally increasing sinusoidal load, shown in Equation 7.84. Figure 7.21 is the force displacement diagram of the free end of the cantilever beam, subjected to the loading. Figure 7.22 shows the displacement, velocity and acceleration versus the time. The sinusoidal behaviour is clearly shown.

$$
F_{\text {ext }}=F(t) \times \sin \left(\omega_{n} t\right)
$$




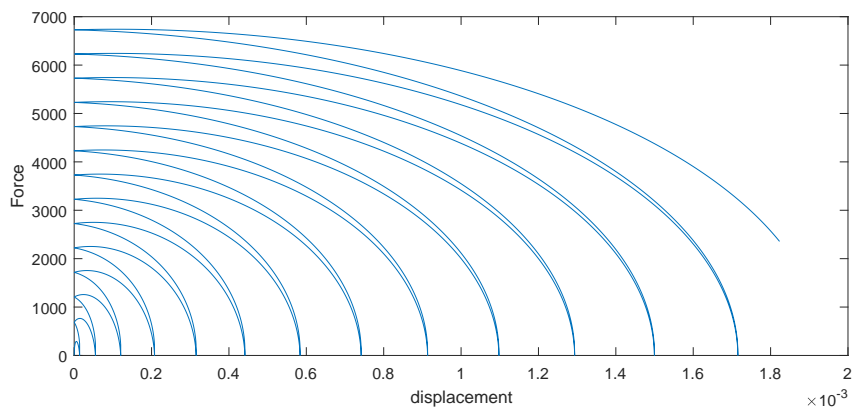

Figure 7.21: Force-displacement diagram for sin load
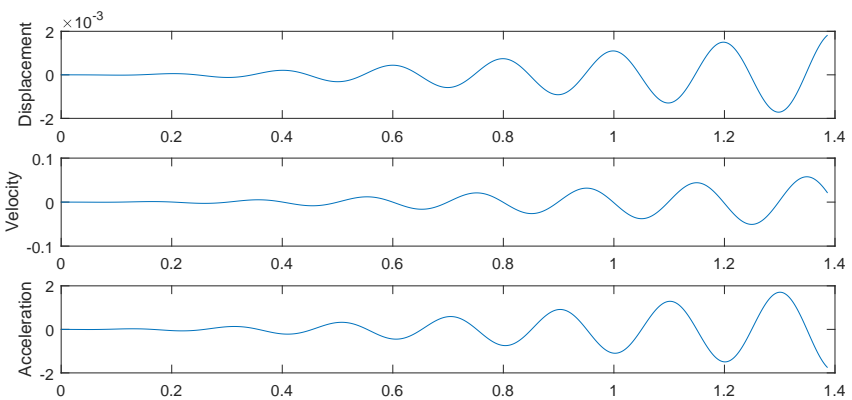

Figure 7.22: Displacement, velocity and acceleration at free end for cantilever beam with sin load

\section{Large displacement Analysis}

The dynamic analysis is applied to a multi-storey frame. In this example no failure (element separation) between elements is considered. So if the structure is subjected to large loads then unrealistic large displacements are expected. The deflection of a column frame subject to a large load that results in large displacement is presented. The frame has material properties of steel and is loaded at the top left point in the $\mathrm{x}$-direction with an incrementally increasing load. The softening material model is used and the structure is analysed dynamically using the nonlinear CDM. The structure undergoes permanent deformation since the load is very large plastic deformation was found in most elements. The unfactored deflection of the frame is shown in Figure 7.23. As can be seen from the deflection figures, at time 1.8 seconds, the frame already had a deflection of approximately $6 \mathrm{~m}$ at the top roof, which is unrealistic. The large deformation of the structure is well presented. 

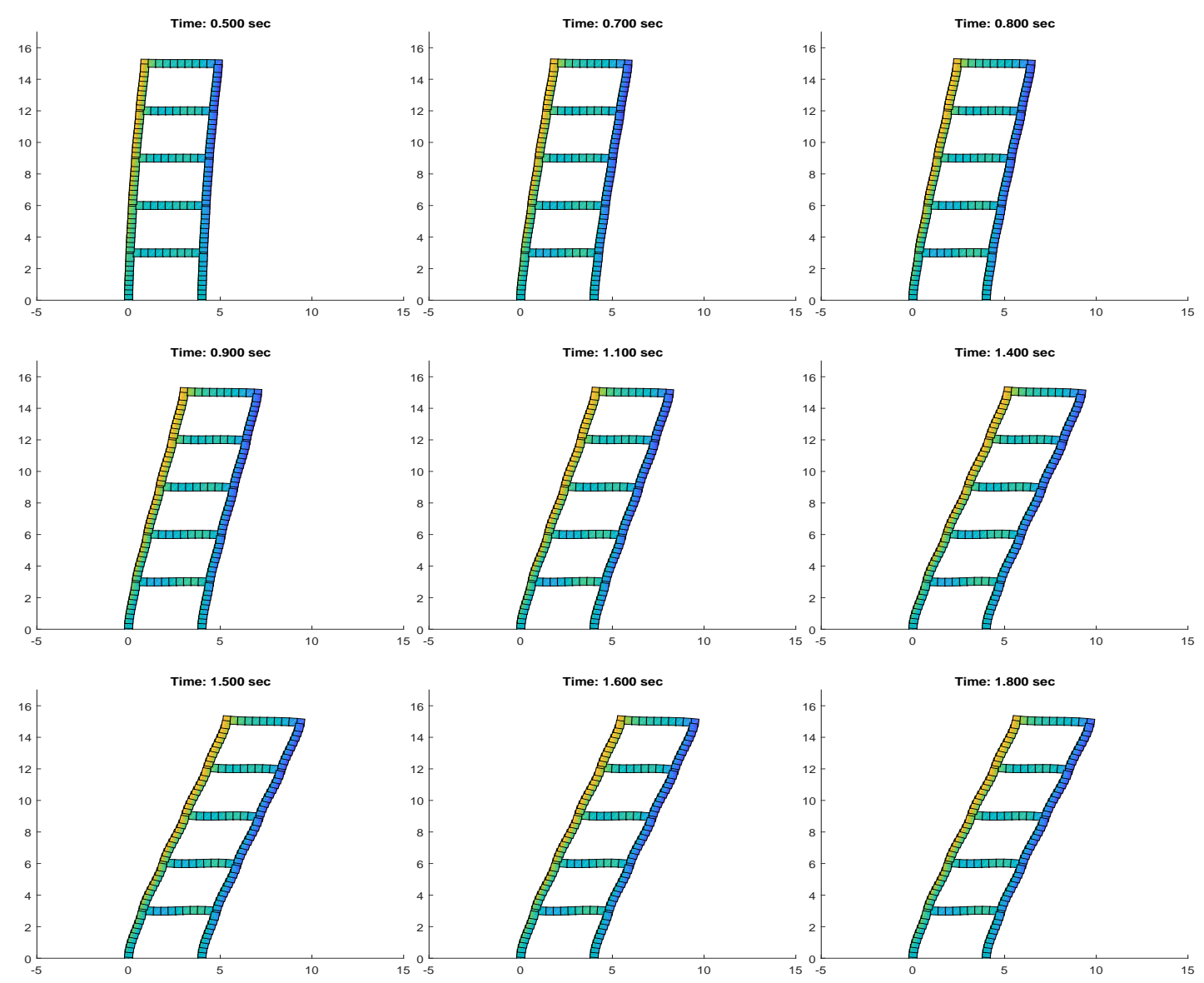

Figure 7.23: Large displacement for tall frame with no collapse

\subsection{Conclusion}

In this chapter a damage material model was presented for modelling softening material behaviour. The return mapping algorithm was formulated based on the 1D Hardening plasticity model. Material damage was represented as a material that experiences a softening behaviour. The return-mapping algorithm successfully modelled the behaviour, however it caused problems when springs exceeded the failed strain point. If a spring exceeds the failure limit then it would be considered totally detached from the system. In order to represent a failed spring, the stiffness of the spring is set as zero, rather than deleting the spring from the whole system. The problem is that zeros in the stiffness matrix cause the stiffness matrix to become singular, and cannot be inverted.

The use of a dynamic time integrator solved the problem of singularity in the stiffness matrix from the softening material behaviour. The Central Difference 
Method was chosen as the most suitable integrator, rather than the Newmark- $\beta$ Method. The CDM is explicit so does not require an iterative procedure to solve the dynamic equations. Although using the time step size required for the CDM is quite small, the advantages still outweigh this disadvantage. For simplicity, the damping matrix was chosen to be calculated using only the mass matrix. So the damping matrix is diagonal.

Some analysis of structures were presented such as the free vibration of a damped and undamped cantilever beam, analysis of cantilever beam with a sinusoidal load, and a large displacement analysis of a frame.

Since the damage material model and the failure of elements has been established in the Gaussian-AEM model the analysis of progressive collapse of structures can be presented. The collapse of frames subject to earthquake and wind loads are presented in the following chapters. 


\section{Chapter 8}

\section{Structures subject to Earthquake loads}

In this chapter, dynamic analysis of frames subject to earthquakes is presented. The purpose is to analyse simple frame structures and their reaction to recorded time-acceleration data of earthquakes that had occurred. Modern building codes have strict requirements on the design of buildings to resist earthquakes. This chapter will entail an introduction to how earthquakes occur and how they are measured. From the given data of previous earthquakes, the ground acceleration is applied to the frames and the response of the structures is analysed using the nonlinear dynamic Gaussian AEM. The modification to the AEM algorithm is presented along with some examples.

\subsection{Introduction}

An earthquake is considered as one of the most destructive natural disasters [86]. Buildings have partially or totally collapsed during earthquakes [87] such as the ones in Valparaiso, Chile in 1985 [88]; Mexico City in 1985 [89],[90]; Luzon, Philippines in 1990 [91]; Guam in 1993 [92] ; Northridge, Calif. in 1994 [93]; Kocaeli, Turkey in 1999 [94]; Chi-Chi, Taiwan in 1999 [95]; and Bhuj, India in $2001[96]$.

Several of the structural collapses occurred in buildings that were designed with accordance to modern and current seismic design codes [87]. Some of the 
Table 8.1: Some of the Deadliest Earthquakes [97]

\begin{tabular}{|l|l|l|r|}
\hline Date & Location & Deaths & Magnitude \\
\hline July 27, 1976 & China, Tangshan & 655,000 (estimated) & 7.5 \\
June 20, 1990 & Iran & 50,000 & 7.7 \\
Dec. 26, 2004 & Sumatra, Indonesia & 227,898 & 9.1 \\
Oct. 8, 2005 & Pakistan & 85,000 & 7.6 \\
May 12, 2008 & Sichuan, China & 87,586 & 7.9 \\
Jan. 12, 2010 & Haiti & 222,570 & 7 \\
\hline
\end{tabular}

deadliest earthquakes are displayed in Table 8.1, where the magnitude, the number of deaths the location and year are shown. The data shows that there is a significantly huge death toll as a circumstance to those earthquakes. For this reason, building design codes take into account the effect of earthquakes so that the structures can resist the natural occurrence in case it was to happen.

\subsubsection{How do earthquakes occur?}

An earthquake is the shaking and vibration of the earth's crust due to movement of the earth's plates (plate tectonics) [98]. When tectonic plates do not move smoothly against one another, a pressure build up occurs. When the pressure is released, an earthquake occurs. The point at which the pressure is released is called the focus. The point on the Earth's surface above the focus is called the epicentre. The energy of the earthquake is released in seismic waves that spread out from the focus. The most severe damage occurs closest to the epicentre. These terms are better displayed in Figure 8.1.

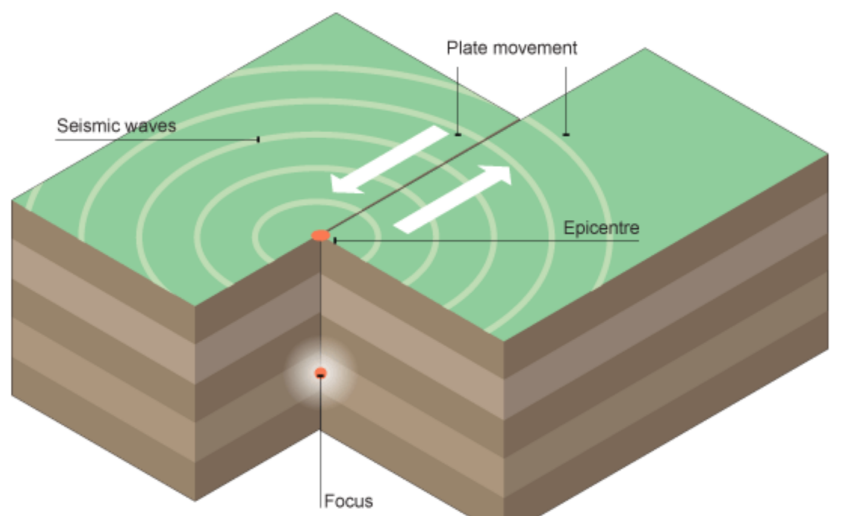

Figure 8.1: Focus, epicentre and seismic waves demonstration [99]

Understanding the earthquakes that may occur in regional areas is an impor- 
tant factor in structural design of buildings. Every area has its own "scale" based on previous occurrences of earthquakes in that region. Structural design codes attribute an earthquake load factor as well as a region code. Therefore, analysing whether a structure will fail under seismic loading is very important in design of structure.

\subsubsection{Seismic Scales}

Two common measures are used to characterise ground motion: acceleration, and intensity. The acceleration is usually measured by seismometers that work during intensive shaking near an earthquake. An example of the data obtained from a seismograph is shown in Figure 8.2. This data is from the Indonesia earthquake that occurred in 2004. Seismic hazards of areas estimate how probable an area is likely to experience a certain acceleration at a given time [100]. Intensity is a descriptive way of presenting earthquakes, and the Modified Mercalli intensity (MMI) scale is used, which uses Roman scales to present the intensity, where I is generally unfelt and XII is total destruction [100]. Table 8.2 shows the difference between the Mercalli and Richter scales in how they are measured, calculated and scaled. Table 8.3 lists the intensity of the scale and what each number could represent.

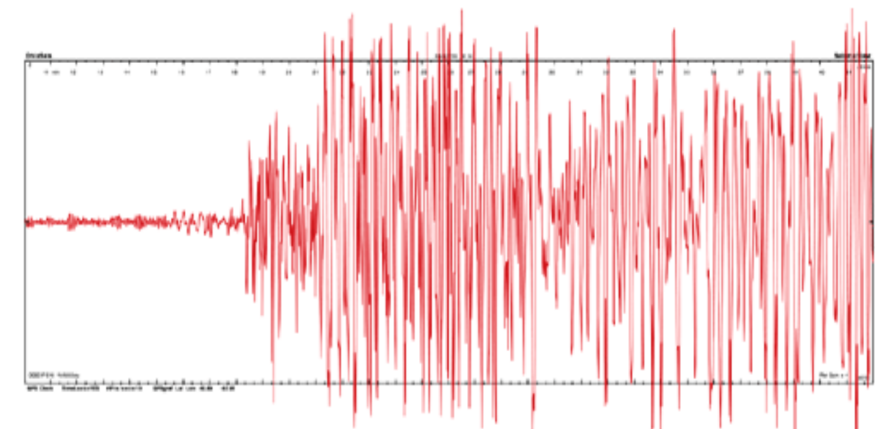

Figure 8.2: Seismogram of Sumatra, Indonesia 2004 earthquake [101]

\subsubsection{Structural Damage}

Figures 8.3b displays a building that collapsed in the Kobe, Japan 1995 earthquake. The death toll for that earthquake was 5,100 people [103]. The earthquake had a magnitude of 7.2, and a duration of 20 seconds. Since 1981, many of the 
Table 8.2: Methods of measuring earthquakes [102]

\begin{tabular}{|c|c|c|}
\hline & Mercalli Scale & Richter Scale \\
\hline Measures & Effects caused by earthquake & Energy released by earthquake \\
\hline $\begin{array}{l}\text { Measuring } \\
\text { Tool }\end{array}$ & Observation & Seismograph \\
\hline Calculation & $\begin{array}{l}\text { Quantified from observation of } \\
\text { effect on earths surface, hu- } \\
\text { man, objects and man-made } \\
\text { structures }\end{array}$ & $\begin{array}{l}\text { Base-10 logarithmic scale ob- } \\
\text { tained by calculating loga- } \\
\text { rithm of the amplitude of } \\
\text { waves. }\end{array}$ \\
\hline Scale & $\begin{array}{l}\text { I (not felt) to XII (total de- } \\
\text { struction) }\end{array}$ & $\begin{array}{l}\text { From } 2.0 \text { to } 10.0+. \\
3.0 \text { earthquake is } 10 \text { times } \\
\text { stronger than a } 2.0 \text { one. }\end{array}$ \\
\hline Consistency & $\begin{array}{l}\text { Varies depending on distance } \\
\text { from epicentre }\end{array}$ & $\begin{array}{l}\text { Varies at different distances } \\
\text { from the epicentre, but one } \\
\text { value is given for the earth- } \\
\text { quake as a whole. }\end{array}$ \\
\hline
\end{tabular}

structures built had been designed to strict seismic codes. Newly built high rise structures were generally undamaged. Figure 8.3a shows a woman walking in Kobe the day after the earthquake. Figure 8.3b shows a satellite image of Sumatra Indonesia in 2004 before and after the earthquake with magnitude 9.1 took place.

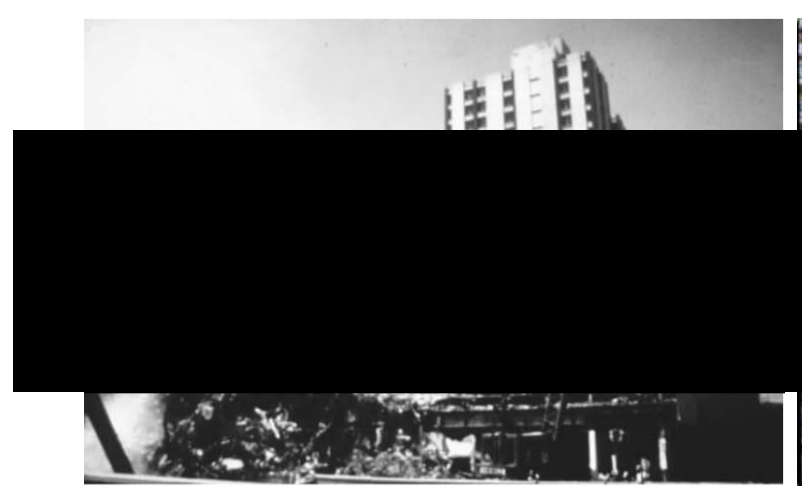

(a) Structural collapse during the 1985 Mexico City earthquake
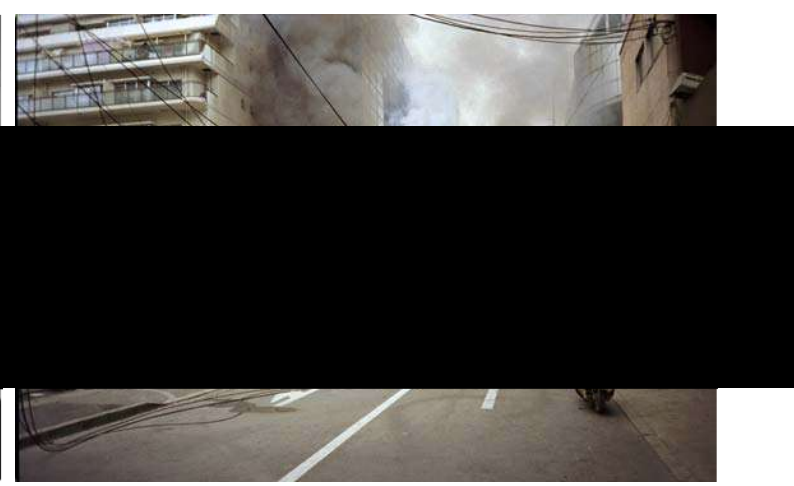

(b) Kobe, Japan 1995 earthquake [104] 
Table 8.3: Mercalli scale \& Richter scale [102]

\begin{tabular}{l|l|l}
\hline Mercalli & Richter & Witness Observations \\
\hline I & 1.0 to 2.0 & Felt by very few people; barely noticeable. \\
\hline II & 2.0 to 3.0 & Felt by a few people, especially on upper floors. \\
\hline III & 3.0 to 4.0 & $\begin{array}{l}\text { Noticeable indoors, especially on upper floors, but } \\
\text { may not be recognized as an earthquake. }\end{array}$ \\
\hline IV & 4 & $\begin{array}{l}\text { Felt by many indoors, few outdoors. May feel like } \\
\text { heavy truck passing by. }\end{array}$ \\
\hline V & VI 5.0 & $\begin{array}{l}\text { Felt by almost everyone, some people awakened. } \\
\text { Small objects moved. Trees and poles may shake. }\end{array}$ \\
\hline VII & 5.0 to 6.0 & $\begin{array}{l}\text { Felt by everyone. Difficult to stand. Some heavy } \\
\text { furniture moved, some plaster falls. Chimneys may } \\
\text { be slightly damaged. }\end{array}$ \\
\hline VIII & 6.0 to 7.0 & $\begin{array}{l}\text { Slight to moderate damage in well built, ordinary } \\
\text { structures. Considerable damage to poorly built } \\
\text { structures. Some walls may fall. }\end{array}$ \\
\hline IX & $\begin{array}{l}\text { Little damage in specially built structures. Consid- } \\
\text { erable damage to ordinary buildings, severe damage } \\
\text { to poorly built structures. Some walls collapse. }\end{array}$ \\
\hline XII & 7.0 to 8.0 & $\begin{array}{l}\text { Considerable damage to specially built structures, } \\
\text { buildings shifted off foundations. Ground cracked } \\
\text { noticeably. Wholesale destruction. Landslides. }\end{array}$ \\
\hline & $\begin{array}{l}\text { Most masonry and frame structures and their foun- } \\
\text { dations destroyed. Ground badly cracked. Land- } \\
\text { slides. Wholesale destruction. }\end{array}$ \\
\hline or & $\begin{array}{l}\text { Total damage. Few, if any, structures standing. } \\
\text { Bridges destroyed. Wide cracks in ground. Waves } \\
\text { seen on ground. }\end{array}$ \\
\hline $\begin{array}{l}\text { Total damage. Waves seen on ground. Objects } \\
\text { thrown up into air. }\end{array}$ \\
\hline
\end{tabular}




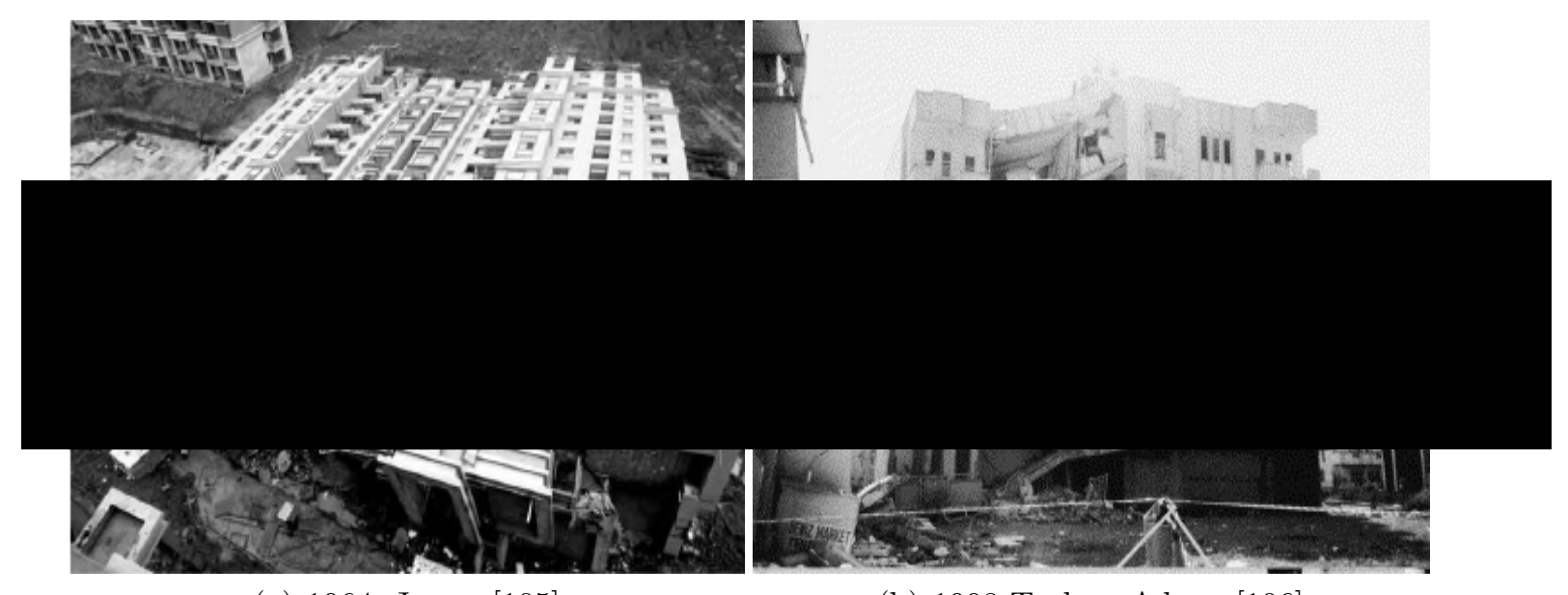

(a) 1964, Japan [105]

(b) 1998 Turkey, Adana [106]

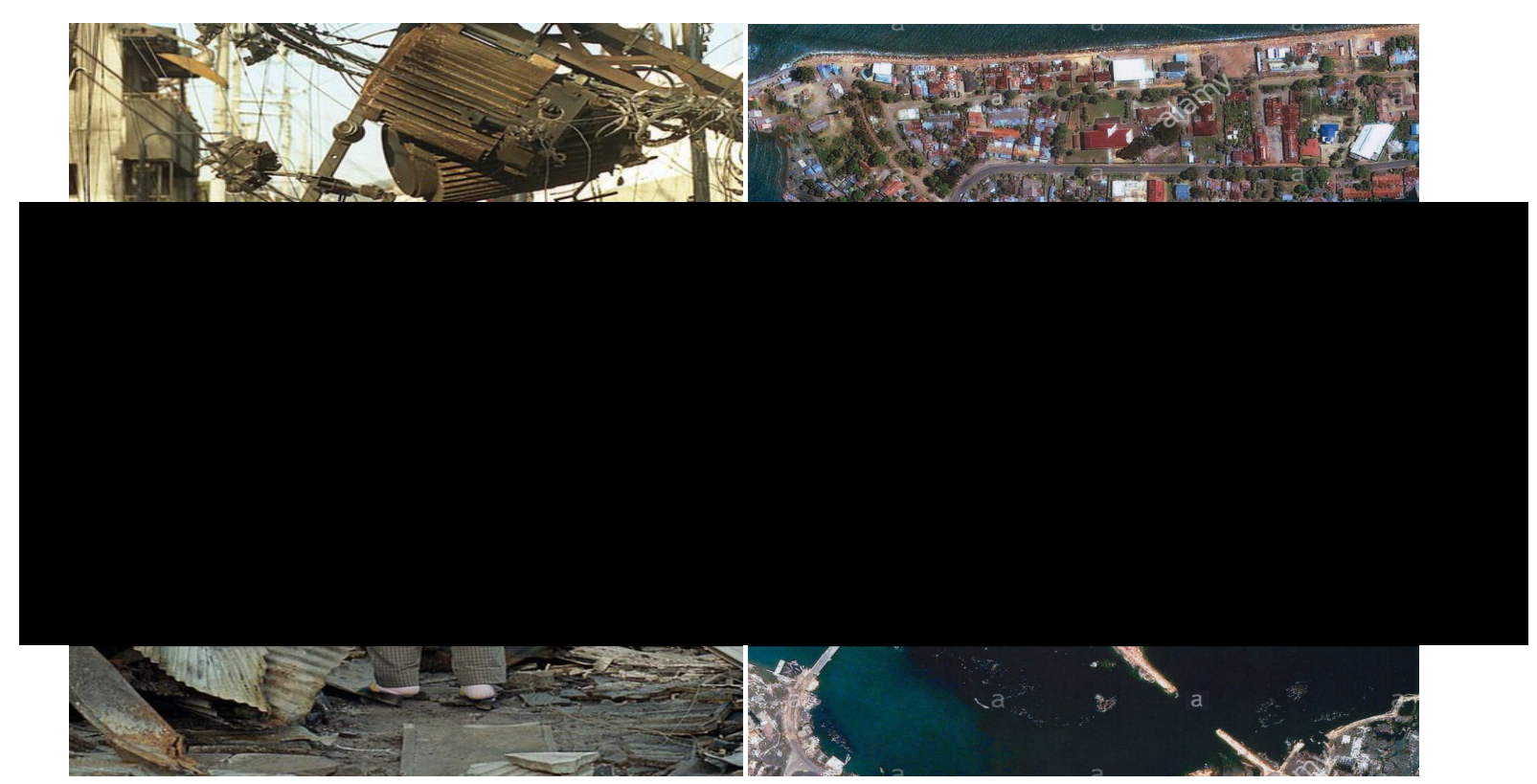

(a) Woman walking in Kobe the day after the (b) A satellite image of Sumatra, Indonesia, earthquake [107] before and after [108]

\subsection{Earthquake Implementation in AEM}

Although the damage to structures from earthquakes are quite severe, modelling the effect of a past earthquake on a linear elastic structure is straightforward. Recalling the equation of motion, there will be an extra external load applied to the structure which represents the earthquake effect. The data needed is the acceleration of the ground motion. The force equation due to the earthquake is shown in Equation 8.1.

$$
F_{Q}(t)=-M \mathbf{d}\left(\ddot{u}_{Q}(t)\right)
$$


where $F_{Q}(t)$ is the earthquake force vector at time step $t, M$ is the diagonal mass matrix, $\mathbf{d}$ is the earthquake input vector, and $\ddot{u}_{Q}(t)$ is the ground acceleration of the earthquake at time step $t$. To represent ground motion in the x-direction, the $\mathrm{x}$-degrees of freedom in the vector contain the value of the acceleration. It is similar for the y-degrees of freedom if there was ground motion in the y-direction. The gravity effect is also included to the equation of motion by Equation 8.2.

$$
F_{g}=M\left(\ddot{u}_{g}\right)
$$

where, $F_{g}$ is the gravity force vector, and $\ddot{u}_{g}$ is $-9.81 \mathrm{~m} / \mathrm{s}^{2}$ at every y degreeof-freedom. The equation of motion at every time step is therefore shown as Equation 8.3 for a nonlinear case. Representing a linear dynamic case is having $F_{\text {int }}=K u$.

$$
M \ddot{u}+C \dot{u}+F_{\text {int }}=F_{\text {ext }}(t)+F_{g}+F_{Q}(t)
$$

\subsubsection{Earthquake Data}

The acceleration used for the earthquake load applied to the following examples is based on the $1940 \mathrm{El}$ Centro Earthquake. The acceleration time-history plot is shown in Figure 8.3. The time history for the Kobe 1995 earthquake is shown in Figure 8.4. The El Centro data was recorded for 50 seconds, while the Kobe data was recorded for 140 seconds. From the two graphs it can be seen that the Kobe earthquake was more powerful since it had a maximum acceleration of almost $8 \times 10^{4} \mathrm{~m} / \mathrm{sec}$ while, the El Centro earthquake had a maximum acceleration of $3.5 \times 10^{4} \mathrm{~m} / \mathrm{sec}$. The data is collected from reference [109]. There are several more

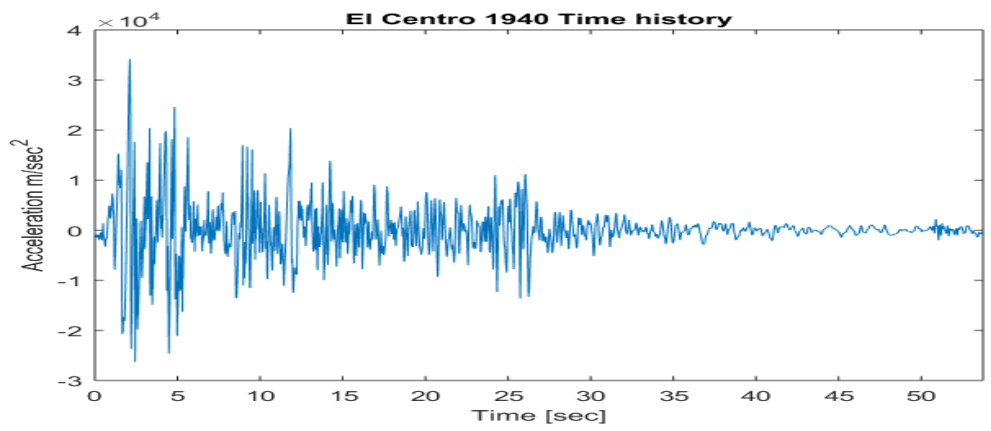

Figure 8.3: El Centro 1940 time history acceleration graph 


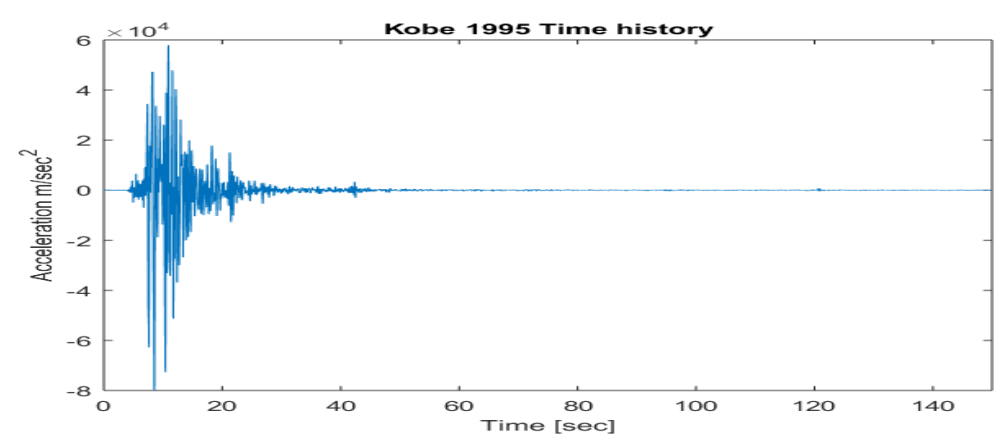

Figure 8.4: Kobe, Japan 1995 time history acceleration graph

earthquake data that are available, however for the purpose of demonstration only the El Centro and Kobe earthquakes are considered. In the following sections the implementation of earthquake data to the Applied Element code is presented for analysis of $2 \mathrm{D}$ frames.

\subsubsection{Frame Implementation}

2D frames with different loads, heights and floors are analysed using the Gaussian AEM in this section. As mentioned in previous chapters, the code is written in MATLAB. The frame nodes and connectivities are generated in ANSYS Mechanical APDL. ANSYS exports a data file containing the number of nodes, the table of connectivities and the degrees of freedom. MATLAB is used to read the exported data files from ANSYS to create the frame and begin the analysis of the frame using the Gaussian springs based AEM.

\subsection{Applications}

Some of the applications of the frame analysis to model the progressive collapse behaviour of structures are presented in this section. Different frame dimensions are considered and subject to the Kobe earthquake. There is no contact considered between elements.

\subsubsection{1x1 Frame}

The frame shown in Figure 8.5 is analysed for the point load applied at the midspan of the roof level shown, as well as with base excitation due to earthquake 
loading, and inclusion of gravity effect. The properties of the materials are shown in Table 8.4. The ends at the boundary are fixed in the three degrees of freedom that are considered: horizontal direction, vertical direction and rotation. Figure 8.6 is the displacement, velocity and acceleration of the point at the midspan, before any failure in the frame occurs. Figure 8.7 is the displacement, velocity and acceleration. Figure 8.8 displays the deflection of the frame in the first millisecond. The deflection is factored so it can be seen. However, in reality the deflection cannot be seen and this is shown in the unscaled deflections presented in Figures 8.8a - 8.8c. Figure 8.9 presents the progressive collapse of the frame at different time steps until collapse occurs. As expected the frame first fails at the bottom right column near the boundary and later fails from the applied load that was at the midspan.

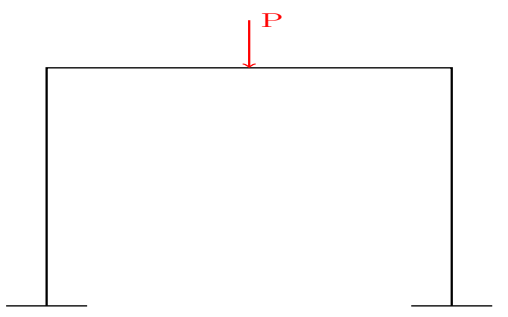

Figure 8.5: Frame with point load

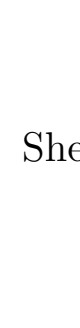

Modulus of Elasticity E Shear Modulus of Elasticity G Yield Stress

Beam span

Column span

cross-section width cross-section thickness

Applied Load
Value Units 200,000 [MPa] $76,923 \quad[\mathrm{MPa}]$ $250[\mathrm{MPa}]$ $3 \quad[\mathrm{~m}]$

$5 \quad[\mathrm{~m}]$

$0.15 \quad[\mathrm{~m}]$

$0.15[\mathrm{~m}]$ $1000 \quad[\mathrm{~N}]$

Table 8.4: Section Properties
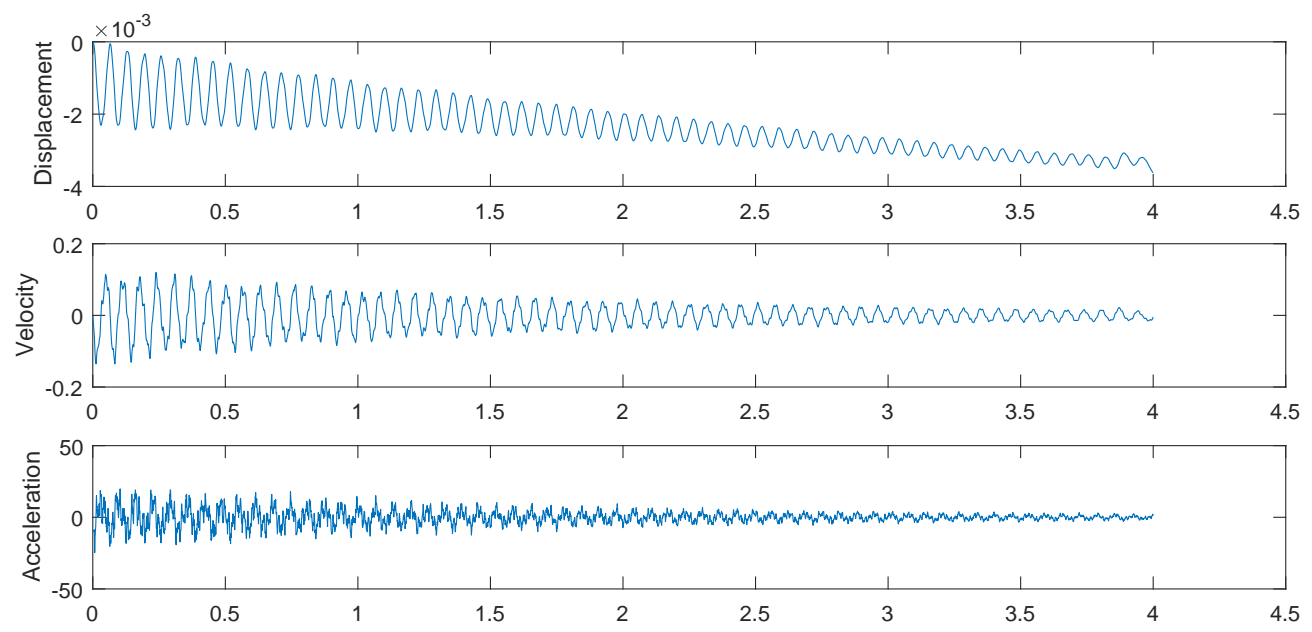

Figure 8.6: Acceleration, velocity and displacement vs. time at midspan before failure occurs 

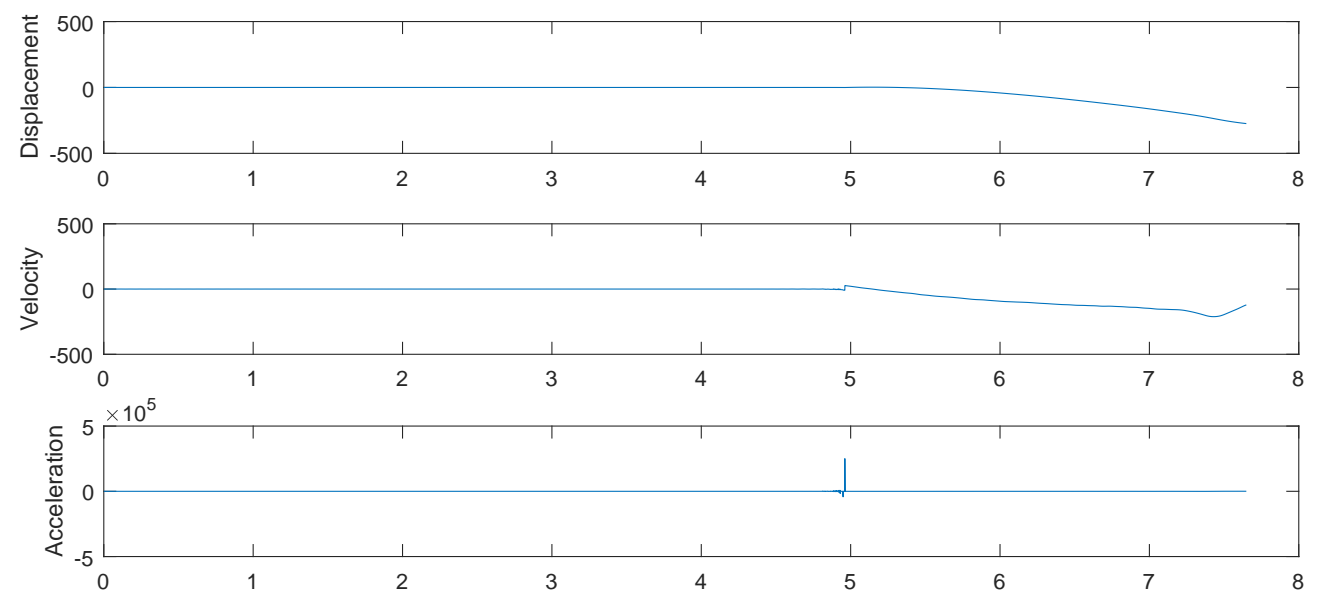

Figure 8.7: Displacement, velocity and acceleration after failure at midspan 


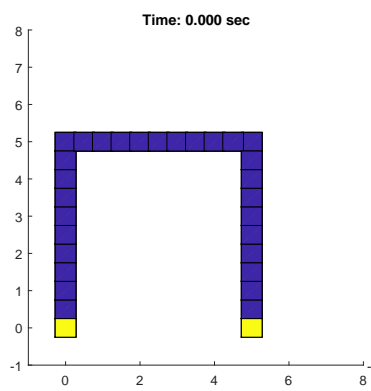

(a)

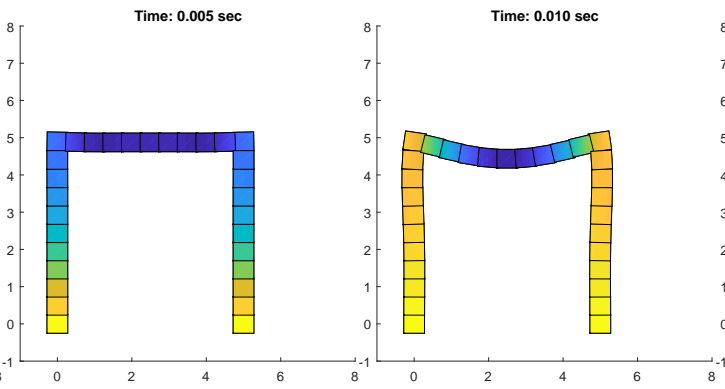

(b)

(c)

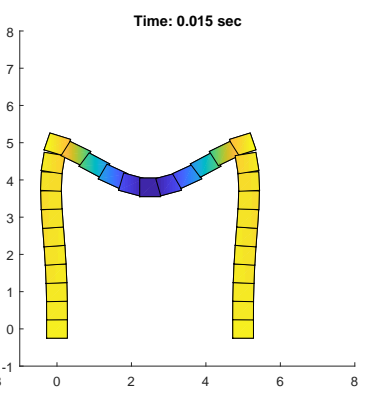

(d)

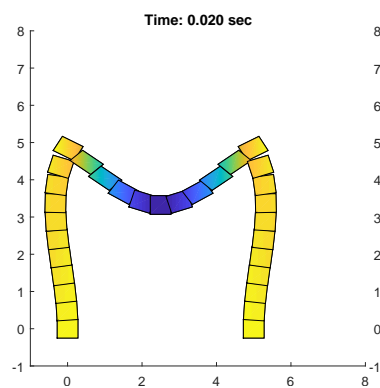

(e)

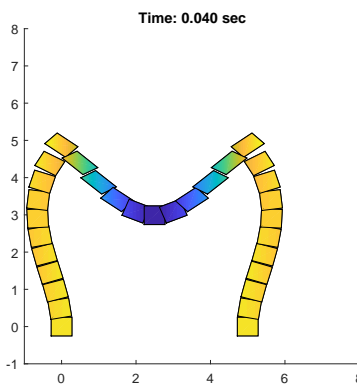

(i)

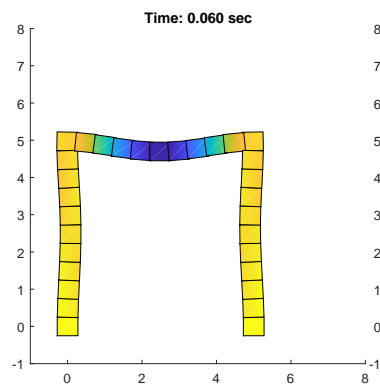

(m)

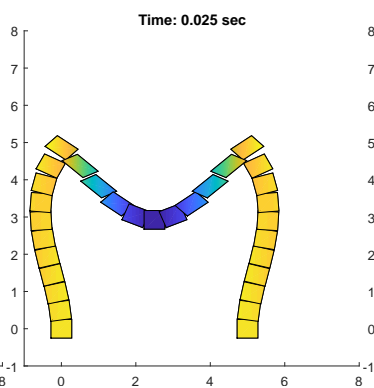

(f)

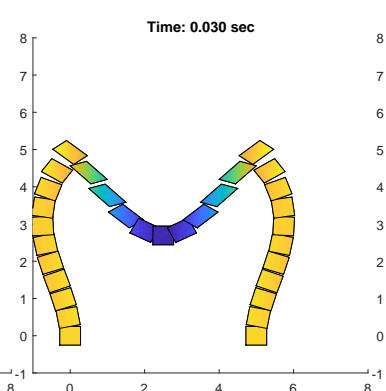

(g)

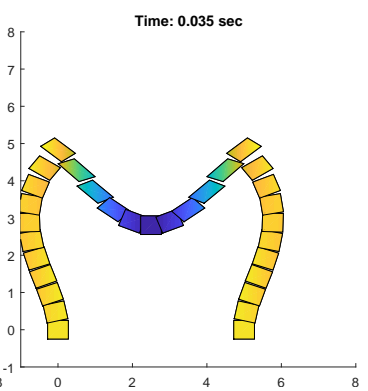

(h)

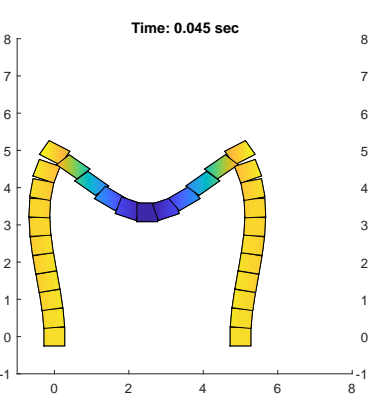

(j)

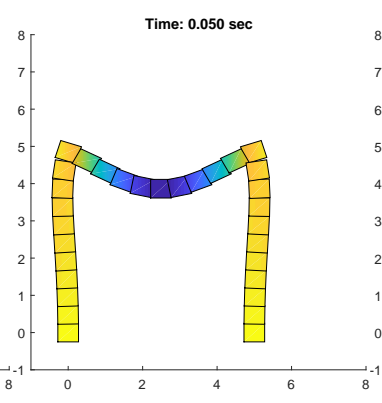

(k)

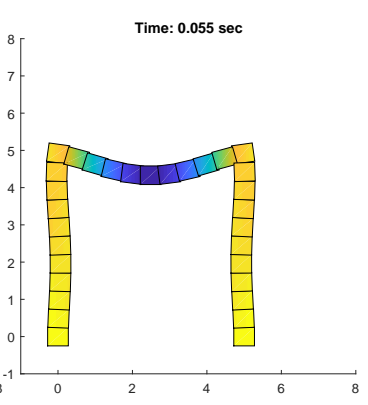

(l)

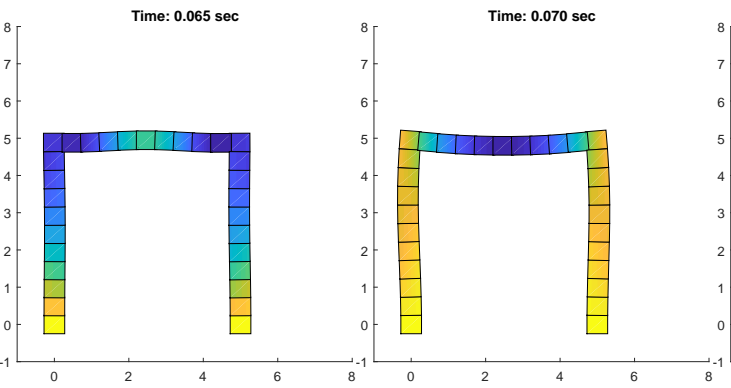

(n) (o)

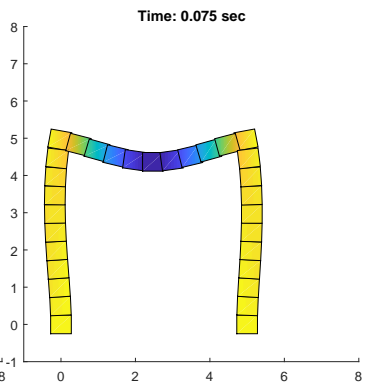

(p)

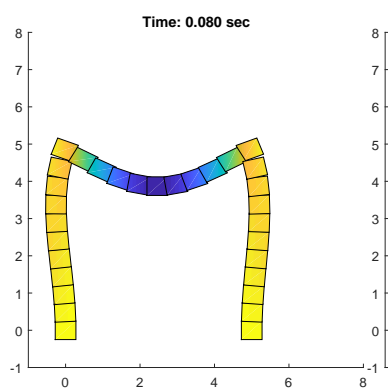

(q)

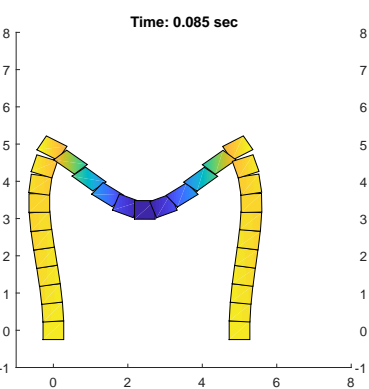

$(\mathrm{r})$

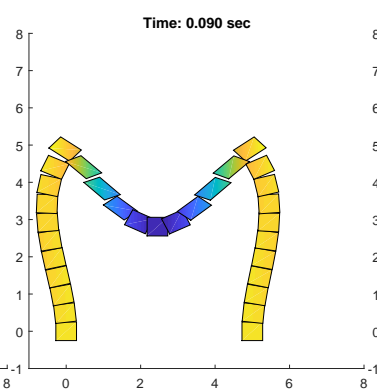

$(\mathrm{s})$

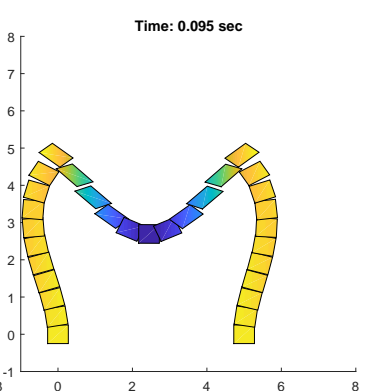

$(\mathrm{t})$

Figure 8.8: Factored frame deflection in first 0.1 second under earthquake Kobe load 


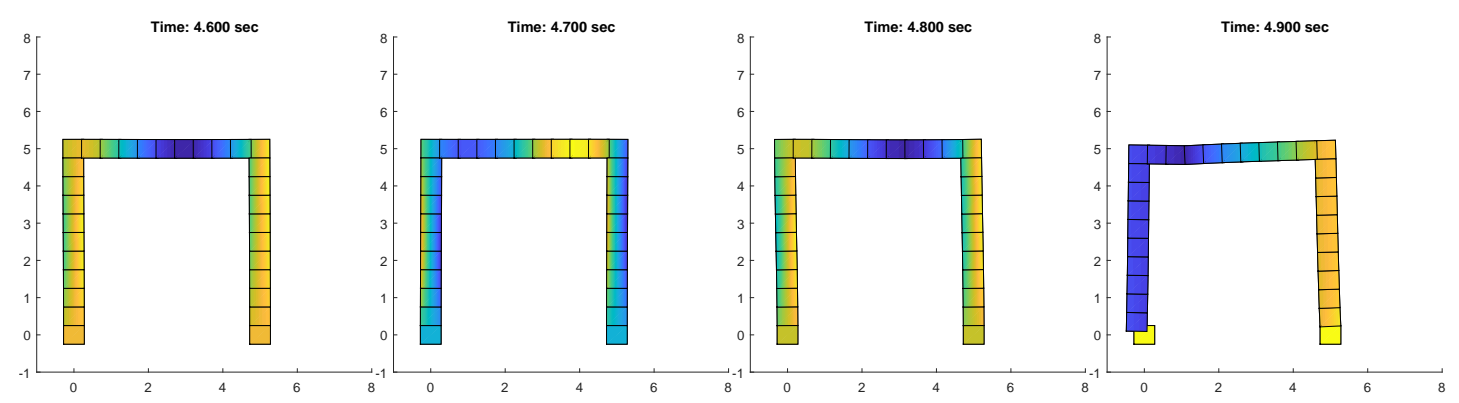
(a) $4.6 \mathrm{sec}$
(b) $4.7 \mathrm{sec}$
(c) $4.8 \mathrm{sec}$
(d) $4.9 \mathrm{sec}$

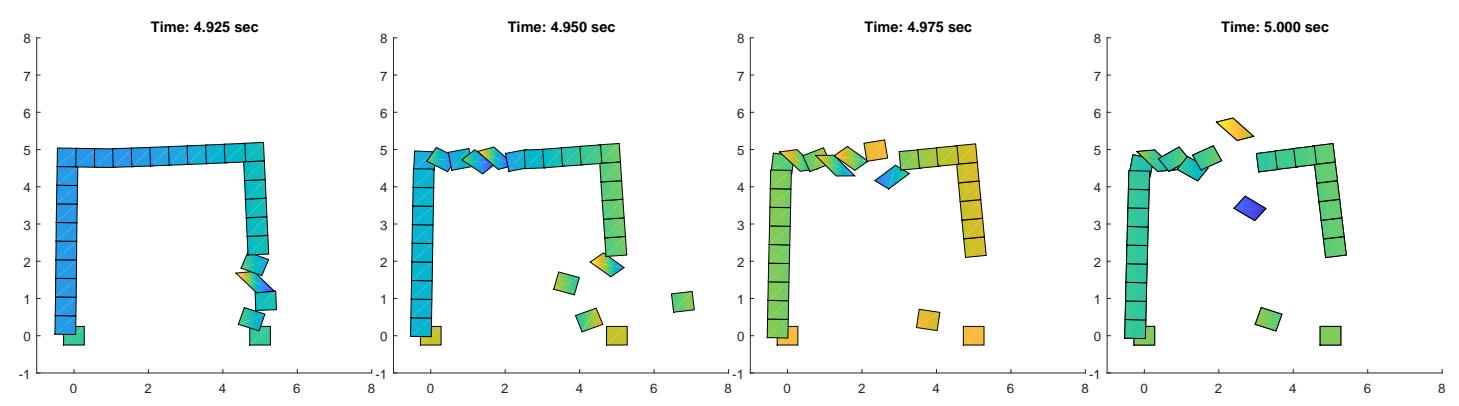

(e) $4.925 \mathrm{sec}$

(f) $4.95 \mathrm{sec}$

(g) $4.975 \mathrm{sec}$

(h) $5 \mathrm{sec}$
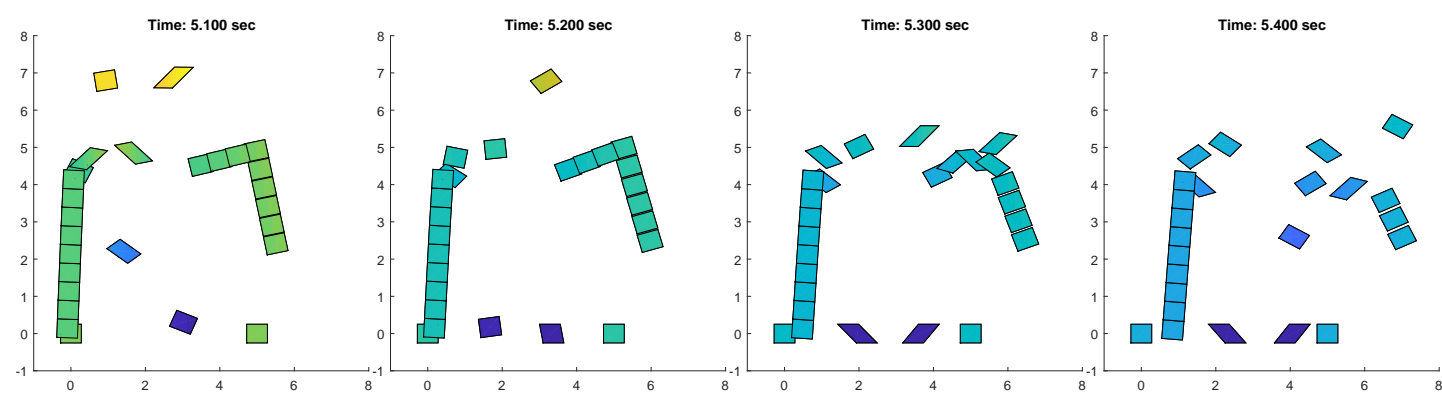

(i) $5.1 \mathrm{sec}$

(j) $5.2 \mathrm{sec}$

(k) $5.3 \mathrm{sec}$

(l) $5.4 \mathrm{sec}$

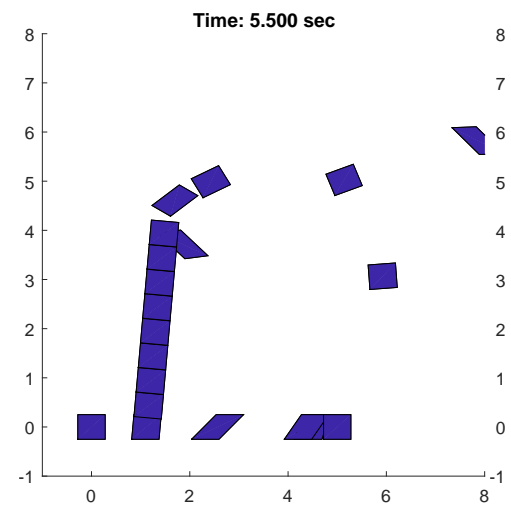

(m) $5.5 \mathrm{sec}$

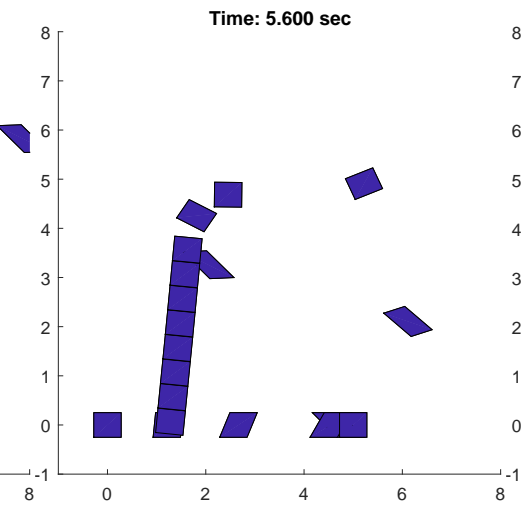

(n) $5.6 \mathrm{sec}$

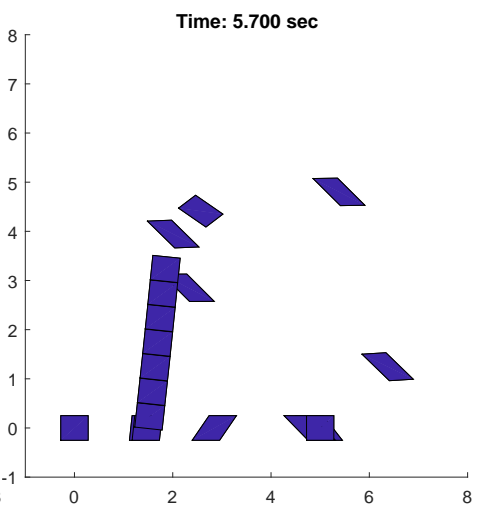

(o) $5.7 \mathrm{sec}$

Figure 8.9: Progressive collapse of $1 \mathrm{x} 1$ frame with earthquake Kobe loading and loading applied at midspan 


\subsubsection{3x8 Frame}

A frame with 8 floors and 3 bays is presented. The applied load on the frame is the El Centro earthquake, and a fixed incremental load applied in the positive $\mathrm{x}$-direction along the first floor of the frame. Gravity is included. The results are shown in Figures 8.10 and 8.11. It can be seen that the structure fails due to the external load first, and the remainder of the building progressively collapses.

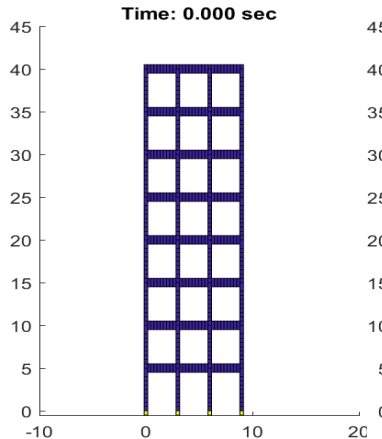

(a) $0 \mathrm{sec}$

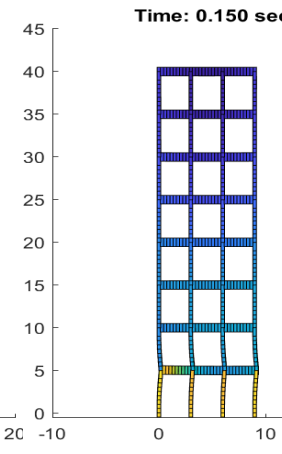

(b) $0.15 \mathrm{sec}$

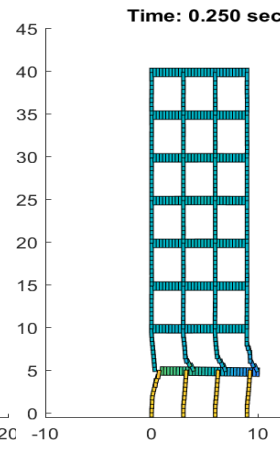

(c) $0.25 \mathrm{sec}$

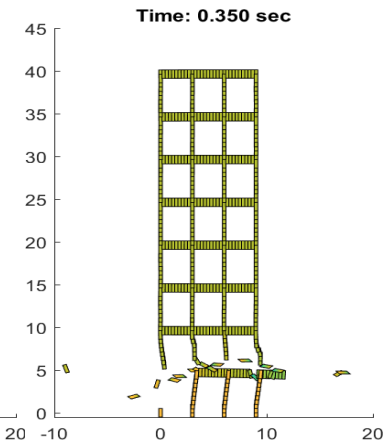

(d) $0.35 \mathrm{sec}$
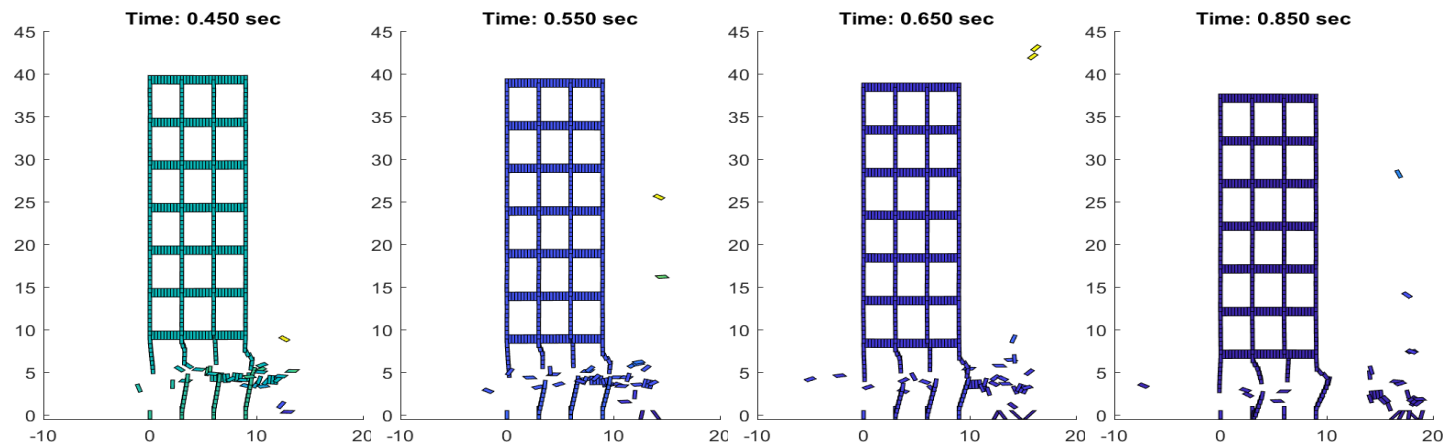

(e) $0.45 \mathrm{sec}$

(f) $0.55 \mathrm{sec}$

(g) $0.65 \mathrm{sec}$

(h) $0.85 \mathrm{sec}$
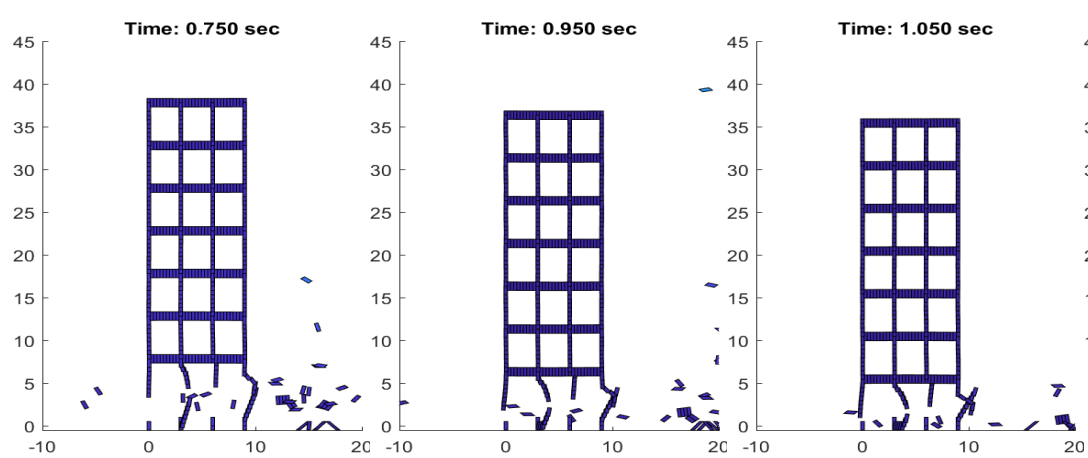

(i) $0.95 \mathrm{sec}$

(j) $0.95 \mathrm{sec}$

(k) $1.05 \mathrm{sec}$

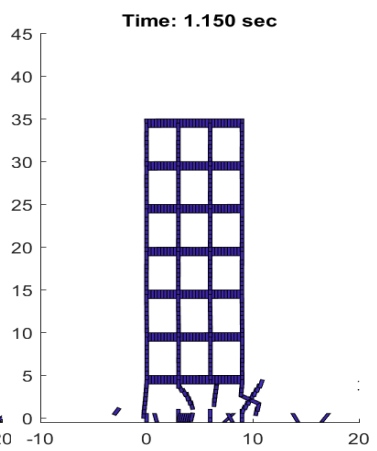

(l) $1.15 \mathrm{sec}$

Figure 8.10: Progressive collapse of 3x8 frame with earthquake loading and loading applied at midspan 


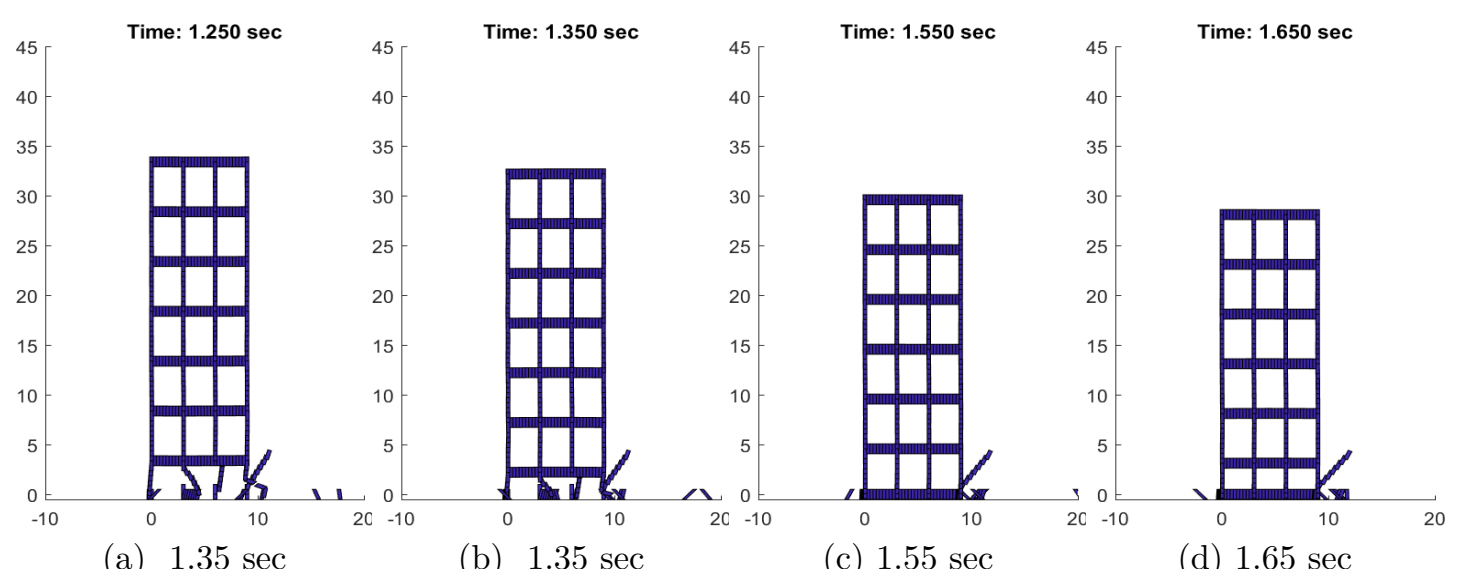

(a) $1.35 \mathrm{sec}$

(b) $1.35 \mathrm{sec}$

(c) $1.55 \mathrm{sec}$

(d) $1.65 \mathrm{sec}$
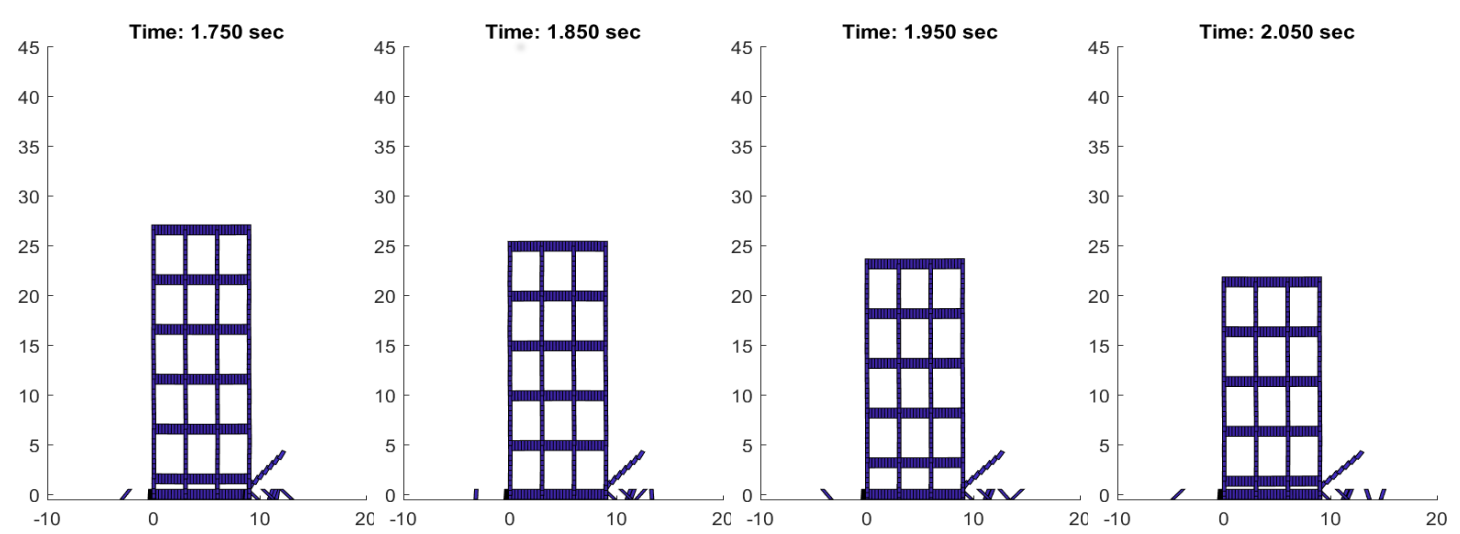

(e) $1.75 \mathrm{sec}$

(f) $1.85 \mathrm{sec}$

(g) $2.05 \mathrm{sec}$

(h) $2.05 \mathrm{sec}$
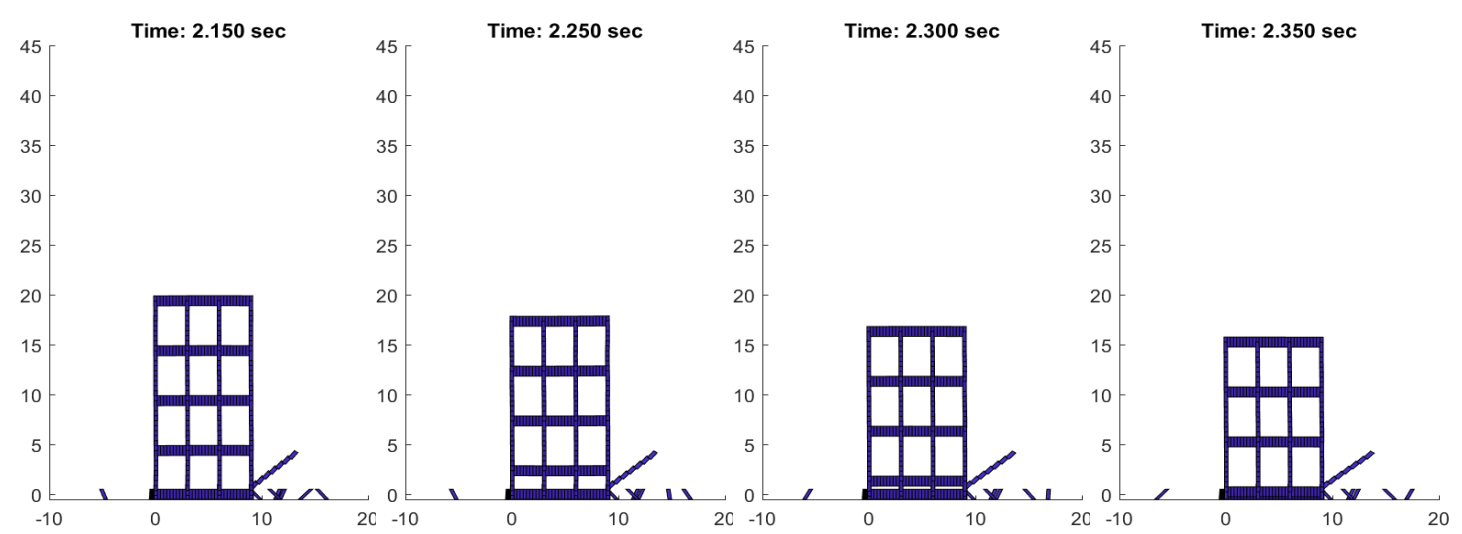

(i) $2.25 \mathrm{sec}$

(j) $2.25 \mathrm{sec}$

(k) $2.3 \mathrm{sec}$

(1) $2.35 \mathrm{sec}$

Figure 8.11: Progressive collapse of $3 \times 8$ frame with earthquake loading and loading applied at midspan (continued) 


\subsubsection{Multi-storey frame}

The next application is a multi-storey frame, shown in Figure 8.12, subject to the El Centro earthquake. The failure begins at the bottom left frame shown in Figure $8.12 \mathrm{c}$ at 0.85 seconds.

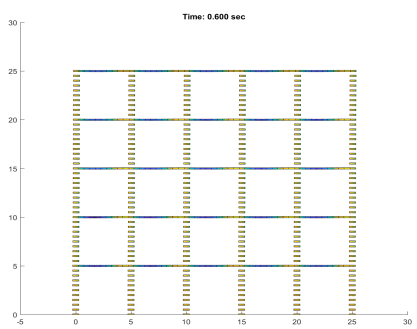

(a) $0.6 \mathrm{sec}$

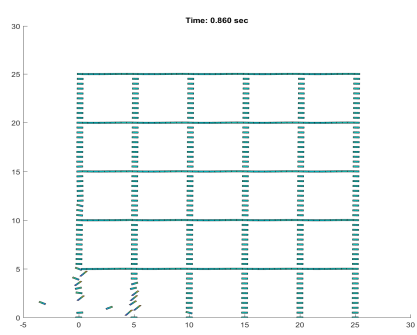

(d) $0.86 \mathrm{sec}$

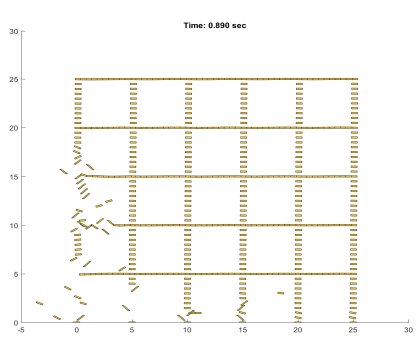

(g) $0.89 \mathrm{sec}$

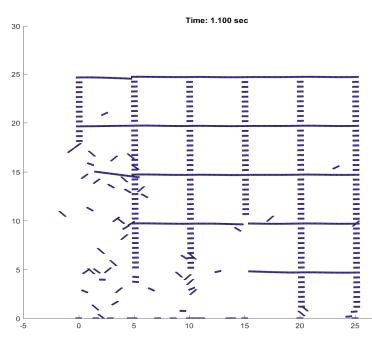

(j) $1.1 \mathrm{sec}$

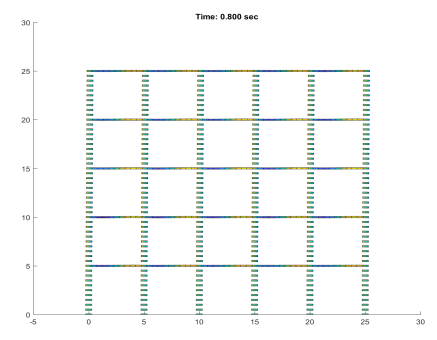

(b) $0.8 \mathrm{sec}$

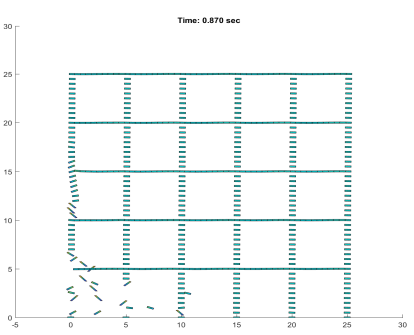

(e) $0.87 \mathrm{sec}$

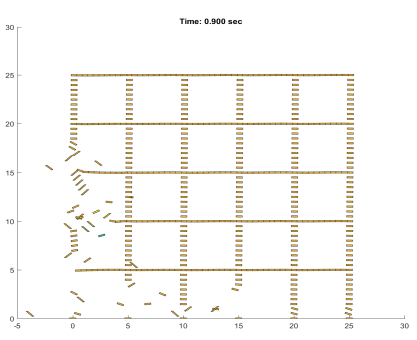

(h) $0.9 \mathrm{sec}$

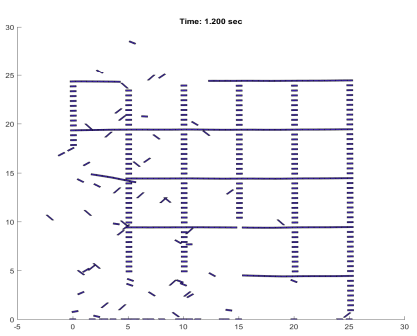

(k) $1.2 \mathrm{sec}$

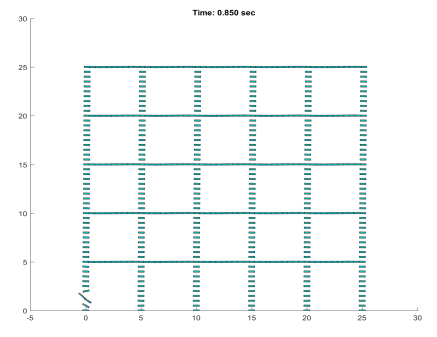

(c) $0.85 \mathrm{sec}$

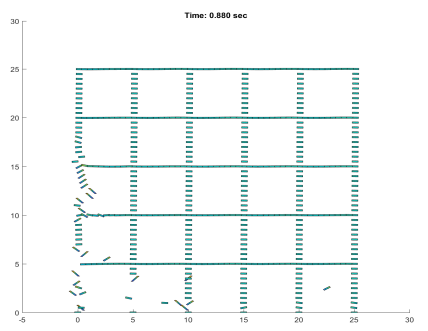

(f) $0.88 \mathrm{sec}$

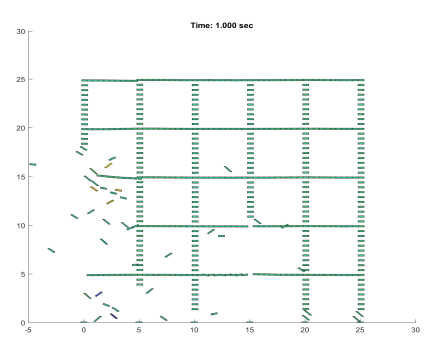

(i) $1 \mathrm{sec}$

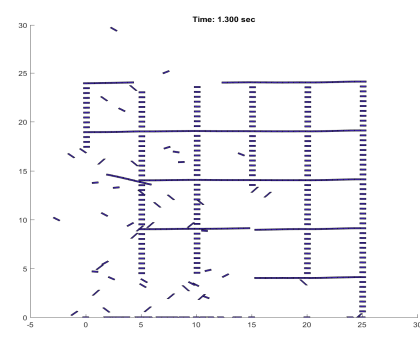

(1) $1.3 \mathrm{sec}$

Figure 8.12: Analysis of multi-storey steel frame subject to El Centro Earthquake 


\subsubsection{2x8 Frame}

Figure 8.13 depicts the progressive collapse of a $2 \times 8$ frame subject to the El Centro Earthquake as well as a point load in the positive y direction applied on the left wall at the first floor.

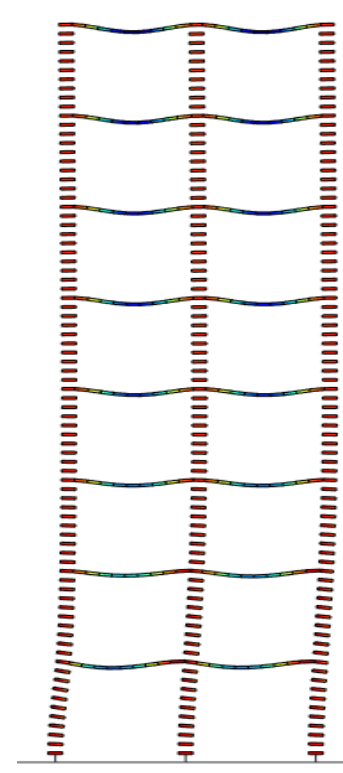

(a) $0.25 \mathrm{sec}$

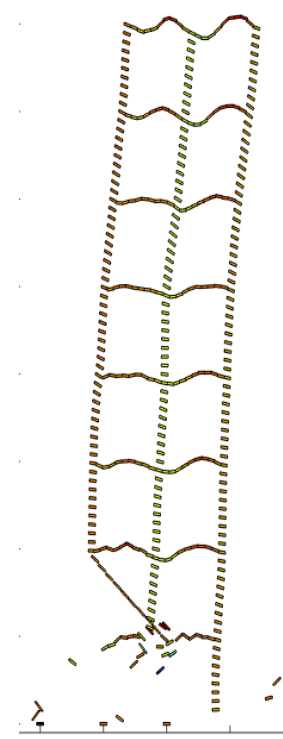

(e) $0.83 \mathrm{sec}$

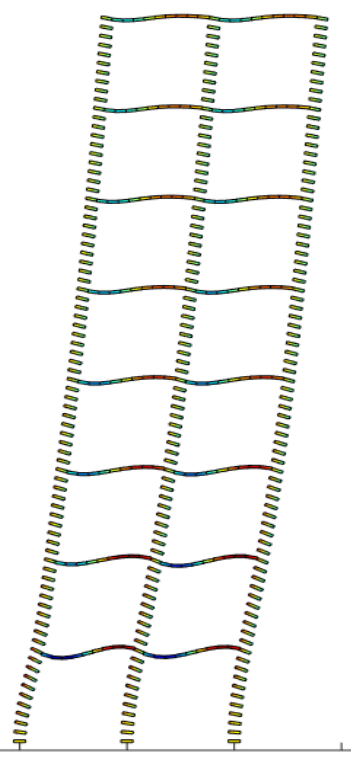

(b) $0.551 \mathrm{sec}$

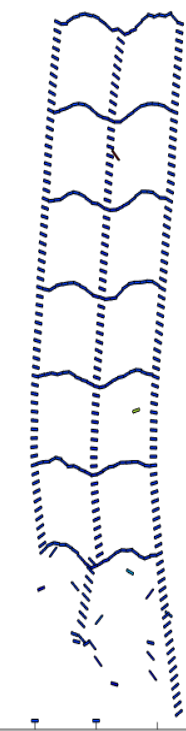

(f) $0.84 \mathrm{sec}$

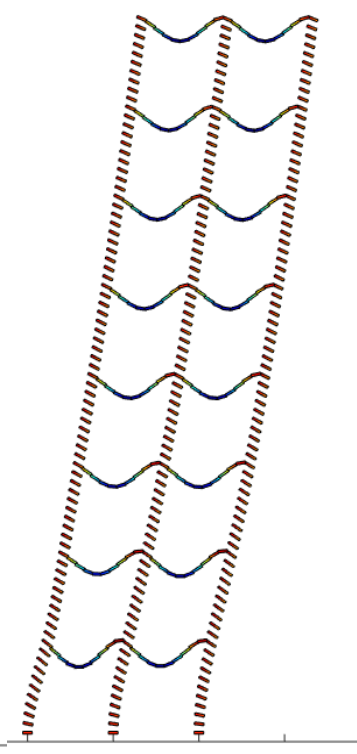

(c) $0.75 \mathrm{sec}$

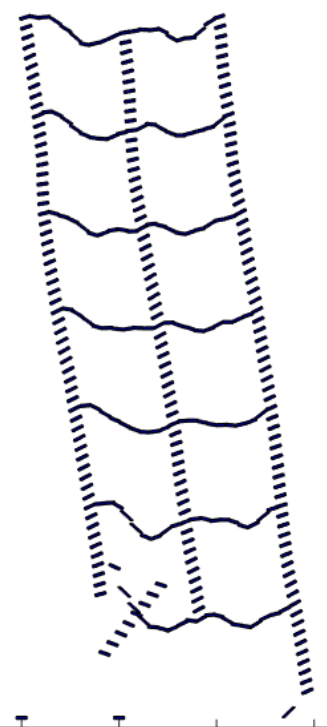

(g) $0.9 \mathrm{sec}$

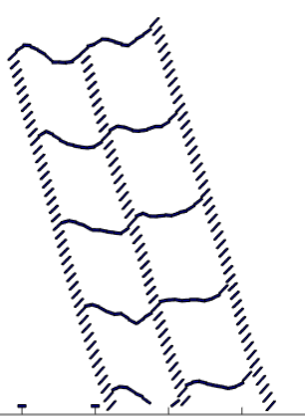

(h) $1 \mathrm{sec}$

Figure 8.13: Time history of a high rise frame undergoing seismic loading and point load at in positive $\mathrm{x}$-direction at first floor 


\subsubsection{High rise frame - 15 floors}

Figure 8.14 shows the progressive collapse behaviour of a frame with 15 floors. The figure does not show the total collapse of the frame, rather just until where major elements of the frame have failed. The total collapse of the structure can be seen if the simulation is run for longer. From the collapse it can be seen that first the structure undergo serious oscillations and finally collapses near the boundary.

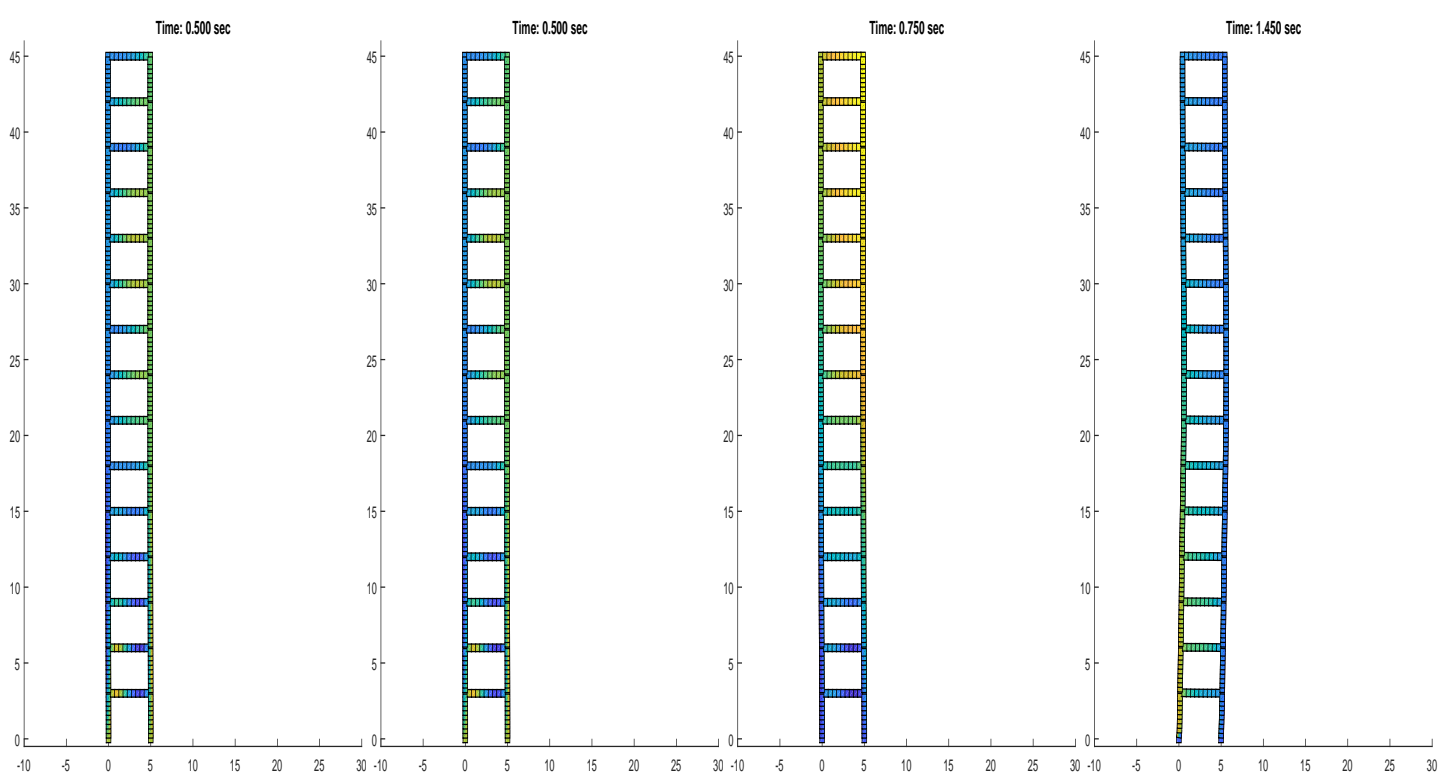

(a) $\mathrm{t}=0.25$

(b) $\mathrm{t}=0.551$

(c) $\mathrm{t}=0.75$

(d) $t=0.82$
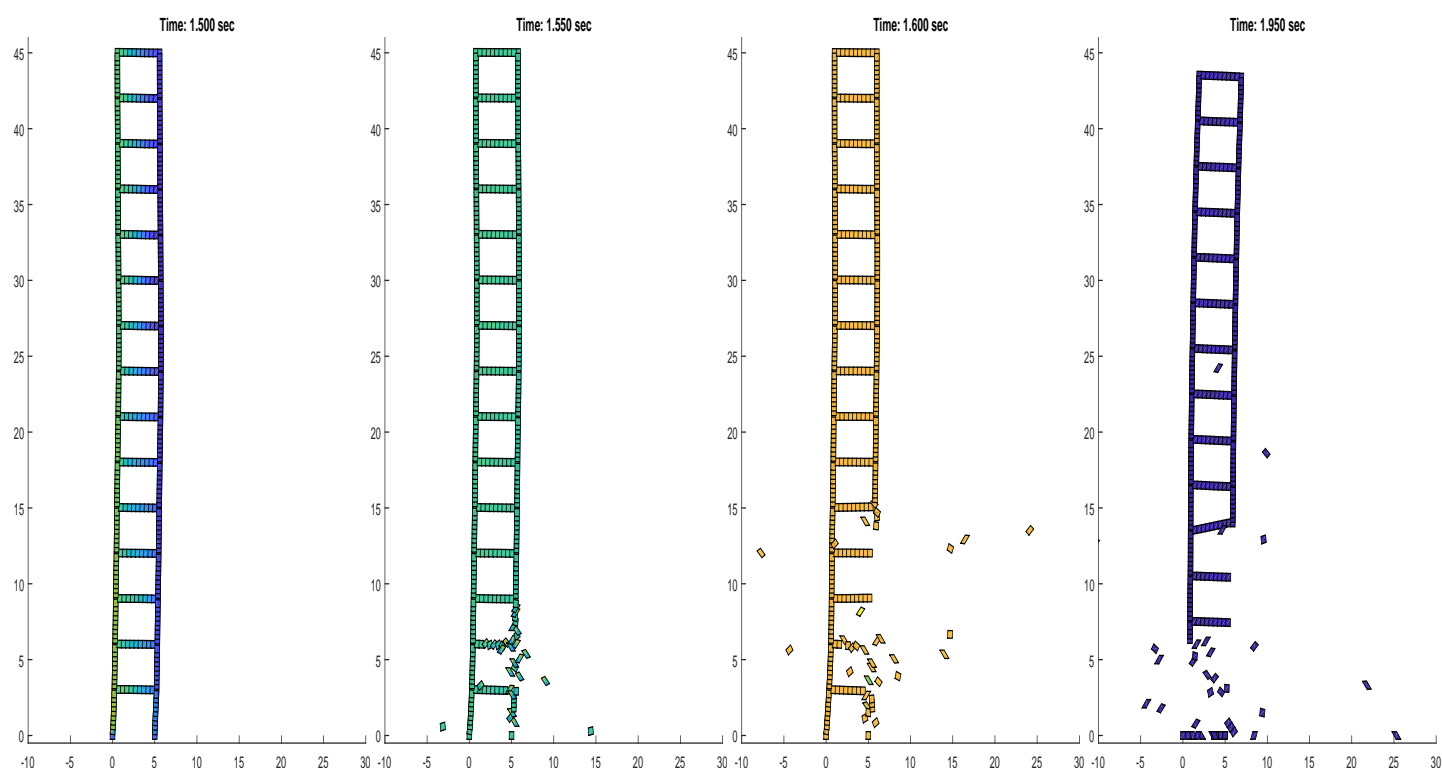

(e) $\mathrm{t}=0.83$

(f) $t=0.84$

(g) $\mathrm{t}=0.9$

(h) $\mathrm{t}=1$

Figure 8.14: Time history of a high rise frame undergoing seismic loading and point load at in positive $\mathrm{x}$-direction at first floor 


\subsection{Conclusion}

The progressive collapse of different structures subject to earthquakes were successfully analysed in this chapter. The analysis was performed using the Gaussian springs based AEM for nonlinear dynamic behaviour. The implementation of earthquake loading to the structure was quite simple, given that the time history data from an earthquake is presented. From the acceleration at every time step, the force is calculated, and applied as an external force on all degrees of freedom of the structure.

Different materials can be used to model the frames as well as reinforced concrete. Although some of the figures presented did not show total collapse of the structure, this is just due to the run time of the analysis. Future improvements would entail including contact between elements, and contact between the elements and the ground. When contact between elements is included, effect of failing neighbouring structures can be analysed. 


\section{Chapter 9}

\section{Structures Subject to Extreme Wind Loads}

In this chapter, the Gaussian spring based AEM is used to analyse structures subject to different weather conditions such as mean wind speeds, 3 second gusts and extreme conditions. Firstly, the weather condition is analysed in FLUENT to simulate the forces around the structure resulting from the applied wind load. The forces generated are then input into the Matlab code where the Gaussian spring based AEM is used to model the behaviour of structures due to the applied wind loads, and if the structure will experience collapse or not.

A brief introduction to the collapse of structures that occurred in the past due to wind loads is presented. Then the CFD model is built and explained. And finally, the structure is analysed in the MATLAB code to check for possible collapse of the structure. The purpose of this analysis is to present an application of wind loads to the structure that can be quite realistic, due to the wind being analysed on FLUENT first, rather than being applied as constant external forces.

\subsection{Collapse of Structures due to Wind loads}

In structural design codes, wind is usually represented by a speed profile. Its features and effects depend on the velocity of the wind, the geometry of the building and the protection from surrounding obstacles [110]. In slender structures, wind is one of the main load actions to be considered [110]. The environment has a 
great influence on a building and accurate analysis of the wind conditions should be experimentally produced using wind tunnel tests [111]. Low structures also undergo significant influences from wind, depending on the geometry plan and and the locations of the structures columns, the wind can create torsional forces in the frame.

There has been several recorded structural damages due to wind storms in the last 50 years. This is better presented in Figure 9.1, which shows the number of natural disasters since the 1950's - 2004 [112]. As can be seen, a large percentage of structural damage is due to wind storms rather than floods or earthquakes. Typhoon Mirielle in September 1991 caused 6 billion dollars of damage. There was 8 billion dollars in damages from 10 typhoons in Japan 2004, 28 billion dollars in economic losses along with 2,541 deaths in August 2005, from Hurricane Katrina [113]. This is why it is important to analyse structural behaviour under extreme weather conditions.

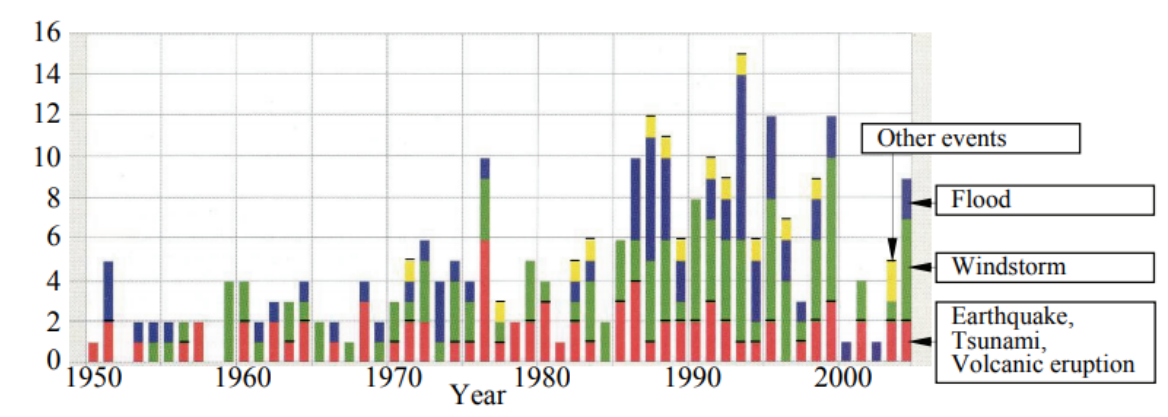

Figure 9.1: Devastating natural disasters. Red: Earthquake, Tsunami, Volcanic eruption. Blue: Flood. Green: wind-storm. Yellow: other events. [112]

Firstly a relationship between the mean wind speed and 3 second gust is explained. Table 9.1 displays the equivalence of different wind conditions with their corresponding damage associated. The damage does not only depend on the wind speed but also on the quality of the structure, so the table phenomena is not precise [113]. From the table it can be deduced that damage to structures starts to occur $40 \mathrm{~m} / \mathrm{s}$ gusts, and extreme damage starts with $45 \mathrm{~m} / \mathrm{s}$ mean speed, or $70 \mathrm{~m} / \mathrm{s}$ gusts. Therefore, these values will be used for analysis in the following sections. 
Table 9.1: Wind induced phenomena [113]

\begin{tabular}{|l|l|l|}
\multicolumn{2}{c|}{ Wind speed } & \multicolumn{1}{c|}{ Phenomena/Damage } \\
\hline $10 \mathrm{~min}$ mean & $3 \mathrm{~s}$ gust & \\
\hline $5 \mathrm{~m} / \mathrm{s}$ & $7-10 \mathrm{~m} / \mathrm{s}$ & - Vortex resonance/fatigue damage of truss members \\
\hline $10-15 \mathrm{~m} / \mathrm{s}$ & $15-20 \mathrm{~m} / \mathrm{s}$ & $\begin{array}{l}\text { - Handrail vibration/wind-induced noise } \\
\text { - Vortex resonance of steel chimneys } \\
\text { - Vibration perception in flexible high-rise buildings }\end{array}$ \\
\hline $20 \mathrm{~m} / \mathrm{s}$ & $30 \mathrm{~m} / \mathrm{s}$ & $\begin{array}{l}\text { - Seasickness and discomfort in high-rise buildings } \\
\text { - Damage to garage shutters } \\
\text { - Falling down of pedestrians }\end{array}$ \\
\hline $25 \mathrm{~m} / \mathrm{s}$ & $40 \mathrm{~m} / \mathrm{s}$ & $\begin{array}{l}\text { - Damage to roof tiles } \\
\text { - Damage to window panes due to wind-borne debris }\end{array}$ \\
\hline $30 \mathrm{~m} / \mathrm{s}$ & $45 \mathrm{~m} / \mathrm{s}$ & $\begin{array}{l}\text { - Collapse of RC block fences } \\
\text { - Damage to steel sheet roofing } \\
\text { - Overall roof lift-off - Collapse of wooden houses } \\
\text { - Falling down of gravestones }\end{array}$ \\
\hline $35 \mathrm{~m} / \mathrm{s}$ & $50 \mathrm{~m} / \mathrm{s}$ & $\begin{array}{l}\text { - Damage to window panes due to wind pressure } \\
\text { of high-rise buildings } \\
\text { - Blow over of heavy tombstones }\end{array}$ \\
\hline $40 \mathrm{~m} / \mathrm{s}$ & $60 \mathrm{~m} / \mathrm{s}$ & $\begin{array}{l}\text { - Damage to cladding of high-rise buildings } \\
\text { - Limit of allowable distortion of external } \\
\text { sealing compounds }\end{array}$ \\
\hline $45 \mathrm{~m} / \mathrm{s}$ & $70 \mathrm{~m} / \mathrm{s}$ & $\begin{array}{l}\text { - Main frame stresses in high-rise buildings } \\
\text { exceed elastic Limit }\end{array}$ \\
\hline
\end{tabular}




\section{Damage to structures}

Damage to structures due to recent typhoons are presented in this section. Figure 9.2 shows the damage to a soccer stadium in Korea, 2002. Figure 9.3 displays the damage to wind energy facilities due to Typhoon Maemi in 2003.

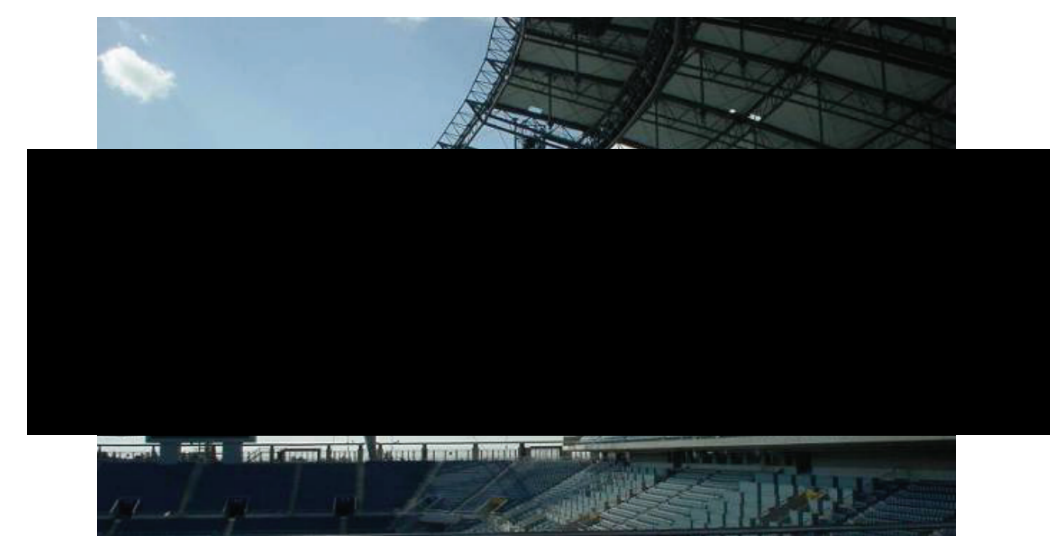

Figure 9.2: Damage to soccer stadium in Korea due to Typhoon Rusa, 2002 [113]

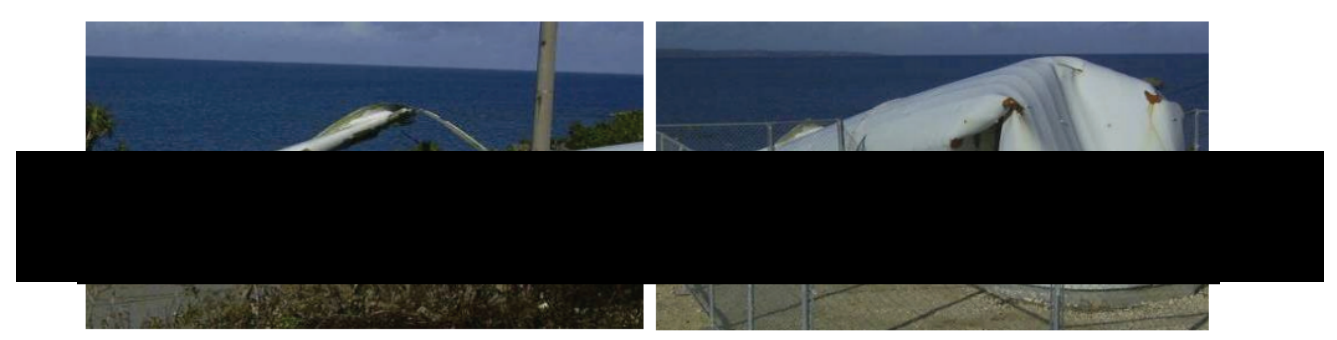

Figure 9.3: Damage to wind energy facilities in Miyakojima island due to Typhoon Maemi, 2003 [114]

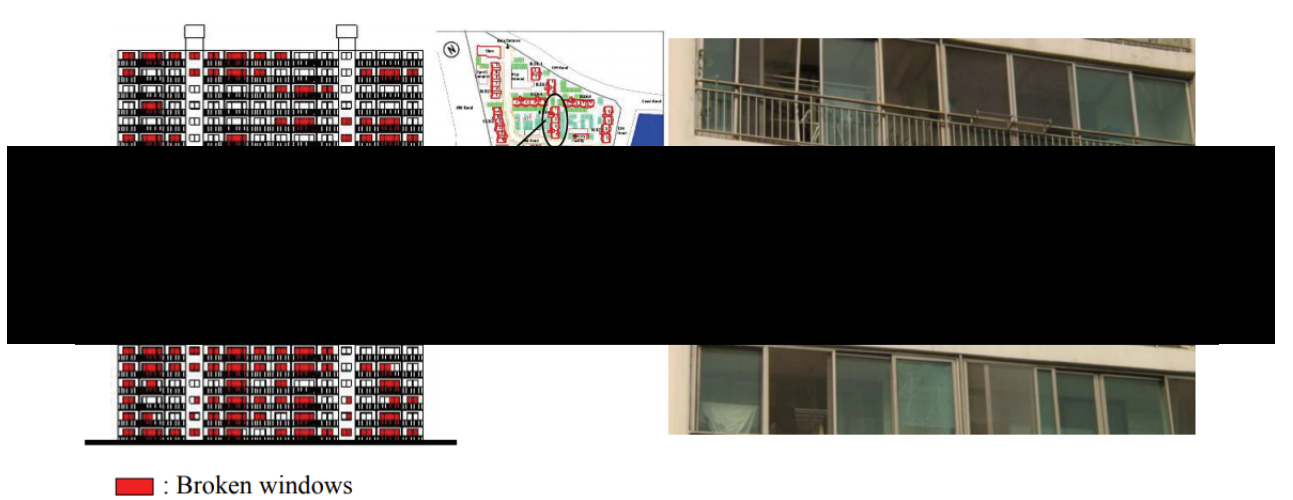

Figure 9.4: Broken windows in residential building in Korea, due to Typhoon Maemi [113] 


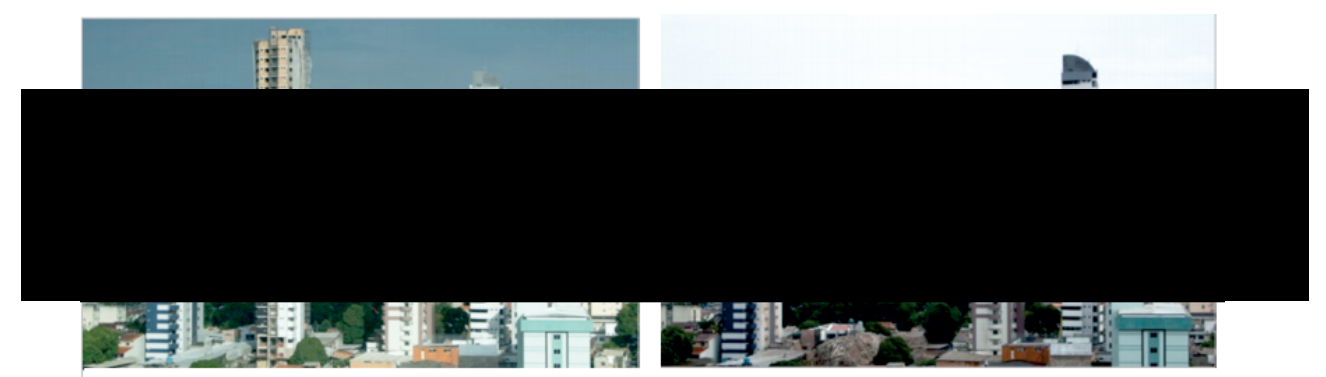

Figure 9.5: Collapse of building under construction undergoing intense winds [115]

\subsection{Using CFD for turbulent wind analysis}

Using computational fluid dynamics (CFD) for wind analysis is a fast and efficient way to determine the wind behaviour without needing to conduct expensive wind tunnel tests. There are some limitations on wind tunnel tests, such as tools scarcity, since big tunnels are required for urban models investigations and the scarcity in the number of measurement points in models [116]. The advantage of using CFD over wind tunnel tests is that the simulation gives a quantitative and qualitative wind flow representation of the whole volume rather than specific measurement points.

Several validation processes have been developed to improve and verify the accuracy of the CFD models in comparison to the wind tunnel tests. The FLUENT software is used for the CFD simulations [116].

In this project, FLUENT is used to model the wind behaviour and the forces exerted on the structure. The gust wind is modelled in FLUENT for 3 seconds. A general model and set up for the CFD model is presented and later the wind speeds will be changes to simulate the different conditions. From FLUENT we can obtain the force on each wall at every iteration. This is a kind of mean average force.

To implement the wind load in the AEM Matlab code, the force on each wall at each time step is applied on all the wall nodes. So the force obtained from the first time step is just one value for the whole wall. This value is applied to every node in that wall, and similarly for the roof, etc. At every next time step a new force is applied and the value is obtained from Ansys. This will be better explained with an example. 
Figure 9.6 shows an overview of how the processes will work. The geometry can be created in Ansys mechanical, and in parallel the forces due to the wind loads is obtained from FLUENT. Then the data files are exported to be read in the Matlab code for the frame to be analysed.

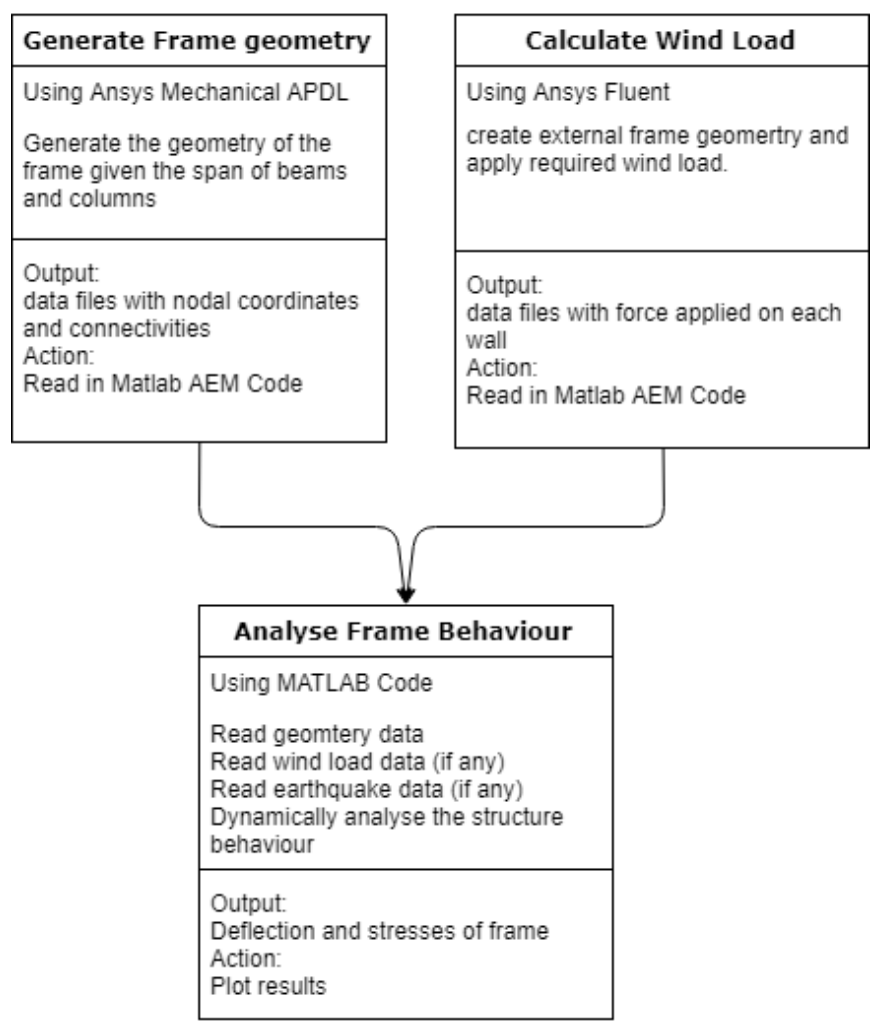

Figure 9.6: Flow chart for procedure

\subsubsection{Solution Model}

There are two major groups of turbulence models: RANS models (Reynoldsaveraged Navier-Stokes models) and LES models (Large Eddy Simulation Models). Among the RANS models are the standard $k-\epsilon$ model and its variations, as well as the Reynolds Stress Model (RSM). However, it has been known that the $k-\epsilon$ model is not appropriate for modelling airflow around buildings [117]. The RSM methods give better results for the flow around buildings. On the other hand, LES are more appropriate for analysis of wind around buildings because the solve the largest eddy and simulate only the smallest eddies [116]. However, LES methods require immense computational power, very fine meshing and long calculation time. Most common CFD software do not offer full LES models in 
three dimensions.

Case study validation test Reiter [116] performed a validation analysis of wind around a single building, around a group of buildings and a dense urban environment, to select the right turbulence model and other parameters. The results of the validation test were compared to a wind tunnel test published by Wiren [118]. The structure is $12 \mathrm{~m}$ high and $18 \mathrm{~m}$ wide. The configuration of the system is shown in Figure 9.7; where $\mathrm{L}=80 \mathrm{~m}$ wide. Important factors that affect the simulation are:

- The blocking ratio should not exceed 3\% (the ratio between the vertical surfaces of the building exposed to the wind and the surface from the air inlet surface).

- The minimum length of the simulation field is $5^{*} \min (\mathrm{L}, 2 \mathrm{H})$ upstream of the building and $8^{*} \min (\mathrm{L}, 2 \mathrm{H})$ downstream. (L is length of building and $\mathrm{H}$ is height).

- When wind speed is low, thermal effects can influence the air movements

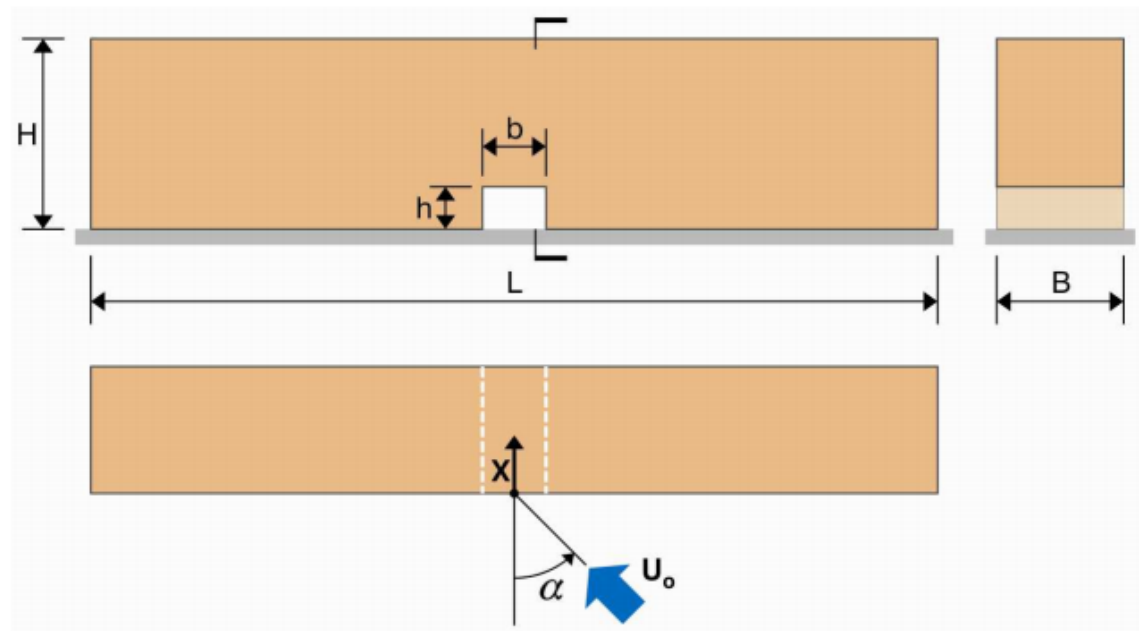

Figure 9.7: Configuration of the simulation for flow past a single building [116]

The wind profile that was used in the CFD model is equal to the one of the wind tunnel test, and had a profile of $U=10(z / 2)^{0.125}$; where $\mathrm{U}$ is the wind speed, $\mathrm{z}$ is the height at which the wind speed is calculate, and the turbulence intensity is $14 \%$. One of the simplest descriptors of atmospheric turbulence is the turbulence intensity [113]. It is the ratio of the standard deviation of a wind speed $\sigma$ component to the mean wind speed $U ; \sigma / U$. 
For the simulation, 1,384,836 cells were used for the mesh. Figure 9.8 shows the results from the CFD models in comparison to Wiren wind tunnel test results. The CFD models that were considered are based on the realizable 2nd order, $k-\epsilon$ 2nd order and the RSM 2nd order models. The findings are as follows.

The standard $k-\epsilon$ shows that the maximum discomfort intensity is the the first third of the passage; which is similar to the wind tunnel test results, however, the position of maximum discomfort is not simulated correctly. The realizable $k-\epsilon$ model improves the estimation of the location of the discomfort estimation; however still underestimates the location of the maximum discomfort. The Reynolds stress model gives the most accurate results, since it identifies the critical area correctly and the value of the maximum wind discomfort [116].

Therefore, the Reynolds stress model will be used for analysis in the scope of this thesis, since it is the most accurate for a building type configuration.
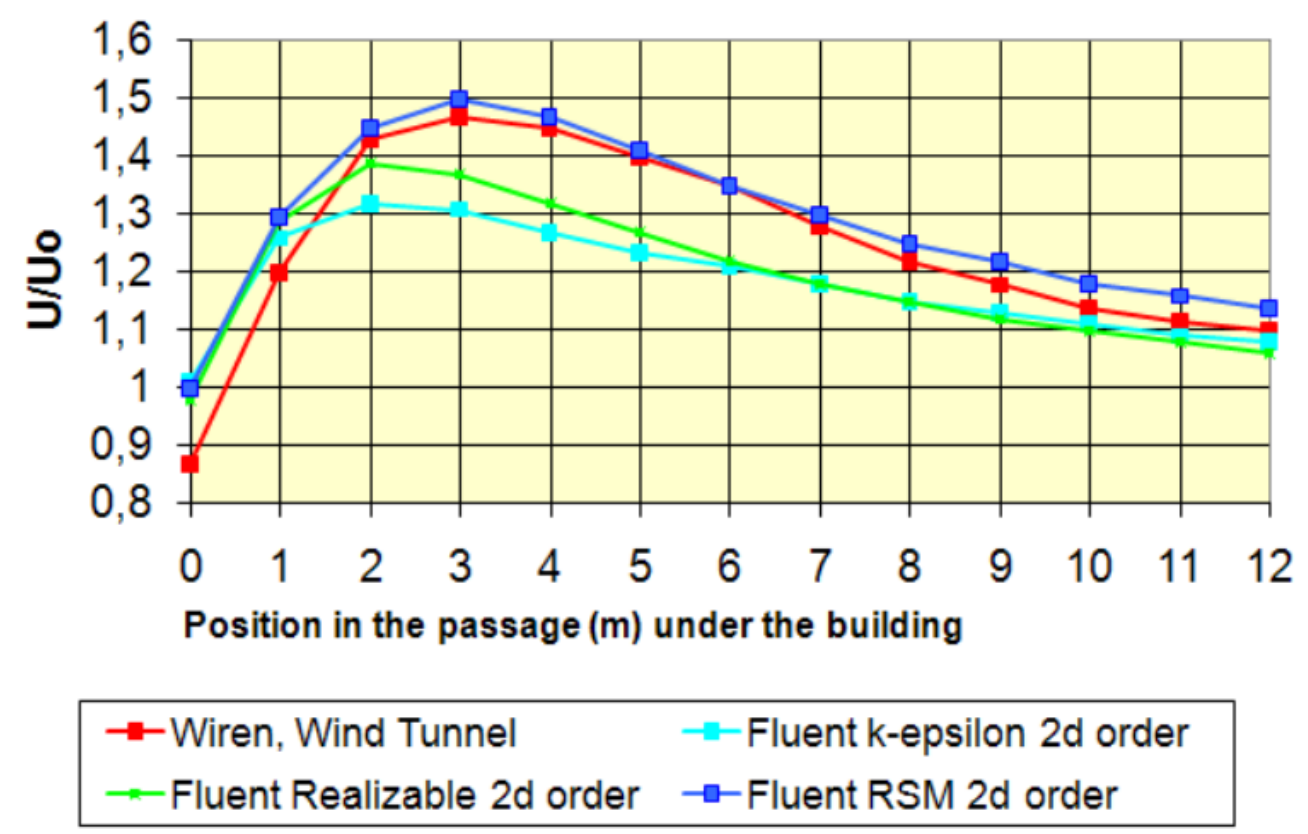

Figure 9.8: Comparison of results from FLUENT and from the wind tunnel test [116]

\subsubsection{CFD Model}

\section{Problem Definition}

Considering a frame of 10 metres height and 4 metres width, and a wind speed of $40 \mathrm{~m} / \mathrm{s}$ running for 3 seconds. The frame is shown in Figure 9.9a, along with 
the external surface boundary. The surfaces are labelled to show the inlet, outlet and the walls. The frame walls are highlighted in Figure 9.9b. The wind is applied from the inlet with the required velocity. The structure of the frame is the empty space in the figure, where the walls have the material properties of steel or concrete, as desired. The no-slip conditions are applied on the frame wall. Since turbulence is expected, the unsteady state flow is selected for the analysis.

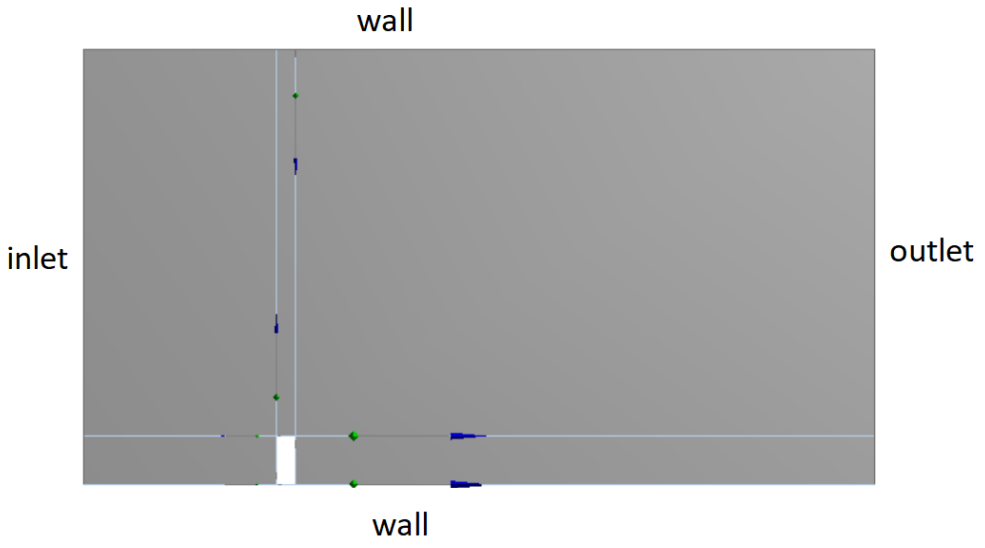

(a) Geometry of frame in Boundary

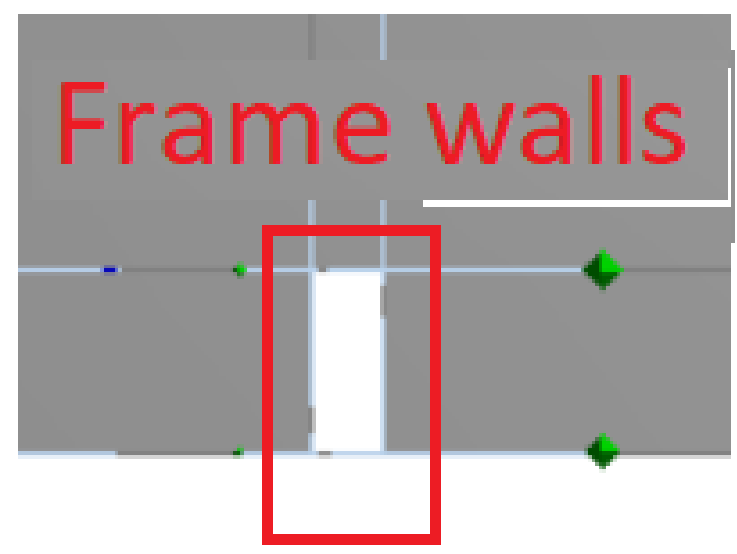

(b) Denotation of Frame walls in CFD Model

Figure 9.9: Boundary conditions

\section{Mesh}

The mesh refinement is very important to produce good quality precision [116]. It is recommended that the mesh is refined at the pedestrian level and locations where strong wind gradients are expected. The mesh for the surface is generated by using equally sized quadratic elements since the flow is aligned with the 
geometry, and the geometry is not complex. The mesh is displayed in Figure 9.10 .

The mesh can be better generated for a more efficient mesh. For instance, there could be finer mesh refinement around the structural body and more dense closer to the boundary walls and the outlet. However, a face mesh with equally sized elements was used for simplicity and demonstration purposes. The mesh is showed to have good results for capturing the air flow around the structure and is therefore selected for all models in this chapter.

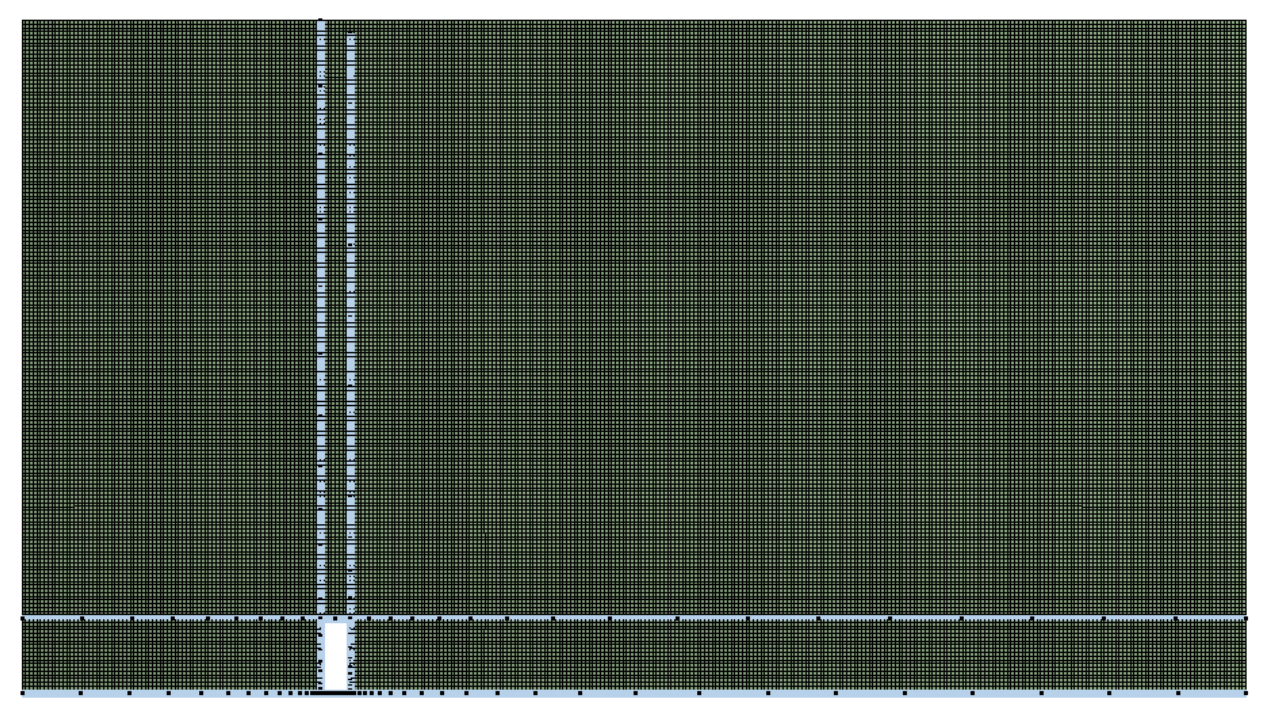

Figure 9.10: Mesh for flow around structure

\section{Time step size}

To run for three seconds, the time step size is 0.01 seconds, with a total number of 300 time steps. From the CFD model the output required is the force applied on each wall, in the $\mathrm{x}$ and $\mathrm{y}$ direction. The output will result in an average force along each wall for every iteration. So there will not be a different value for the force at each node along a wall.

Also, it is important to mention that the walls of the building are rigid and will not deflect due to the applied wind load. Therefore, when the forces are being applied in the AEM code, they are the forces that were applied to the undeformed shape. In other words, if a node "a" was to move due to the wind load, the load applied in later iterations is the load applied at that specific time step with the 
original configuration of the node.

The solution model used for modelling the 3 second gust is the 'Reynolds stress model', with a coupled pressure-velocity scheme and Second Order Upwind for the turbulent kinetic energy and the turbulent dissipation rate.

\subsection{3 $40 \mathrm{~m} / \mathrm{s} 3$ second gust wind}

Applying a wind speed of $40 \mathrm{~m} / \mathrm{s}$ in the x-direction from the inlet wall, the results are displayed as follows.

\section{CFD Results}

Figure 9.11 shows the contours of velocity, vorticity, dynamic pressure and turbulence kinetic energy for the 10x4 m frame. The residuals are shown in Figure 9.12. The residuals show that the flow is unsteady, and resemblant to turbulent flow. The force on each wall is shown in Figure 9.13. The forces are an average value at each iteration on each wall. These values are input into the AEM Gaussian code where the frame will be analysed due to the forces.

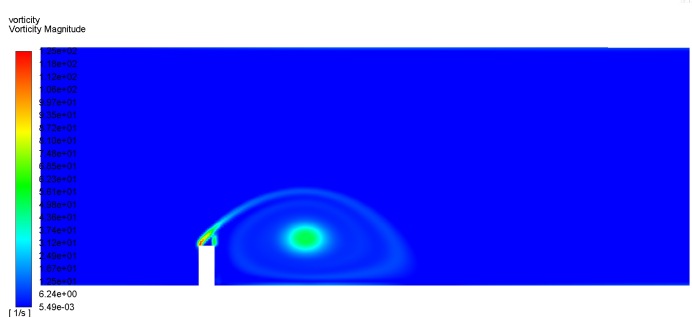

(a) Vorticity

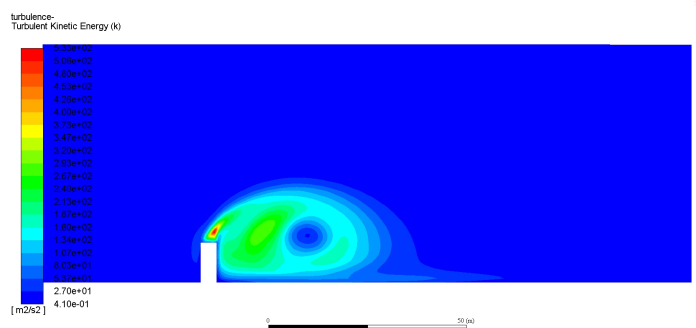

(c) Turbulence

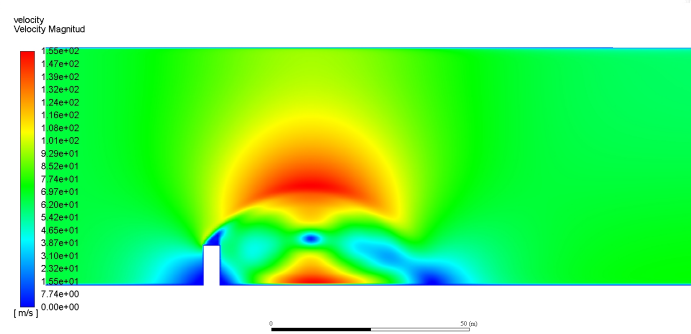

(b) Velocity

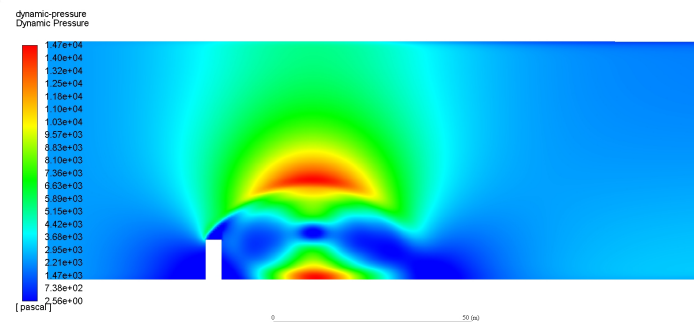

(d) Dynamic Pressure

Figure 9.11: Vorticity, velocity, dynamic pressure and turbulence kinetic energy for flow past building with $40 \mathrm{~m} / \mathrm{s}$ wind speed 

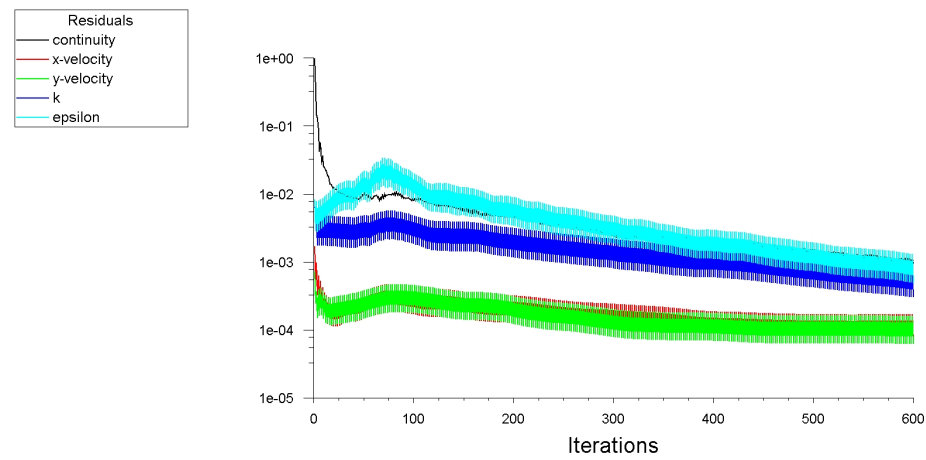

Figure 9.12: Residuals for turbulent flow

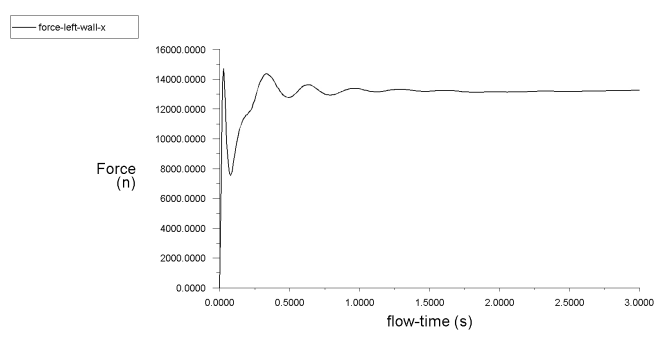

(a) Left wall - $\mathrm{x}$

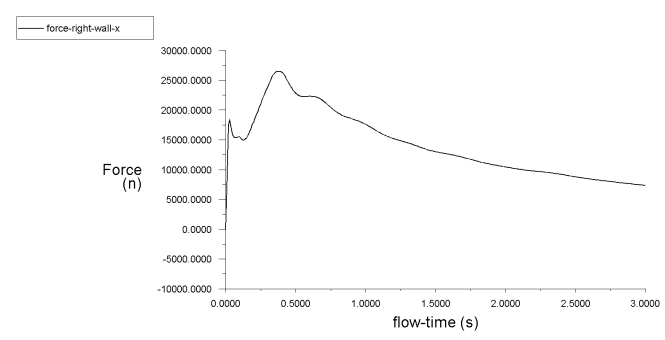

(c) Right wall - x

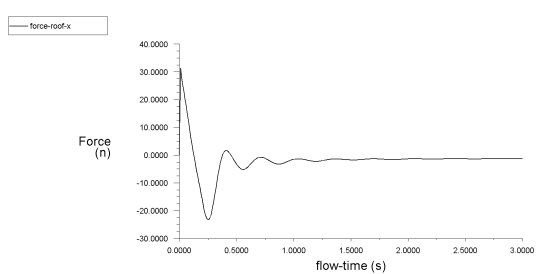

(e) Roof - $\mathrm{x}$

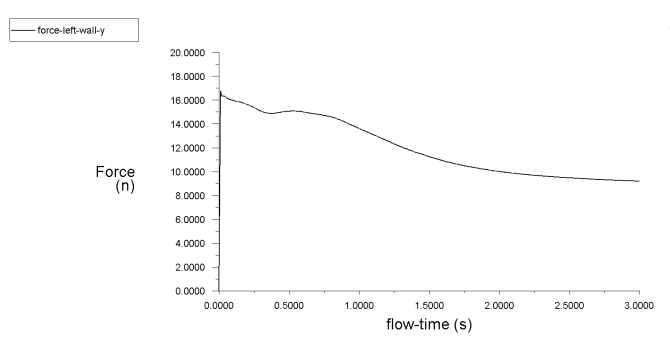

(b) Left wall - y

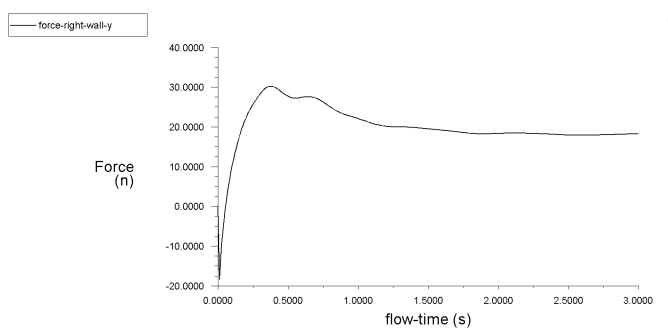

(d) Right wall - y

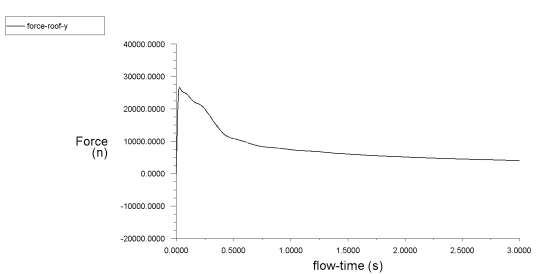

(f) Roof - y

Figure 9.13: Forces on each wall in $\mathrm{x}$ and y directions due to $40 \mathrm{~m} / \mathrm{s}$ wind speed

\section{Analysis of structure in AEM}

The forces in the $\mathrm{x}$ and $\mathrm{y}$ directions on each wall is applied to the frame in AEM. Figure 9.14 displays the displacement velocity and acceleration for a point on the left wall for the duration of the three seconds gust. The frame deflection is shown in Figure 9.15. The deflection is multiplied by $10^{3}$ so the deflection may 
be seen. There was no collapse in the structure reported. The analysis therefore shows that a frame of dimensions $10 m \times 4 m$ can with steel material properties can withstand a 3 second gust of $40 \mathrm{~m} / \mathrm{s}$. The most significant forces on the structure are the forces on the left wall in the x-direction, and the roof in the $\mathrm{y}$-direction. Table 9.2 is the maximum average force applied on each wall.
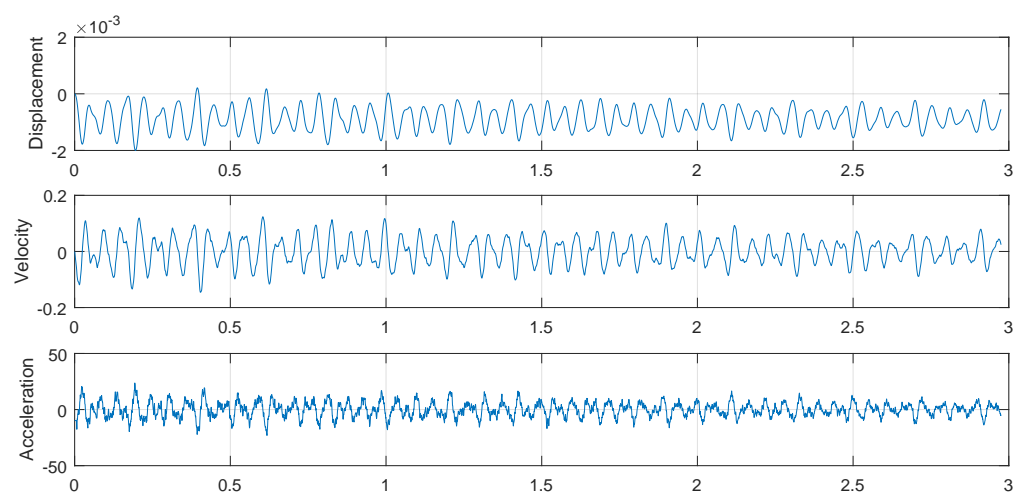

Figure 9.14: Displacement, velocity and acceleration of a single point of the frame due to the wind load of $40 \mathrm{~m} / \mathrm{s}$

Table 9.2: Maximum Average force applied on each wall of the frame

\begin{tabular}{l|r}
\multicolumn{2}{c}{ Wind speed $40 \mathrm{~m} / \mathrm{s}$} \\
\hline Location & Maximum force $[\mathrm{N}]$ \\
\hline Left wall - $\mathrm{x}$ & $14,700.0$ \\
Left wall - y & 16.8 \\
Right wall - $\mathrm{x}$ & 30.2 \\
Roof - $\mathrm{x}$ & 31.5 \\
Roof - y & $26,400.0$
\end{tabular}

\subsection{4 $70 \mathrm{~m} / \mathrm{s} 3$ second gust wind}

An extreme wind load of $70 \mathrm{~m} / \mathrm{s}$ for the three second gust is applied on the same frame structure. Figure 9.16 depicts the forces on the left and right wall in the $\mathrm{x}$ and $\mathrm{y}$ direction. It can be seen that the results lead to an eventual steady state solution where the forces remain constant. In Figure 9.17 the contours of velocity, turbulence and vorticity are presented. The deflection of the frame at several seconds are shown in Figure 9.18. From the deflection it can be seen that the structure starts to collapse at 0.35 seconds near the right boundary nearest to the ground. Eventually there is a total collapse of the frame. 


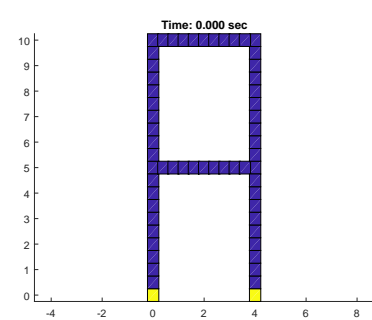

(a) $0 \mathrm{sec}$

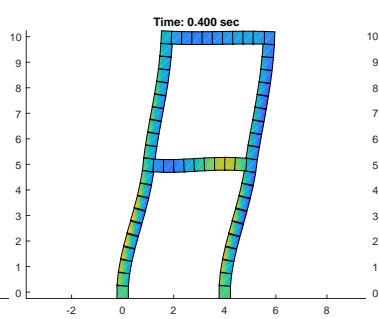

(b) $0.4 \mathrm{sec}$

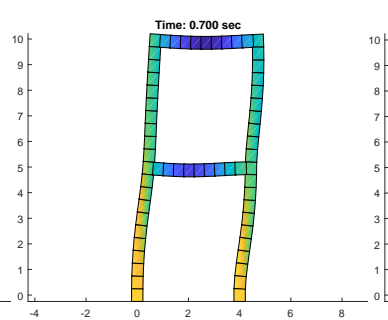

(c) $0.7 \mathrm{sec}$

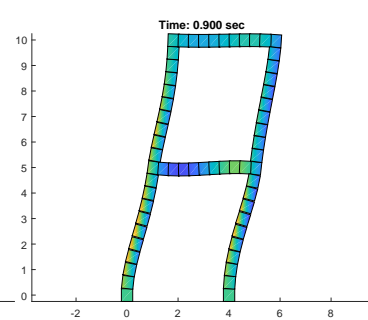

(d) $0.9 \mathrm{sec}$

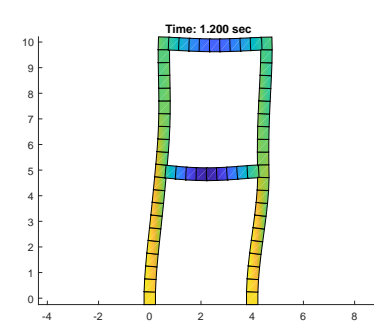

(e) $1.2 \mathrm{sec}$

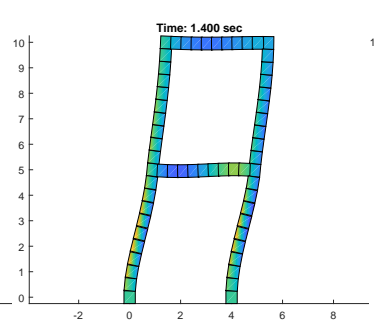

(f) $1.4 \mathrm{sec}$

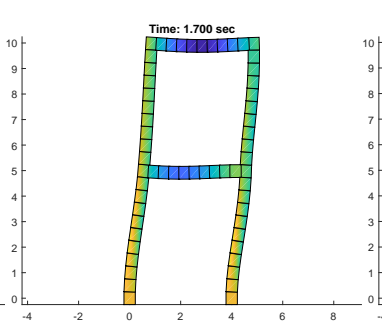

(g) $1.7 \mathrm{sec}$

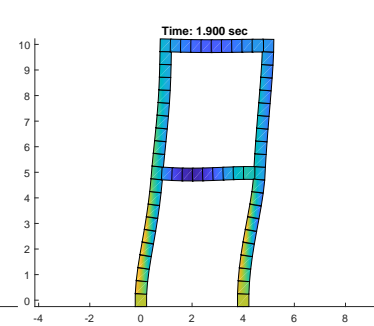

(h) $1.9 \mathrm{sec}$

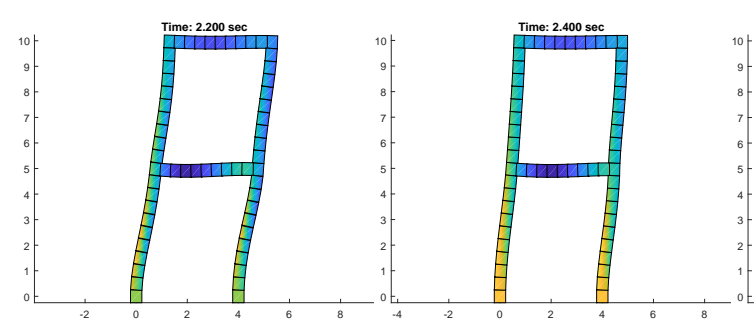

(i) $2.2 \mathrm{sec}$

(j) $2.4 \mathrm{sec}$

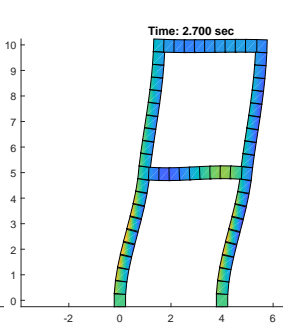

(k) $2.7 \mathrm{sec}$

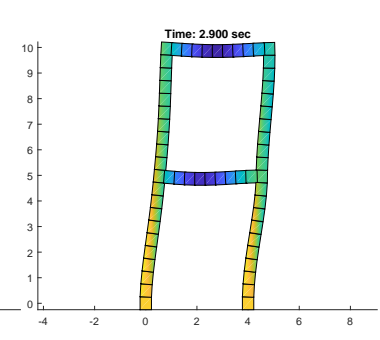

(l) $2.9 \mathrm{sec}$

Figure 9.15: Frame displacement factored by $10^{3}$ for $3 \mathrm{sec}$ gust $40 \mathrm{~m} / \mathrm{s}$ wind speed

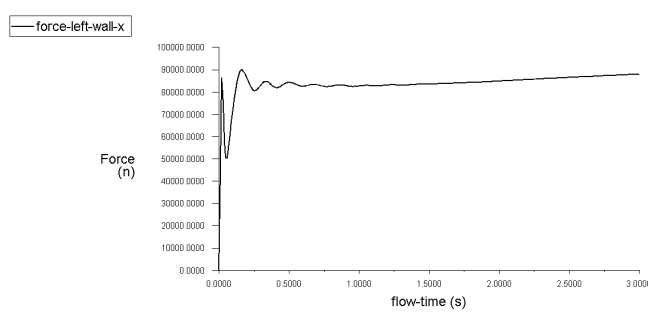

(a) Left wall $\mathrm{x}$

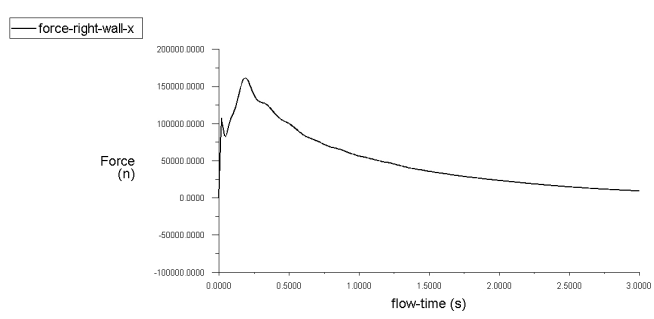

(c) Right wall $\mathrm{x}$

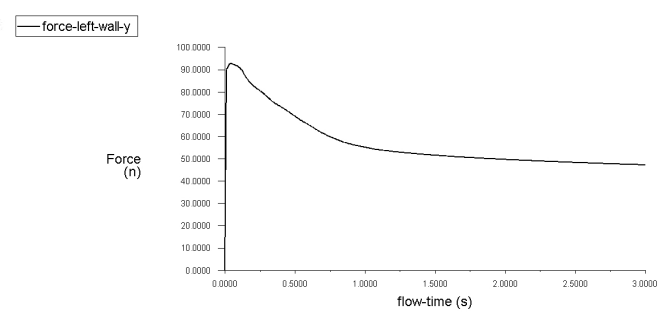

(b) Left wall y

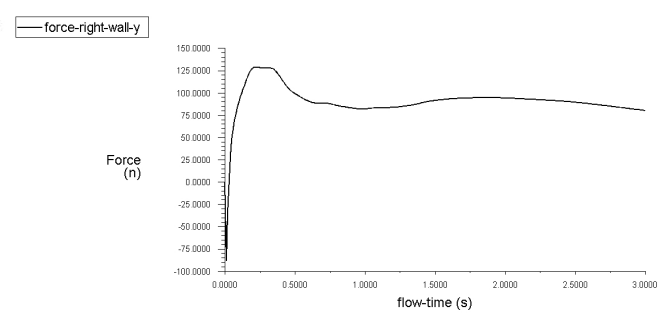

(d) Right wall y

Figure 9.16: Forces on left and right wall due to $70 \mathrm{~m} / \mathrm{s}$ wind speed 


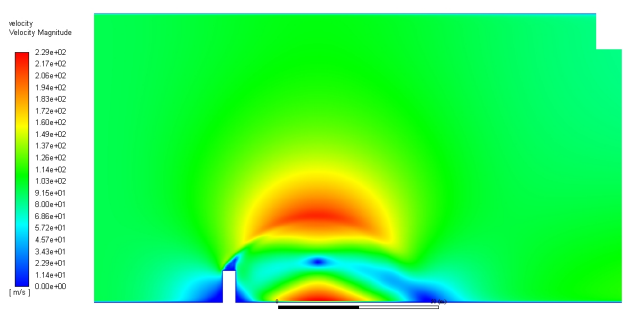

(a) Velocity contours

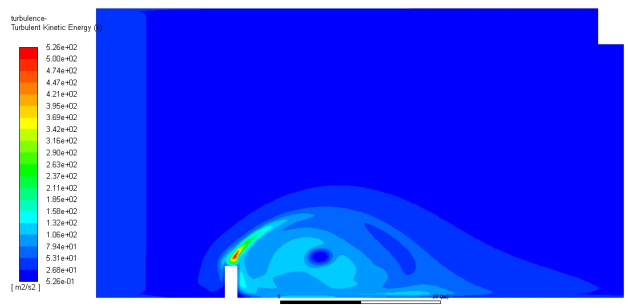

(c) Turbulence contours

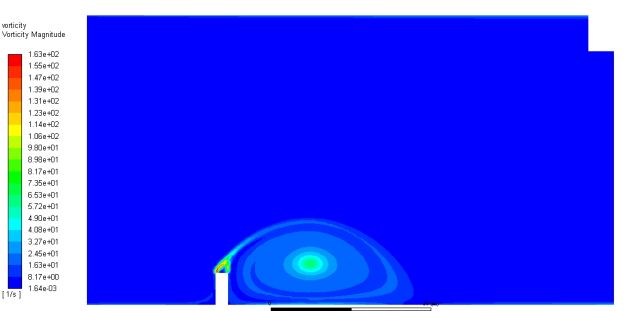

(b) Vorticity contours

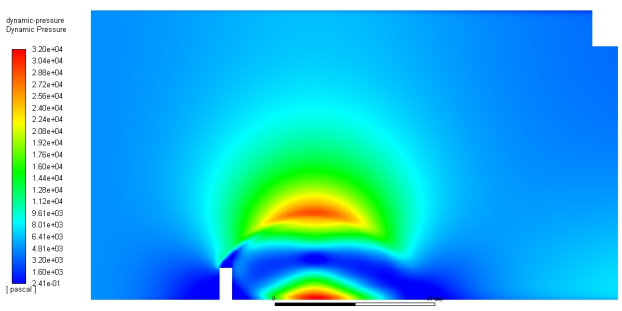

(d) Dynamic Pressure contours

Figure 9.17: Contours due to the $70 \mathrm{~m} / \mathrm{s}$ wind speed, 3 second gust

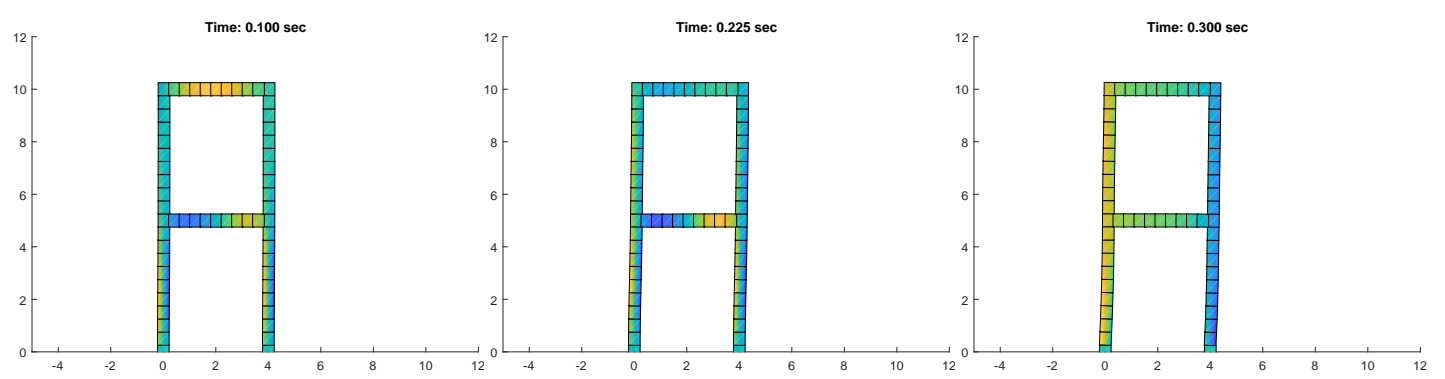
(a) $0.1 \mathrm{sec}$
(b) $0.225 \mathrm{sec}$
(c) $0.3 \mathrm{sec}$

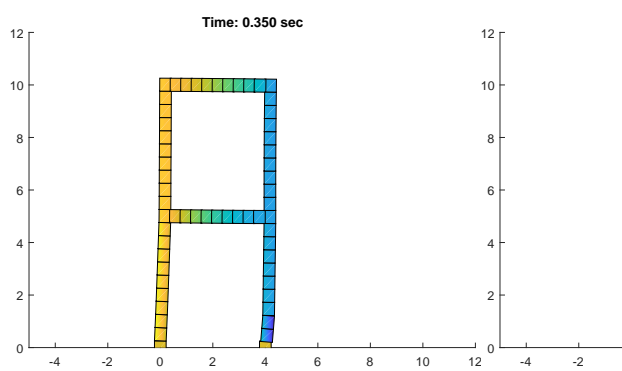

(d) $0.35 \mathrm{sec}$

Time: 0.400 sec

Time: 0.500 sec

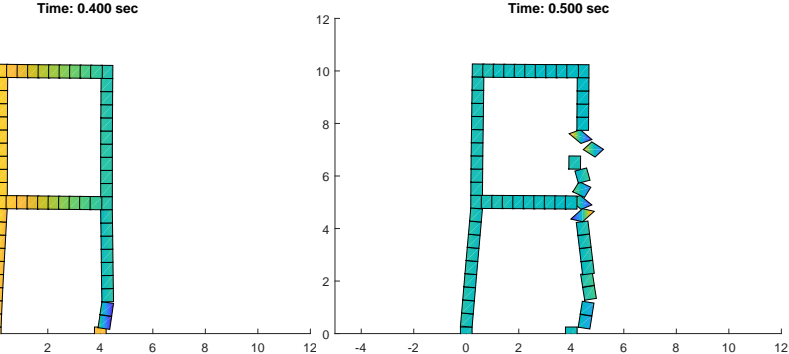

(e) $0.4 \mathrm{sec}$

(f) $0.5 \mathrm{sec}$

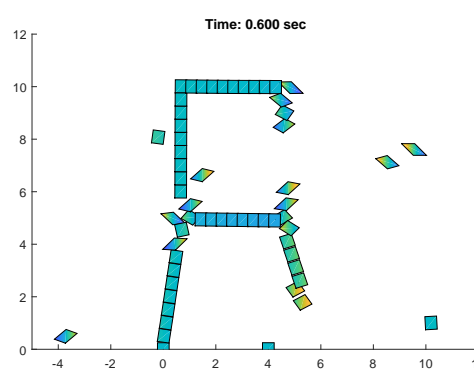

(g) $0.6 \mathrm{sec}$

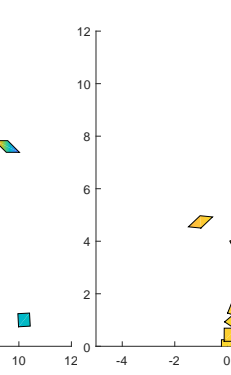

Time: 0.700 sec

Time: 0.800 sec

Figure 9.18: Collapse of frame subject to $70 \mathrm{~m} / \mathrm{s}$ wind speed 


\subsection{Loads from surrounding structures}

It is also important to consider the change in the wind effect due to surrounding structures. An analysis done by Aly showed that the existence of short buildings in the upstream wind increases the turbulence in the region [119]. Since the scope of this thesis only covers 2D analysis, only 2D CFD models are examined.

Analysing the structure in the middle after a $40 \mathrm{~m} / \mathrm{s} 3$ second gust. The geometry model is shown in Figure 9.19 for three adjacent buildings. Figure 9.20 represents the surface mesh around the buildings. A standard mesh with equal sized elements was used, for simplicity. Figure 9.21 shows the forces on all the

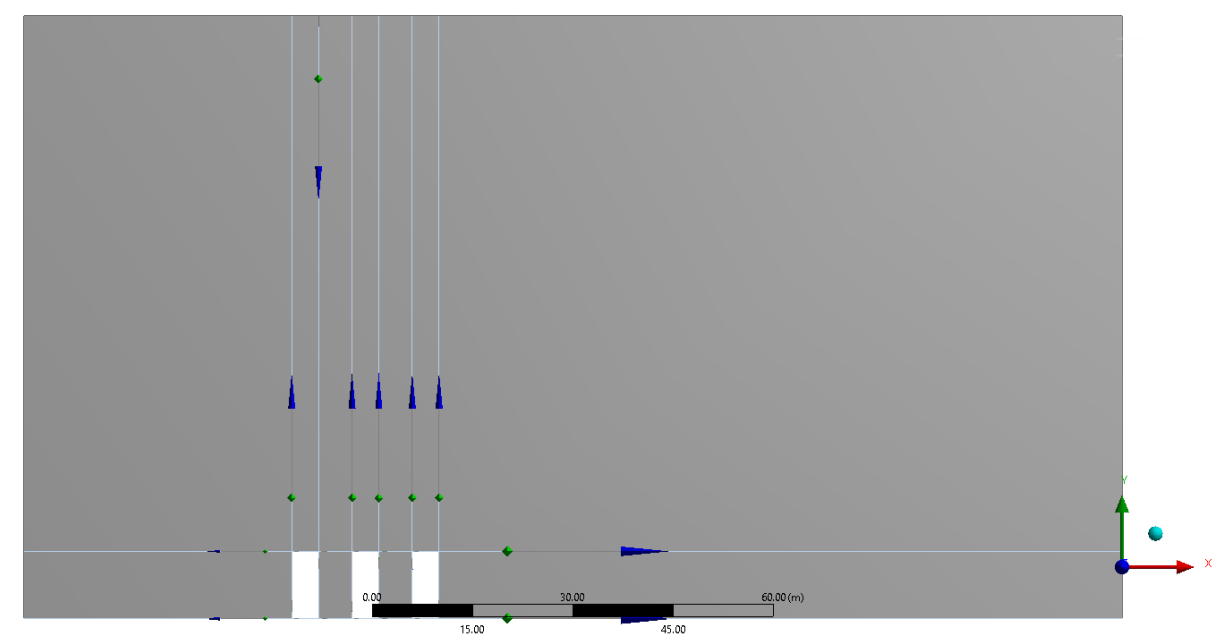

Figure 9.19: Geometry model for three adjacent buildings

building walls for the third structure.

Figure 9.22 shows the vorticity, velocity, turbulence kinetic energy and the dynamic pressure contours.

Figure 9.24 shows the deflection of the frame subject to the 3 second gust wind speed of $40 \mathrm{~m} / \mathrm{s}$ while being affected by neighbouring structures. 


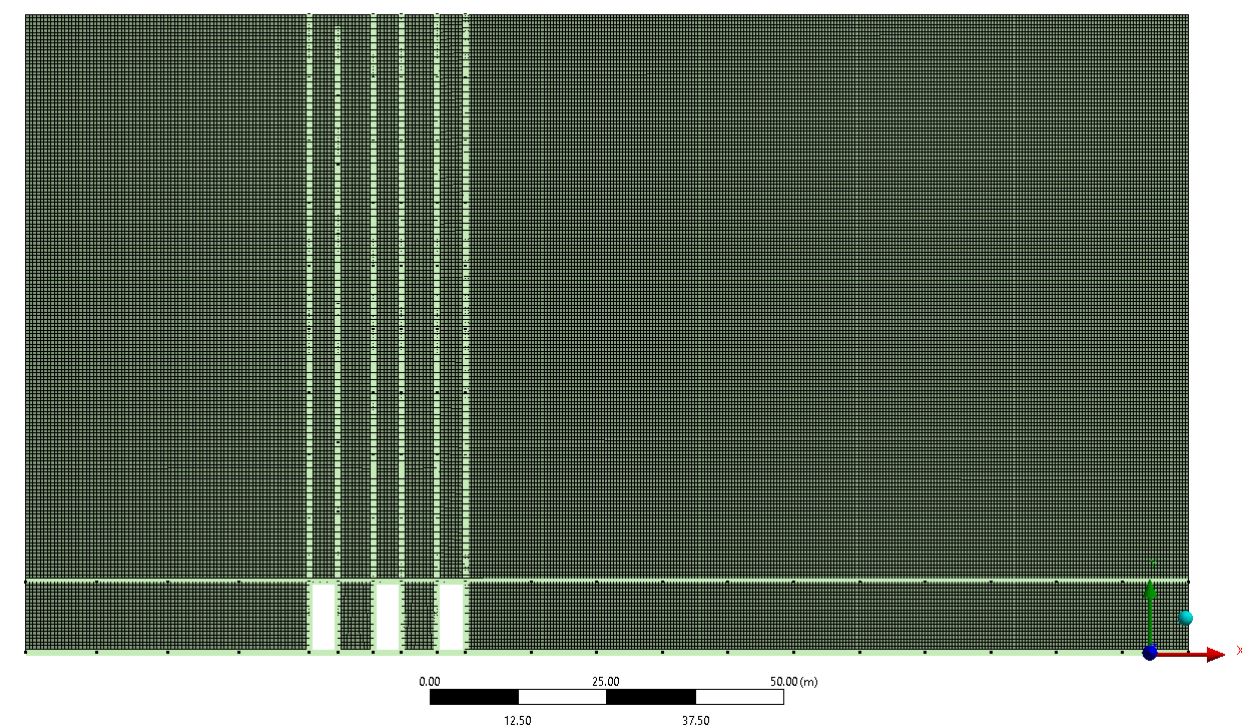

Figure 9.20: Mesh for three adjacent buildings

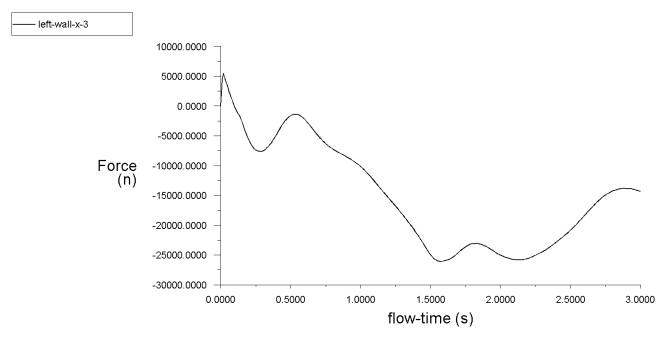

(a)

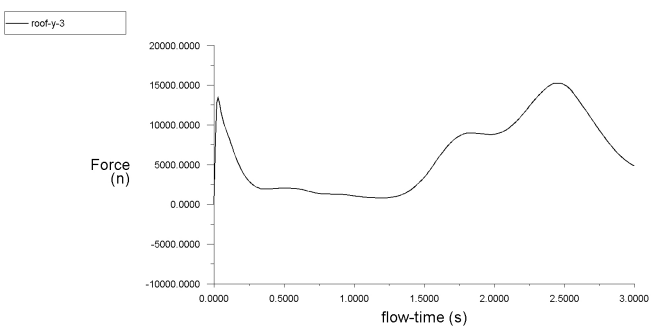

(c)

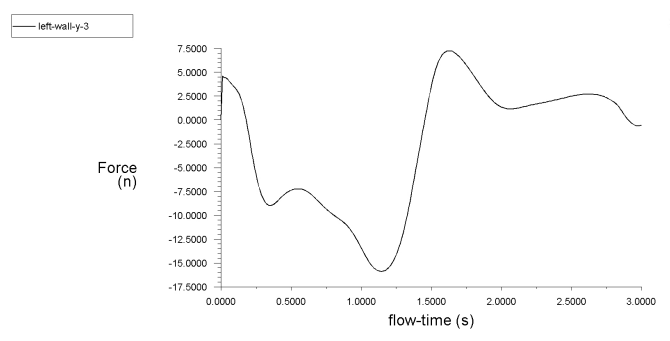

(b)

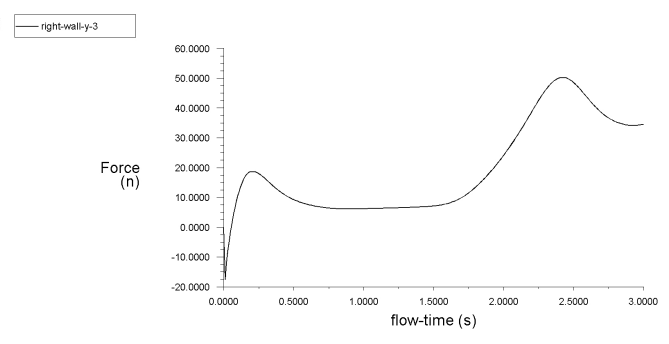

(d)

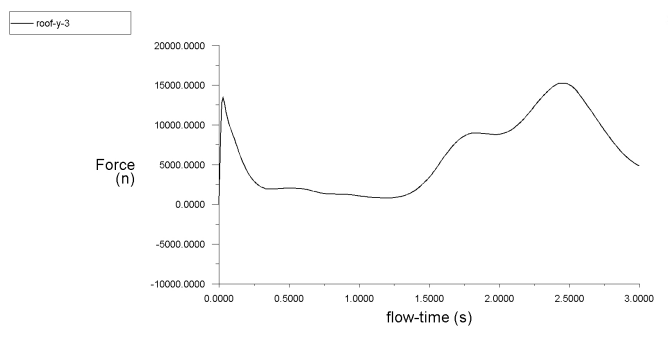

(e)

Figure 9.21: Forces on all the walls for the third building with 3 second gust wind $40 \mathrm{~m} / \mathrm{s}$ 


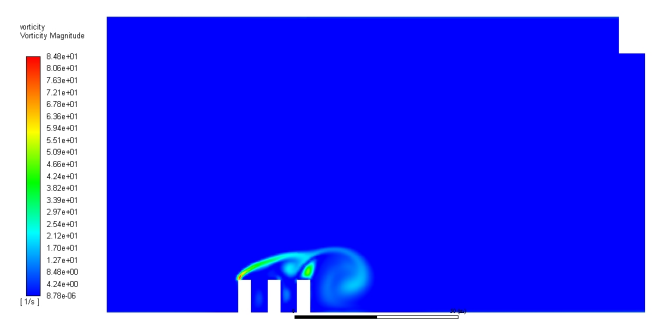

(a) Vorticity

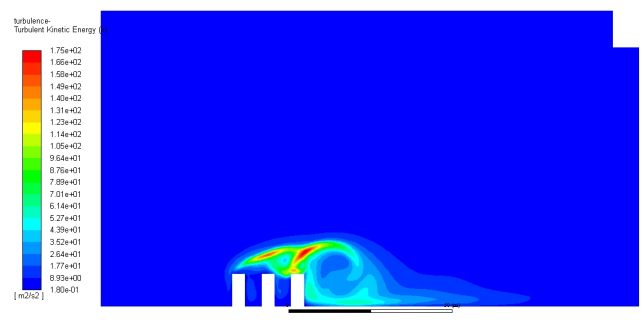

(c) Turbulence

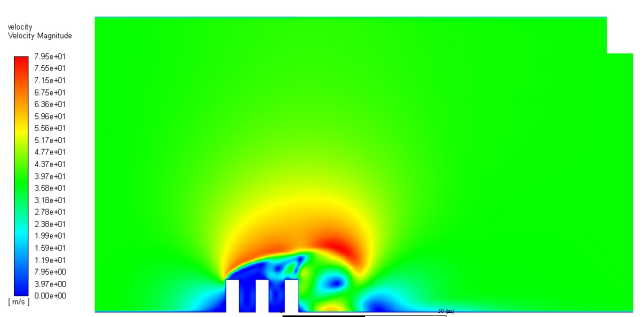

(b) Velocity

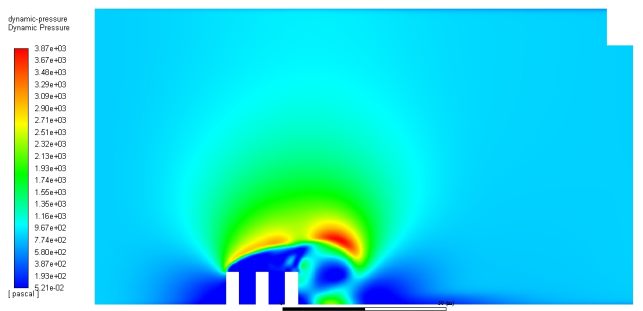

(d) Dynamic Pressure

Figure 9.22: Vorticity, velocity, turbulence and dynamic pressure contours of three adjacent buildings with $40 \mathrm{~m} / \mathrm{s}$ wind speed
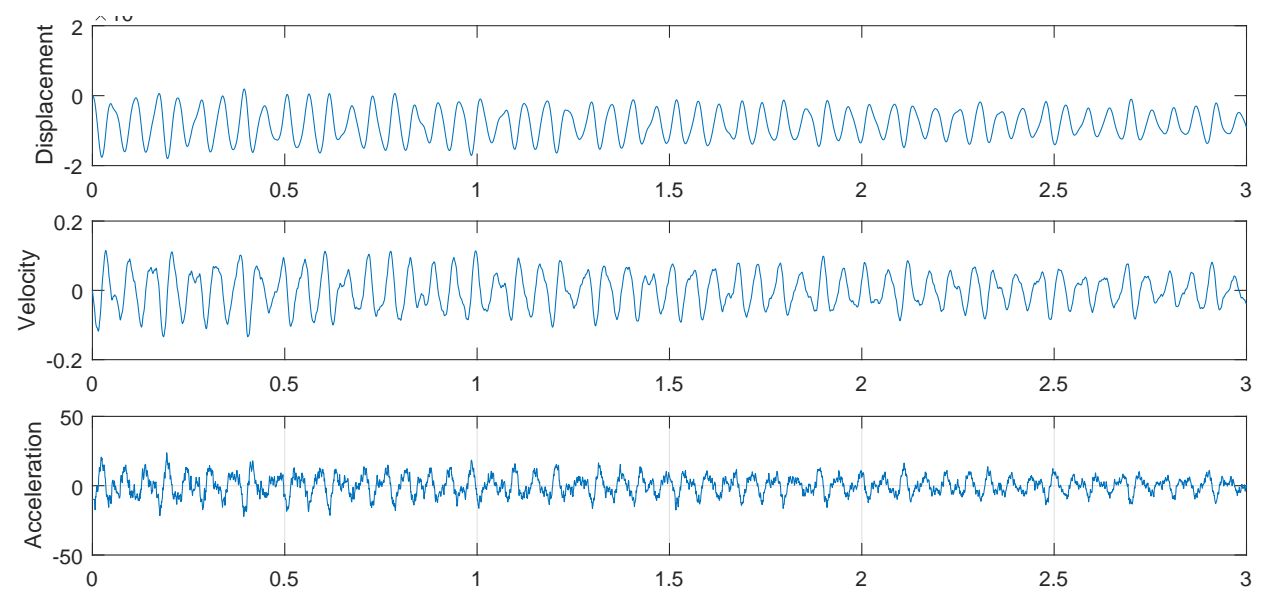

Figure 9.23: Displacement velocity and acceleration for the third building at 40 $\mathrm{m} / \mathrm{s} 3$ second gust 


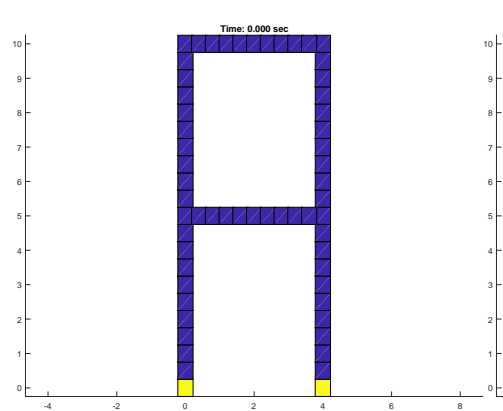

(a)

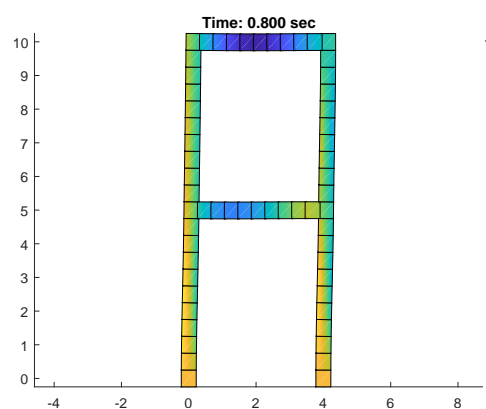

(d)

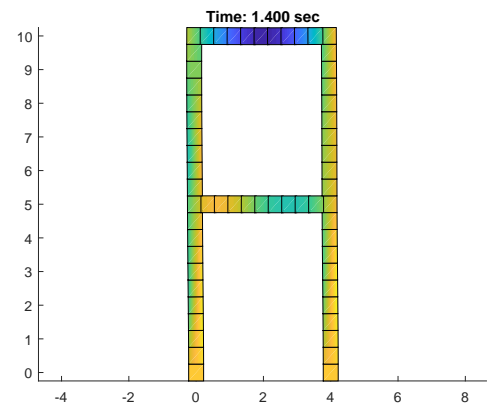

(g)

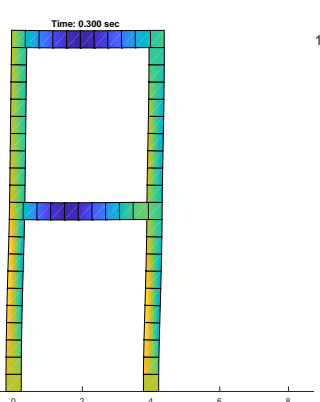

(b)

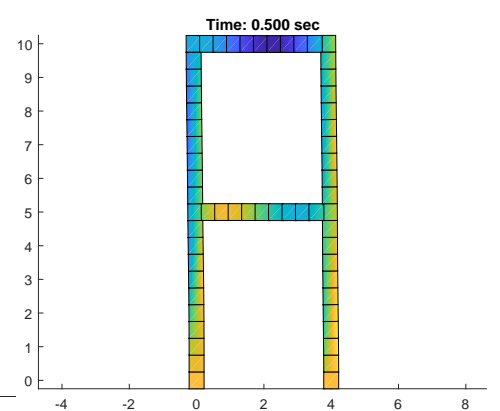

(c)

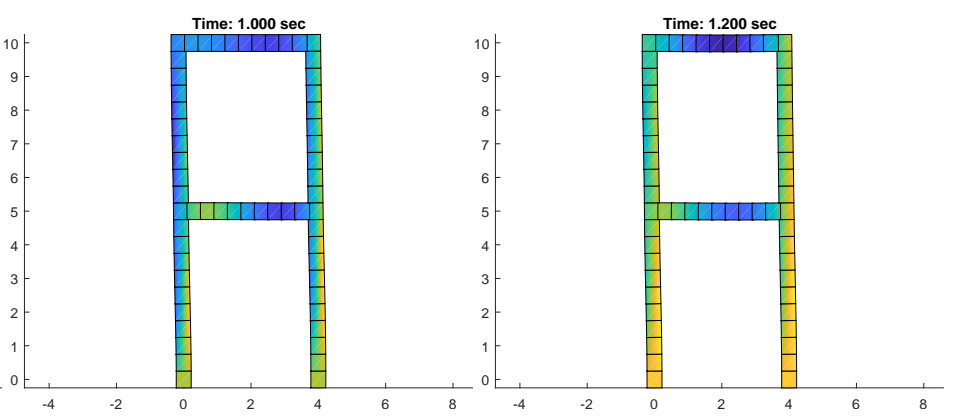

(e)

(f)

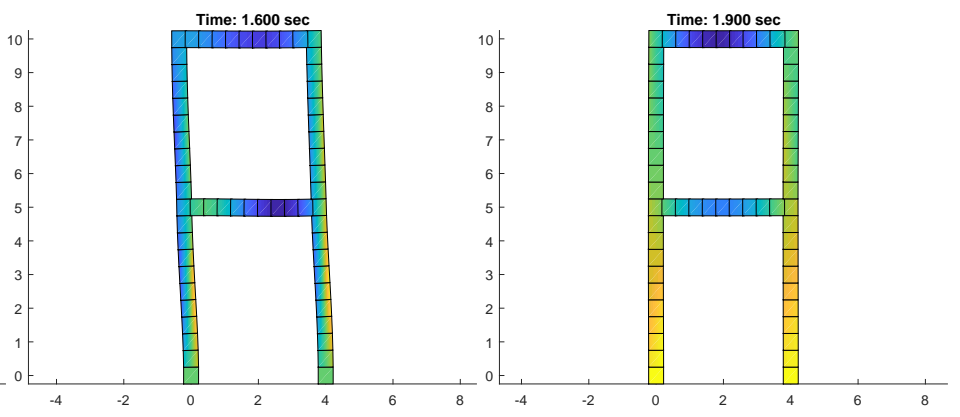

(h)

(i)

Figure 9.24: Frame deflection subject to $40 \mathrm{~m} / \mathrm{s}$ wind; effect of neighbouring buildings 


\subsection{Conclusion}

The analysis of structures subject to wind loading conditions was performed in this chapter. The wind analysis is performed in FLUENT, where there is a velocity inlet that presents the desired wind speed for analysis. The structure undergoes the wind analysis for 3 seconds to replicate the 3 second gust. At each time step an average force from each wall is recorded. The forces are then applied to the frame in the MATLAB code, to perform the deflection analysis of the structure using the Gaussian springs based AEM.

The purpose of using FLUENT to emulate the wind behaviour is to obtain relatively realistic wind loads from 3 second gust behaviours, and not to analyse how the wind should behave. Also it is simply used to demonstrate different applications of loads to be included in the AEM analysis.

Although only 3 second gusts were used to analyse the structures, longer periods of time can easily be modelled. The same models will be used, except they will be run for longer. It was simply for demonstration purposes and the scope of this thesis that 3 second wind gusts are chosen for analysing structures under extreme weather conditions.

It is also important to mention that the FLUENT and Matlab code are not directly coupled, because during the Ansys analysis, it is expected that the structure should deform after being subject to the extreme wind. However, this is not the case. In Ansys the walls are completely rigid, and the forces obtained are based on the original configuration of the frame, not its deformed shape.

The results from this chapter showed successful modelling of the collapse of structures subject to extreme wind loading. 


\section{Part IV}

\section{Conclusions}




\section{Chapter 10}

\section{Concluding Remarks}

\subsection{Conclusions}

Based on a comprehensive review of computational methods used for modelling the progressive collapse of structures, the AEM is deemed to be one of the simplest schemes with acceptable accuracy.

The AEM is implemented as a discretisation of structural elements into rectangular rigid bodies that are connected along the edges of the elements with pairs of shear and normal springs. The material behaviour of the structural elements are modelled through the springs stiffness. Various advantages arise from the use of springs between elements. Firstly, when a spring exceeds a specific yield strain and is considered to have failed then the spring can be easily removed from the system to represent the discontinuity between elements. Another advantage is the capability of defining unique material properties to specific springs at specific locations. This grants a straightforward implementation of multiple materials within the same element, such as modelling reinforced concrete. The AEM also exhibits a good advantage in adequately modelling both the continuum and discrete elements behaviour. Before any element separation occurs the structural system behaves as a continuum, while as springs start to fail and elements detach, discrete element behaviour is considered.

The deflection and internal stresses of several structural beams are assessed using the conventional AEM and it is evident that the computational efficiency of the method is inadequate since a sizable amount of elements and springs per 
element is required to achieve a specific level of accuracy.

In this thesis, a modification to the AEM has been presented, where the number of springs between elements is optimised using the Gaussian quadrature to locate the optimal location of springs. The findings of the application of the Gaussian AEM to simple structures for linear and nonlinear material behaviour are presented in the following section.

\subsubsection{Linear and Nonlinear Gaussian AEM}

As mentioned earlier, the Gaussian AEM utilises Gaussian quadrature weights and locations for defining spring locations across a pair of rigid elements.

\section{Linear Gaussian AEM}

In the linear elastic case structural beams were subject to a point load. The expected normal stress along a rectangular beam cross-section is a linear line with a value of zero at the neutral axis. Using the Gaussian springs it was found that exactly 2 gaussian springs were needed to significantly improve the analysis and reduce the computational cost. This simple change showed that using the Gaussian AEM significantly less springs were required for attaining an acceptable level of accuracy.

\section{Nonlinear Gaussian AEM}

Nonlinear material behaviour was then implemented in the analysis. The material nonlinearity was represented by a 1D Hardening plasticity algorithm. Based on the yield criteria springs were classified as elastic or elasto-plastic and the corresponding spring stiffness were calculated. A cantilever beam was analysed using the Gaussian AEM and the FEM solution from ANSYS, and the results showed good accuracy.

In the case of a rectangular cross-section undergoing elasto-plasticity, the stress along the cross-section is expected to experience plasticity at the top and bottom fibres of the cross-section and elasticity along the middle of the crosssection. Hence, the stress along the cross-section is split into three parts (plasticelastic-plastic). 10 Gaussian springs were used to appropriately capture the tran- 
sition between the plastic and elastic regions along the rectangular cross-section. The Newton Raphson iteration scheme was implemented in the Gaussian AEM with rate dependent Hardening plasticity. Remarkably a quadratic convergence in the analysis was observed.

\section{Adaptive Gaussian AEM}

Since each part of the elasto-plastic material behaviour can be represented linearly, 2 Gaussian springs are enough to explicitly represent each region. The exact number of springs needed to represent the stresses along a cross-section is known, an adaptive technique was formulated to exploit this, where only 6 springs were required for the nOnlinear analysis. The adaptive scheme is as follows. At each load increment, the first NR iteration has an initial number of 10 Gaussian springs. After the first iteration the strain and stress of each spring is computed from the deflection obtained. Based on the yield criteria, springs are classified as elastic or elasto-plastic. If all the springs between a pair of elements were elastic, then the number of springs were changed from the initial 10 springs to 2 Gaussian springs. However, if the springs were found to be elasto-plastic, then the number of springs between an element was changed to 6 . The exact location at which the stress changes from elasto-plastic to linear is calculated by obtaining the intersection of the two lines. Those intersection points were referred to as transition points. For the remaining NR iterations, the new springs configuration is used. At the next load increment the initial number of springs is returned to 10 and the process is repeated again. Using the transition springs and the adaptive technique significantly reduced the computational cost since the number of springs in the whole system was reduced at each time step. Since the linear and nonlinear material behaviour was successfully modelled using the Gaussian AEM and the springs distribution was optimised, modelling the material damage was then considered.

The novel use of the Gaussian springs shows a phenomenal revelation since the size of the model significantly decreases, and therefore optimising the number of springs required per element. Only 2 Gaussian springs are required for linear elements, and 6 Gaussian springs for nonlinear elements. 


\subsubsection{Modelling Damage Fragmentation and Progressive Collapse in AEM}

In this thesis, damage was considered as a type of softening material behaviour. A return mapping algorithm for the softening material behaviour was developed to predict and correct the stress based on a strain increment. First, the strain softening algorithm was verified to show that the return mapping behaviour works correctly then it was implemented to the Gaussian AEM code.

Since the material model was produced, fracture was then considered. If a spring exceeds its failed limit, then the spring stiffness is set to zero so as to "remove" the failed spring from the system. The problem is that having failed springs in the system results in having zeros in the global stiffness matrix. This resulted in no convergence in the NR due to the singularity of the stiffness matrix. Also, the eigen values for the system are negative after the failed point is surpassed. A solution to these problems is using a dynamic model for analysis.

Different time-stepping methods were compared for finding the most appropriate method for use in modelling the damage and progressive collapse of structures. The Newmark-Beta method, the linear acceleration method and the Central difference method were compared. The CDM was chosen as the most appropriate method for this scope since it is explicit, and no iterative procedure is required because all the constitutive variables are available from computations at previous time steps. The drawback is the requirement of a small increment of time step. However, the damping matrix and mass matrix were diagonal, and the stiffness matrix did not need to be inverted at every time step since there was no NR iteration required, so ultimately even though the number of total time steps is larger, the overall computational cost is still considered smaller.

Since the softening model can represent both linear and nonlinear material properties, then implementing the softening model to the CDM allowed to produce a code with both linear and nonlinear material behaviour. A modification to the algorithm was required however, since the dynamic model did not require the calculation of the stiffness matrix in every iteration, rather only the internal forces were needed. Secondly, since the CDM was used no NR iteration scheme 
was needed for nonlinear analysis.

The damage and fragmentation model was then used for application of frames subject to earthquake loads, and to extreme wind gusts. The progressive collapse of each of those structures due to the earthquake loads, and sometimes including external horizontal loads to simulate effect of nearby objects or impact, were analysed, and the progressive collapse of the structures were clearly represented.

Frames were then subject to 3 second wind gust loads. The 3 second wind was first simulated using CFD in ANSYS Fluent. The analysis for different wind speeds and different structures was performed. After the analysis was complete, the forces that were generated on the walls were exported to the MATLAB code, where the structure would then be analysed for the external forces. The progressive collapse of the structures was successfully shown in the cases that the wind speed was very large.

\subsection{Concluding Remarks}

Based on the findings in this thesis, the following final remarks can be made:

1. The efficiency and the accuracy of the AEM is successfully improved by using the Gaussian distribution for optimising the springs locations.

2. Only 2 linear Gaussian springs, and 6 nonlinear Gaussian springs are needed between a pair of elements.

3. An adaptive springs procedure is implemented to distribute element springs based on current state of material behaviour (elastic or elasto-plastic) for an overall reduced computational cost.

4. A softening return mapping algorithm is developed for representing damage in materials.

5. A time integrating technique is required when elements in structures exceed their failed limits and separation occurs. The element separation causes stiffness matrix singularity.

6. The progressive collapse of structures is successfully modelled using the Gaussian-AEM and softening material behaviour, with implementation to structures subject to earthquakes and extreme wind loads. 


\subsection{Recommendations for further research}

- Include contact between elements. Including the contact between elements can give more realistic results when the collapse occurs, and especially if effect of collapse of surrounding structures are considered.

- Implementation to 3D AEM. The Gaussian AEM should be expended to application of 3D elements, this can allow 3D models of frames to be analysed for collapse.

- Flow past buildings in 3D. Based on the extreme wind application chapter, it is interesting to model the effect of wind due to surrounding structures from all directions. 
Part V

\section{References}




\section{Bibliography}

[1] Natural Catastrophes - Our World in Data. Sept. 15, 2018. URL: https:// ourworldindata.org/natural-catastrophes (visited on 09/15/2018).

[2] Eisuke Ikuta et al. "Measurement of the human body damage caused by collapsed building". In: 13th World Conference on Earthquake Engineering. 2004.

[3] John L Gross and William McGuire. "Progressive collapse resistant design”. In: Journal of Structural engineering 109.1 (1983), pp. 1-15.

[4] Xinzheng Lu, Xuchuan Lin, and Lieping Ye. "Simulation of Structural Collapse with Coupled Finite Element-Discrete Element Method". In: Computational Structural Engineering. Ed. by Yong Yuan, Junzhi Cui, and Herbert A. Mang. Dordrecht: Springer Netherlands, 2009, pp. 127-135.

[5] XZ Lu et al. "Numerical simulation for the progressive collapse of concrete building due to earthquake". In: Proc. the 14th World Conference on Earthquake Engineering. October. 2008, pp. 12-17.

[6] Kimiro Meguro and Hatem Tagel-Din. "Applied Element Method for Structural Analysis". In: Doboku Gakkai Ronbunshu 2000.647 (2000), pp. 3145.

[7] Uwe Starossek. Progressive collapse of structures. Vol. 153. Thomas telford London, 2009.

[8] J Räty. "Limiting the extent of localised failure according to SFS-EN 1991-1-7”. In: Diplomityö, Aalto Yliopisto, Espoo (2010).

[9] SM Marjanishvili. "Progressive analysis procedure for progressive collapse". In: Journal of Performance of Constructed Facilities 18.2 (2004), pp. 7985 . 
[10] Edgar V Leyendecker and Bruce Ellingwood. Design methods for reducing the risk of progressive collapse in buildings. Vol. 98. US Department of Commerce, National Bureau of Standards, 1977.

[11] Kyaw Myo Lynn and Daigoro Isobe. "Structural collapse analysis of framed structures under impact loads using ASI-Gauss finite element method". In: International journal of impact engineering 34.9 (2007), pp. 1500-1516.

[12] Hajime Okamura and Kohichi Maekawa. "Nonlinear analysis and constitutive models of reinforced concrete". In: Gihodo, Tokyo 10 (1991).

[13] JJ Jiang. "Nonlinear Finite Element Analysis of RC Structure". In: Shanxi Science and Technology Press, Xian (1994).

[14] Georgios Michaloudis et al. "Modelling structural failure with finite element analysis of controlled demolition of buildings by explosives using LS-DYNA". In: High Performance Computing in Science and Engineering'09. Springer, 2010, pp. 539-551.

[15] Peter A Cundall. "A computer model for simulating progressive, large scale movement in blocky rock systems". In: Symp. ISRM, Nancy, France, Proc. Vol. 2. 1971, pp. 129-136.

[16] Nian Qi and Jihong Ye. "Nonlinear dynamic analysis of space frame structures by discrete element method". In: Applied Mechanics and Materials. Vol. 638. Trans Tech Publ. 2014, pp. 1716-1719.

[17] Enrico Masoero et al. "Progressive collapse mechanisms of brittle and ductile framed structures". In: Journal of engineering mechanics 136.8 (2010), pp. 987-995.

[18] E Masoero et al. "DEM simulations of the progressive collapse of framed structures". In: 12th International Conference on Fracture. NRCanCANMET. 2009.

[19] A Munjiza, DRJ Owen, and N Bicanic. "A combined finite-discrete element method in transient dynamics of fracturing solids". In: Engineering computations 12.2 (1995), pp. 145-174. 
[20] Eric Perkins and John R Williams. "Generalized spatial binning of bodies of different sizes". In: Discrete element methods: Numerical modeling of discontinua. 2002, pp. 52-55.

[21] E Rougier, A Munjiza, and NWM John. "Numerical comparison of some explicit time integration schemes used in DEM, FEM/DEM and molecular dynamics". In: International journal for numerical methods in engineering 61.6 (2004), pp. 856-879.

[22] John R Williams, Eric Perkins, and Ben Cook. "A contact algorithm for partitioning N arbitrary sized objects". In: Engineering Computations 21.2/3/4 (2004), pp. 235-248.

[23] Andrews Munjiza and KRF Andrews. "NBS contact detection algorithm for bodies of similar size". In: International Journal for Numerical Methods in Engineering 43.1 (1998), pp. 131-149.

[24] A Munjiza, DRJ Owen, and AJL Crook. "Energy and momentum preserving contact algorithm for general 2D and 3D contact problems." In: (2014).

[25] A Munjiza, KRF Andrews, and JR White. "Discretized contact solution for combined finitediscrete method". In: (2014).

[26] A Munjiza and KRF Andrews. "Penalty function method for combined finite-discrete element systems comprising large number of separate bodies". In: International Journal for Numerical Methods in Engineering 49.11 (2000), pp. 1377-1396.

[27] A Munjiza, T Bangash, and NWM John. "The combined finite-discrete element method for structural failure and collapse". In: Engineering Fracture Mechanics 71.4-6 (2004), pp. 469-483.

[28] Antonio A Munjiza. The combined finite-discrete element method. John Wiley \& Sons, 2004.

[29] Antonio Munjiza, KRF Andrews, and JK White. "Combined single and smeared crack model in combined finite-discrete element analysis". In: International Journal for Numerical Methods in Engineering 44.1 (1999), pp. $41-57$. 
[30] A Munjiza and KRF Andrews. "Penalty function method for combined finite-discrete element systems comprising large number of separate bodies". In: International Journal for Numerical Methods in Engineering 49.11 (2000), pp. 1377-1396.

[31] Antonio A Munjiza, Earl E Knight, and Esteban Rougier. Computational mechanics of discontinua. John Wiley \& Sons, 2011.

[32] Xinzheng Lu et al. "Numerical simulation for the progressive collapse of concrete building due to earthquake". In: a a 2 (2008), p. 2.

[33] T Bangash and A Munjiza. "A computationally efficient beam element for FEM/DEM simulations of structural failure and collapse". In: Discrete Element Methods: Numerical Modeling of Discontinua. 2002, pp. 133-137. DOI: https ://doi .org/10.1061/40647(259) 24.

[34] Nikolina Zivaljic, Hrvoje Smoljanovic, and Zeljana Nikolic. "A combined finite-discrete element model for RC structures under dynamic loading". In: Engineering Computations 30.7 (Oct. 2013), pp. 982-1010. DOI: 10 . 1108/ec-03-2012-0066.

[35] Hrvoje Smoljanovi, Nikolina ivalji, and eljana Nikoli. "A combined finitediscrete element analysis of dry stone masonry structures". In: Engineering Structures 52 (July 2013), pp. 89-100. DOI: 10.1016/j .engstruct.2013. 02.010 .

[36] Ye Jihong and Qi Nian. "Combination of DEM/FEM for Progressive Collapse Simulation of Domes Under Earthquake Action". In: International Journal of Steel Structures 18.1 (2018), pp. 305-316.

[37] A. Munjiza et al. "Fracture and fragmentation of thin shells using the combined finite-discrete element method". In: International Journal for Numerical Methods in Engineering 95.6 (June 2013), pp. 478-498. DOI: 10.1002/nme. 4511 .

[38] Kimiro Meguro and Motohiko Hakuno. "Fracture analyses of concrete structures by the modified distinct element method". In: Doboku Gakkai Ronbunshu 1989.410 (1989), pp. 113-124. 
[39] Motohiko Hakuno and Kimiro Meguro. "Simulation of concrete-frame collapse due to dynamic loading". In: Journal of engineering mechanics 119.9 (1993), pp. 1709-1723.

[40] Kimiro Meguro and Motohiko Hakuno. "Application of the extended distinct element method for collapse simulation of a doubledeck bridge". In: Doboku Gakkai Ronbunshu 1994.483 (1994), pp. 17-27.

[41] Limin Sun et al. "Application of extended distinct element method with lattice model to collapse analysis of RC bridges". In: Earthquake engineering \& structural dynamics 32.8 (2003), pp. 1217-1236.

[42] Atsushi ITOH, Junichiro NIWA, and Tada-aki TANABE. "Non-linear dynamic analysis of reinforced concrete piers based on lattice model". In: Doboku Gakkai Ronbunshu 2001.676 (2001), pp. 27-39.

[43] Tadahiko Kawai. "New element models in discrete structural analysis". In: Journal of the Society of Naval Architects of Japan 1977.141 (1977), pp. $174-180$.

[44] T Kawai. "Discrete Limit Analysis of Reinforced Concrete Structures Using Rigid Bodies-Spring Models". In: The finite element method in the 1990s. Springer, 1991, pp. 182-191.

[45] Tadahiko Kawai. "Some consideration on the finite element method". In: International Journal for Numerical Methods in Engineering 16.1 (1980), pp. $81-120$.

[46] Tadahiko Kawai and Yutaka Toi. "A discrete method of limit analysis and its application to plastic stability problems of structural members". In: Engineering Structures 5.1 (1983), pp. 38-44.

[47] Yutaka Toi. "Shifted integration technique in one-dimensional plastic collapse analysis using linear and cubic finite elements". In: International Journal for Numerical Methods in Engineering 31.8 (1991), pp. 1537-1552.

[48] E A. Tingatinga, H Kawakami, and S M. Shrestha. "Three-Dimensional Seismic Collapse Analysis of Wooden Houses Using Rigid Body-Spring Method". In: 2 (Sept. 2008), pp. 116-123. 
[49] Yutaka Toi and Daigoro Isobe. "Adaptively shifted integration technique for finite element collapse analysis of framed structures". In: International Journal for Numerical Methods in Engineering 36.14 (1993), pp. 23232339.

[50] Daigoro Isobe. Progressive Collapse Analysis of Structures: Numerical Codes and Applications. Butterworth-Heinemann, 2017.

[51] N Katahira et al. "Development of macro-model seismic collapse simulator for framed structures using ASI-Gauss technique". In: Proceedings of 14th WCEE (CD). Beijing: The 14th WVEE Organizing Committee (2008).

[52] M. Papadrakakis et al. A Dynamic Finite Element Code for Analyzing Collapse Behaviours of Framed Structures Under Seismic Loads.

[53] Kimiro Meguro and Hatem Tagel-Din. "A new efficient technique for fracture analysis of structures". In: Bulletin of Earthquake Resistant Structure Research Center, IIS, Univ. of Tokyo 30 (1997), pp. 103-116.

[54] Kimiro Meguro and Hatem Tagel-Din. "A new simplified and efficient technique for fracture behavior analysis of concrete structures". In: Proceedings of the Third International Conference on Fracture Mechanics of Concrete and Concrete Structures (FRAMCOS-3). Vol. 2. 1998, pp. 911920.

[55] Huda Helmy, Hamed Salem, and Sherif Mourad. "Computer-Aided Assessment of Progressive Collapse of Reinforced Concrete Structures according to GSA Code". In: Journal of Performance of Constructed Facilities 27.5 (Oct. 2013), pp. 529-539. DOI: 10.1061/(asce) cf.1943-5509.0000350.

[56] Nabil A. Rahman, Ayman Elfouly, and Michael Booth. "Alternate Path Progressive Collapse Analysis of Steel Stud Bearing Wall Structures". In: Structures Congress 2011. American Society of Civil Engineers, Apr. 2011. DOI: $10.1061 / 41171(401) 247$.

[57] Marin Lupoae and Carmen Bucur. "Building demolition - positive aspect of progressive collapse". In: 4 (Jan. 2009), p. 10. 
[58] Said Elkholy, Hatem Tagel-Din, and Kimiro Meguro. "Structural failure simulation due to fire by applied element method". In: JCOSSAR2003, the Fifth Japan Conference on Structural Safety and Reliability, Tokyo, Japan. 2003.

[59] K Worakanchana and K Meguro. "Voronoi Applied Element Method For Structural Analysis: Theory And Application For Linear And Non-Linear Materials". In: The 14th World Conference on Earthquake Engineering October. 2008, pp. 12-17.

[60] Nam-Ho Kim. Introduction to nonlinear finite element analysis. Springer Science \& Business Media, 2014.

[61] Junuthula Narasimha Reddy. Theory and analysis of elastic plates and shells. CRC press, 2006.

[62] Detailed Explanation of the Finite Element Method (FEM). Sept. 10, 2018. URL: https://www.comsol . com/multiphysics/finite-elementmethod (visited on 09/10/2018).

[63] Antonio Munjiza, Esteban Rougier, and Earl Knight. Large Strain Finite Element Method: A Practical Course. Nov. 2014. ISBN: 978-1-118-40530-7.

[64] K Liu and W Liu. "Application of discrete element method for continuum dynamic problems". In: Archive of Applied Mechanics 76.3-4 (2006), pp. 229-243.

[65] ă ăPrashidha Kharel's Blog: Formulating the Applied Element Method: Linear 2D (Part I). Aug. 29, 2018. URL: http://blog.prashidha.com/ 2014 / 03 / formulating - applied-element-method . html (visited on 09/05/2018).

[66] Kimiro Meguro and H Sayed Tagel-Din. "Applied element method used for large displacement structural analysis". In: Journal of Natural Disaster Science 24.1 (2002), pp. 25-34.

[67] A Okabe et al. Spatial tesselations: concepts and applications of Voronoi tesselations. 2000. 
[68] Eduardo A de Souza Neto, Djordje Peric, and David RJ Owen. Computational methods for plasticity: theory and applications. John Wiley \& Sons, 2011.

[69] Kenneth Runesson et al. "Constitutive modeling of engineering materialstheory and computation". In: Volume I General Concepts and Inelasticity (2006).

[70] JC Simo and TJR Hughes. Computational Inelasticity, Interdisciplinary Applied Mathematics 7. 1998.

[71] Louie L Yaw. "Nonlinear static1D plasticityvarious forms of isotropic hardening". In: Walla Walla University 25 (2012).

[72] Dr Ulrich Hoppe. "Computational Plasticity". In: Lecture Notes,. Ruhr University Bochum (2010).

[73] Djordje Peric. "Computational Plasticity". In: Lecture Notes, Swansea University (2013).

[74] Rade Vignjevic et al. "Modelling of strain softening materials based on equivalent damage force". In: Computer Methods in Applied Mechanics and Engineering 335 (June 2018), pp. 52-68. DOI: 10.1016/j.cma.2018. 01.049 .

[75] Zdenk P. Baant, Ted B. Belytschko, and Ta-Peng Chang. "Continuum Theory for Strain-Softening". In: Journal of Engineering Mechanics 110.12 (Dec. 1984), pp. 1666-1692. DOI: 10.1061/(asce)0733-9399(1984)110: $12(1666)$.

[76] Rodney Hill. "Acceleration waves in solids". In: Journal of the Mechanics and Physics of Solids 10.1 (1962), pp. 1-16.

[77] Jean Mandel. "Conditions de stabilité et postulat de Drucker". In: Rheology and Soil Mechanics/Rhéologie et Mécanique des Sols. Springer, 1966, pp. $58-68$. 
[78] AH Marchertas, RF Kulak, and YC Pan. "Performance of blunt crack approach within a general purpose code". In: Nonlinear Numerical Analysis of Reinforced Concrete, ed., LE Schwer, Am. Soc. of Mech. Engrs., New York (1982), pp. 107-123.

[79] Clifford Truesdell and Walter Noll. "The non-linear field theories of mechanics". In: The non-linear field theories of mechanics. Springer, 2004, pp. $1-579$.

[80] Jacques Hadamard. Lecons Sur Un Propogation Des Ondes. 1903.

[81] Anil K Chopra. Dynamics of structures: Theory and applications. 2001.

[82] Nathan M Newmark. "A method of computation for structural dynamics". In: Journal of the engineering mechanics division 85.3 (1959), pp. 67-94.

[83] Henri Gavin. "Numerical integration for structural dynamics". In: ().

[84] Georg GroSSeholz, Delfim Soares, and Otto Estorff. "A stabilized central difference scheme for dynamic analysis". In: International Journal for Numerical Methods in Engineering 102.11 (2015), pp. 1750-1760.

[85] Mass Matrix Construction Overview. Online. URL: https: //www . colorado. edu / engineering/CAS / courses . d/MFEMD . d/MFEMD . Ch16 . d /MFEMD . Ch16.pdf.

[86] Ronald Hamburger and Andrew Whittaker. "Design of steel structures for blast-related progressive collapse resistance". In: 44 (Mar. 2004), pp. 4153.

[87] Roberto Villaverde. "Methods to Assess the Seismic Collapse Capacity of Building Structures: State of the Art". In: Journal of Structural Engineering 133.1 (Jan. 2007), pp. 57-66. DOI: 10.1061/(asce)0733-9445(2007) $133: 1(57)$.

[88] Earthquake Engineering Research Institute. Learning from Earthquakes Program, Loring A Wyllie, and Bruce A Bolt. The chile earthquake of March 3, 1985. The Institute, 1986. 
[89] Roberto Villa Verde. "Explanation for the numerous upper floor collapses during the 1985 Mexico city earthquake". In: Earthquake Engineering \& Structural Dynamics 20.3 (1991), pp. 223-241. DOI: 10 . 1002 / eqe . 4290200303.

[90] J. Osteraas and H. Krawinkler. "The Mexico Earthquake of September 19, 1985Behavior of Steel Buildings". In: Earthquake Spectra 5.1 (Feb. 1989), pp. 51-88. DOI: 10.1193/1.1585511.

[91] Anshel J Schiff. "Earthquake spectra. Philippines Earthquake reconnaissance report". In: Earthquake spectra 7 (1991), pp. 1-141.

[92] Craig D Comartin. Guam earthquake of August 8, 1993. Earthquake Engineering Research Institute, 1993.

[93] John F Hall, WT Holmes, and P Somers. "Northridge earthquake, January 17, 1994". In: Preliminary reconnaissance report (1994).

[94] Alex K Tang. Izmit (Kocaeli), Turkey, Earthquake of August 17, 1999 Including Duzce Earthquake of November 12, 1999: Lifeline Performance. Vol. 17. ASCE Publications, 2000.

[95] Joseph Uzarski and Christopher Arnold. Chi-Chi, Taiwan, earthquake of September 21, 1999: reconnaissance report. Vol. 17. Earthquake Engineering Research Institute, 2001.

[96] JV Hengesh et al. "Bhuj, india earthquake of january 26, 2001-reconnaissance report". In: (2002).

[97] Deadliest Earthquakes in History. July 23, 2018. URL: https : / / www . livescience.com/6932-deadliest-earthquakes-history.html (visited on $07 / 23 / 2018)$.

[98] Seth Stein and Michael Wysession. An introduction to seismology, earthquakes, and earth structure. John Wiley \& Sons, 2009.

[99] BBC - GCSE Bitesize: Causes. Aug. 10, 2015. URL: http://www . bbc.co. uk/schools/gcsebitesize/geography/natural_hazards/earthquakes_ rev1.shtml (visited on 07/23/2018). 
[100] Michael Wysession Seth Stein. An Introduction to Seismology, Earthquakes and Earth Structure. John Wiley and Sons Ltd, Sept. 1, 2002. 512 pp. ISBN: 0865420785. URL: https : //www. ebook.de/de/product/ $3770366 /$ seth_stein_michael_wysession_an_introduction _ to _ seismology_earthquakes_and_earth_structure.html.

[101] Sumatra, Indonesia, Dec. 26, 2004. July 23, 2018. URL: http://geosurvey . ohiodnr.gov/beyond-border-region-quake-events/sumatra-indonesiadec-26-2004 (visited on 07/23/2018).

[102] Harry O Wood and Frank Neumann. "Modified Mercalli intensity scale of 1931". In: Bulletin of the Seismological Society of America 21.4 (1931), pp. $277-283$.

[103] Kobe Earthquake. Feb. 16, 2012. URL: http://www.vibrationdata.com/ earthquakes/kobe.htm (visited on 07/23/2018).

[104] The Editors of Encyclopaedia Britannica, ed. Kbe earthquake of 1995. Encyclopædia Britannica, inc., Apr. 2017. URL: https://www. britannica. com/event/Kobe-earthquake-of-1995.

[105] Dr. Amit Srivastava, Chaitanya Goyal, and Akash Jain. Review of Causes of foundation failures and their possible preventive and remedial measures. Jan. 2012.

[106] Structural engineering aspects of the June 27, 1998 AdanaCeyhan (Turkey) earthquake - ScienceDirect. July 24, 2018. URL: https: //www . sciencedirect. com/science/article/pii/S0141029600000468 (visited on 07/24/2018).

[107] January 17 in the Ring of Fire | Multimedia | teleSUR English. July 23, 2018. URL: https://www.telesurtv .net/english/multimedia/January17-in-the-Ring-of-Fire-20150117-0022.html (visited on 07/23/2018).

[108] Alamy Limited. A satellite image of Sumatra, Indonesia, before and after the Stock Photo: 103996337 - Alamy. July 23, 2018. URL: https : //www . alamy . com/stock-photo-a-satellite-image-of-sumatraindonesia-before-and-after-the-devasting-103996337 .html (visited on $07 / 23 / 2018)$. 
[109] Jacob C. Earthquake Database. Feb. 16, 2017. URL: http://web.iitd. ac. in/ matsagar/EqDataBase.htm (visited on 07/24/2018).

[110] Svend Ole Hansen Claes Dyrbye. Wind Loads on Structures. John Wiley \& Sons, 1997. ISBN: 0471956511.

[111] Lorenzo Rosa et al. "Wind-induced dynamics and loads in a prismatic slender building: A modal approach based on unsteady pressure measurements". In: 107-108 (Aug. 2012), pp. 118-130.

[112] Munich Reinsurance. Topics Geo annual review: Natural catastrophes 2004. 2004 .

[113] Yukio Tamura. "Wind-induced damage to buildings and disaster risk reduction". In: Proceedings of the APCWE-VII, Taipei, Taiwan (2009).

[114] Kangpyo Cho et al. "Typhoon (Maemi) attack and its damage in Southern Korea celebrating Thanksgiving festival of Chusok". In: Sixth Asia-Pacific Conference on Wind Engineering. 2005.

[115] Edifício Real Class - Construtora Real. Aug. 14, 2018. uRL: http : // compradordeimovel . no . comunidades . net/edificio-real-classconstrutora-real (visited on 08/14/2018).

[116] Sigrid Reiter. "Validation process for CFD simulations of wind around buildings". In: Proceedings of the European Built Environment CAE Conference. Londres. 2008, p. 18.

[117] Shuzo Murakami. "Overview of turbulence models applied in CWE--1997". In: Journal of Wind Engineering and Industrial Aerodynamics 74 (1998), pp. 1-24.

[118] BG Wiren. "A wind tunnel study of wind velocities in passages between and through buildings". In: Proceedings of the 4th International Conference on Wind Effects on Buildings and Structures,(Heathrow 1975). 1975, pp. $465-475$.

[119] Aly Mousaad Aly. "The role of turbulence on the response of buildings to extreme wind". In: (). 